\title{
Rauhigkeit und Durchmischung der Grenzflächen in laserdeponierten Cu-Ag- und Fe-Ag-Schichtsystemen
}

\author{
Dissertation \\ zur Erlangung des Doktorgrades \\ der Mathematisch-Naturwissenschaftlichen Fakultäten \\ der Georg-August-Universität zu Göttingen
}

\author{
vorgelegt von \\ Martin Weisheit \\ aus Erfurt
}

Göttingen 2002 
D 7

Referent:

Prof. Dr. H.-U. Krebs

Korreferent:

Prof. Dr. R. G. Ulbrich

Tag der mündlichen Prüfung: $\quad$ 25.10.2002 


\section{Inhalt}

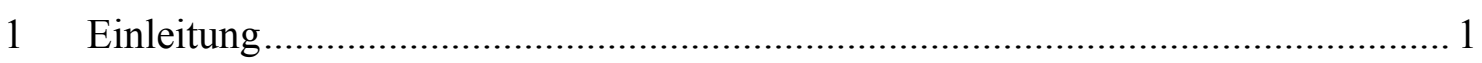

2 Aufbau, Meß- und Auswerteverfahren ............................................................ 5

2.1 Gepulste Laserdeposition............................................................................ 5

2.2 Bestimmung von Depositionsrate und Schichtdicke ....................................... 6

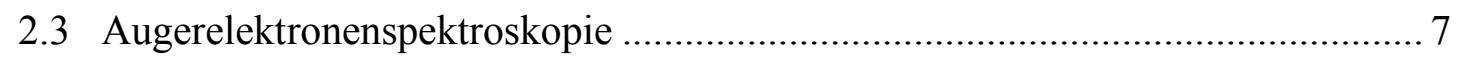

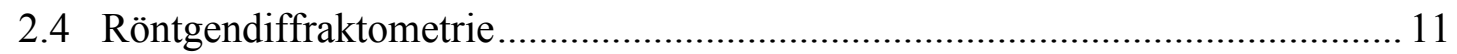

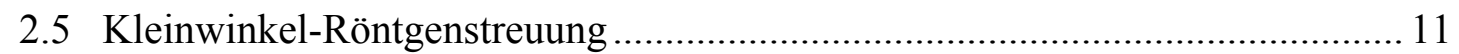

2.6 Konversionselektronen-Mößbauerspektroskopie ........................................... 18

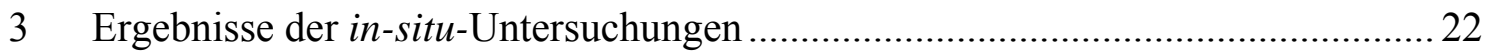

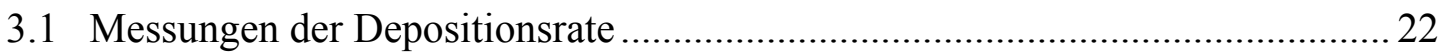

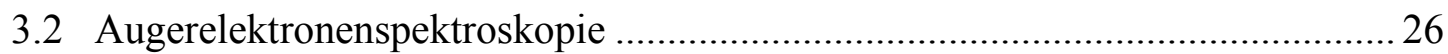

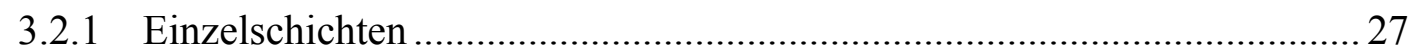

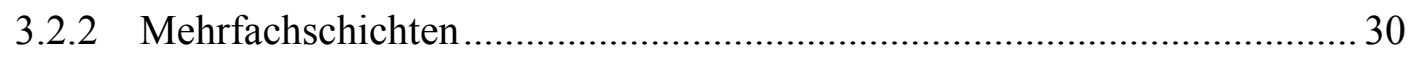

3.3 Modelle zum Verlauf der Oberflächenkonzentration mit der Schichtdicke ....... 34

3.3.1 Oberflächenkonzentration bei glatten Grenzflächen ................................ 34

3.3.2 Numerisches Modell des Schichtwachstums bei der PLD ......................... 36

3.3.3 Simulation der Oberflächenkonzentration ............................................ 41

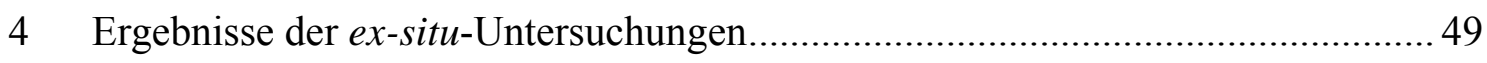

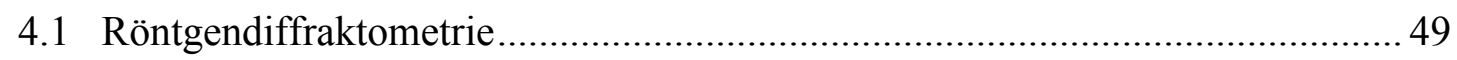

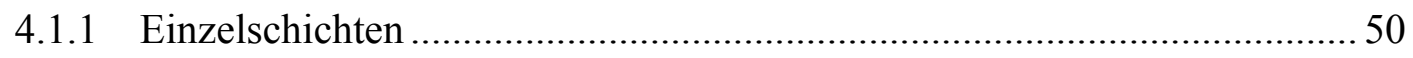

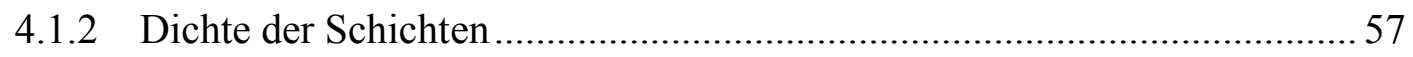

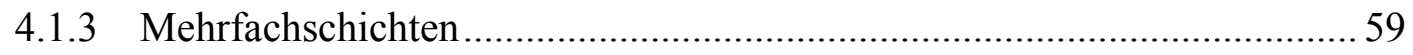

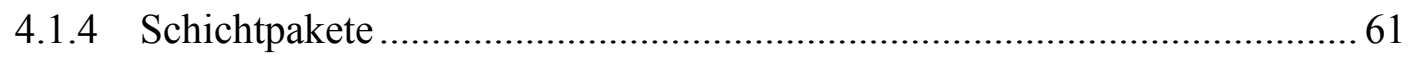

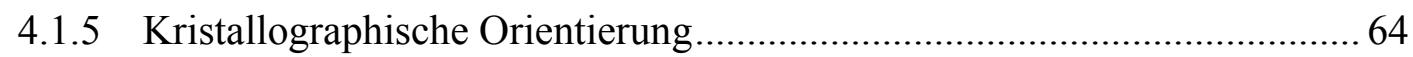


4.2 Mößbauerspektroskopie

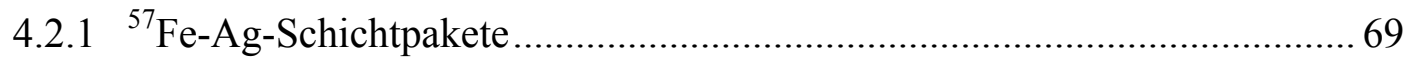

4.2.2 Fe-Ag-Schichtpakete mit ${ }^{57}$ Fe-Markerschichten .................................. 73

4.2.3 Ratenabhängigkeit der Grenzflächendurchmischung ............................. 77

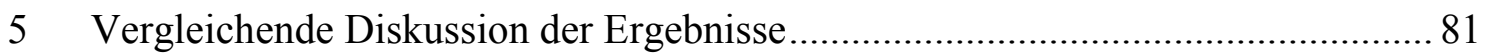

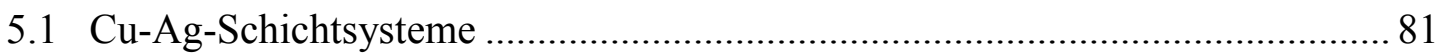

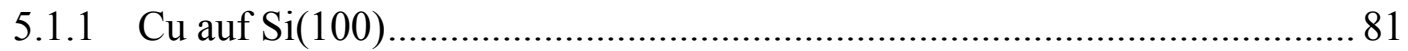

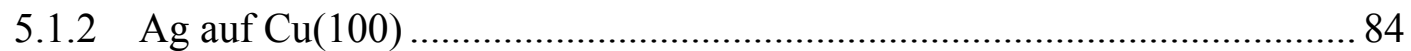

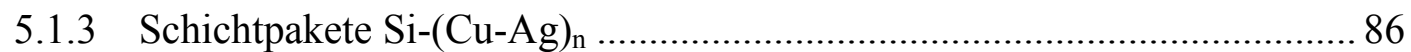

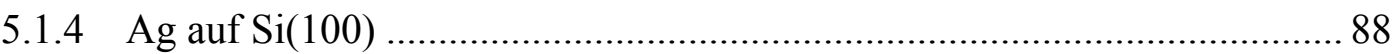

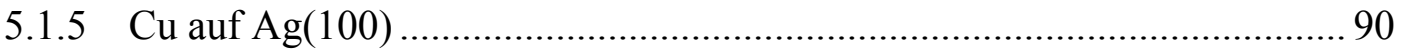

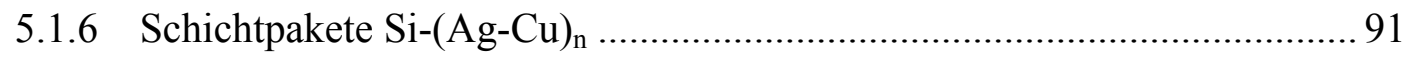

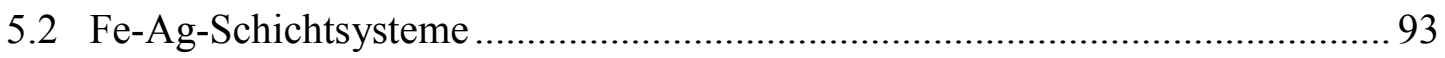

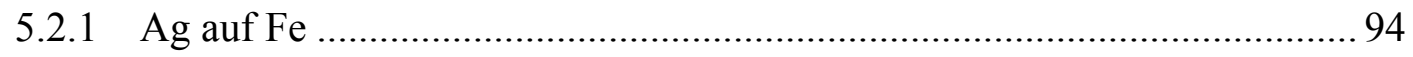

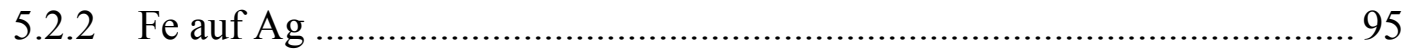

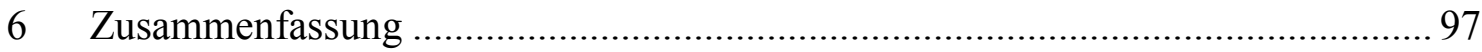

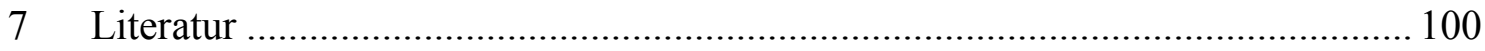




\section{Einleitung}

Die Eigenschaften dünner Schichten auf Si sind ein seit langem intensiv untersuchtes Forschungsgebiet, was in den vielseitigen Anwendungsmöglichkeiten insbesondere in der Mikroelektronik begründet liegt. Mit zunehmender Miniaturisierung beeinflussen immer mehr die Grenzflächen zwischen den Komponenten die Eigenschaften des Gesamtsystems, weshalb das Interesse an der Struktur und Zusammensetzung von Grenzflächen stetig wächst. Beispielsweise wird seit einigen Jahren versucht, in hochintegrierten Schaltkreisen die aus Al bestehenden Leiterbahnen durch solche aus $\mathrm{Cu}$ [1] und möglicherweise $\mathrm{Ag}$ [2] $\mathrm{zu}$ ersetzen. Neben $\mathrm{Ag}$ besitzt $\mathrm{Cu}$ die beste elektrische Leitfähigkeit aller Elemente und ist nicht so anfällig für Elektromigration wie Al [3], diffundiert allerdings bei erhöhten Temperaturen (wie sie schon durch die thermische Belastung beim Betrieb von Prozessoren auftreten) in das Si-Substrat hinein und bildet eine breiter werdende $\mathrm{Cu}_{3} \mathrm{Si}$-Grenzfläche aus [4], was letztendlich zur Zerstörung der Leiterbahn führt. Die zuverlässige Unterbindung der Interdiffusion und der Ausbildung einer breiten Grenzfläche mittels Diffusionsbarrieren aus anderen Materialien $[5,6]$ ist gegenwärtig ein wichtiges Ziel in der Halbleiterindustrie.

Neben den üblicherweise für die Dünnschichtpräparation eingesetzten Methoden Sputtern und Aufdampfen hat sich die gepulste Laserdeposition (Pulsed Laser Deposition - PLD), die vor etwa 15 Jahren als Methode der Wahl für die Deposition von Hochtemperatursupraleitern eingeführt wurde, auch für andere Materialien als flexible Depositionsmethode etabliert. Ihre besonderen Eigenschaften im Vergleich zu den traditionellen Beschichtungsverfahren und ihre Anwendbarkeit auf die verschiedensten Materialklassen werden seitdem intensiv untersucht. Einen Überblick über das Gebiet liefert z. B. [7] und im Fall der metallischen Systeme [8]. Insbesondere lassen die bei der Laserdeposition auftretenden Ionen mit Energien um $100 \mathrm{eV}$ neben dem teilweisen Wiederabtrag schon deponierten Materials [9,10] eine Implantation in die aufwachsende Schicht und damit eine Durchmischung der Grenzflächen erwarten $[11,12,13]$.

Ziel dieser Arbeit ist es, den Aufbau der Grenzflächen in laserdeponierten Cu-Ag- und Fe-Ag-Schichtsystemen detailliert aufzuklären und dabei insbesondere den Einfluß der energiereichen Ionen zu untersuchen. Dazu kommen eine Reihe von komplementären, sich ergänzenden Untersuchungsmethoden zum Einsatz: in-situ wird die Oberfläche der 
Proben mittels Augerelektronenspektroskopie und Ratenmessungen untersucht, ex-situ werden Kleinwinkel-Röntgenstreuung und im Fall der Fe-Ag-Schichtsysteme Konversionselektronen-Mößbauerspektroskopie eingesetzt. Durch den Vergleich der Ergebnisse mit Simulationsrechnungen der Röntgenstreuung in Schichtpaketen sowie der Ionenimplantation bzw. des Sputterns der Oberfläche bei der Schichtherstellung sollen quantitative Aussagen zu Grenzflächenrauhigkeit und -durchmischung sowie zum Grenzflächenprofil in diesen Systemen gewonnen werden.

Als Modellsystem wurde $\mathrm{Cu}-\mathrm{Ag}$ gewählt, weil einerseits beide Elemente die gleiche Kristallstruktur (kubisch flächenzentriert - fcc) besitzen und andererseits keine Tendenz für eine thermodynamisch getriebene Durchmischung der Grenzflächen existiert, da die Mischungsenthalpie des Systems positiv ist. Dies erlaubt die Untersuchung der rein ballistischen Mischung durch die schnellen Ionen. Mit 4,8 kJ/mol [14] ist die Mischungsenthalpie dabei klein genug, daß $\mathrm{Cu}$-Ag-Legierungen beliebiger Konzentration bei Raumtemperatur nicht entmischen [15]. Einmal ausgebildete durchmischte Grenzflächen bleiben somit stabil.

Das Fe-Ag-System unterscheidet sich vom System $\mathrm{Cu}-\mathrm{Ag}$ durch seine hohe positive Mischungsenthalpie von $28 \mathrm{~kJ} / \mathrm{mol}$ [16], welche eine Entmischung während der Deposition bei Raumtemperatur bewirken kann. Im Gegensatz zu Ag besitzt Fe eine kubisch raumzentrierte Kristallstruktur (bcc), was einen komplizierten Wachstumsmodus (Volumentransformation zwischen fcc und bcc) der beiden Elemente aufeinander zur Folge hat [17]. Außerdem kommt es bei der Laserdeposition von Fe auf Ag zu einem teilweisen Absputtern der Ag-Schicht, was möglicherweise den Aufbau der Grenzfläche beeinflußt. Die Fe-Ag-Grenzfläche soll unter Berücksichtigung dieser Besonderheiten charakterisiert werden.

Das Interesse am Aufbau der Fe-Ag-Grenzfläche rührt daher, daß Ag oft als Substratschicht für darauf deponierte Fe-Schichtsysteme dient [18], welche aufgrund ihrer besonderen magnetischen Eigenschaften, wie z. B. dem GMR-Effekt (Riesenmagnetowiderstand), untersucht werden [19,20]. Rauhigkeit und Durchmischung der Grenzfläche haben dabei einen entscheidenden (i. a. negativen) Einfluß auf diese Effekte.

Nach einer Einführung in die verwendeten Untersuchungsmethoden und der theoretischen Darstellung der zugrundeliegenden Mechanismen in Kap. 2 werden in dieser Arbeit zunächst die Ergebnisse der in-situ durchgeführten Messungen dargestellt. 
Eingesetzt wurden hier neben der Augerelektronenspektroskopie (Kap. 3.2) Messungen der Depositionsrate (Kap. 3.1). Allein aus den Ratenmessungen während der Herstellung lassen sich bereits Aussagen über den Verlauf der Oberflächenkonzentration mit der Schichtdicke gewinnen, wenn die Messungen im Rahmen eines Modells des Sputtervorganges [9] an der Oberfläche der wachsenden Schicht interpretiert und ausgewertet werden. Für eine direktere und genauere Messung der Oberflächenkonzentration wurde ein System zur Augerelektronenspektroskopie aufgebaut und in die Steuerung der vorhandenen PLD-Anlage integriert. Ziel ist es, während der Deposition der Schichten in regelmäßigen Abständen automatisch Spektren aufzunehmen und daraus die Oberflächenkonzentration des deponierten Elementes als Funktion der deponierten Schichtdicke zu bestimmen. Mit Hilfe eines numerischen Modells des Wachstums laserdeponierter Filme, welches das aus der bekannten Geschwindigkeitsverteilung der Ionen berechnete Implantationsprofil berücksichtigt, kann der Verlauf der Oberflächenkonzentration simuliert und mit der Messung verglichen werden. Auf diese Weise wird über die simulierten Schichtpakete auch der Konzentrationsverlauf in den inneren Grenzflächen zugänglich.

Im darauf folgenden Kapitel werden die Ergebnisse der ex-situ-Untersuchungen vorgestellt. Die Kleinwinkel-Röntgenreflektometrie (Kap. 4.1.1 bis 4.1.4) erlaubt über den Vergleich der gemessenen Diffraktogramme mit einer Simulation der Röntgenstreuung in Mehrschichtsystemen eine quantitative Bestimmung von Oberflächenrauhigkeiten und Grenzflächenbreiten mit atomarer Auflösung. Um unterscheiden zu können, ob es sich bei der Breite der inneren Grenzflächen um eine Grenzflächenrauhigkeit oder -durchmischung handelt, werden im Fall des $\mathrm{Cu}-\mathrm{Ag}$ Systems zunächst Einzelschichten verschiedener Dicke auf ihre Oberflächenrauhigkeiten hin untersucht. Im Vergleich mit Doppelschichten kann dann auf den Einfluß von Durchmischung und Rauhigkeit auf die Grenzflächenbreite geschlossen werden. Der Einfluß des Wachstumsmodus und eventueller Epitaxiebeziehungen zwischen $\mathrm{Cu}$ und $\mathrm{Ag}$ wird in diesem Zusammenhang mittels Röntgendiffraktometrie im Großwinkelbereich und Texturuntersuchungen bestimmt (Kap. 4.1.5).

Für die ex-situ-Untersuchung der Fe-Ag-Schichten kam hauptsächlich die Konversionselektronen-Mößbauerspektroskopie zum Einsatz (Kap. 4.2), da mit dem ${ }^{57} \mathrm{Fe}$-Isotop eine empfindliche Sonde für die Fremdatomkonzentration in den FeSchichten zur Verfügung steht. Dazu wurden neben reinen ${ }^{57} \mathrm{Fe}-\mathrm{Ag}$-Schichtpaketen 
auch Fe-Ag-Schichtpakete mit ${ }^{57} \mathrm{Fe}-\mathrm{Markerschichten}$ in verschiedenen Abständen von der Fe-Ag-Grenzfläche untersucht, um die Ag-Konzentration als Funktion des Abstandes zur Grenzfläche bestimmen zu können. Da aufgrund der stark positiven Mischungsenthalpie im System Fe-Ag mit einer Entmischung der Grenzflächen schon während der Deposition gerechnet werden muß, wird die Stärke der Durchmischung auch in Abhängigkeit von der Depositionsrate untersucht, um deren möglichen Einfluß auf die Entmischungskinetik zu bestimmen.

In der vergleichenden Diskussion (Kap. 5) werden die Ergebnisse der in-situ- und exsitu-Methoden in einem gemeinsamen Rahmen verglichen und ein Modell zum Wachstum laserdeponierter $\mathrm{Cu}-\mathrm{Ag}$ - und $\mathrm{Fe}-\mathrm{Ag}$-Schichten sowie dem Aufbau der Grenzflächen vorgestellt, das konsistent mit den Beobachtungen ist.

Abschließend werden die wichtigsten Ergebnisse und Hauptaussagen der Arbeit noch einmal zusammengefaßt (Kap. 6). 


\section{Aufbau, Meß- und Auswerteverfahren}

\subsection{Gepulste Laserdeposition}

Die in dieser Arbeit untersuchten Schichten wurden mit dem Verfahren der gepulsten Laserdeposition hergestellt, das schematisch in Abbildung 2-1 skizziert ist. Dabei werden die energiereichen, $30 \mathrm{~ns}$ langen Laserpulse eines KrF-Excimerlasers (Lambda Physik LPX 110i, Wellenlänge $248 \mathrm{~nm}$ ) auf ein Metalltarget in einer UHV-Kammer mit einem Basisdruck von $10^{-9}$ mbar fokussiert. Auf der bestrahlten Fläche von $2 \mathrm{~mm}^{2}$ werden bei Laserpulsenergien zwischen 60 und $160 \mathrm{~mJ}$ Energiedichten von 3 bis $8 \mathrm{~J} / \mathrm{cm}^{2}$ erreicht. Die entsprechend hohen Leistungsdichten von einigen $10^{8} \mathrm{~W} / \mathrm{cm}^{2}$ führen zu einer schnellen Temperaturerhöhung in einer dünnen Oberflächenschicht des Targets. Die Oberfläche verdampft und bildet ein dichtes Plasma aus, das mit hoher Geschwindigkeit senkrecht zur Targetoberfläche expandiert [21]. Dieses Plasma trifft je nach Stellung des Targets nach einer Flugstrecke von einigen Zentimetern entweder auf einem Si-Substrat (Substratposition 1 in Abbildung 2-1) oder einem Ratenmonitor (Substratposition 2) auf. Diese beiden Substratpositionen sind unter $45^{\circ}$ symmetrisch um den einfallenden Laserstrahl gruppiert, so daß durch eine Drehung des Targets in

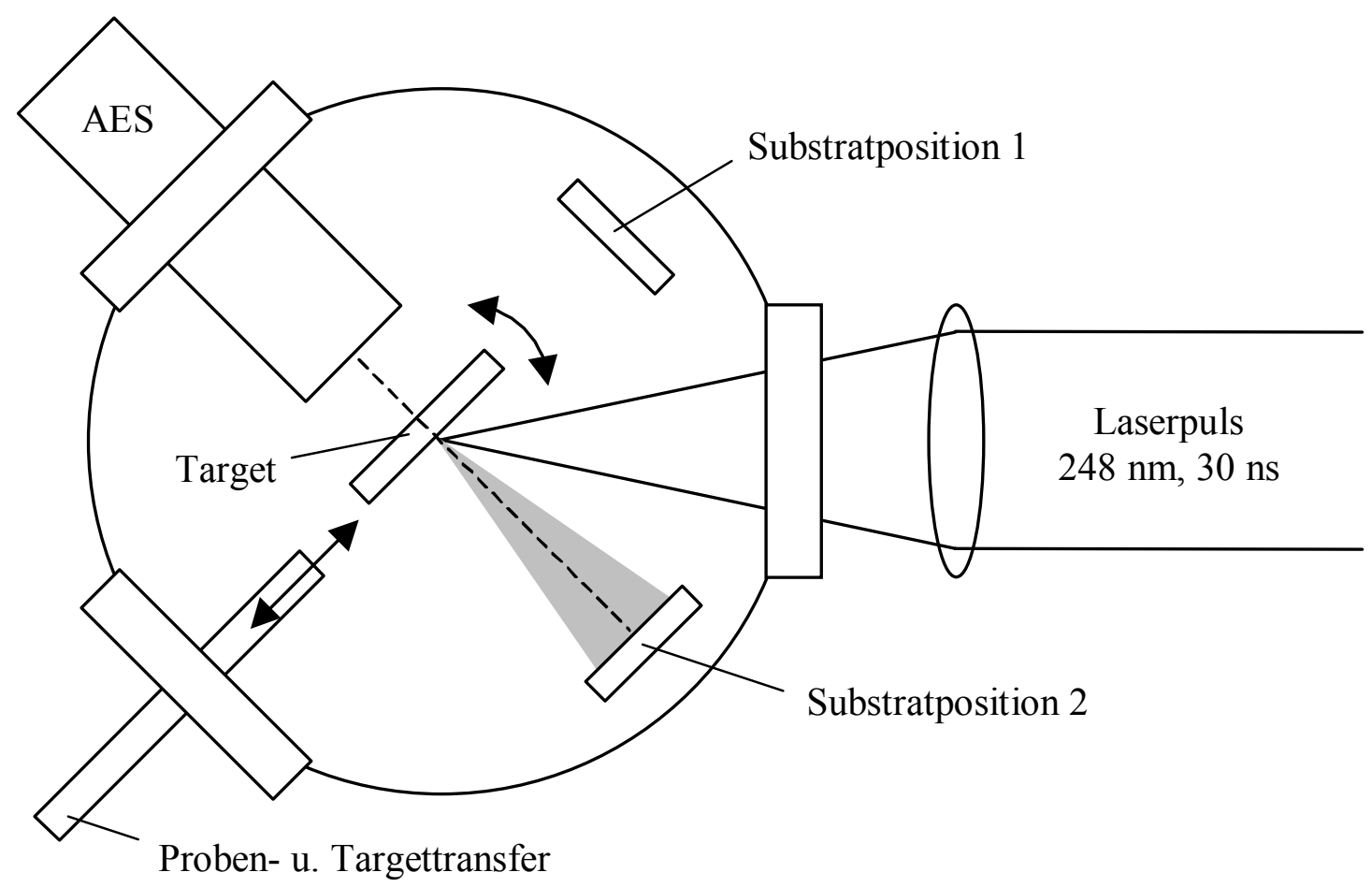


Richtung des Ratenmonitors die Depositionsrate ständig kontrolliert werden kann. Die Entfernung zwischen Target und Substratposition 1 kann variiert werden, wurde aber (mit Ausnahme der in Kap. 4.2.3 beschriebenen Untersuchungen) für alle Experimente in dieser Arbeit auf den maximalen Abstand von $68 \mathrm{~mm}$ festgelegt, um eine möglichst hohe Schichthomogenität zu gewährleisten. Dem gleichen Ziel dient die zusätzliche Drehung des Substrates während der Deposition.

Substrate an der Substratposition 1 und die Targets können über ein Schleusensystem in die Kammer eingeführt und wieder entnommen werden, ohne das Vakuum zu brechen. Für die Schleuse wurde ein kombiniertes Substrat-/Targetmagazin konstruiert, das maximal 28 Substrate bzw. Targets aufnehmen kann. Damit ist es möglich, viele Schichten aus unterschiedlichsten Materialien hintereinander unter gleichen Bedingungen zu deponieren.

\subsection{Bestimmung von Depositionsrate und Schichtdicke}

Wie oben erwähnt, wird die Depositionsrate über einen Ratenmonitor (Leybold Inficon $\mathrm{XTM} / 2$ ) an Substratposition 2 kontrolliert, der auf dem Prinzip der Schwingquarzwaage basiert. Dabei wird ein Quarzkristallplättchen zu Eigenschwingungen angeregt, deren Frequenz mit der Masse des auf dem Kristall deponierten Materials abnimmt. Über die Messung dieser Eigenfrequenz und unter Berücksichtigung der Dichte des deponierten Materials läßt sich die Schichtdicke berechnen. Neben der Masse haben auch die mechanischen Eigenschaften des Materials einen geringen Einfluß auf die Schwingungsfrequenz. Deshalb können bei hohen Anforderungen an die Genauigkeit der Messung auch die elastischen Konstanten der deponierten Schicht in Form eines Korrekturfaktors berücksichtigt werden.

Der Ratenmonitor befindet sich nicht wie das Substrat in $68 \mathrm{~mm}$ Entfernung zum Target, sondern hat nur einen maximalen Abstand von $57 \mathrm{~mm}$, was eine Umrechnung der vom Ratenmonitor gemessenen Depositionsrate $R_{R M}$ auf die Rate am Ort des Substrats $R_{\text {Sub }}$ nötig macht. Geht man näherungsweise von einem punktförmigen Brennfleck des Laserpulses auf dem Target aus, sollte die Rate $R$ quadratisch mit der Entfernung $d$ abnehmen. In der Praxis ergibt sich nach [22] für die vorliegende Depositionsgeometrie ein Exponent von 2,25 und damit für das Verhältnis der Raten auf dem Substrat und auf dem Ratenmonitor bei den Abständen $d_{S u b}$ und $d_{R M}$ : 


$$
\frac{R_{S u b}}{R_{R M}}=\left(\frac{d_{S u b}}{d_{R M}}\right)^{-2,25}
$$

Nach der Herstellung der Schicht kann die Schichtdicke mittels eines Profilometers (Perthometer der Firma Perthen) überprüft werden. Dabei wird eine empfindliche Nadel über die Probe gefahren und ihre vertikale Auslenkung gemessen. Wurde zuvor während der Deposition ein Teil des Substrats abgedeckt, um eine Beschichtung an dieser Stelle zu verhindern, kann man die Höhe der auf diese Weise entstandenen Stufe direkt ausmessen und rückwirkend die Depositionsrate bestimmen. Die Auflösung des Gerätes liegt bei $10 \mathrm{~nm}$, was bei einer typischen Schichtdicke von $200 \mathrm{~nm}$ eine Unsicherheit von 5\% zur Folge hat. Eine genauere Bestimmung der Schichtdicke ist mit Hilfe der Kleinwinkel-Röntgenstreuung möglich (siehe Kap. 2.5).

Die in-situ-Kontrolle der Rate während der Deposition der Schichten ist wichtig, weil aufgrund der unvermeidlichen Beschichtung der Eintrittsfenster ein wachsender Teil der Laserpulsenergie das Target nicht erreicht und damit die Rate abnimmt. Bei der Präparation von Schichtpaketen mit vielen Einzelschichten führt das $\mathrm{zu}$ einer abnehmenden Einzelschichtdicke mit steigender Gesamtschichtdicke, was durch eine Nachregelung der Laserpulsenergie kompensiert werden muß.

\subsection{Augerelektronenspektroskopie}

In der UHV-Kammer für die Laserdeposition wurde auf der Linie TargetSubstratposition 2 hinter dem Target ein Augerelektronen-Spektrometer (Staib DESA 100) integriert. Das Target kann abgesenkt werden und gibt so das Substrat bzw. den Schwingquarz für die Analyse mittels AES frei (Abbildung 2-2). Bei der Verwendung des Schwingquarzes können gleichzeitig Dicke und Konzentration bestimmt werden. Der Arbeitsabstand des Spektrometers ist mit $55 \mathrm{~mm}$ groß genug, um eine Messung ohne Verschiebung des Spektrometers zum Substrat hin zu ermöglichen. Auf diese Weise können Augerspektren einerseits sehr schnell (wichtig, um Oberflächenkontamination $\mathrm{zu}$ vermeiden) und sehr gut reproduzierbar (weil der Arbeitsabstand konstant bleibt) aufgenommen werden. Durch eine Synchronisation der rechnergestützten Ansteuerung der Depositionsanlage und des AugerelektronenSpektrometers wurde es möglich, in regelmäßigen Abständen während der Schichtherstellung Augerspektren aufzunehmen. 


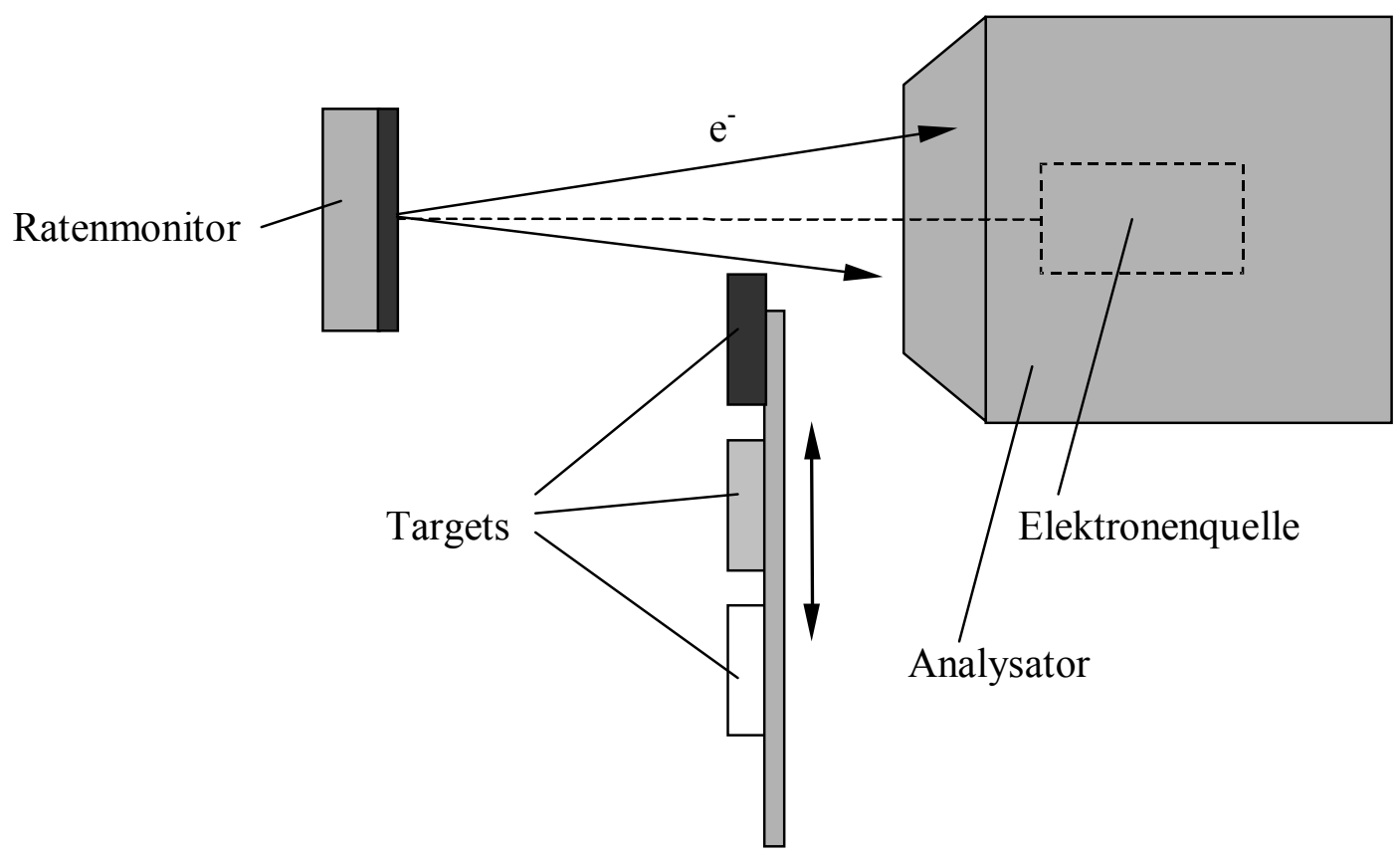

Abbildung 2-2: Aufbau der AES-Apparatur

Das zugrundeliegende Prinzip der Augerelektronenemission ist in Abbildung 2-3 dargestellt und ist z. B. in [23] ausführlich beschrieben. Hier sollen nur die für die folgenden Untersuchungen relevanten Aspekte dargestellt werden.

Die Probe wird mit einem Elektronenstrahl von $5 \mathrm{keV}$ angeregt, was zu einer Ionisation eines Teils der Probenatome führt. Wird durch eines der Primärstrahlelektronen ein Elektron aus einer energetisch tief liegenden Schale herausgeschlagen (1), füllt ein Elektron aus einem höheren Niveau die entstandene Lücke (2). Die Energiedifferenz kann entweder in Form eines Photons abgegeben werden, oder es wird ein weiteres Elektron emittiert (3). Bei schweren Elementen überwiegt die Photonenemission, dagegen ist die Wahrscheinlichkeit für die Emission eines Elektrons nahezu konstant [24]. Die kinetische Energie $E_{k i n}$ dieser Augerelektronen ist unabhängig von der Energie des anregenden Elektronenstrahls und charakteristisch für jedes Element. Sie kann näherungsweise aus den Bindungsenergien der beteiligten Niveaus berechnet werden. Für den in Abbildung 2-3a dargestellten Fall ergibt sich:

$$
E_{\text {kin }}=\left(E_{K}-E_{L 1}\right)-E_{L 2}
$$

Die hier eingesetzten Energien sind nicht exakt identisch mit den Niveaus eines freien Atoms, da durch die Bindung im Festkörper die Energieniveaus verschoben werden. Außerdem wird das Augerelektron aus einem schon ionisierten Atom emittiert, wodurch eine höhere Bindungsenergie zu überwinden ist. 


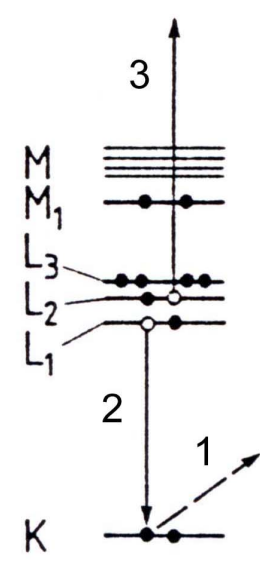

Auger
$K L_{1} L_{2}$

(a)

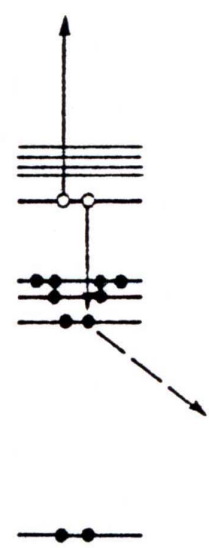

Auger

$L_{1} M_{1} M_{1}$

(b)

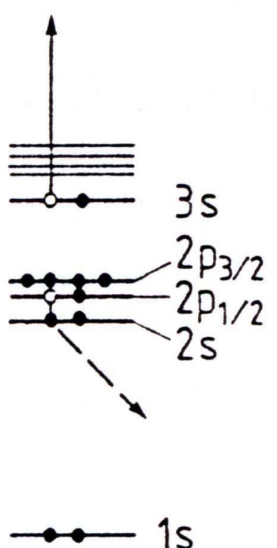

Coster-Kronig

$L_{1} L_{2} M_{1}$

(c)

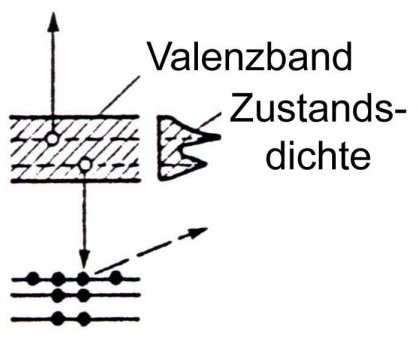

Auger(Solid)

$L_{3} V V$

(d)

Abbildung 2-3: Prinzip der Augerelektronenemission [24]

Nach den in Abbildung 2-3a beteiligten Energieniveaus wird dieser Übergang $\mathrm{KL}_{1} \mathrm{~L}_{2}$ Prozeß genannt. KLL-Prozesse sind neben den LMM- und MNN-Prozessen die am häufigsten auftretenden. Die nach der primären Ionisation entstandene Lücke muß aber nicht wie bei diesen Übergängen durch ein Elektron einer höheren Schale gefüllt werden. Abbildung 2-3c zeigt einen sogenannten Coster-Kronig-Prozeß, bei dem beide Elektronen derselben Schale entstammen (L) und aufgrund der geringeren Energiedifferenz das Augerelektron aus einer Schale näher am Vakuumniveau emittiert wird (M). Bei Metallen können auch Elektronen des Valenzbandes an Auger-Prozessen beteiligt sein, was mit einem $\mathrm{V}$ für das Niveau gekennzeichnet wird (Abbildung 2-3d).

Die von der Probe in das Spektrometer treffenden Augerelektronen werden durch einen Analysator geleitet, der abhängig von der anliegenden Spannung jeweils nur für ein bestimmtes Energieintervall durchlässig ist, und treffen dann auf einen Sekundärelektronenvervielfacher, der für jedes auftreffende Elektron einen meßbaren Spannungspuls abgibt. Die Zahl der Pulse pro Zeiteinheit wird als Maß für die Intensität rechnergestützt aufgezeichnet. Durch Variation der am Analysator anliegenden Spannung kann so ein Spektrum der Elektronenenergien aufgenommen werden.

Typische Energien von Augerelektronen liegen zwischen $50 \mathrm{eV}$ und $1000 \mathrm{eV}$. Elektronen in diesem Energiebereich werden in Festkörpern nach wenigen Monolagen 


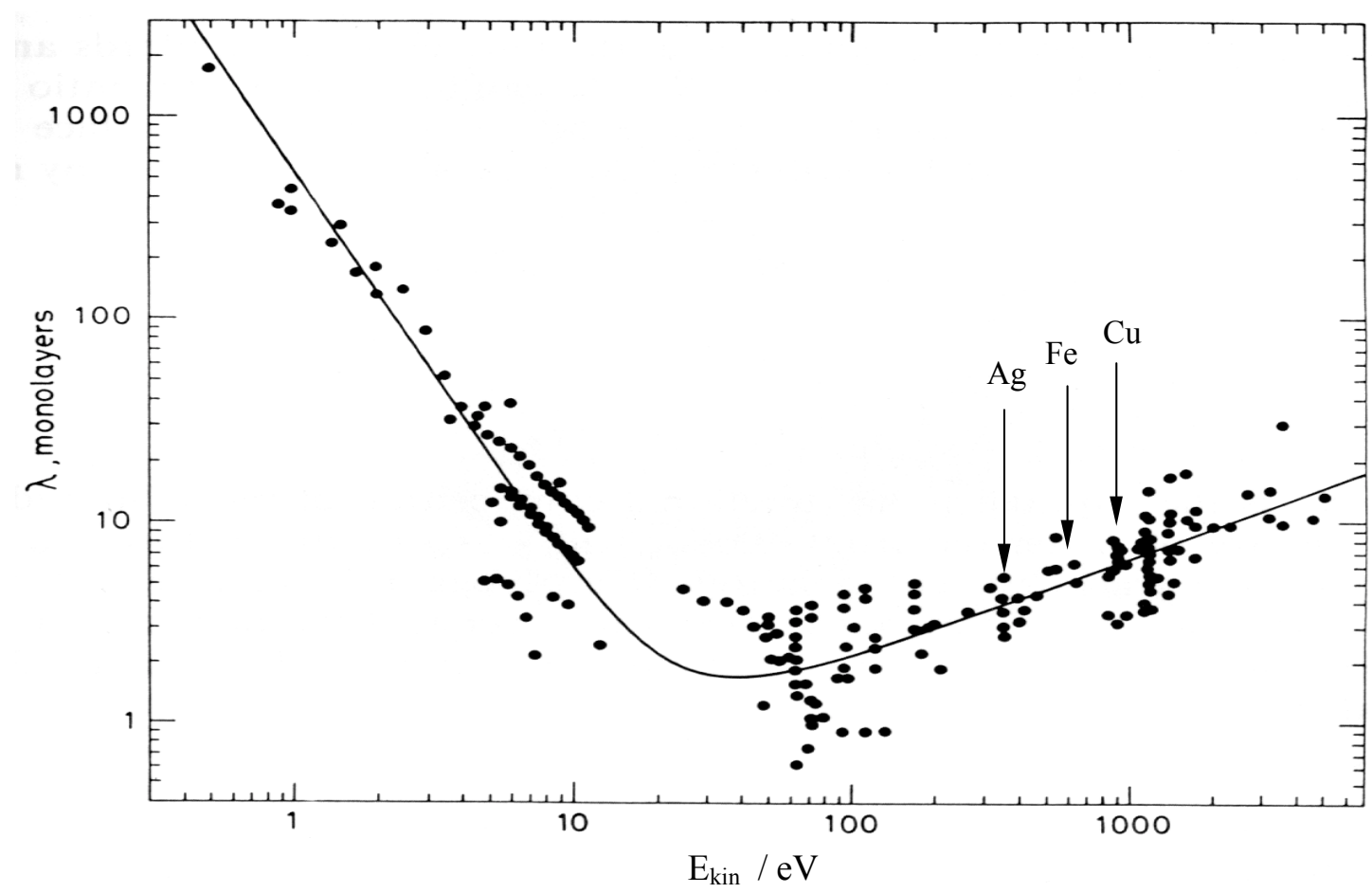

Abbildung 2-4: Mittlere freie Weglänge $\lambda$ für Elektronen in Festkörpern in Abhängigkeit von ihrer kinetischen Energie [24]. Markiert sind die Augerelektronenenergien der in dieser Arbeit untersuchten Elemente Ag, $\mathrm{Fe}$ und $\mathrm{Cu}$.

gestreut und abgebremst. Deshalb können die detektierten Augerelektronen nur aus einer dünnen Oberflächenschicht der Probe stammen. Abbildung 2-4 zeigt eine Auftragung der mittleren freien Weglänge $\lambda$ von Elektronen in Abhängigkeit von ihrer Energie. Mit abnehmender Energie wird $\lambda$ erwartungsgemäß kleiner, erreicht zwischen $10 \mathrm{eV}$ und $100 \mathrm{eV}$ ein Minimum, um daraufhin wieder anzusteigen. Dieser Wiederanstieg ist darauf zurückzuführen, daß die Energie der Elektronen nicht mehr ausreicht, Plasmonen anzuregen, was sonst stark zum Energieverlust der Elektronen beiträgt.

Die Empfindlichkeit der AES beträgt somit maximal 1 bis 2 Monolagen, wenn AugerPeaks unter $100 \mathrm{eV}$ vorhanden sind und ausgewertet werden können. Bei diesen Energien tritt eine sehr starke Emission von Sekundärelektronen auf, die die vergleichsweise schwache Augerelektronen-Emission überdeckt. Si ist ein Element, dessen Konzentration aus diesem Grund nicht quantitativ bestimmt werden konnte. Für die Auger-Übergänge der anderen in dieser Arbeit untersuchten Elemente $\mathrm{Cu}, \mathrm{Ag}, \mathrm{Fe}$ ergeben sich mittlere freie Weglängen bis maximal 7 Monolagen im Fall von $\mathrm{Cu}$. 


\subsection{Röntgendiffraktometrie}

Zur Aufklärung der Kristallstruktur der Filme wurden die Proben mittels Röntgendiffraktometrie in Bragg-Brentano-Geometrie untersucht [25]. Über die BraggBeziehung

$$
n \lambda=2 d \sin \theta
$$

läßt sich bei einer gegebenen Röntgenwellenlänge $\lambda$ ein unter einem Beugungswinkel $\theta$ gemessener Peak der Ordnung $n$ einem Netzebenenabstand $d$ zuordnen, der wiederum für eine bestimmte Kristallrichtung charakteristisch ist. Auf diese Weise kann die Orientierung der Filme senkrecht zur Probenoberfläche bestimmt werden.

Eingesetzt für diese Messungen wurde ein Vierkreis-Diffraktometer vom Typ X-Pert der Firma Philips. Dieses Gerät verwendet Fe-gefilterte Co-K $\mathrm{K}_{\alpha}-$ Strahlung $(\lambda=0,179 \mathrm{~nm})$ und bietet die Möglichkeit, die Probe um einen Winkel $\chi$ aus der $\theta-2 \theta$ Streuebene zu verkippen sowie um einen Winkel $\varphi$ um die eigene Achse zu drehen. Damit wird es möglich, die Textur der Probe zu bestimmen, indem die BraggBedingung für einen zu erwartenden Reflex eingestellt wird (d.h. ein fester Wert für $\theta$ ) und die gestreute Intensität für alle Orientierungen $(\chi, \varphi)$ der Probe gemessen wird. Da bei texturiertem Wachstum die Reflexe bei nur wenigen bekannten Verkippungswinkeln $\chi$ der Probe auftreten können, wurden in dieser Arbeit sog. $\varphi$-Scans durchgeführt, bei denen $\chi$ auf einer zu erwartenden Reflexposition festgehalten und $\varphi$ von $0^{\circ}$ bis $360^{\circ}$ variiert wird. Bei einem in der (111)-Orientierung aufwachsenden fcc-Metall kann man beispielsweise den unter $\chi=54,7^{\circ} \mathrm{zu}$ erwartenden (200)-Reflex ${ }^{1}$ messen. Wächst die Schicht untexturiert auf, bewirkt die statistische Verteilung der Körner eine von $\varphi$ unabhängige, konstante Intensität. Bei einer texturierten Schicht hingegen erhält man Maxima der Intensität für bestimmte Winkel $\varphi$, aus denen die Epitaxiebeziehung zwischen Substrat und Schicht ersichtlich wird.

\subsection{Kleinwinkel-Röntgenstreuung}

Im Unterschied zur Röntgendiffraktometrie im Großwinkelbereich, bei der konstruktive Interferenz der Röntgenstrahlung für Ebenenabstände $d$ im Å-Bereich auftritt, werden

\footnotetext{
${ }^{1}$ Der (100)-Reflex tritt nach den Auswahlregeln für Röntgenstreuung an fcc-Kristallen nicht auf (siehe z. B. [26])
} 

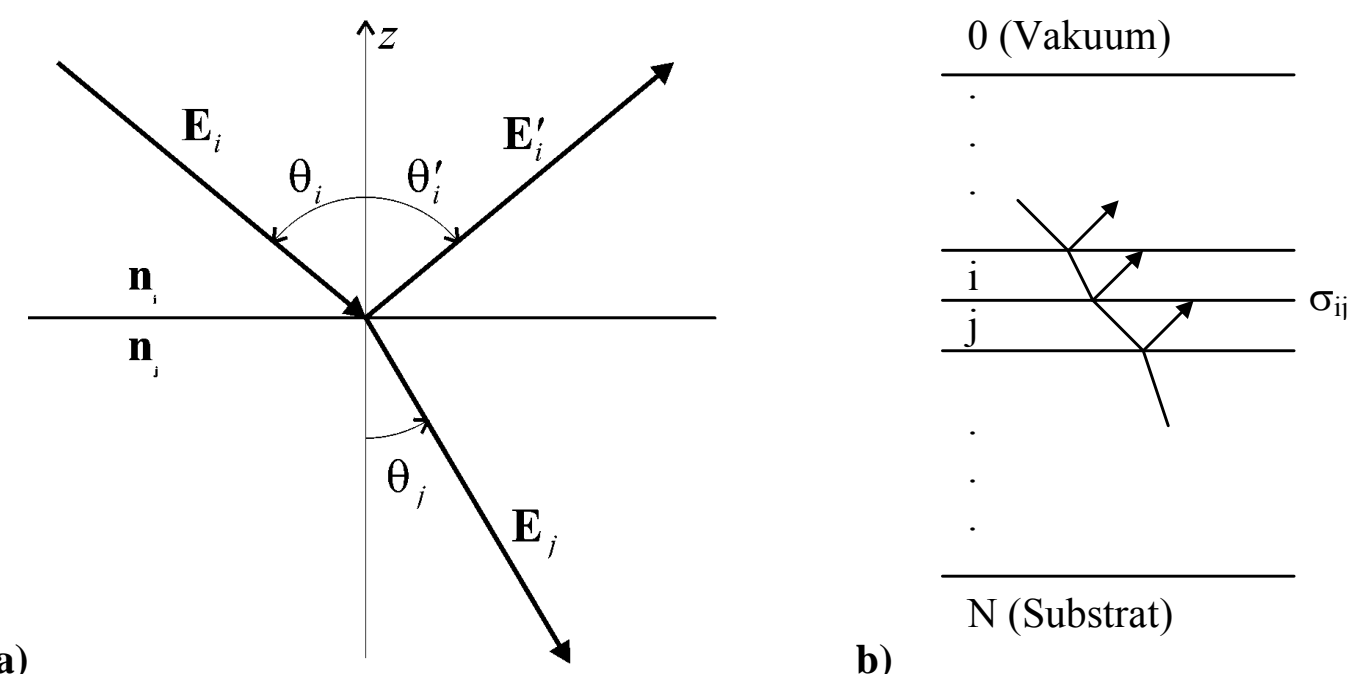

b)

Abbildung 2-5: Prinzipskizze zur a) Streuung an einer Grenzfläche $\left(\theta_{i}=\theta_{i}^{\prime}\right.$ bei spekularer Streuung) zwischen zwei Medien mit unterschiedlichen Brechungsindizes, b) Streuung in einer Schichtfolge (siehe Text)

bei der Kleinwinkelstreuung Einfalls- und Ausfallswinkel $\theta$ soweit verkleinert, daß Interferenz für Abstände bis in den $100 \mathrm{~nm}$-Bereich hinein möglich wird (vgl. (2-3)). Aus der Peaklage läßt sich damit direkt die Schichtdicke, bei Multischichten zusätzlich die Periode bzw. Doppelschichtdicke, bestimmen. Typische Werte für $2 \theta$ sind einige Grad für Doppelschichtperioden von einigen Nanometern und einige zehntel Grad für die Gesamtschichtdicken, die für diese Arbeit zwischen 50 und maximal $300 \mathrm{~nm}$ lagen. Für diese Messungen wurde ebenfalls das oben beschriebene Diffraktometer verwendet, allerdings zusätzlich mit einem Monochromator und Soller-Schlitzen im Sekundärstrahlengang, um die benötigte hohe Auflösung von unter $0,01^{\circ} \mathrm{zu}$ erreichen.

Neben der Schichtdicke läßt sich aus dem genauen Verlauf des Spektrums die Oberflächenrauhigkeit und die Rauhigkeit bzw. Durchmischung der inneren Grenzflächen bestimmen. Allerdings lassen sich diese Größen nicht mehr analytisch berechnen, sondern müssen aus einer Simulation des Strahlenverlaufs in der Probe und der Anpassung des so berechneten Spektrums an das gemessene abgeleitet werden. Für diese Simulation wurde das Softwarepaket IMD/XOP eingesetzt [27]. Die zugrundeliegende Theorie ist in [28] beschrieben und soll hier kurz dargestellt werden.

Zunächst wird die Streuung einer elektromagnetischen Wellenfront an einer Grenzfläche zwischen zwei Medien i und j mit unterschiedlichen komplexen Brechungsindizes $n_{i}, n_{j}$ betrachtet (Abbildung 2-5a), wobei 


$$
n=1-\delta+i \beta
$$

Der Dispersionsteil $\delta$ und der Absorptionsteil $\beta$ sind sowohl vom Material als auch von der Wellenlänge $\lambda$ abhängig. Werte für $\delta$ und $\beta$ sind für viele Materialien und Wellenlängen in IMD in Form einer Datenbank integriert. Für die in dieser Arbeit mittels Röntgenstrahlung untersuchten Metalle sind sie sehr klein (Größenordnung $10^{-5}$ und darunter), so daß $n$ nahe bei 1 liegt.

Fällt die ebene Welle unter dem Winkel $\theta_{i}$ auf die Grenzfläche, so ergibt sich für die gebeugte Welle der Ausfallswinkel $\theta_{j}$ aus dem Snelliusschen Brechungsgesetz:

$$
n_{i} \sin \theta_{i}=n_{j} \sin \theta_{j}
$$

Mit $\delta>0$ wird $\operatorname{Re}(n)<1$, was für den Übergang vom Vakuum zum Festkörper zur Totalreflexion bei flachen Einfallswinkeln führt. Einfalls- und Ausfallswinkel $\theta_{i}, \theta_{j}$ sowie die Brechungsindizes der Medien $n_{i}, n_{j}$ bestimmen die Amplitude des reflektierten $\left(E_{i}^{\prime}\right)$ und des transmittierten $\left(E_{j}\right)$ elektrischen Feldes relativ zur einfallenden Amplitude $E_{i}$ über die Fresnelschen Gleichungen, die für s-polarisierte Strahlung lauten:

$$
\frac{\left|E_{i}^{\prime}\right|}{\left|E_{i}\right|}=\frac{n_{i} \cos \theta_{i}-n_{j} \cos \theta_{j}}{n_{i} \cos \theta_{i}+n_{j} \cos \theta_{j}} \equiv r_{i j}^{s}
$$

und

$$
\frac{\left|E_{j}\right|}{\left|E_{i}\right|}=\frac{2 n_{i} \cos \theta_{i}}{n_{i} \cos \theta_{i}+n_{j} \cos \theta_{j}} \equiv t_{i j}^{s}
$$

Für den Fall p-polarisierter Strahlung gilt:

$$
\frac{\left|E_{i}^{\prime}\right|}{\left|E_{i}\right|}=\frac{n_{i} \cos \theta_{j}-n_{j} \cos \theta_{i}}{n_{i} \cos \theta_{j}+n_{j} \cos \theta_{i}} \equiv r_{i j}^{p}
$$

und

$$
\frac{\left|E_{j}\right|}{\left|E_{i}\right|}=\frac{2 n_{i} \cos \theta_{i}}{n_{i} \cos \theta_{j}+n_{j} \cos \theta_{i}} \equiv t_{i j}^{p}
$$

Über die so definierten polarisationsabhängigen Reflexions- und Transmissionskoeffizienten $r$ und $t$ für die Grenzfläche zwischen zwei Lagen $i$ und $j$ läßt sich nun sukzessive die Reflektivität eines Schichtpaketes aus mehreren Lagen bestimmen 
(Abbildung 2-5b). Angefangen bei der untersten Grenzfläche ergeben sich für die Gesamtreflektivität $r_{i}$ und Transmission $t_{i}$ der $i$-ten Lage:

$$
r_{i}=\frac{r_{i j}+r_{j} e^{2 i \varphi_{i}}}{1+r_{i j} r_{j} e^{2 i \varphi_{i}}}, \quad t_{i}=\frac{t_{i j} t_{j} e^{2 i \varphi_{i}}}{1+r_{i j} r_{j} e^{2 i \varphi_{i}}}
$$

mit $\varphi_{i}=2 \pi d_{i} n_{i} \cos \theta_{i} / \lambda$.

Damit ist die reflektierte Intensität eines Schichtpaketes relativ zur einfallenden Intensität, getrennt nach Polarisationsrichtung (Reflexionskoeffizienten $R^{s}, R^{p}$ ):

$$
R^{s}=\left|r_{0}^{s}\right|^{2} \text { und } R^{p}=\left|r_{0}^{p}\right|^{2}
$$

Da bei dem verwendeten Diffraktometer die Polarisationsrichtungen im einfallenden Röntgenstrahl statistisch verteilt sind und der Detektor für alle Polarisationsrichtungen gleich empfindlich ist, wird über die zu s- und p-polarisierter Strahlung gehörende Intensität gemittelt, so daß sich für den totalen Reflexionskoeffizienten $R$ ergibt:

$$
R=\frac{R^{s}+R^{p}}{2}
$$

Die hier beschriebene Theorie gilt zunächst nur für scharfe Grenzflächen. Nach [29] hat ein nicht abrupter Übergang zwischen den Lagen $i$ und $j$ eine Verringerung des Reflexionskoeffizienten $r_{i j}$ um einen Faktor $w_{i j}$ zur Folge, was mit einer entsprechend höheren diffusen Streuung einhergeht. Insbesondere gilt unter der Annahme, daß das Konzentrations- bzw. Rauhigkeitsprofil der Grenzfläche mit der Gaußschen Fehlerfunktion

$$
p(z)=\frac{1}{\sqrt{2 \pi} \sigma} \int_{-\infty}^{z} e^{-\frac{t^{2}}{2 \sigma^{2}}} d t
$$

beschrieben werden kann (Abbildung 2-6),

$$
w_{i j}=e^{-s_{i j}^{2} \sigma_{i j}^{2} / 2}, \text { mit } s_{i j}=\frac{4 \pi \cos \theta_{i}}{\lambda}
$$

Dabei ist $z$ der Abstand von der „Mitte“ (d. h. $p(0)=1 / 2)$ der Grenzfläche und $\sigma_{i j}$ die mittlere Rauhigkeit bzw. -Durchmischung zwischen den Lagen $i$ und $j$. Ob eine homogene Durchmischung oder eine Grenzflächenrauhigkeit vorliegt, kann durch die spekulare Kleinwinkelstreuung nicht unterschieden werden, da beide eine gleich große 


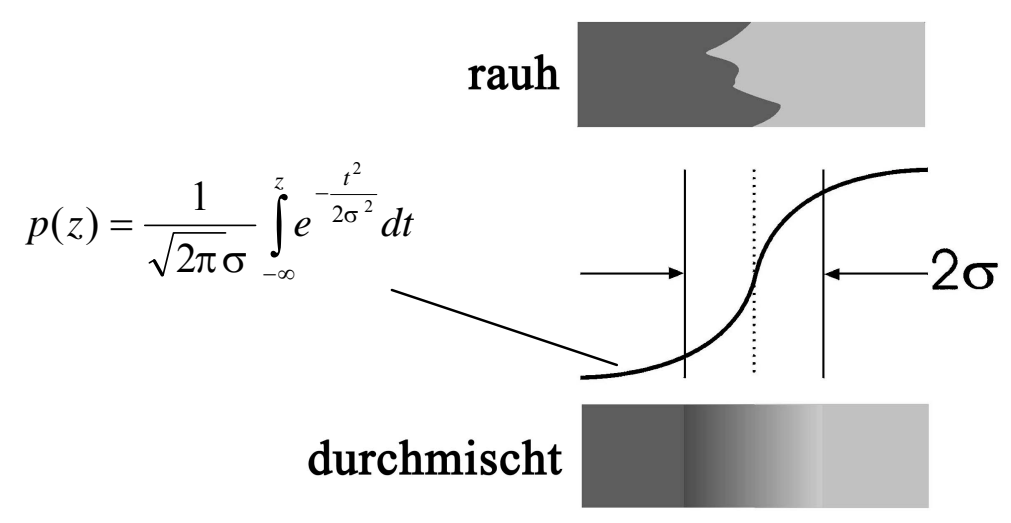

Abbildung 2-6: schematische Darstellung des Grenzflächenprofils p(z), welches als Rauhigkeitsprofil bei rauher Grenzfläche oder als Konzentrationsprofil bei durchmischter Grenzfläche aufgefaßt werden kann

Verringerung des Reflektivitätskoeffizienten bewirken. Deshalb schließt der Begriff „Rauhigkeit“ im folgenden immer auch Durchmischung mit ein.

Um einen Eindruck davon zu bekommen, wie sich Grenzflächenrauhigkeit auf den Intensitätsverlauf in einem Kleinwinkel-Diffraktogramm auswirkt, sind in Abbildung 2-7 simulierte Diffraktogramme für Schichten unterschiedlicher Dicke und Rauhigkeit auf Si dargestellt. Der Konvention in der Literatur entsprechend ist hier $\theta$ der Winkel zwischen einfallendem Röntgenstrahl und der Probenoberfläche. Dies gilt auch für alle in späteren Kapiteln folgenden Röntgendiffraktogramme. Aufgrund der hohen Variation der Intensität über mehrere Größenordnungen wird diese logarithmisch aufgetragen, wobei die einzelnen Kurven zwecks Erhaltung der Übersichtlichkeit jeweils um einen Faktor 100 versetzt sind.

Abbildung 2-7a zeigt zunächst das Diffraktogramm für die Oberfläche eines glatten SiSubstrates $(\sigma=0)$. Die Intensität fällt nach dem kritischen Winkel mit $\theta^{4} \mathrm{ab}$, was sich auch analytisch zeigen läßt [29]. Kurve b zeigt denselben Intensitätsabfall, der allerdings von Oszillationen überlagert ist, die von der $50 \mathrm{~nm}$ dicken $\mathrm{Cu}-\mathrm{Schicht}$ auf dem Si-Substrat herrühren. Der kritische Winkel für Totalreflexion $\theta_{c}$ hängt über

$$
\theta_{c}=\lambda \sqrt{\frac{r_{e} \rho_{e}}{\pi}}
$$

von der Elektronendichte $\rho_{e}$ in der bestrahlten Probe ab [30], wobei $r_{e}=2,818 \cdot 10^{-15} \mathrm{~m}$ der klassische Elektronenradius ist. Die Elektronendichte steigt wiederum mit der Dichte des Materials, weshalb $\theta_{c}$ für $\mathrm{Cu}$ deutlich größer ist als im Fall des Si-Substrates. 
Die Kurven c und d zeigen die Diffraktogramme einer Doppelschicht aus $100 \mathrm{~nm} \mathrm{Cu}$ und $10 \mathrm{~nm} \mathrm{Ag}$ auf Si. Hier läßt sich gut erkennen, wie die kurzwelligen Oszillationen der dicken $\mathrm{Cu}$-Schicht durch die langwelligen Oszillationen der dünneren Ag-Schicht moduliert werden. Der Unterschied in den beiden Kurven liegt in der zugrunde gelegten Schichtfolge: für c wurde die Schichtfolge Si-Cu-Ag verwendet, für d die umgekehrte Reihenfolge Si-Ag-Cu. Es zeigt sich, daß eine veränderte Schichtfolge deutlich unterschiedliche Diffraktogramme zur Folge hat und damit mittels Kleinwinkelstreuung identifizierbar ist.

Ähnlich verhält es sich mit Rauhigkeiten an verschiedenen Stellen im Schichtsystem. Die Diffraktogramme e bis g basieren auf demselben Schichtsystem, wie es für $\mathrm{d}$ angenommen wurde ( $\mathrm{Si}-10 \mathrm{~nm} \mathrm{Ag} \mathrm{-} 100 \mathrm{~nm} \mathrm{Cu}$ ). Zusätzlich wurde eine Rauhigkeit von $\sigma=1 \mathrm{~nm}$ für die Grenzfläche Si-Ag im Fall von e, für f dieselbe Rauhigkeit an der Grenzfläche $\mathrm{Ag}-\mathrm{Cu}$ und im Fall von $\mathrm{g}$ diese Rauhigkeit an der $\mathrm{Cu}$-Oberfläche angenommen. Wiederum unterscheiden sich die Kurven deutlich. Eine Substratrauhigkeit wie bei e führt $\mathrm{zu}$ einer gleichmäßigen Verringerung der Oszillationsamplitude über den gesamten Winkelbereich, wohingegen eine Oberflächenrauhigkeit (Kurve g) eine mit dem Winkel zunehmende Dämpfung der kurzwelligen Oszillationen zur Folge hat. Die durch die glatte Ag-Schicht hervorgerufenen langwelligen Oszillationen bleiben davon weitgehend unberührt. Eine Rauhigkeit an der Ag-Cu-Grenzfläche führt wiederum zu einem anderen Verlauf(f).

Mit diesen Voraussetzungen erweist sich die Kleinwinkel-Röntgenstreuung als eine sehr gute Methode, um die Schichtdicken und Rauhigkeiten individueller Grenzflächen in Doppelschichtsystemen zu bestimmen, indem simulierte Diffraktogramme über die in IMD integrierte numerische Fitprozedur an die Messungen angepaßt werden.

Da die Simulation der Spektren voraussetzt, daß die bestrahlte Fläche immer kleiner ist als die Probe, dürfen die Proben eine gewisse Mindestgröße nicht unterschreiten, um die Vergleichbarkeit der Messung mit der Simulation zu gewährleisten. Diese Größe hängt von Geometrie und Aufbau des verwendeten Spektrometers ab. Für die verwendeten $10 \times 20 \mathrm{~mm}^{2}$ großen Substrate beträgt der Winkel, ab dem die bestrahlte Fläche kleiner wird als die Probe, $\theta=0,5^{\circ}$. Für kleinere Winkel sind Abweichungen zwischen Messung und Simulation zu erwarten, die aber bei metallischen Schichten im Bereich der Totalreflexion liegen (siehe z. B. Abbildung 4-1). 


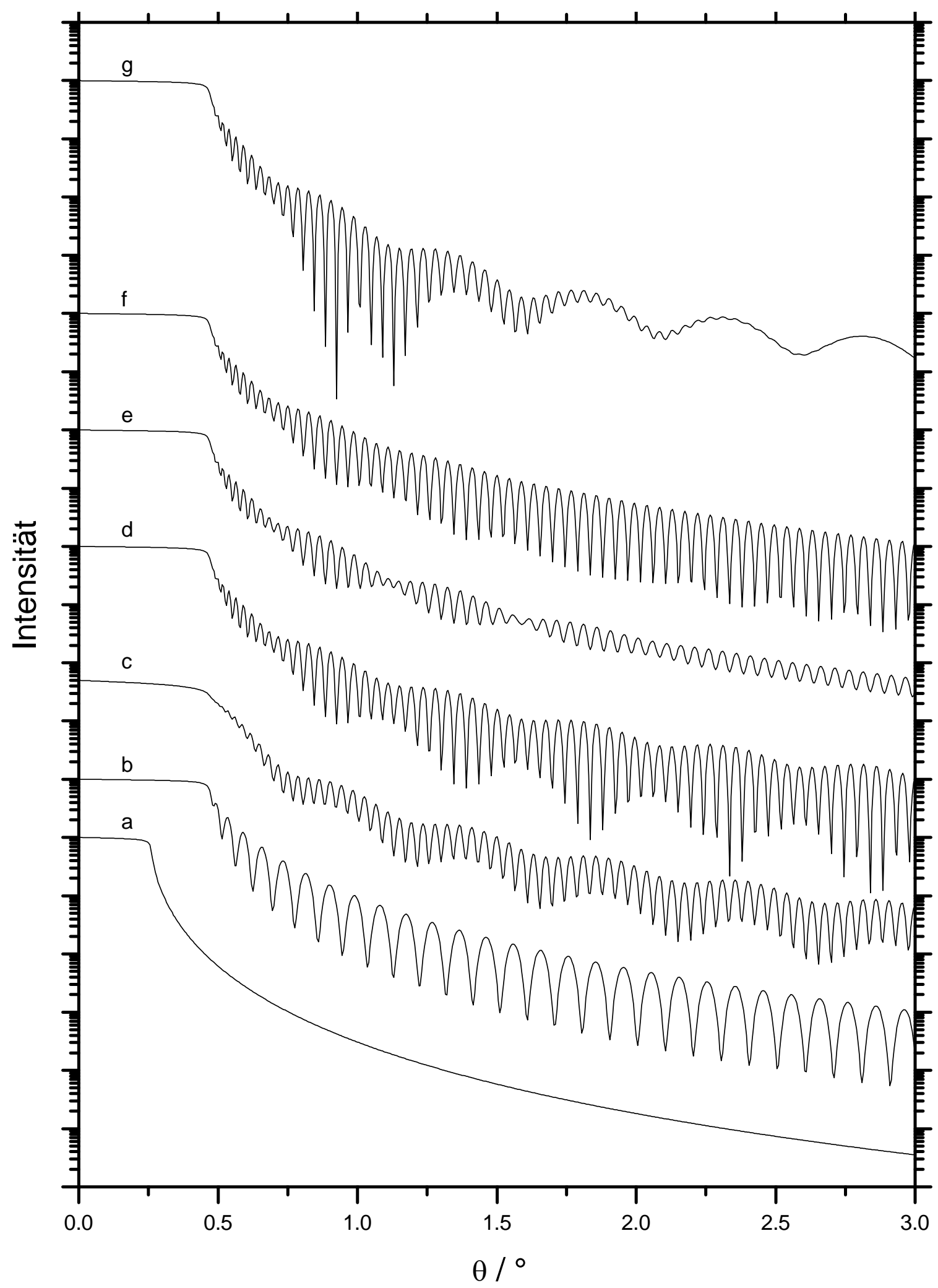

Abbildung 2-7: simulierte Kleinwinkel-Röntgendiffraktogramme; a) Si-Substrat, b) Si$50 \mathrm{~nm} \mathrm{Cu}, \mathrm{c}) \mathrm{Si}-100 \mathrm{~nm} \mathrm{Cu}-10 \mathrm{~nm} \mathrm{Ag}$, d) Si-10 $\mathrm{nm} \mathrm{Ag-100} \mathrm{nm} \mathrm{Cu,} \mathrm{e)} \mathrm{bis} \mathrm{g)} \mathrm{wie} \mathrm{d),}$ aber zusätzlich $1 \mathrm{~nm}$ Grenzflächenrauhigkeit des Si-Substrates (e), der Ag-Schicht (f) bzw. $\operatorname{der} \mathrm{Cu}$-Schicht $(\mathrm{g})$ 


\subsection{Konversionselektronen-Mößbauerspektroskopie}

Die Mößbauerspektroskopie ist eine Methode, mit der sich durch eine hoch präzise Messung bestimmter Energieniveaus im Kern eines Sondenatoms elektrische und magnetische Felder am Ort des Kerns bestimmen lassen. Diese Felder werden von der atomaren Umgebung des Sondenatoms beeinflußt, so daß z. B. eine Fremdatomkonzentration ermittelt werden kann.

Die der Mößbauerspektroskopie zugrunde liegende rückstoßfreie Resonanzabsorption von Kern- $\gamma$-Quanten und ihre Anwendung ist in verschiedenen Publikationen detailliert beschrieben, siehe beispielsweise [31,32]. Hier sollen nur die für das Verständnis der in dieser Arbeit gezeigten Messungen notwendigen Prozesse und der verwendete Aufbau dargestellt werden. Abbildung 2-8 zeigt eine Schemazeichnung zur Funktionsweise der Konversionselektronen-Mößbauerspektroskopie (CEMS), wie sie hier eingesetzt wurde. Ausgangspunkt für die Messung ist eine radioaktive ${ }^{57} \mathrm{Co}$-Quelle $\left(\tau_{1 / 2}=270 \mathrm{~d}\right)$ mit einer Aktivität von etwa $400 \mathrm{MBq}$, in der das Co-Isotop über Elektroneneinfang aus der KSchale zu angeregtem ${ }^{57} \mathrm{Fe}$ zerfällt. Der ${ }^{57} \mathrm{Fe}$-Kern relaxiert daraufhin mit einer Wahrscheinlichkeit von 89\% über den ersten angeregten Zustand in den Grundzustand, in den restlichen 11\% der Fälle direkt in den Grundzustand [33]. Die Energiedifferenz von $14,4 \mathrm{keV}$ beim Übergang vom ersten angeregten Zustand in den Grundzustand wird mit einer Wahrscheinlichkeit von etwa $10 \%$ durch ein $\gamma$-Quant freigesetzt [33]. Die Impulserhaltung bedingt, daß bei einem freien Atom ein Teil der Energie als Rückstoßenergie an den emittierenden Atomkern übertragen wird. Die kinetische Energie des $\gamma$-Quants ist um diesen Rückstoßbetrag verringert und außerdem durch die thermische Bewegung der Atome verbreitert. Die Rückstoßenergie und die thermische Verbreiterung bei Raumtemperatur sind sehr viel größer als die natürliche Linienbreite, die sich aus der mittleren Lebensdauer des ersten angeregten Niveaus von $141 \mathrm{~ns} z \mathrm{zu}$ $4,7 \cdot 10^{-9} \mathrm{eV}$ ergibt. Ist das emittierende Atom jedoch in einer Kristallstruktur gebunden, ist dessen thermische Bewegung durch das Phononenspektrum des Kristalls vorgegeben. Dabei kann die mittlere Geschwindigkeit eines einzelnen Atoms sehr klein sein, und der Rückstoßimpuls wird mit einer gewissen Wahrscheinlichkeit (dem sog. Debye-Waller-Faktor) vom Kristall als ganzes aufgenommen. Aufgrund der hohen Masse eines makroskopischen Festkörpers im Vergleich zum einzelnen Atom ist die in diesem Fall auf den Kristall übertragene Energie verschwindend klein. Die rückstoßfrei emittierten Photonen besitzen eine Energieverteilung, die der natürlichen Linienbreite 


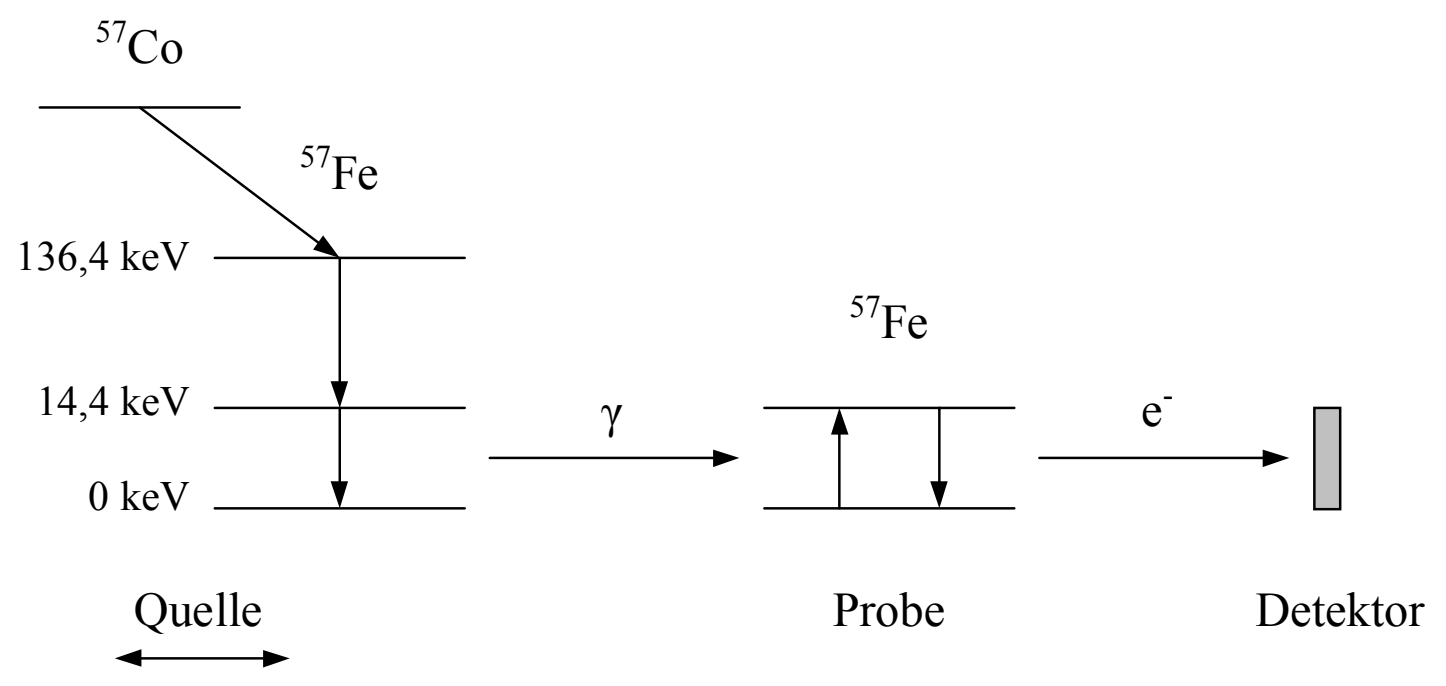

Abbildung 2-8: Schemaskizze der bei der CEMS ablaufenden Prozesse

des Kernübergangs entspricht. Der hier diskutierte Übergang im ${ }^{57} \mathrm{Fe}$ hat einen sehr hohen Debye-Waller-Faktor von 0,76 bei Raumtemperatur [34], weshalb sich dieses Isotop für die Mößbauerspektroskopie besonders anbietet.

Die von der Quelle emittierten $\gamma$-Quanten treffen auf die Probe, in der ebenfalls ${ }^{57} \mathrm{Fe}$ Atome enthalten sind. Die Energieniveaus der ${ }^{57} \mathrm{Fe}-\mathrm{Kerne}$ in der Probe können aus

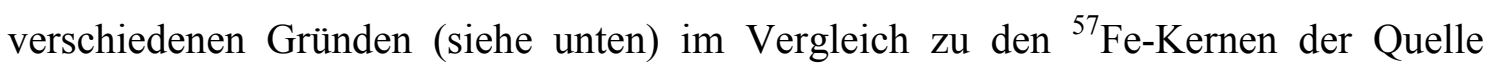
verschoben sein, so daß keine Absorption der $\gamma$-Quanten möglich ist. Um eine Absorption $\mathrm{zu}$ ermöglichen, muß die Energie $E_{0}$ der emittierten Photonen variiert werden. Dies wird durch die Bewegung der Quelle mit einer definierten Geschwindigkeit $v$ und dem daraus resultierenden Dopplereffekt erreicht:

$$
E=E_{0}\left(1+\frac{v}{c}\right)
$$

Dabei ist $c$ die Lichtgeschwindigkeit. Typische Geschwindigkeiten liegen im Bereich von einigen $\mathrm{mm} / \mathrm{s}$.

Die durch die Absorption gebildeten angeregten ${ }^{57} \mathrm{Fe}-$ Kerne können ihre Energie wieder als Photon oder aber in Form von Elektronen abgeben, die aus den inneren Schalen der Atome emittiert werden. Diese Konversionselektronen wurden in einem $\mathrm{He} / \mathrm{CH}_{4}$ Proportionalzählrohr [35] detektiert und ihre Zahl in Abhängigkeit von der Geschwindigkeit der Quelle gespeichert. Die kinetische Energie der Konversionselektronen liegt im keV-Bereich, so daß sie Fe-Schichten von etwa $100 \mathrm{~nm}$ 


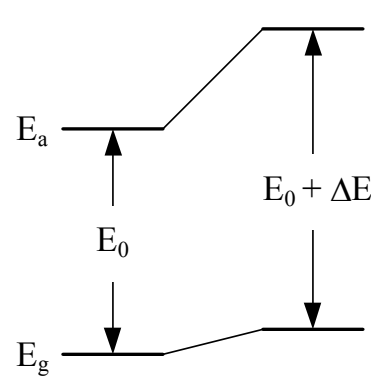

a)

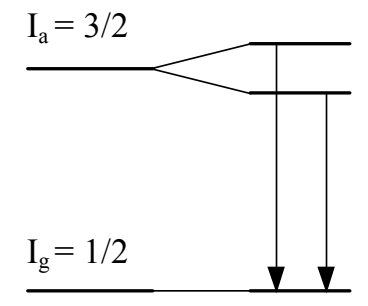

b)

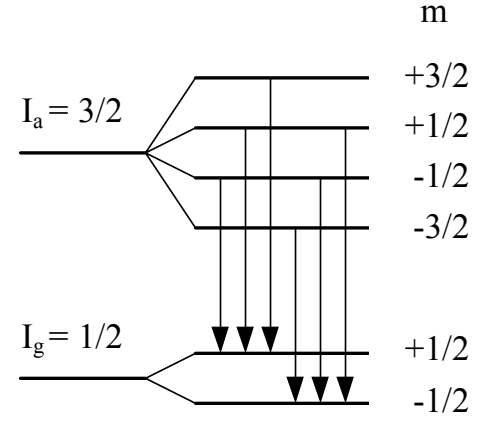

c)

Abbildung 2-9: Für die Mößbauerspektroskopie wichtige Verschiebungen und Aufspaltungen der Energieniveaus; a) Isomerieverschiebung, b) elektrische Quadrupolaufspaltung, c) magnetische Hyperfeinfeldaufspaltung

bis $400 \mathrm{~nm}$ durchdringen können [36]. Die CEMS eignet sich daher gut für die Untersuchung dünner Filme.

Ursache für eine Verschiebung und Aufspaltung der Energieniveaus im ${ }^{57} \mathrm{Fe}-\mathrm{Kern}$ sind Wechselwirkungen des Kerns mit umgebenden elektrischen und magnetischen Feldern. Durch die Coulomb-Wechselwirkung der Kernladung mit der Elektronendichte am Kern verschieben sich die Niveaus im Vergleich $\mathrm{zu}$ einer Punktladung (Isomerieverschiebung - Abbildung 2-9a). Um einen Nullpunkt definieren zu können, werden alle Isomerieverschiebungen relativ zu $\alpha$-Fe angegeben. Dazu wurde mit einer $25 \mu \mathrm{m}$ dicken $\alpha$-Fe-Folie bei Raumtemperatur kalibriert.

Die Wechselwirkung des elektrischen Quadrupolmoments des Kerns mit einem Gradienten des elektrischen Feldes am Kern führt $\mathrm{zu}$ einer Aufspaltung der Energieniveaus (elektrische Quadrupolaufspaltung - Abbildung 2-9b). Im Fall von ${ }^{57} \mathrm{Fe}$ spaltet nur der angeregte Zustand auf, was zu einem Mößbauerspektrum mit zwei Linien führt. Der elektrische Feldgradient entsteht durch eine asymmetrische Ladungsverteilung um den Kern, welche wiederum durch eine nichtkubische bzw. verzerrt kubische Gitterstruktur oder eine asymmetrische Anordnung von Fremdatomen im Fe-Gitter verursacht werden kann.

Wenn am Ort des Kerns ein magnetisches Feld existiert, führt dieses über die Wechselwirkung mit dem magnetischen Moment des Kerns zur sog. magnetischen Hyperfeinfeldaufspaltung (Abbildung 2-9c). Ein Energieniveau spaltet dabei 
entsprechend dem Drehimpuls $\mathrm{I}$ des Kerns in diesem Zustand in $\mathrm{m}=2 \mathrm{I}+1$ Unterniveaus auf. Beim ${ }^{57} \mathrm{Fe}$ sind das zwei Unterniveaus für den Grundzustand $\left(I_{g}=1 / 2\right)$ und vier Unterniveaus für den angeregten Zustand $\left(I_{a}=3 / 2\right)$, zwischen denen sechs Übergänge möglich sind $(\Delta \mathrm{m}=-1,0,+1$ muß erfüllt sein $){ }^{2}$

Im allgemeinen ist die magnetische Hyperfeinfeldaufspaltung deutlich stärker als die elektrische Quadrupolaufspaltung, da in $\alpha$-Fe durch die ferromagnetische Ausrichtung der Spins ein starkes inneres Magnetfeld von etwa $32 \mathrm{~T}$ vorherrscht [34]. Durch die Zugabe von nicht ferromagnetisch ordnenden Elementen, wie z. B. Ag, wird dieses Feld reduziert, was durch die entsprechende Verringerung der Aufspaltung der Linien im Mößbauerspektrum sichtbar wird. Auf diese Weise lassen sich Fremdatomkonzentrationen in der Umgebung der ${ }^{57} \mathrm{Fe}$-Atome bestimmen.

\footnotetext{
${ }^{2}$ Um eine Quadrupol- und Hyperfeinfeldaufspaltung der ${ }^{57} \mathrm{Fe}$-Atome in der Quelle zu verhindern, ist das ${ }^{57} \mathrm{Co}$ in eine kubische, unmagnetische Rh-Matrix eingebettet.
} 


\section{Ergebnisse der in-situ-Untersuchungen}

\subsection{Messungen der Depositionsrate}

Die Ratenmessungen werden hier als eigenständiger Teil behandelt, weil ihre Ergebnisse außer der Depositionsrate selbst aufgrund eines nichtlinearen Verhaltens an den Grenzflächen zusätzliche Informationen über das Wachstum laserdeponierter Schichtsysteme liefern. Als Beispiel zeigt Abbildung 3-1 den Verlauf der Schichtdicke mit der Laserpulszahl für zwei Ag-Schichten, die bei unterschiedlichen Laserenergiedichten auf $\mathrm{Cu}$ deponiert wurden, sowie den umgekehrten Fall der Deposition von $\mathrm{Cu}$ auf $\mathrm{Ag}$. Der zu erwartende lineare Anstieg tritt erst nach einer gewissen Anzahl von Pulsen ein. Für Ag ist der Anstieg und damit die Depositionsrate zunächst höher als im Gleichgewichtszustand. Bei $\mathrm{Cu}$ ist der umgekehrte Fall zu beobachten: Die Depositionsrate ist zum Beginn der Deposition auf Ag sehr klein bzw. negativ. Dieser Effekt tritt nur bei der Deposition mit energiereichen Teilchen auf. Neben Atomen mit kinetischen Energien um $10 \mathrm{eV}$ treffen bei der PLD auch Ionen mit einer breiten Energieverteilung um $100 \mathrm{eV}$ auf dem Substrat auf [21]. Diese Energien reichen aus, um schon deponiertes Material wieder abzutragen [10]. Bei der Deposition

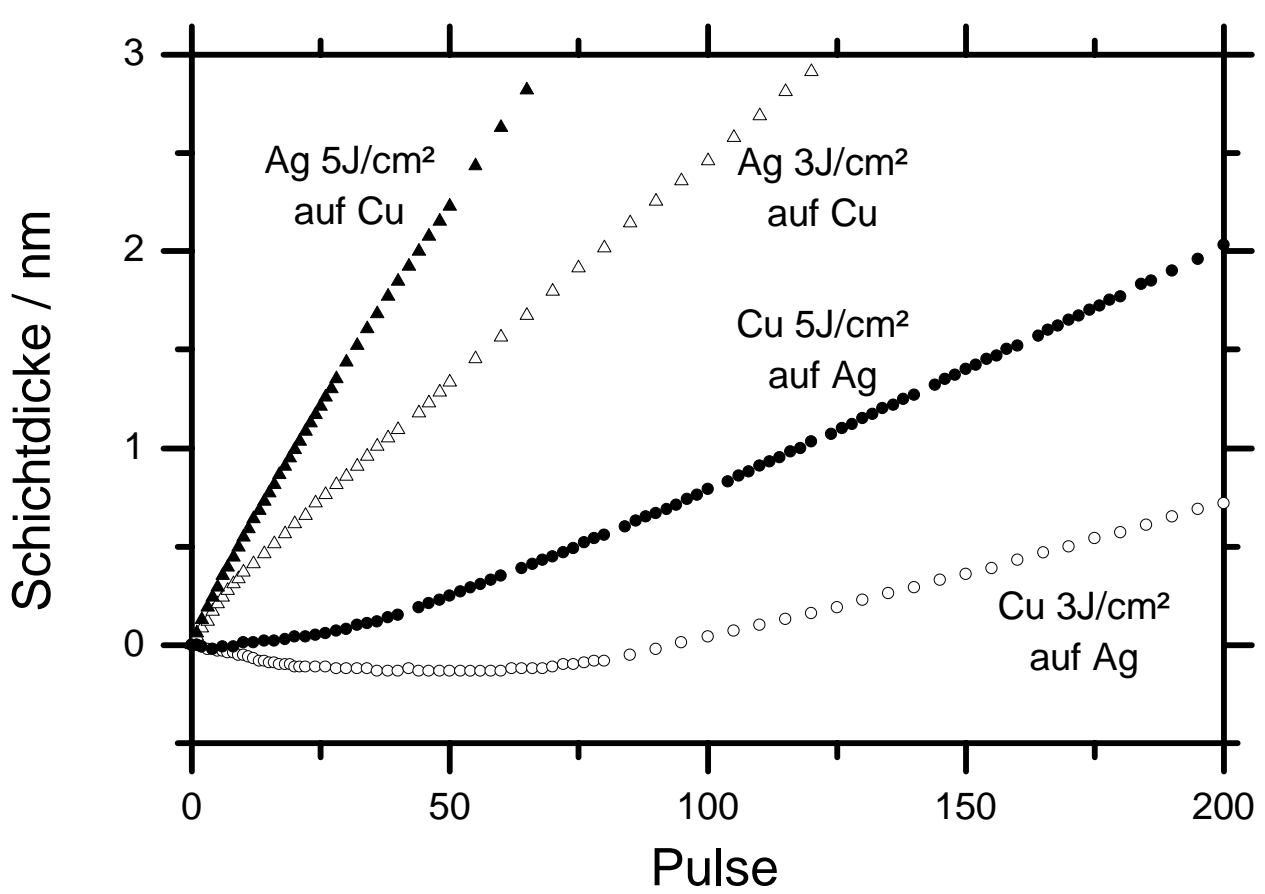

Abbildung 3-1: Schichtdicke in Abhängigkeit von der Pulszahl für die Deposition von $\mathrm{Ag}$ auf $\mathrm{Cu}$ und $\mathrm{Cu}$ auf $\mathrm{Ag}$ bei verschiedenen Energiedichten 
eines Materials auf sich selbst tritt ein Gleichgewichtszustand aus deponierten und wieder abgetragenen Teilchen auf, was $\mathrm{zu}$ der gemessenen konstanten effektiven Depositionsrate führt.

Zur quantitativen Beschreibung des Wiederabtrags dient der Sputteryield $Y_{A-B}$, der aussagt, wie viele Atome des Elementes A im Mittel durch ein auftreffendes Atom B abgetragen werden. In [22] wurden die Eigensputteryields durch Deposition auf einen Ratenmonitor unter verschiedenen Verkippungswinkeln bestimmt. Der mittlere Eigensputteryield von Ag auf Ag bei einer Energiedichte auf dem Target von $5 \mathrm{~J} / \mathrm{cm}^{2}$ beträgt $Y_{A g-A g}=0,55$ und für $\mathrm{Cu}$ auf $\mathrm{Cu} Y_{C u-C u}=0,38$. Wird ein fremdes Element auf die Oberfläche deponiert, beispielsweise $\mathrm{Cu}$ auf $\mathrm{Ag}$, beeinflußt der Fremdsputteryield $Y_{A g-C u}$ die Depositionsrate. Dieser kann vom Eigensputteryield stark abweichen. Bei der Deposition von $\mathrm{Cu}$ auf $\mathrm{Ag}$ ist er deutlich höher (siehe unten) als der Eigensputteryield des $\mathrm{Cu}$. Da außerdem die Atommasse des Ag höher ist als die des $\mathrm{Cu}$, wird in diesem Fall mehr Masse abtragen als deponiert, was sich in der beobachteten negativen Depositionsrate niederschlägt. Erst nach Ausbildung einer geschlossen $\mathrm{Cu}-\mathrm{Schich}$ t wird eine konstante Depositionsrate und ein linearer Anstieg der Schichtdicke erreicht. Im Fall der Deposition von $\mathrm{Ag}$ auf $\mathrm{Cu}$ ist es umgekehrt. Hier sputtert das $\mathrm{Ag}$ das $\mathrm{Cu}$ schwächer als sich selbst und führt so zu einer höheren effektiven Depositionsrate, solange sich noch keine geschlossene Ag-Schicht ausgebildet hat.

Für eine quantitative Beschreibung der gemessenen Depositionsrate $R$ an einer Grenzfläche werden $n_{A}$ Teilchen der Masse $m_{A}$ betrachtet, die auf eine Oberfläche aus A- und B-Atomen auftreffen und diese mit einem Sputteryield von $Y_{A-A}$ bzw. $Y_{B-A}$ pro auftreffendem Atom wieder abtragen. Die A- und B-Atome liegen in den Konzentrationen $c_{A}$ und $c_{B}$ vor, wobei $c_{A}+c_{B}=1$, und es wird angenommen, daß die Sputteryields der Einzelelemente unabhängig von der Konzentration sind, in der sie vorliegen. Diese Betrachtung führt zu folgender Bilanzgleichung für die Depositionsrate $R\left(c_{A}\right)[9]:$

$$
R\left(c_{A}\right)=n_{A}\left(m_{A}-m_{A} c_{A} Y_{A-A}-m_{B}\left(1-c_{A}\right) Y_{B-A}\right)
$$

Definiert man mittels

$$
R_{B-A}=R(0)=n_{A}\left(m_{A}-m_{B} Y_{B-A}\right)
$$

die Depositionsrate auf die reine B-Oberfläche (zu Beginn der Deposition) und mittels

$$
R_{A-A}=R(1)=n_{A} m_{A}\left(1-Y_{A-A}\right)
$$


die Rate bei Deposition auf die reine A-Oberfläche (am Ende der Deposition), kann der Fremdsputteryield $Y_{B-A}$ nach Umstellung von (3-1) folgendermaßen dargestellt werden:

$$
Y_{B-A}=\left(1-\frac{R_{B-A}}{R_{A-A}}\left(1-Y_{A-A}\right)\right) \frac{m_{A}}{m_{B}}
$$

Für die Deposition bei einer Energiedichte von $5 \mathrm{~J} / \mathrm{cm}^{2}$ ergeben sich Werte von $Y_{C u-A g}=0,28, Y_{A g-C u}=0,66$, was recht gut mit den in [22] bestimmten Werten von 0,24 und 0,69 übereinstimmt. Für die Energiedichte von $3 \mathrm{~J} / \mathrm{cm}^{2}$ sind zwar die Eigensputteryields und damit auch die Fremdsputteryields nicht bekannt, es läßt sich aber aufgrund der stärker ausgeprägten Schichtdickenabnahme bei Deposition von $\mathrm{Cu}$ auf Ag (Abbildung 3-1) davon ausgehen, daß in diesem Fall der Unterschied zwischen Fremd- und Eigensputteryield größer geworden ist, d.h. $Y_{A g-C u}$ noch sehr hoch und $Y_{C u-C u}$ deutlich verringert ist.

Stellt man (3-1) nach der Konzentration $c_{A}$ des deponierten Elementes um und ersetzt mit Hilfe von (3-2) und (3-3) die Sputteryields $Y_{B-A}$ und $Y_{A-A}$ durch die Raten $R_{B-A}$ und $R_{A-A}$, ergibt sich:

$$
c_{A}=\frac{R-R_{B-A}}{R_{A-A}-R_{B-A}}
$$

Diese Depositionsraten können durch Ableitung der in Abbildung 3-1 dargestellten Daten berechnet werden. ${ }^{3}$ Die sich aus (3-5) ergebenden Konzentrationsverläufe über die $\mathrm{Cu}$-Ag-Grenzfläche sind in Abbildung 3-2 dargestellt. Die Pulszahl wurde hier über die Gleichgewichtsraten $R_{C u-C u}$ bzw. $R_{A g-A g}$ in eine deponierte Dicke umgerechnet. Durch die Differenzierung und Glättung der Schichtdickenwerte, die für diese Operation eine zu geringe Auflösung besitzen, und die während der Deposition leicht schwankenden Depositionsraten sind die so erhaltenen Konzentrationsverläufe mit einer hohen Unsicherheit belastet. Qualitativ läßt sich aber feststellen, daß bei der Deposition von $\mathrm{Cu}$ auf $\mathrm{Ag}$ die Konzentrationskurve flacher verläuft als im umgekehrten Fall, die Grenzfläche also stärker durchmischt ist. Dies bestätigt Ergebnisse aus früheren Messungen [11].

3 Die berechneten Größen sind Schichtdickenraten, obwohl Masseraten gefordert sind. Die daraus bestimmte Konzentration $c_{A}$ bleibt aber davon unberührt, da sich der konstante Faktor in (3-5) herauskürzt. 


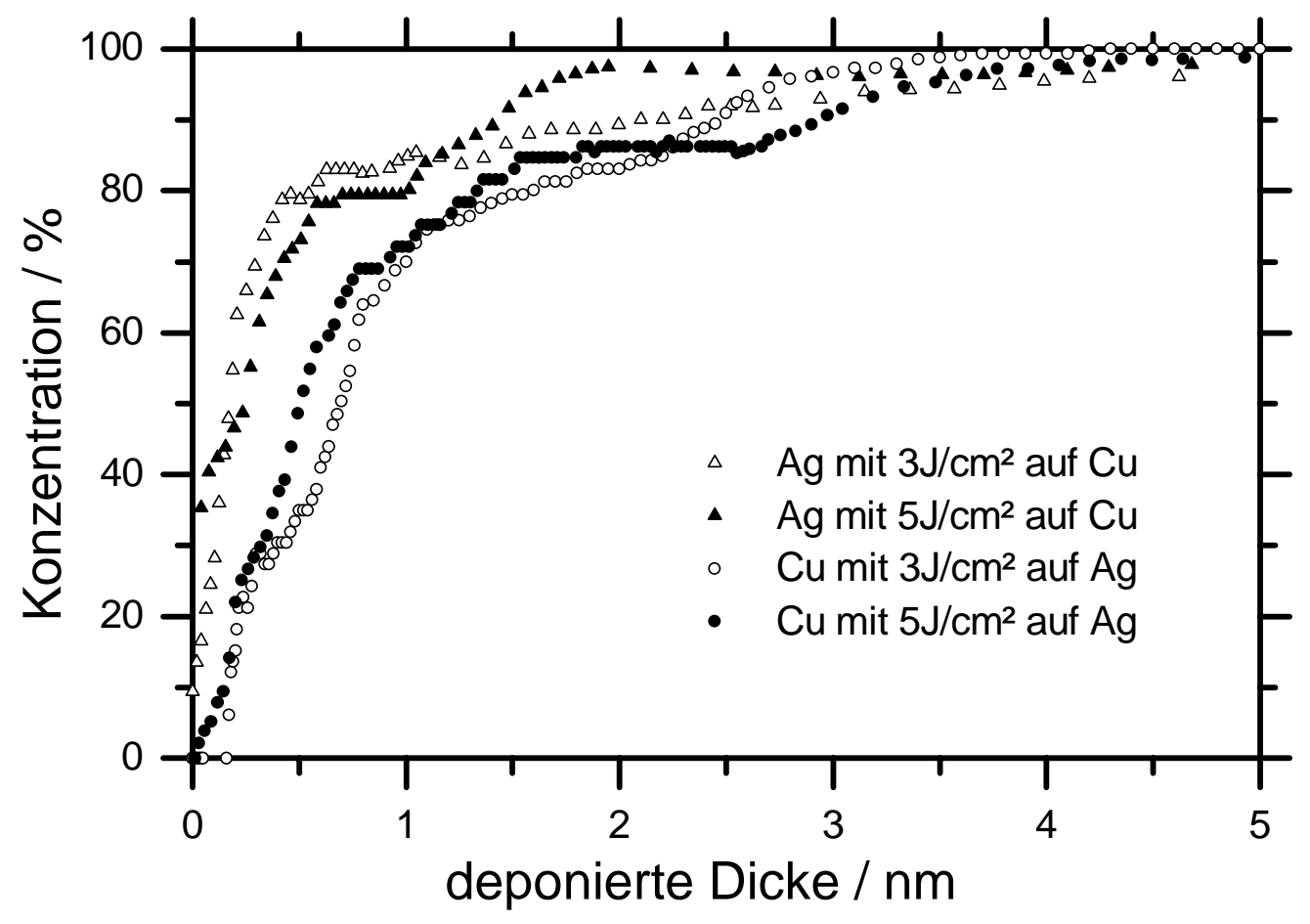

Abbildung 3-2: aus der Depositionsrate bestimmter Konzentrationsverlauf des deponierten Elementes für das System $\mathrm{Cu}-\mathrm{Ag}$

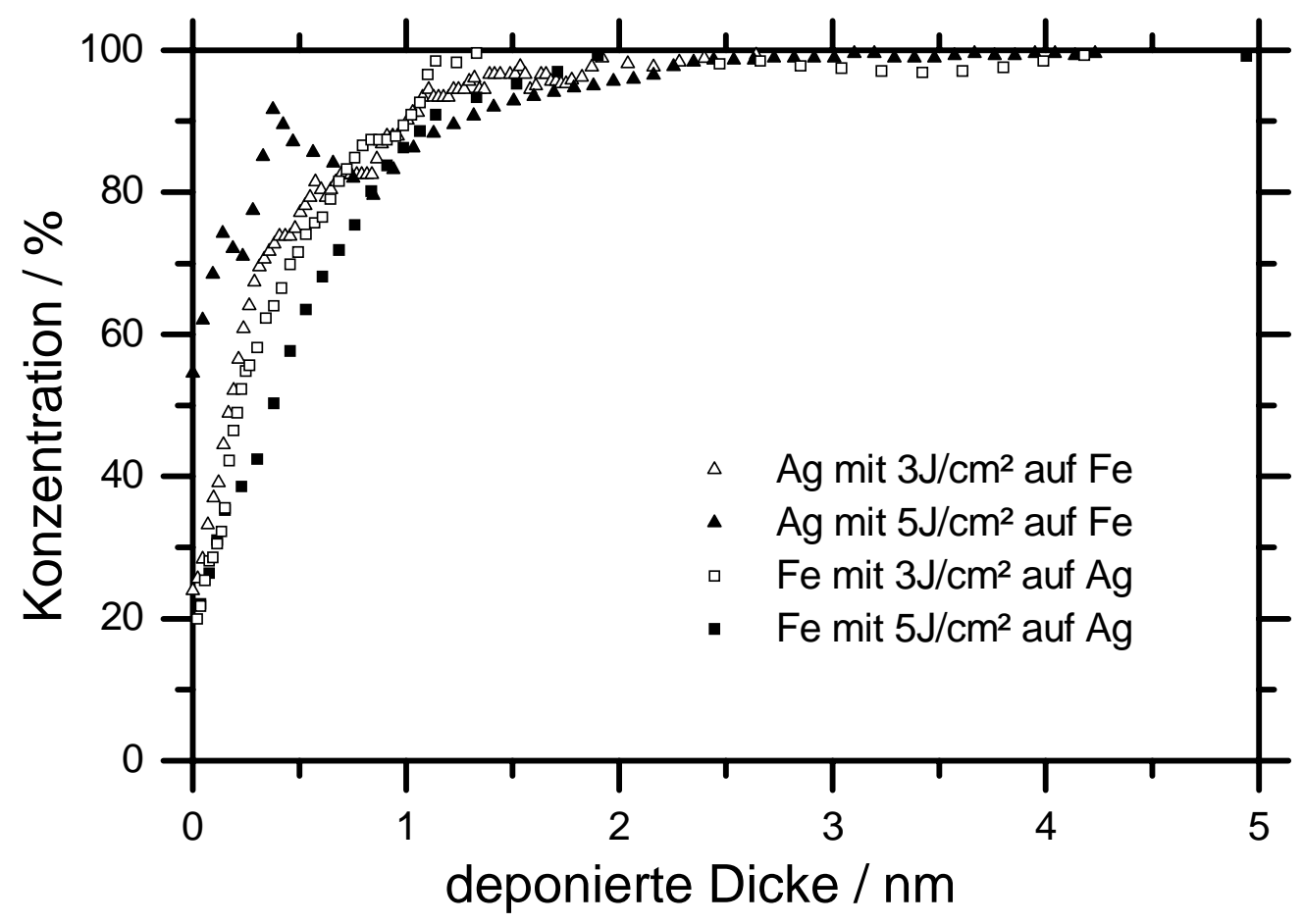

Abbildung 3-3: aus der Depositionsrate bestimmter Konzentrationsverlauf des deponierten Elementes für das System Fe-Ag 
Neben $\mathrm{Cu}-\mathrm{Ag}$ wurden die Depositionsraten von Fe und Ag bei gegenseitiger Deposition ausgewertet. Bei einer Energiedichte von $5 \mathrm{~J} / \mathrm{cm}^{2}$ besitzt $\mathrm{Fe}$ einen kleinen Eigensputteryield von $Y_{\mathrm{Fe}-\mathrm{Fe}}=0,17$ [22] und bewirkt gleichzeitig ein starkes Sputtern der Ag-Oberfläche $\left(Y_{A g-F e}=0,65\right)$, was zu einem ähnlichen Verhalten wie im System $\mathrm{Cu}-\mathrm{Ag}$ führt. Bei Deposition von $\mathrm{Ag}$ auf Fe ist kein Sputtern zu beobachten $\left(Y_{F e-A g}=0\right)$. Die Auswertung der Schichtdickenmessungen nach (3-5) führt zu den in Abbildung 3-3 dargestellten Konzentrationsverläufen. Wie im Fall von $\mathrm{Cu}-\mathrm{Ag}$ bewirken Schwankungen in der Depositionsrate einen relativ hohen Fehler bei der Ableitung der Rate nach der Schichtdicke und damit in der berechneten Konzentration. Trotzdem läßt sich feststellen, daß die Ag- bzw. Fe-Oberfläche nach 2 nm vollständig mit Fe bzw. Ag bedeckt ist. Im Fall von $\mathrm{Cu}-\mathrm{Ag}$ liegt dieser Wert bei etwa 3 bis $4 \mathrm{~nm}$.

In beiden Fällen ist kein Einfluß der bei der Deposition verwendeten Energiedichte auf die Oberflächenkonzentration $\mathrm{zu}$ beobachten, die Konzentrationsverläufe sind im Rahmen der Meßgenauigkeit identisch.

\subsection{Augerelektronenspektroskopie}

Eine direktere Methode zur Bestimmung der Oberflächenkonzentration ist die Augerelektronenspektroskopie (AES), die bei unter gleichen Bedingungen aufgenommenen Spektren eine quantitative Analyse erlaubt. Das Intensitätsverhältnis der Augerlinien verschiedener Elemente ist stark vom verwendeten Spektrometer selbst und von apparativen Parametern, wie Anregungsenergie, Auflösung und Arbeitsabstand, abhängig. Deshalb sind nur Spektren vergleichbar, die unter denselben Spektrometereinstellungen aufgenommen wurden. Insbesondere ist es wichtig, die als Konzentrationsstandard dienenden Spektren der Reinelemente unter genau den Bedingungen $\mathrm{zu}$ messen, die auch für die $\mathrm{zu}$ untersuchenden Proben angewendet wurden.

Die Augeremission ist stets von einem starken Untergrund von Elektronen aus anderen Streuprozessen überlagert, weshalb hier zunächst kurz auf diese störenden, aber wichtigen Anteile in der Elektronenintensität eingegangen werden soll. Da die Untergrundintensität nur eine schwache Abhängigkeit von der Elektronenenergie zeigt, wird in der Literatur meist das differenzierte Augerelektronenspektrum dargestellt und die dort beobachteten Peakhöhen werden als Maß für die Konzentration des betreffenden Elementes herangezogen. Da diese aber keine lineare Abhängigkeit von 
der Augerintensität des Elementes zeigen, werden in dieser Arbeit stets die direkten Spektren zur quantitativen Auswertung herangezogen.

\subsubsection{Einzelschichten}

Abbildung 3-4 zeigt das Augerelektronenspektrum einer $\mathrm{Cu}$-Probe, wobei die Elektronen des anregenden Primärstrahls mit einer Energie von $1000 \mathrm{eV}$ auf die Oberfläche auftrafen. Gemessen wurde das Spektrum der emittierten Elektronen im Bereich von $0 \mathrm{eV}$ bis $1000 \mathrm{eV}$ mit einer relativen Energieauflösung von 0,5\%, was zu einer Abflachung des ansonsten steil abfallenden Spektrums führt. Es sind zwei markante Peaks zu erkennen, die das Spektrum bei niedrigen und hohen Energien begrenzen. Der Peak unterhalb von $50 \mathrm{eV}$ ist auf Sekundärelektronen zurückzuführen, die bei Metallen aus dem Leitungsband herausgelöst werden. Der scharfe Peak bei $1000 \mathrm{eV}$ entspricht genau der Anregungsenergie. Er wird durch elastisch gestreute Primärelektronen hervorgerufen. Etwas unterhalb des elastischen Peaks ist ein weiteres kleines Maximum zu erkennen, das ebenfalls von rückgestreuten Elektronen des Primärstrahls verursacht wird. Der Energieverlust ist darauf zurückzuführen, daß bei der Streuung ein Teil der Energie für die Anregung einer kollektiven Schwingung des Elektronengases, eines sogenannten Plasmons, aufgewendet wurde. Der Energieverlust,

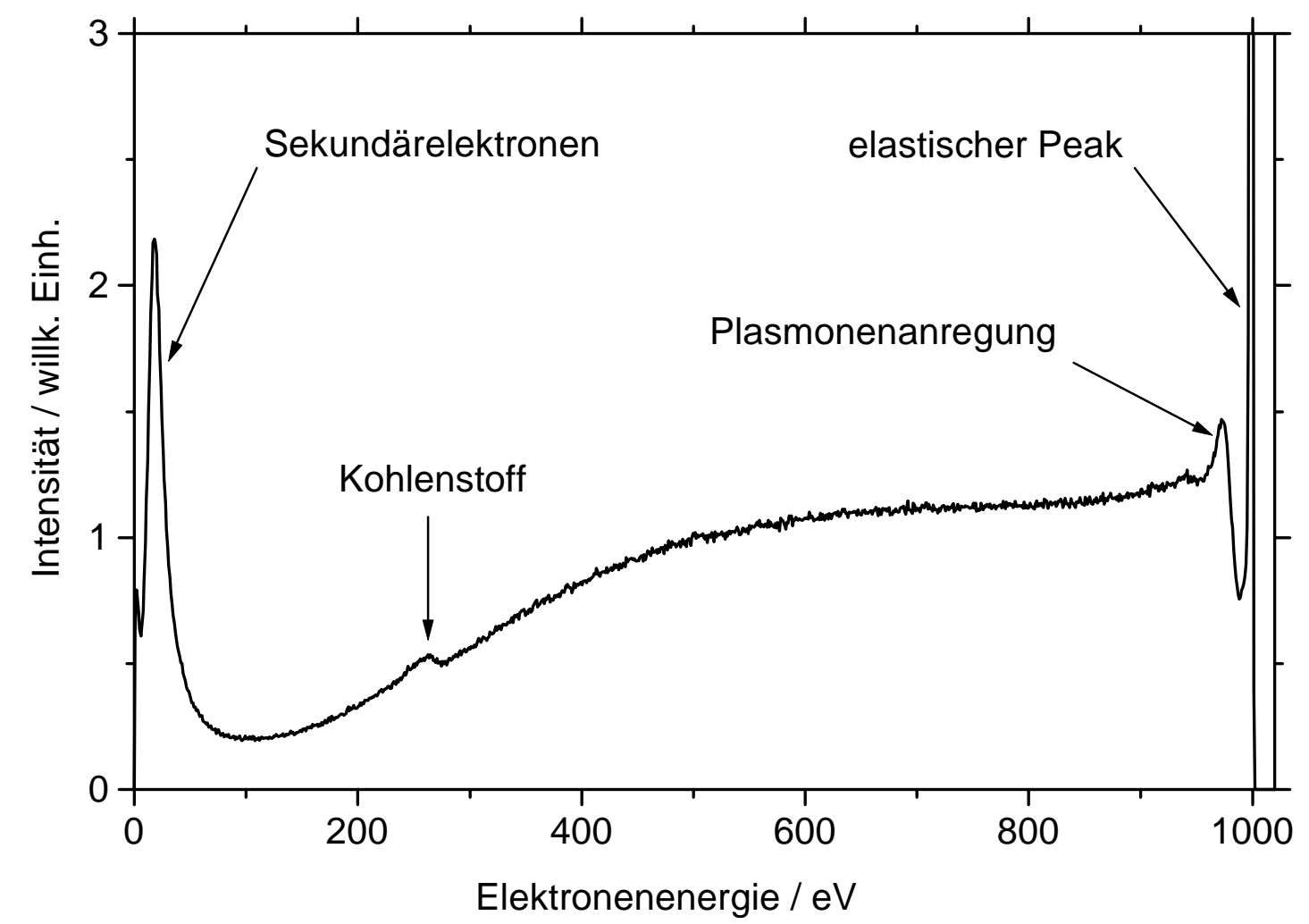

Abbildung 3-4: Augerelektronenspektrum einer Cu-Oberfläche bei einer Anregungsenergie von $1000 \mathrm{eV}$ 
und damit der Abstand zwischen elastischem Peak und dem Plasmonenpeak erster Ordnung, ist charakteristisch für das untersuchte Material. Regt ein einfallendes Elektron mehr als ein Plasmon an, wird es mit entsprechend geringerer Energie zurückgestreut, was zu Plasmonenpeaks höherer Ordnung führt (in Abbildung 3-4 sehr schwach zu erkennen). Die hohe Untergrundintensität im gesamten Spektrum ist auf solche unter starkem Energieverlust gestreuten Elektronen sowie auf Sekundärelektronen zurückzuführen. Die diesem Untergrund überlagerten Augerelektronenpeaks sind vergleichsweise schwach. In diesem Spektrum ist nur der Kohlenstoff-Peak bei $260 \mathrm{eV}$ zu erkennen. C sammelt sich selbst unter UHVBedingungen am Auftreffpunkt des Primärstrahls auf der untersuchten Probe, da der Elektronenstrahl die in geringer Konzentration in der Restatmosphäre vorhandenen Kohlenwasserstoffe crackt und C zurückläßt. Bei kontinuierlicher Deposition einer Schicht mit kurzen (weniger als 2 min) Meßunterbrechungen ist allerdings kein $\mathrm{C}$ nachweisbar.

Die im Spektrum zwischen $750 \mathrm{eV}$ und $900 \mathrm{eV}$ zu erwartenden Augerelektronenpeaks des $\mathrm{Cu}$ treten hier nicht auf, da die Energie des Primärstrahls von $1000 \mathrm{eV}$ zur Anregung nicht ausreicht. Nach [23] ist die intensivste Anregung der Augerlinien im allgemeinen bei etwa dem Fünffachen der Linienenergie zu erwarten. Abbildung 3-5 zeigt die mit einer Primärstrahlenergie von $5000 \mathrm{eV}$ aufgenommenen Augerelektronenspektren der Elemente $\mathrm{Cu}, \mathrm{Ag}$ und Fe. Diese wurden direkt vor der Messung in Form einer etwa $50 \mathrm{~nm}$ dicken Schicht auf den Ratenmonitor deponiert, um eine Kontamination der Oberfläche zu vermeiden. Das Energieintervall zwischen $0 \mathrm{eV}$ und $200 \mathrm{eV}$ der gestreuten Elektronen wurde während der Messung ausgespart, da die hohe Intensität der Sekundärelektronen in diesem Bereich zu einer Überlastung des Detektors geführt hätte. Diese Spektren dienen im folgenden als Eichspektren für die Bestimmung der Oberflächenkonzentration während des Wachstums von $\mathrm{Cu}-\mathrm{Ag}$ - und Fe-Ag-Doppelschichten und müssen deshalb in ihrer absoluten Intensität sehr konstant sein. Um das zu gewährleisten, wurden mehrere Serien von Spektren in der Reihenfolge Ag-Cu-Ag-Fe-Ag aufgenommen und die Serie mit der konstantesten Ag-Intensität als Referenz ausgewählt. Auf diese Weise konnte eine Konstanz von etwa 3\% erreicht werden, trotz der Schwankungen in der Intensität des Primärstrahls, die im Verlauf von Stunden über $10 \%$ betragen können.

Geht man von Reinelementen A, B zu Legierungen aus A und B über, überlagern sich die Augerelektronenspektren der beteiligten Elemente unabhängig voneinander. Die 


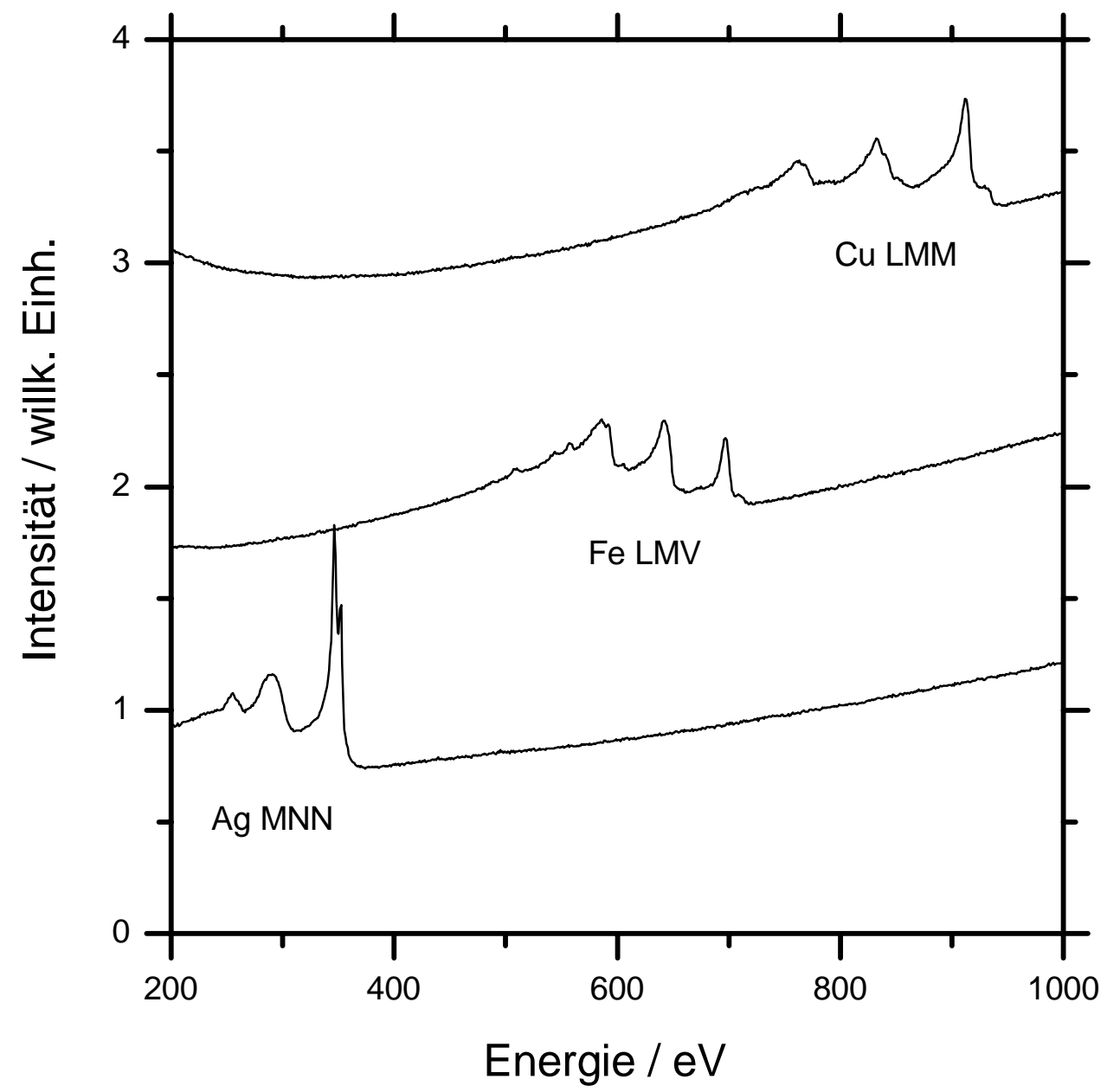

Abbildung 3-5: Augerspektren der Elemente Ag, $\mathrm{Fe}$ und $\mathrm{Cu}$; Die Intensitäten des Fe und des $C u$ sind zugunsten einer besseren Übersichtlichkeit um eine bzw. zwei Einheiten erhöht dargestellt.

Intensität des Gesamtspektrums der Legierung $I_{A B}$ kann so als Linearkombination der Intensitäten der Reinelementspektren $I_{A}, I_{B}$ aufgefaßt werden:

$$
I_{A B}=c_{A} I_{A}+c_{B} I_{B}
$$

Zur Bestimmung der Faktoren $c_{A}, c_{B}$ aus dem Legierungsspektrum wurde eine automatisierte Fit-Prozedur entwickelt, die die relative Intensität der oben bestimmten Eichspektren so lange variiert, bis die beste Übereinstimmung mit dem gemessenen Spektrum erzielt wird. Nach Normierung der Wichtungsfaktoren $c_{A}, c_{B}$ der Eichspektren ergeben diese die gesuchte Konzentration. Da keine Linienüberlagerung im Fall der untersuchten Elemente $\mathrm{Cu}, \mathrm{Ag}$ und $\mathrm{Fe}$ existiert, ist die so bestimmte Konzentration eindeutig. 


\subsubsection{Mehrfachschichten}

An den in Kapitel 3.1 mittels Ratenmessungen untersuchten $\mathrm{Cu}$-Ag- und Fe-AgSchichten wurden in regelmäßigen Abständen während der Deposition Augerelektronenspektren der Oberfläche aufgenommen. Abbildung 3-6 zeigt für die Deposition von $\mathrm{Cu}$ auf $\mathrm{Ag}$ bei einer Energiedichte von $5 \mathrm{~J} / \mathrm{cm}^{2}$ eine Auswahl der Spektren zusammen mit den aus den Eichspektren berechneten Fitkurven. Die gute Übereinstimmung mit den Messungen zeigt, daß der Ansatz der linearen Unabhängigkeit der Reinelementspektren gerechtfertigt ist.

Vergleicht man in Abbildung 3-6 die Gesamtintensität des Augerelektronenspektrums der reinen Ag-Oberfläche und der reinen $\mathrm{Cu}$-Oberfläche mit den Eichspektren in Abbildung 3-5, so ist eine leichte Abnahme der Gesamtintensität zu verzeichnen, was auf die nicht konstante Intensität des Primärstrahls zurückzuführen ist. Im Vergleich zu den Eichspektren kann hier die Gesamtintensität von Spektrum zu Spektrum aber ohne negative Auswirkungen variieren, da die Summe der Konzentrationen aller Elemente für jedes Einzelspektrum auf 100\% normiert wird. Vorausgesetzt werden muß nur eine konstante Primärstrahlintensität innerhalb eines Spektrums, was aufgrund der sehr kurzen Meßzeit von zwei Minuten für ein Spektrum als gegeben angenommen werden kann.

Abbildung 3-6 zeigt, daß selbst nach der Deposition von $3 \mathrm{~nm} \mathrm{Cu}$ noch $25 \%$ der Oberfläche aus Ag besteht. Dies deutet auf eine starke Durchmischung bzw. Inselbildung hin. In Abbildung 3-7 sind die Ergebnisse aller Konzentrationsbestimmungen beim Wachstum von $\mathrm{Cu}$ auf $\mathrm{Ag}$ sowie $\mathrm{Ag}$ auf $\mathrm{Cu}$ dargestellt. Wie schon die Auswertung der Ratenmessungen (Abbildung 3-2) zeigt auch die Konzentrationsbestimmung mittels AES eine stärkere Durchmischung bei der Deposition von $\mathrm{Cu}$ auf $\mathrm{Ag}$ als im umgekehrten Fall. Allerdings weichen beide Verfahren in der Größe der durchmischten Bereiche stark voneinander ab. Die aus den Augerelektronenspektren bestimmten Konzentrationen zeigen einen wesentlich flacheren Verlauf, der nur im Fall der Deposition von $\mathrm{Fe}$ auf $\mathrm{Ag}$ durch die Tiefenauflösung des Spektrometers bedingt ist (siehe Kap. 3.3.1). Erst nach der Deposition von $5 \mathrm{~nm} \mathrm{Ag} \mathrm{erreicht} \mathrm{die} \mathrm{Oberflächenkonzentration} \mathrm{des} \mathrm{Ag} \mathrm{90 \% ,} \mathrm{im} \mathrm{Fall}$ von $\mathrm{Cu}$ erst nach $6 \mathrm{~nm}$. Nach den Ratenmessungen wird die 90\%-Grenze zwischen $2 \mathrm{~nm}(\mathrm{Ag}$ auf $\mathrm{Cu})$ und $3 \mathrm{~nm}(\mathrm{Cu}$ auf $\mathrm{Ag})$ erreicht, vgl. Abbildung 3-2. 


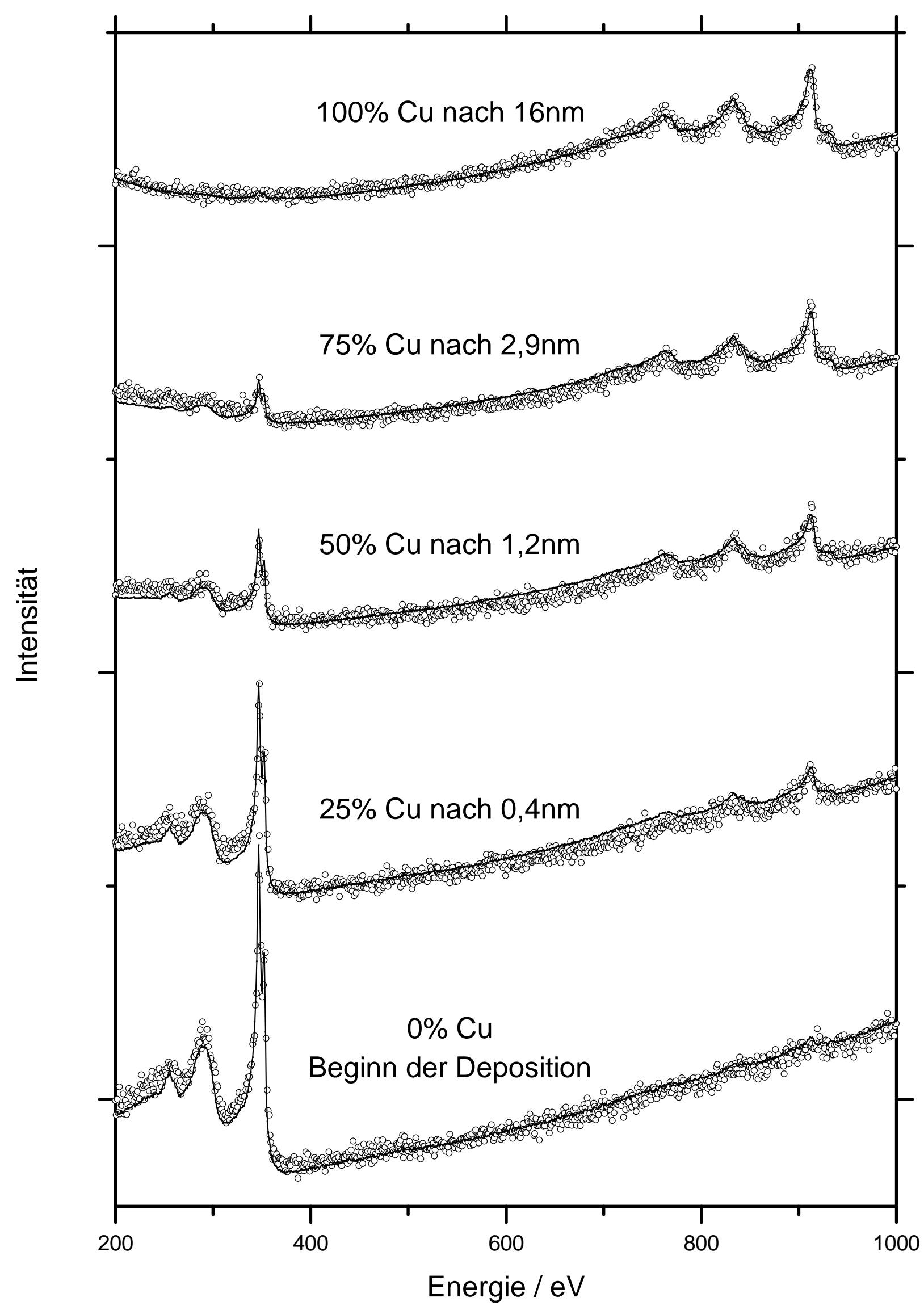

Abbildung 3-6: Augerelektronenspektren einer auf Ag wachsenden Cu-Schicht; offene Kreise: Messung; durchgezogene Linie: bester Fit (siehe Text) 


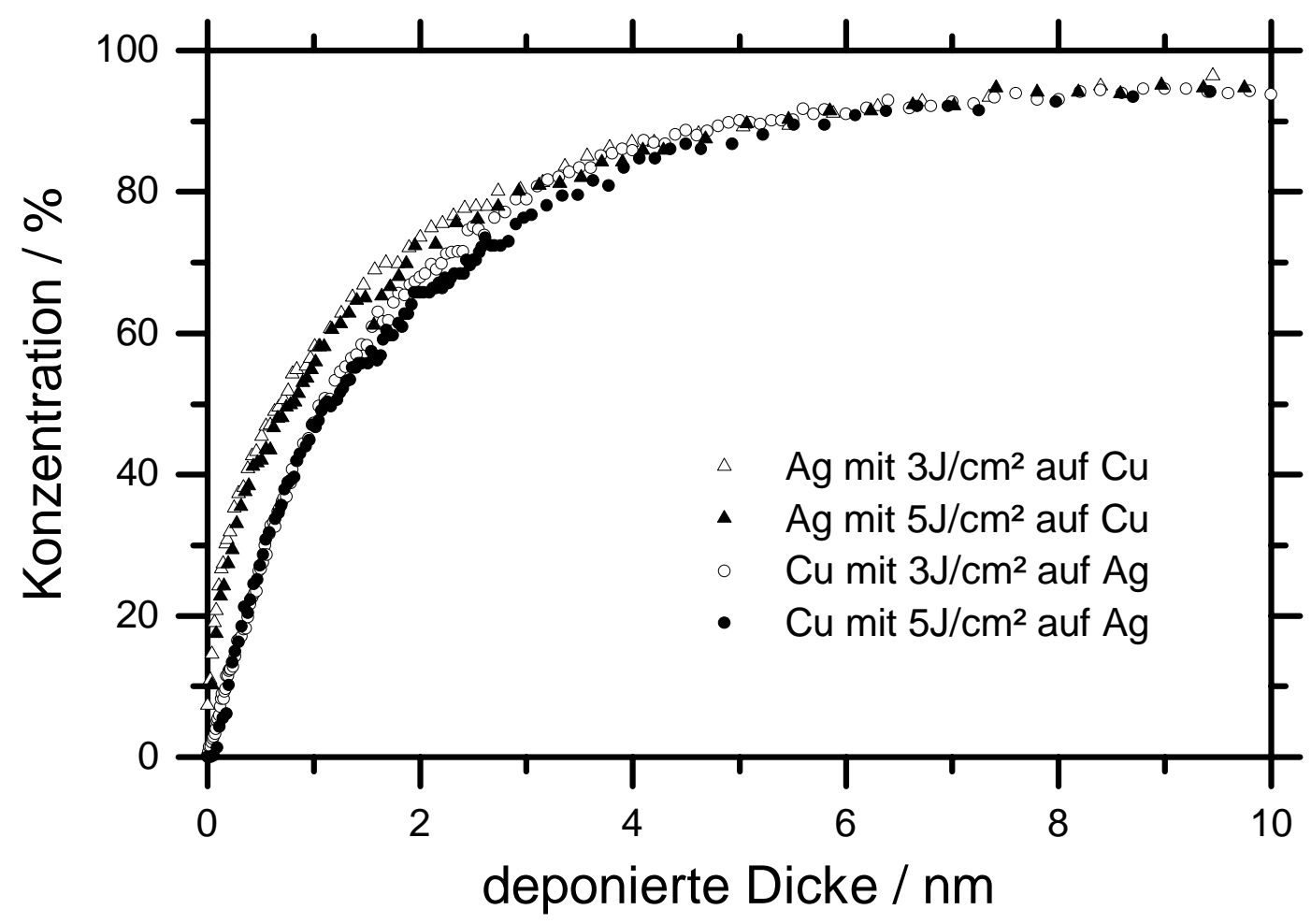

Abbildung 3-7: Konzentrationsverlauf an der Cu-Ag-Grenzfläche in Abhängigkeit von der Energiedichte am Target

Die unterschiedlichen Ergebnisse lassen sich vereinbaren, wenn der Sputteryield einer Legierung nicht linear von der Konzentration der Elemente abhängt, sondern stärker dem Sputteryield der Matrix entspricht. Eine Legierung von $\mathrm{Cu}_{90} \mathrm{Ag}_{10}$ würde sich demnach praktisch wie reines $\mathrm{Cu}$ verhalten, d. h. geringe Konzentrationen lassen sich mit der in Kap. 3.1 dargestellten Methode nicht nachweisen. Diese Argumentation setzt außerdem das Vorliegen einer homogenen Legierung im Gegensatz zu einer heterogenen Legierung und Inselwachstum voraus.

Eine Abhängigkeit des Konzentrationsverlaufes von der Laserenergiedichte auf dem Target ist in Abbildung 3-7 nicht zu erkennen, obwohl die Anzahl und Energie der Ionen des Plasmas mit der Energiedichte leicht ansteigt [21] und damit auch eine stärkere Durchmischung zu erwarten wäre.

Neben $\mathrm{Cu}-\mathrm{Ag}$ wurde die Deposition von Fe auf $\mathrm{Ag}$ und $\mathrm{Ag}$ auf Fe mittels AES untersucht. Die Ergebnisse der Konzentrationsbestimmung sind in Abbildung 3-8 dargestellt. Bei der Deposition von Ag auf Fe zeigt sich quantitativ derselbe Verlauf wie 


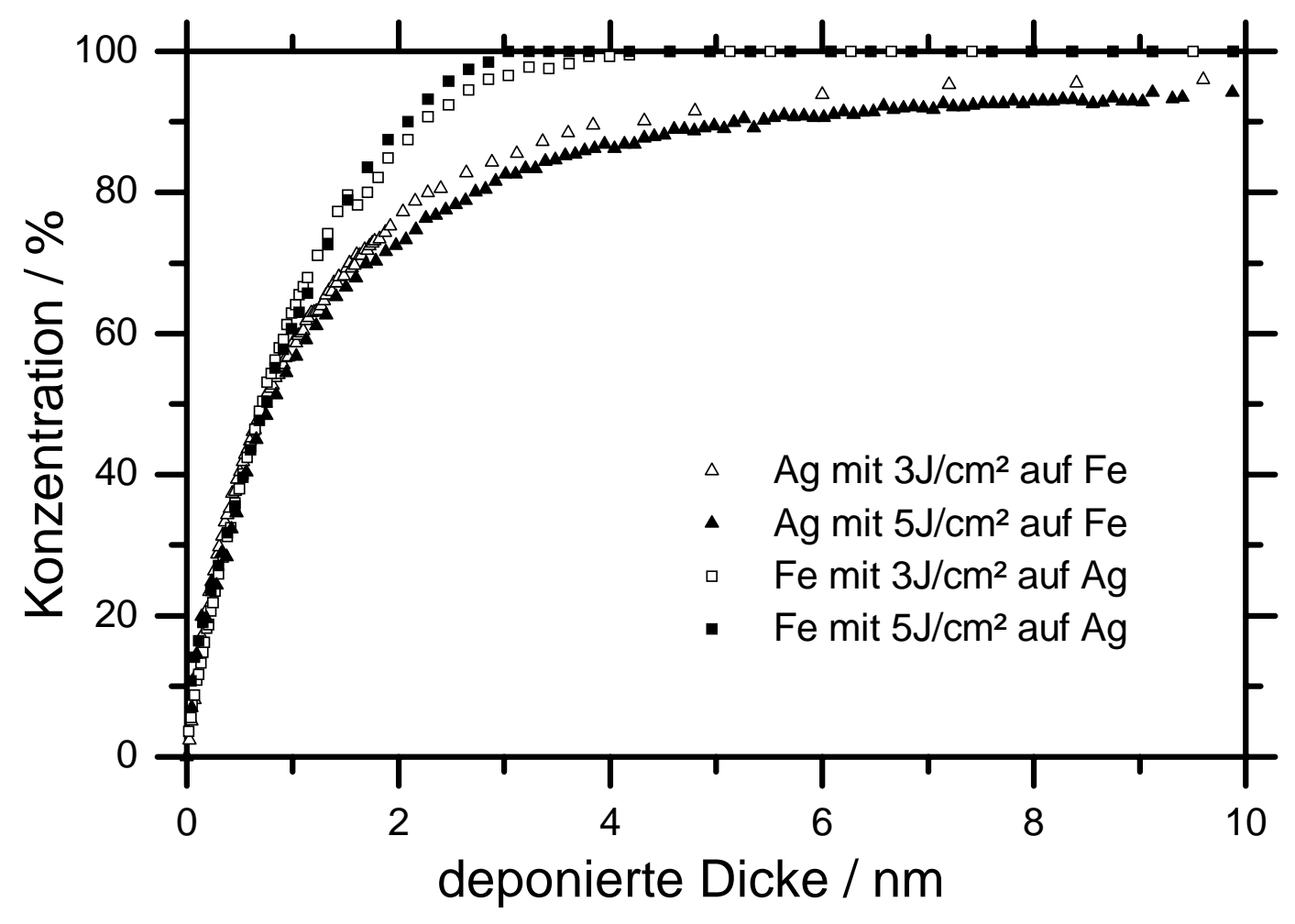

Abbildung 3-8: Konzentrationsverlauf an der Fe-Ag-Grenzfläche in Abhängigkeit von der Energiedichte am Target

bei der Deposition von Ag auf Cu. Wiederum ist im Rahmen der Meßgenauigkeit keine Abhängigkeit von der Energiedichte zu beobachten.

Bei der Deposition von Fe auf Ag wird die Ag-Oberfläche sehr schnell von Fe bedeckt (90\% Fe nach $2 \mathrm{~nm}$ ). Nach [11] wird Fe bei der PLD etwas tiefer in Ag implantiert als $\mathrm{Ag}$ in Fe, was eine stärkere Durchmischung und einen flacheren Konzentrationsverlauf bei der Deposition von Fe auf Ag erwarten läßt. Aufgrund des hohen Sputteryields von Ag (siehe Kap. 3.1) tragen die auftreffenden Fe-Atome in der Fe-AgOberflächenlegierung allerdings bevorzugt die Ag-Atome ab, was sehr schnell zu einer reinen Fe-Oberfläche führt. Dies erklärt auch, warum das in der Literatur beschriebene Aufschwimmen von Atomen einer Schicht mit niedriger Oberflächenenergie (wie z. B. $\mathrm{Ag}$ oder $\mathrm{Cu}$ ) auf eine darauf deponierte Schicht mit hoher Oberflächenenergie (z. B. Fe) $[37,38]$ hier nicht beobachtet wird. 


\subsection{Modelle zum Verlauf der Oberflächenkonzentration mit der Schichtdicke}

\subsubsection{Oberflächenkonzentration bei glatten Grenzflächen}

Da die AES eine begrenzte Tiefenauflösung besitzt, wird auch bei kompletter Bedeckung eines Materials B mit einer dünnen Schicht A ohne Grenzflächenrauhigkeit und -durchmischung (Abbildung 3-9) der gemessene Konzentrationsverlauf nicht abrupt sein. Um darzulegen, welchen Konzentrationsverlauf man in diesem Fall erwarten kann und ob dieser mit den Messungen in Einklang zu bringen ist, muß die Oberflächenkonzentration als Funktion der gemessenen Augerelektronenintensitäten ausgedrückt werden. Die Gesamtintensität der in

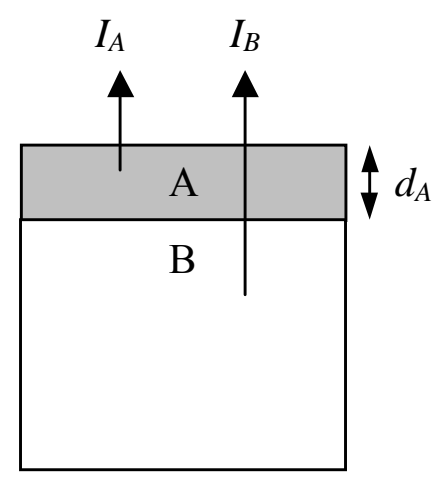
Abbildung 3-9 dargestellten Oberfläche aus

Abbildung 3-9: Schemazeichnung zur AES an einer dünnen glatten Schicht A auf Material B einer Schicht A auf einem Substrat B ist $I_{A}+I_{B}$, und die Konzentration $c_{A}$ des Elementes A ergibt sich damit zu:

$$
c_{A}=\frac{I_{A}}{I_{A}+I_{B}}
$$

Die vom Material B emittierten Augerelektronen durchqueren auf dem Weg zum Detektor die Schicht A und werden dort teilweise absorbiert, wobei die Absorption exponentiell mit der Schichtdicke $d_{A}$ der bedeckenden Schicht steigt. Die Intensität $I_{B}$ ist deshalb um einen bestimmten Faktor geringer als die Intensität des unbedeckten BMaterials $I_{B}^{0}$ :

$$
I_{B}=I_{B}^{0} \exp \left(-d_{A} / l_{A}\right)
$$

Dabei ist $l_{A}$ ist die mittlere freie Weglänge der Augerelektronen im Material A.

Die Intensität $I_{A}$ der A-Schicht ist ebenfalls geringer als die Intensität $I_{A}^{0}$ einer dicken Schicht aus A, da der Beitrag aus dem Bereich mit einer Tiefe größer als $d_{A}$ fehlt:

$$
I_{A}=I_{A}^{0}-I_{A}^{0} \exp \left(-d_{A} / l_{A}\right)
$$




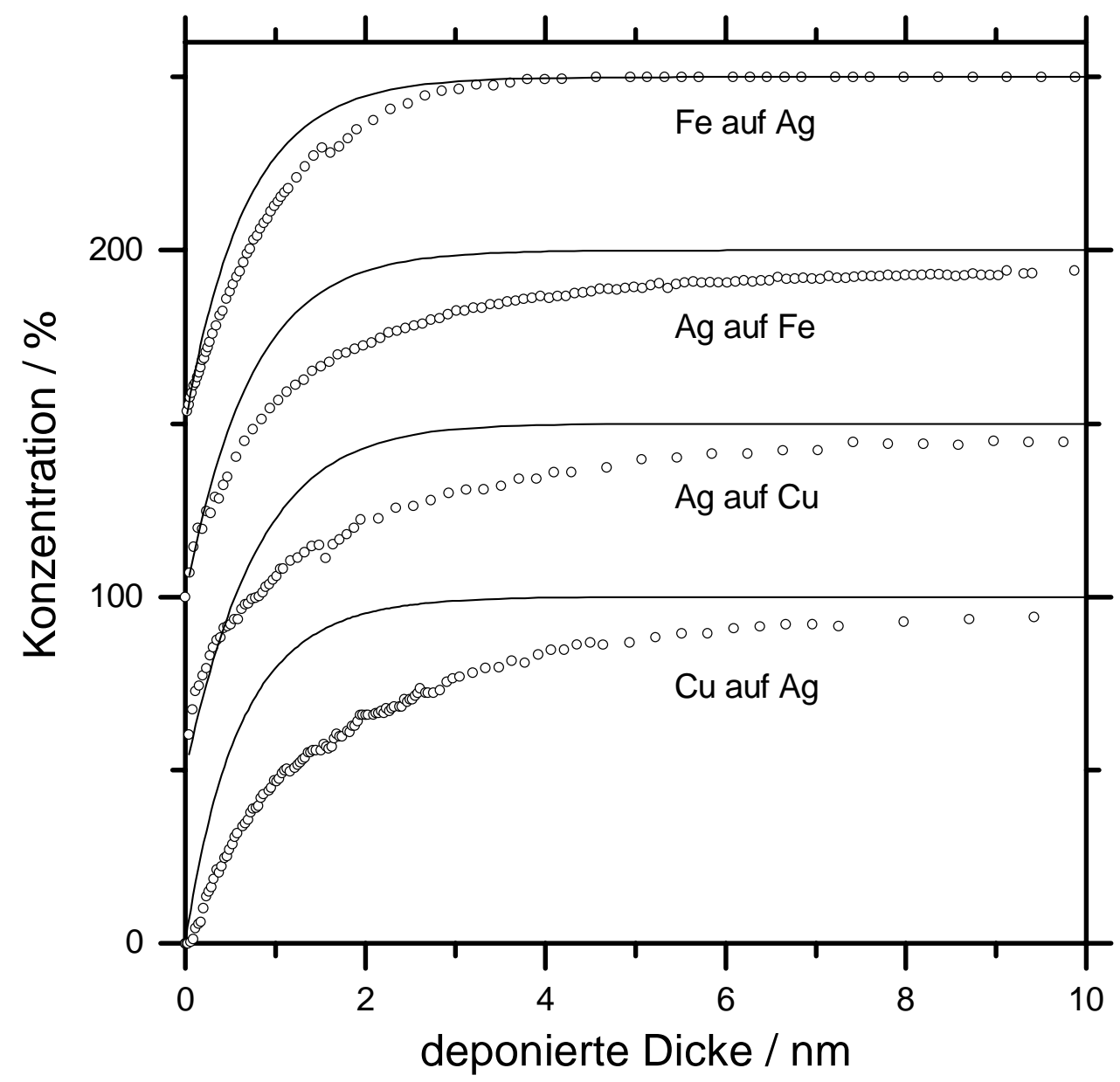

Abbildung 3-10: Oberflächenkonzentration in Abhängigkeit von der deponierten Dicke, Energiedichte $5 \mathrm{~J} / \mathrm{cm}^{2}$; Zwischen den Kurven liegt ein Versatz von jeweils $50 \%$. Die durchgezogene Linie kennzeichnet den nach (3-10) berechneten Verlauffür eine scharfe Grenzfläche.

Einsetzen von (3-8) und (3-9) in (3-7) liefert folgenden Zusammenhang:

$$
c_{A}=\left(\frac{I_{B}^{0}}{I_{A}^{0}} \frac{1}{\exp \left(d_{A} / l_{A}\right)-1}+1\right)^{-1}
$$

Bezogen auf Ag betragen die Intensitätsverhältnisse der Reinelementspektren (Abbildung 3-5) $I_{F e}^{0} / I_{A g}^{0}=1,05$ und $I_{C u}^{0} / I_{A g}^{0}=1,21$. Für die Absorptionslänge $l_{A}$ der Augerelektronen wurde entsprechend Abbildung 2-4 ein mittlerer Wert von 0,7 nm angenommen, da sich die Augerlinien der beteiligten Elemente über einen Bereich von $300 \mathrm{eV}$ bis $900 \mathrm{eV}$ erstrecken. 
Abbildung 3-10 zeigt den Vergleich der gemessenen Konzentrationsverläufe mit den aus (3-10) berechneten Kurven. Die Messungen zeigen einen deutlich flacheren Verlauf der Oberflächenkonzentration und sind somit nicht mit dem Modell einer glatten, scharfen Grenzfläche vereinbar. Nur bei der Deposition von Fe auf Ag kommt der gemessene Konzentrationsverlauf recht nahe an die berechnete Kurve heran. Der Grund für den steileren Verlauf der Oberflächenkonzentration in diesem Fall liegt in dem auf S. 33 schon erwähnten starken Absputtern der Ag-Oberfläche durch die auftreffenden Fe-Ionen und die dadurch bedingte schnelle Ausbildung einer reinen Fe-Oberfläche. Die näherungsweise Übereinstimmung mit der berechneten Kurve läßt allerdings nicht auf eine nahezu scharfe Ag-Fe-Grenzfläche schließen, denn die mittlere freie Weglänge der analysierten Elektronen von 0,7 nm gibt eine Auflösungsgrenze der AES in dieser Größe vor. Eine Durchmischung oder Rauhigkeit der Grenzfläche, die kleiner ist als dieser Wert, kann somit nicht nachgewiesen werden.

Für den flachen Konzentrationsverlauf in den anderen Fällen kann sowohl Inselwachstum als auch eine Implantation unter die Oberfläche verantwortlich sein. Für eine Implantation der bei der PLD auftretenden schnellen Ionen einige Monolagen tief in die aufwachsende Schicht gibt es mehrere Hinweise [17,39-41]. Deshalb wird im folgenden ein numerisches Modell vorgestellt, welches das Schichtwachstum unter Berücksichtigung der Implantation quantitativ beschreibt und so Aussagen über den Konzentrationsverlauf in den Grenzflächen und insbesondere die hier gemessenen Oberflächenkonzentrationen erlaubt. Der Einfluß des Inselwachstums wird unter Berücksichtigung aller Ergebnisse in Kapitel 5 diskutiert.

\subsubsection{Numerisches Modell des Schichtwachstums bei der PLD}

Das Wachstum von Schichten, die mittels PLD hergestellt wurden, unterscheidet sich von dem der weiter verbreiteten Depositionsmethoden Sputtern und Aufdampfen. Bei diesen findet das Wachstum der Schicht auf der Oberfläche statt, da die kinetische Energie der auftreffenden Teilchen $(0,1 \mathrm{eV}$ beim Aufdampfen, $10 \mathrm{eV}$ beim Sputtern [42]) nicht ausreicht, um diese in die Schicht eindringen zu lassen. Dazu wären Energien nötig, die über der Verlagerungsenergie von etwa $25 \mathrm{eV}$ pro Atom in der Schicht [43] liegen. Bei der PLD hingegen besteht der größte Teil der deponierten Teilchen aus Ionen mit einer breiten Energieverteilung, von einigen 10 bis einigen $100 \mathrm{eV}$, wobei das Maximum der Verteilung um $100 \mathrm{eV}$ liegt, je nach Material und Laserenergiedichte am Target. S. Fähler hat mittels Flugzeitmessungen die 


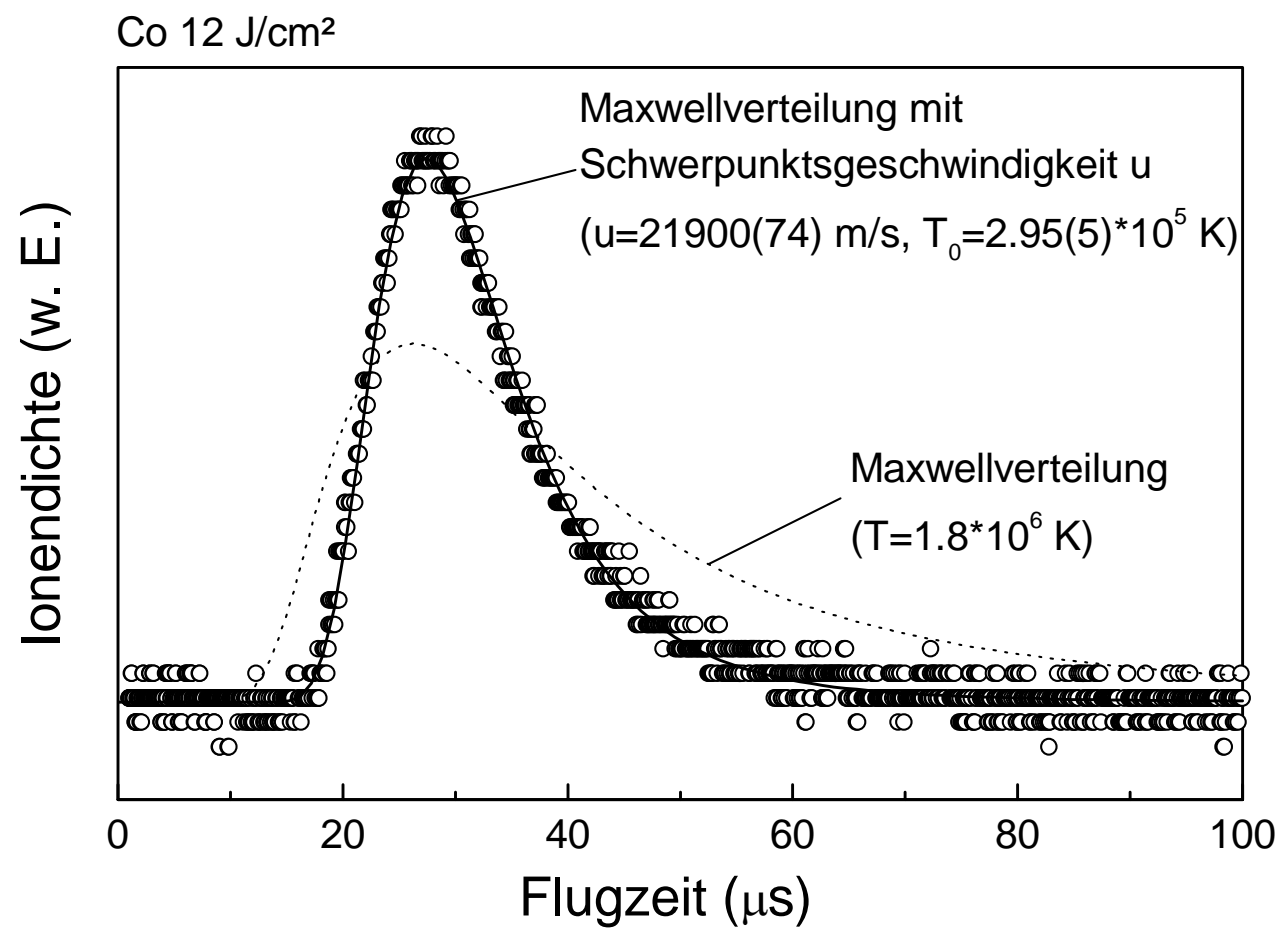

Abbildung 3-11: Geschwindigkeitsverteilung der Ionen im Plasma bei einer Laserenergiedichte von $12 \mathrm{~J} / \mathrm{cm}^{2}$ auf einem Co-Target [44]

Geschwindigkeitsverteilung der Ionen im Laserplasma für verschiedene Materialien bestimmt [21] und gezeigt, daß diese einer modifizierten Maxwellverteilung gehorcht (Abbildung 3-11):

$$
\frac{d N}{d v} \sim v^{3} \exp \left(-\frac{m\left(v-v_{0}\right)^{2}}{2 k_{B} T_{e f f}}\right)
$$

Dabei ist $d N$ die Anzahl von Ionen der Masse $m$, die sich in einem Geschwindigkeitsintervall $d v$ um die Geschwindigkeit $v$ aufhalten. Im Unterschied zur normalen Maxwellverteilung, die ebenfalls in Abbildung 3-11 dargestellt ist und mit der sich die Flugzeitmessungen nicht erklären lassen, beschreibt (3-11) die thermische Bewegung der Ionen bei einer effektiven Temperatur $T_{\text {eff, die von einer }}$ Schwerpunktsgeschwindigkeit $v_{0}$ des gesamten Plasmas überlagert ist. $k_{B}$ ist die Boltzmannkonstante.

Mit der bekannten Geschwindigkeitsverteilung der Ionen kann auf ihre Eindringtiefe in einen Festkörper geschlossen werden. Dazu wird auf ein von Betz und Wien beschriebenes Modell zurückgegriffen [44], welches die Eindringtiefe in Abhängigkeit von der Energie der auftreffenden Ionen sowie der Ordnungszahl und Atommasse der 
Ionen und des Festkörpers beschreibt. Dieses Modell wurde schon in [11] erfolgreich zur Beschreibung der Leitfähigkeit laserdeponierter Schichtpakete eingesetzt.

Beim Auftreffen eines energetischen Teilchens auf einen Festkörper wird es durch Coulomb-Wechselwirkung mit den Atomkernen und den Hüllenelektronen abgebremst. Für Teilchenenergien im keV-Bereich und darunter - also für die bei der Laserdeposition auftretenden Energien - ist nur der Energieverlust durch Streuung an den Atomkernen von Bedeutung. Die Stärke der Streuung wird durch den Energieverlust $d E / d x$ des Teilchens pro Wegstrecke dargestellt und kann aus dem abgeschirmten Coulomb-Potential in einer Entfernung $r$ von einer Punktladung abgeleitet werden, welches folgende Form hat:

$$
V(r)=\frac{Z_{1} Z_{2} e^{2}}{r} \Phi\left(\frac{r}{a}\right)
$$

$Z_{1}$ und $Z_{2}$ sind die Ordnungszahlen der Stoßpartner, $e$ die Elementarladung und $\Phi$ eine exponentiell gegen Null gehende Funktion, die die teilweise Abschirmung der Kernladung durch das umgebende Elektronengas beschreibt. Für das hier verwendete Krypton-Kohlenstoff-Potential [44] hat man empirisch folgende Gleichung gefunden:

$$
\Phi(x)=0,190945 e^{-0,131825 x}+0,473674 e^{-0,63717 x}+0,335381 e^{-1,919249 x}
$$

$x=r / a$ wird in Einheiten des Lindhardschen Abschirmradius $a$ angegeben:

$$
a=\frac{0,8854 a_{0}}{\sqrt{Z_{1}^{2 / 3}+Z_{2}^{2 / 3}}}
$$

wobei $a_{0}=0,0529 \mathrm{~nm}$ der Bohr-Radius ist.

Der aus dieser Form des Potentials folgende Wirkungsquerschnitt $s_{n}(\varepsilon)$ für Kernstreuung kann unabhängig von der verwendeten Kombination Ion/Festkörper näherungsweise analytisch ausgedrückt werden:

$$
\begin{aligned}
s_{n}(\varepsilon) & =\frac{0,5 \ln (1+\varepsilon)}{\varepsilon+0,10718 \varepsilon^{0,37544}} \\
\varepsilon & =\frac{M_{2}}{M_{1}+M_{2}} \frac{a}{Z_{1} Z_{2} e^{2}} E
\end{aligned}
$$

$\varepsilon$ ist eine dimensionslose Energie, die sich über Masse und Ordnungszahl des auftreffenden Teilchens $\left(M_{1}, Z_{1}\right)$ und der Atome des Festkörpers $\left(M_{2}, Z_{2}\right)$ aus der 
kinetischen Energie $E$ des Teilchens ergibt. Aus $s_{n}(\varepsilon)$ bestimmt sich der Energieverlust pro Wegstrecke zu

$$
\frac{d E}{d x}=509,6 \frac{\rho Z_{1} Z_{2} M_{1}}{M_{2}\left(M_{1}+M_{2}\right) \sqrt{Z_{1}^{2 / 3}+Z_{2}^{2 / 3}}} s_{n}(\varepsilon)
$$

Hier geht neben den Ordnungszahlen $Z_{1}, Z_{2}$ und Massen $M_{1}, M_{2}$ auch die Dichte $\rho$ des Festkörpers ein. Der Vorfaktor ist so gewählt, daß sich $\mathrm{eV} / \mathrm{nm}$ als Einheit für $d E / d x$ ergibt, wenn $\rho$ in $\mathrm{g} / \mathrm{cm}^{3}$ angegeben wird.

Ein sich im Festkörper über eine Strecke $d x$ bewegendes Teilchen verliert entsprechend (3-17) den Betrag $d E$ an kinetischer Energie. Um die Gesamtstrecke $R\left(E_{0}\right)$ zu berechnen, die das Teilchen bis zum Stillstand zurücklegt, integriert man von der Auftreffenergie $E_{0}$ bis 0 :

$$
R\left(E_{0}\right)=-\int_{E_{0}}^{0} \frac{d E}{(d E / d x)}
$$

Da das einfallende Teilchen bei der Abbremsung im Target in seiner Richtung abgelenkt werden kann, entspricht die zurückgelegte Wegstrecke $R$ allerdings selbst bei senkrechtem Einfall nicht genau der Tiefe, bis zu der das Teilchen vorstößt. Es ergibt sich statt dessen eine Verteilung $\Delta R_{P}$ von Eindringtiefen um eine mittlere Tiefe $R_{P}$ (,projected range“). Näherungsweise gilt [45]:

$$
\begin{gathered}
\frac{R}{R_{P}}=1+\frac{1}{3} \frac{M_{2}}{M_{1}} \\
\frac{\left(\Delta R_{P}\right)^{2}}{R_{P}{ }^{2}}=\frac{2}{3} \frac{M_{1} M_{2}}{\left(M_{1}+M_{2}\right)^{2}}
\end{gathered}
$$

Bis hierher wurde nur Implantation in Festkörper aus reinen Elementen betrachtet. Um Implantation in eine homogene Legierung korrekt zu beschreiben, müssen die Beiträge der Legierungspartner 1 und 2 zum Streuquerschnitt nach ihrer Volumenkonzentration $c_{1}, c_{2}$ gewichtet werden. Die Wichtung erfolgt über die Dichte $\rho_{1}, \rho_{2}$ der Elemente:

$$
\frac{d E}{d x}=\left(\frac{d E}{d x}\right)_{1}+\left(\frac{d E}{d x}\right)_{2}=\left(\frac{d E}{d x}\right)\left(c_{1} \rho_{1}\right)+\left(\frac{d E}{d x}\right)\left(c_{2} \rho_{2}\right)
$$

Wird die Berechnung der Implantationstiefe für alle aus der Geschwindigkeitsverteilung (3-11) bestimmten Auftreffenergien durchgeführt, ergibt sich eine von der Tiefe abhängige Konzentration der implantierten Teilchen. Abbildung 3-12 zeigt das 


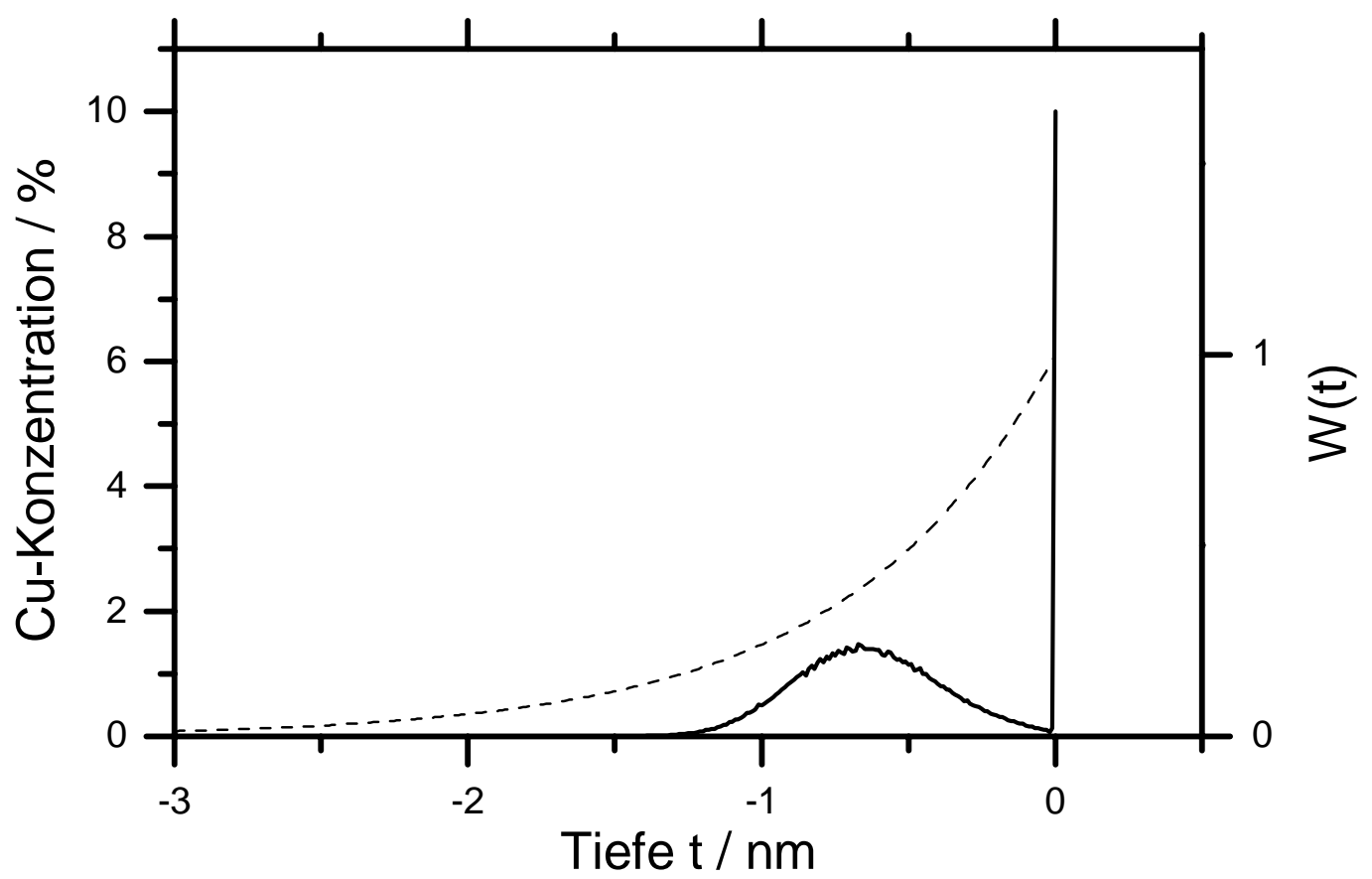

Abbildung 3-12: Implantationsprofil nach der Deposition von 0,01 $\mathrm{nm} \mathrm{Cu}$ auf eine dicke Ag-Schicht; gestrichelte Linie: Verlauf der Wichtungsfunktion W(t) für die Bestimmung der Oberflächenkonzentration

Konzentrationsprofil, nachdem ein Puls $\mathrm{Cu}$ auf eine dicke Ag-Schicht deponiert wurde. Es wurde eine Depositionsrate von $0,01 \mathrm{~nm} / \mathrm{Puls}$ und der Ionenanteil mit $90 \%$ angenommen. Für $T_{\text {eff }}$ und $v_{0}$ der Ionen wurden die in [21] bestimmten Werte von $2,4 \cdot 10^{5} \mathrm{~K}$ und $1,5 \cdot 10^{4} \mathrm{~m} / \mathrm{s}$ verwendet. Es ist $\mathrm{zu}$ erkennen, wie die Ionen bis $\mathrm{zu}$ etwa $1 \mathrm{~nm}$ tief unter die Oberfläche der Ag-Schicht implantiert werden. Die breite Tiefenverteilung wird sowohl durch die Geschwindigkeitsverteilung der Ionen im Plasma (charakterisiert durch $T_{e f f}$ ) als auch die ballistische Streuung in der Ag-Schicht nach (3-20) hervorgerufen.

In [46] wurde durch spektroskopische Flugzeitmessungen an den Neutralteilchen der Plasmawolke ihre mittlere kinetische Energie zu $10 \mathrm{eV}$ bestimmt, selbst bei einer sehr hohen Energiedichte von $17 \mathrm{~J} / \mathrm{cm}^{2}$. Damit werden sie nicht implantiert, sondern direkt auf der Oberfläche deponiert, was zu dem in Abbildung 3-12 dargestellten steilen Anstieg der $\mathrm{Cu}$-Konzentration führt. Daß unter dem angenommenen Atomanteil von $10 \%$ die Konzentration an der Oberfläche auf 10\% steigt, ist durch die Dickenauflösung des Modells bestimmt, welche in diesem Fall mit 0,01 nm gleich der Depositionsrate pro Puls ist. 


\subsubsection{Simulation der Oberflächenkonzentration}

Für die über die AES bestimmte Oberflächenkonzentration erstreckt sich die „Oberfläche“ allerdings über einen viel tieferen Bereich von einigen Monolagen. Um aus dem simulierten Konzentrationsprofil eine zur AES analoge Konzentration zu berechnen, wurden die Konzentrationswerte ausgehend von der Oberfläche mit einem exponentiell abfallenden Faktor gewichtet. Dies entspricht dem Verlauf der Augerelektronenintensität mit der Tiefe, aus der die Elektronen emittiert werden.

Die Stärke des Abfalls ist abhängig von der kinetischen Energie der Elektronen (vgl. Abbildung 2-4) und dem absorbierenden Material, welches sie auf dem Weg zur Oberfläche durchqueren. $\mathrm{Da}$ sich die für die Konzentrationsbestimmung herangezogenen Augerlinien über einen Bereich von $300 \mathrm{eV}$ bis $900 \mathrm{eV}$ erstrecken und die Konzentration der Elemente in der Schicht variiert, wurde eine konstante mittlere Informationstiefe $t_{0}=0,7 \mathrm{~nm}$ angesetzt. Für den Verlauf des Wichtungsfaktors $W$ mit der von der Oberfläche aus gemessenen Tiefe $t$ ergibt sich somit:

$$
W=e^{-t / t_{0}}
$$

Dieser Verlauf ist in Abbildung 3-12 als gestrichelte Linie dem Konzentrationsprofil überlagert. Die Oberflächenkonzentration $c_{O b}$ berechnet sich damit zu:

$$
c_{O b}=\frac{\int_{0}^{\infty} c(t) \cdot e^{-t / t_{0}} d t}{\int_{0}^{\infty} e^{-t / t_{0}} d t}=\frac{1}{t_{0}} \int_{0}^{\infty} c(t) \cdot e^{-t / t_{0}} d t
$$

Dabei ist $c(t)$ die in der Tiefe $t$ herrschende Konzentration des betrachteten Elementes. In der numerischen Umsetzung von (3-23) kann die Integration nicht bis unendlich geführt werden. Sie wird statt dessen abgebrochen, wenn der Wichtungsfaktor unter $10^{-3}$ fällt.

In Abbildung 3-12 ist klar zu erkennen, daß neben den Atomen auf der Oberfläche auch die implantierten Ionen einen nicht unwesentlichen Beitrag zur Oberflächenkonzentration liefern. Der nach jedem Puls mittels (3-23) berechnete Wert für die Oberflächenkonzentration hängt somit neben dem Ionenanteil im Plasma auch von der Schwerpunktsgeschwindigkeit des Plasmas $v_{0}$, welche die mittlere Implantationstiefe bestimmt, und von der Plasmatemperatur $T_{\text {eff }} \mathrm{ab}$, die eine Verbreiterung der Verteilung der implantierten Ionen bewirkt. Abbildung 3-13 zeigt, 
wie sich eine Variation dieser Parameter auf den Verlauf der Oberflächenkonzentration auswirkt. In Abbildung 3-13a ist zu erkennen, daß mit der Erhöhung von $v_{0}$ die Konzentration wie erwartet flacher verläuft. Der Ionisierungsgrad wurde hier mit 100\% angenommen und $T_{\text {eff }}$ bei $160000 \mathrm{~K}$ konstant gehalten. Bei geringen Geschwindigkeiten unter $5 \mathrm{~km} / \mathrm{s}$ unterscheiden sich die Konzentrationsverläufe nicht mehr, da dann die Implantationstiefe kleiner als die durch die Plasmatemperatur bestimmte Breite der Verteilung wird.

In Abbildung 3-13b ist dargestellt, wie sich $T_{\text {eff }}$ bei einer konstanten Plasmageschwindigkeit von $8,8 \mathrm{~km} / \mathrm{s}$ auswirkt. Anders als im Fall a hat hier eine Erhöhung der Temperatur einen steileren Verlauf der Oberflächenkonzentration zur Folge. Dieses zunächst widersprüchlich erscheinende Verhalten läßt sich über Geschwindigkeitsverteilung der Ionen im Plasma nach (3-11) begründen: Da diese Gleichung eine thermische Bewegung um eine Schwerpunktsgeschwindigkeit herum beschreibt, führt eine Erhöhung von $T_{\text {eff }}$ dazu, daß sowohl schnellere als auch langsamere Ionen auf die Oberfläche der Schicht auftreffen. Damit wird das Implantationsprofil sowohl in größere Tiefen als auch in Richtung Oberfläche ausgedehnt. Aufgrund der abnehmenden Empfindlichkeit der AES mit der Tiefe (Abbildung 3-12) gehen hauptsächlich die näher der Oberfläche implantierten Ionen in die Berechnung ein, was den beobachteten schnelleren Anstieg der Konzentration zur Folge hat. ${ }^{4}$ Bei Temperaturen über $2 \cdot 10^{5} \mathrm{~K}$ wird eine Sättigung erreicht und die Konzentrationen zeigen den gleichen Verlauf.

Der Einfluß des Ionenanteils ist in Abbildung 3-13c dargestellt. Es wird ein flacherer Konzentrationsverlauf mit steigendem Anteil an Ionen beobachtet. Bis zu relativ hohen Ionenanteilen wird die Kurve hauptsächlich durch die auf der Oberfläche deponierten Atome bestimmt und ist vergleichsweise steil. Eine merkliche Abflachung tritt erst über einem Ionenanteil von $80 \%$ ein.

\footnotetext{
${ }^{4}$ Der nach erfolgter Deposition berechnete Konzentrationsverlauf über die Grenzfläche wird dagegen erwartungsgemäß flacher mit steigender Plasmatemperatur. Hier wird deutlich, daß der Verlauf der Oberflächenkonzentration nicht mit der nach der Deposition vorliegenden Konzentration in der Grenzfläche gleichgesetzt werden kann.
} 
a)

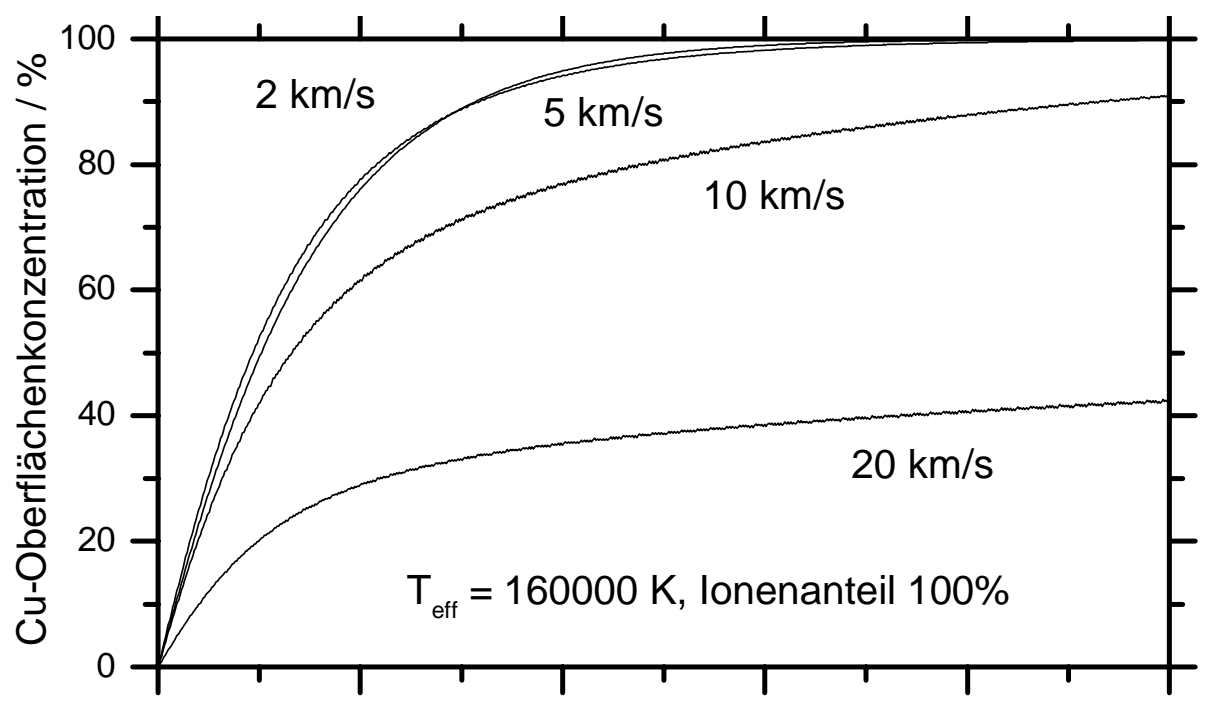

b)
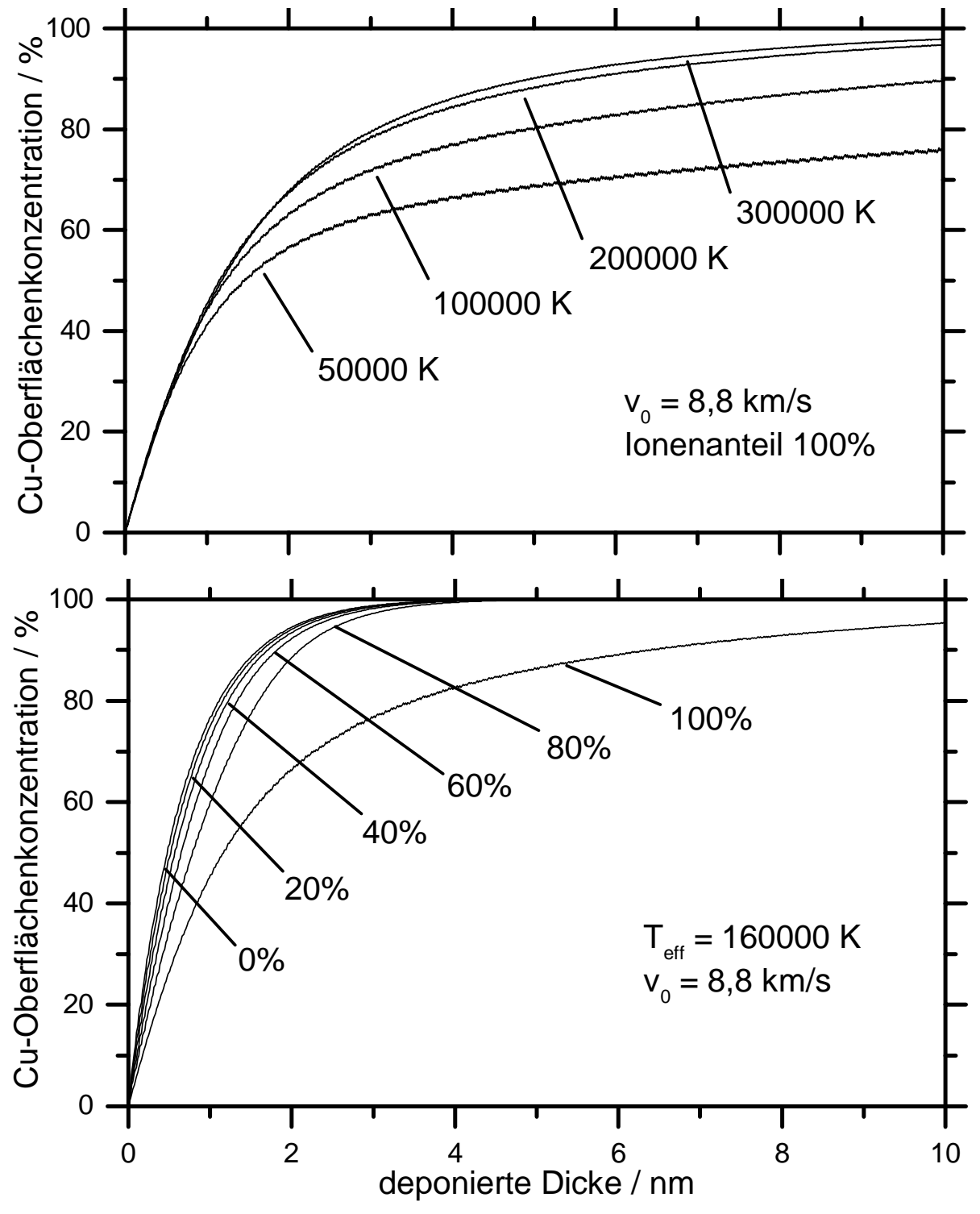

Abbildung 3-13: Einfluß der Simulationsparameter auf den Verlauf der Oberflächenkonzentration bei der Deposition von Cu auf Ag; a) Variation der Plasmageschwindigkeit $v_{0}$, b) Variation der Plasmatemperatur $T_{\text {eff, }}$ c) Variation des Ionenanteils 
Die hier beschriebenen Simulationsparameter wurden in physikalisch sinnvollen Grenzen variiert, bis die beste Übereinstimmung mit den gemessenen Konzentrationsverläufen erzielt wurde. Dabei sind diese Parameter nicht völlig frei, sondern aus früheren Messungen mehr oder weniger gut bekannt. Plasmatemperatur und -geschwindigkeit wurden beispielsweise in [21] in Abhängigkeit von der Laserenergiedichte auf dem Target für verschiedene Elemente bestimmt. Allerdings sind die Werte für die Energiedichte nicht direkt übertragbar, da im vorliegenden Fall mit einer räumlich konstanten Energiedichte im Gegensatz zu einer gaußförmigen Verteilung in [21] gearbeitet wurde.

Für den Ionenanteil im Laserplasma werden in der Literatur sehr unterschiedliche Werte angegeben. Diese reichen von nahe Null [47] über einige Prozent [48] bis zu hohen Anteilen [49,50] und vollständiger Ionisation [51]. Da der Ionenanteil offensichtlich stark von den herrschenden Ablationsbedingungen abhängt, werden die in [11,21,22] unter vergleichbaren Bedingungen ermittelten Werte von 50 bis 100\% zum Vergleich herangezogen.

Abbildung 3-14 zeigt die gemessenen und die simulierten Oberflächenkonzentrationen in Abhängigkeit von der deponierten Schichtdicke für die untersuchten Elementkombinationen. Wie schon in Kapitel 3.3.1 werden hier repräsentativ die bei einer Laserenergiedichte von $5 \mathrm{~J} / \mathrm{cm}^{2}$ aufgenommenen Daten ausgewertet, da diese mit den Messungen bei $3 \mathrm{~J} / \mathrm{cm}^{2}$ praktisch identisch sind.

Für den Fall der Deposition von Fe auf Ag ergibt sich nur dann eine gute Übereinstimmung mit dem gemessenen Kurvenverlauf, wenn reine Atomdeposition und eine mittlere Informationstiefe $t_{0}=0,9 \mathrm{~nm}$ angenommen wird (gestrichelte Kurve in Abbildung 3-14). Dieser Wert ist etwas höher als der in Kapitel 3.3.1 bestimmte von 0,7 nm, aber noch mit den in Abbildung 2-4 dargestellten Messungen verträglich. Die Annahme einer reinen Atomdeposition steht allerdings im Widerspruch zu früheren Messungen [11,22], die einen Ionenanteil über $80 \%$ und eine merkliche Implantation erwarten lassen. Wird die Simulation mit 80\% Ionenanteil und bei der Energiedichte von $5 \mathrm{~J} / \mathrm{cm}^{2} \mathrm{zu}$ erwartenden Plasmaparametern durchgeführt, erhält man die durchgezogene Kurve in Abbildung 3-14. Diese weicht deutlich von den Meßdaten ab. Erklären läßt sich diese Abweichung durch das starke Sputtern der Ag-Atome durch die auftreffenden Fe-Ionen. Dies führt, wie schon in Kapitel 3.2.2 erläutert, zu einem Abtrag der Ag-Oberfläche, wodurch zusätzlich zu den auf der Oberfläche deponierten 


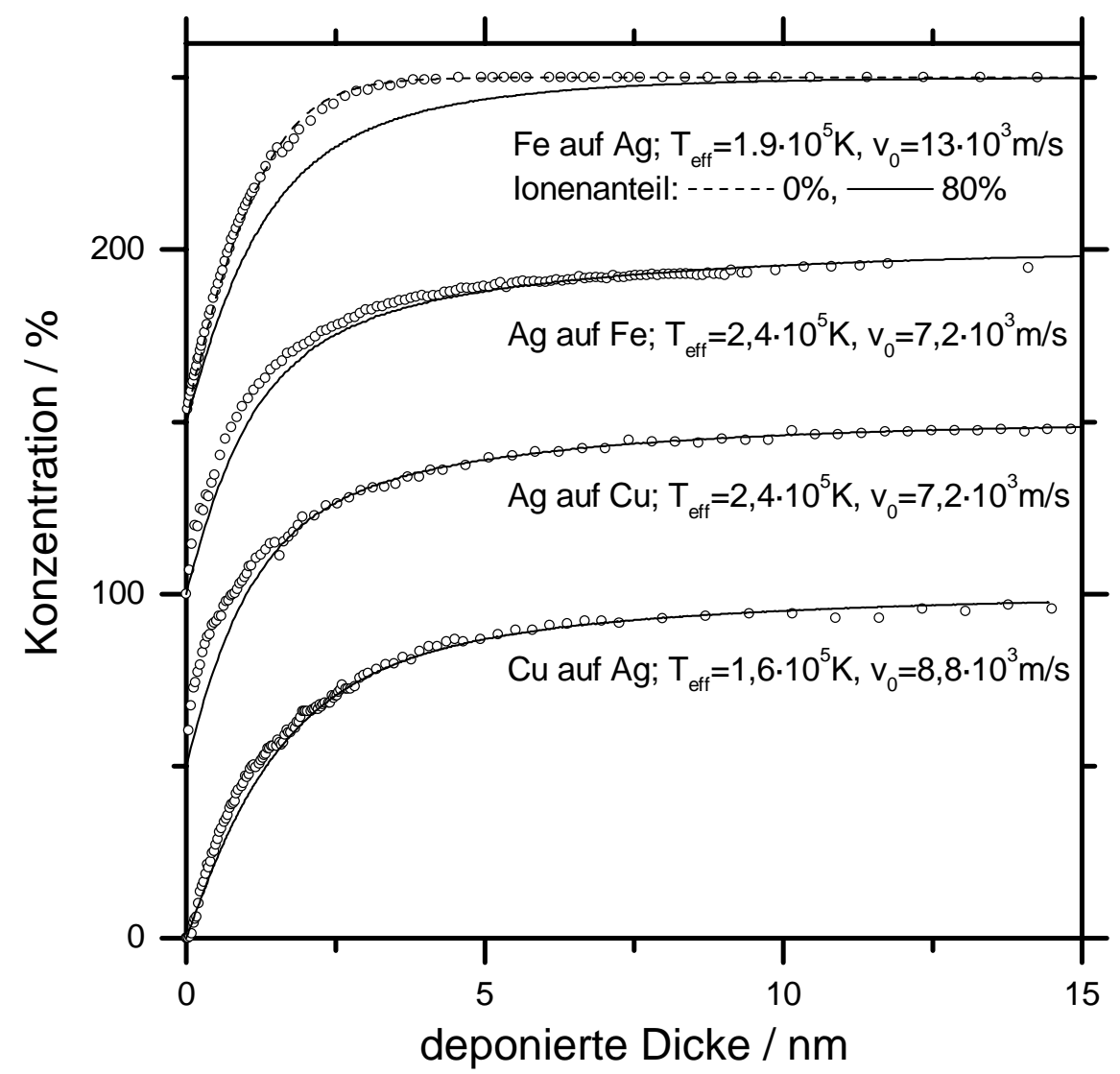

Abbildung 3-14: Vergleich der simulierten Oberflächenkonzentration (durchgezogene Linien) mit den Messungen (offene Kreise); Es wurde reine Ionendeposition simuliert (außer bei Fe auf Ag). Bei reiner Atomdeposition im Fall von Fe auf Ag erhält man die gestrichelte, mit einem Ionenanteil von $80 \%$ die durchgezogene Kurve. Für die angegebenen Parameter ergibt sich die dargestellte beste Übereinstimmung.

Atomen die implantierten Fe-Atome freigelegt werden ${ }^{5}$, welche aufgrund ihres geringen Eigensputteryields kaum abgetragen werden und eine reine Fe-Oberfläche ausbilden.

Im umgekehrten Fall der Deposition von Ag auf Fe wird ein flacherer Verlauf der Oberflächenkonzentration beobachtet, der gut mit der berechneten Kurve verträglich ist. Die Plasmaparameter $T_{\text {eff }}$ und $v_{0}$, die als freie Parameter in die Simulation eingehen, sind bei der gezeigten besten Anpassung an die Meßdaten um etwa 40\% gegenüber den bei einer Laserenergiedichte von $8 \mathrm{~J} / \mathrm{cm}^{2}$ bestimmten Werten [21] verringert, was aufgrund der hier verwendeten geringeren Energiedichte von $5 \mathrm{~J} / \mathrm{cm}^{2} \mathrm{zu}$ erwarten ist.

\footnotetext{
${ }^{5} \mathrm{Da}$ die Ag-Oberfläche bis zu den implantierten Fe-Atomen abgetragen werden muß, zeigt der Verlauf der Tiefensensitivität der AES in Abbildung 3-12. Diese zeigt, daß bis zur Tiefe der implantierten Atome ein merklicher Beitrag zur Oberflächenkonzentration geleistet wird.
} 
Abweichungen $\mathrm{zu}$ früheren Messungen treten bei dem ebenfalls als Parameter in die Simulation eingehenden Anteil der Ionen im Laserplasma auf. In [22] wurde der Ionenanteil bei $\mathrm{Ag}$-Deposition mit $5 \mathrm{~J} / \mathrm{cm}^{2} \mathrm{zu} 62 \%$ bestimmt. Die Simulation zeigt dagegen die beste Übereinstimmung mit den Messungen, wenn ein Ionenanteil von 95 bis $100 \%$ vorausgesetzt wird und damit praktisch keine Atome auf der Oberfläche deponiert werden. Diese Diskrepanz in den Ionenanteilen läßt sich erklären, wenn man annimmt, daß selbst bei einem relativ hohen Anteil von 38\% auf die Oberfläche auftreffenden Atomen diese nicht liegenbleiben, sondern aufgrund des hohen Eigensputteryields des $\mathrm{Ag}$ von schnellen Ionen des nächsten Laserpulses wieder abgetragen werden.

Zwischen $\mathrm{Cu}$ und $\mathrm{Ag}$ besteht kein so starker Gegensatz in den Sputtereigenschaften, so daß die Messungen weniger vom präferentiellen Sputtern einer Komponente beeinflußt werden und eine bessere Übereinstimmung mit den Simulationsrechnungen erzielt wird. Für die Simulation der Deposition von $\mathrm{Ag}$ auf $\mathrm{Cu}$ wurden dieselben Parameter verwendet wie im Fall von Ag auf Fe, da das Laserplasma in beiden Fällen das selbe ist. Bei $\mathrm{Cu}$-Deposition auf $\mathrm{Ag}$ sind $T_{\text {eff }}$ und $v_{0}$ des $\mathrm{Cu}$-Plasmas ebenfalls um etwa $40 \%$ gegenüber den in [21] bei $8 \mathrm{~J} / \mathrm{cm}^{2}$ bestimmten Werten verringert.

Wie im Fall der Deposition von $\mathrm{Ag}$ auf $\mathrm{Fe}$ ist auch bei $\mathrm{Cu}$ und $\mathrm{Ag}$ die beste Übereinstimmung zwischen Messung und Simulation zu erzielen, wenn ein Ionenanteil von $100 \%$ vorausgesetzt wird. Hier läßt sich der Unterschied zu den Messungen aus [22] (72\% Ionenanteil bei Cu-Deposition) nicht über einen stark unterschiedlichen Sputteryield zwischen $\mathrm{Cu}$ und $\mathrm{Ag}$ erklären. Um zu zeigen, daß auch in diesem Fall bei einem relativ hohen Anteil direkt auf der Oberfläche deponierter Atome diese dort schnell wieder abgetragen werden, kann man wie folgt argumentieren: Die bei einem einzelnen Laserpuls auf der Oberfläche deponierten Atome sind als isoliert liegende Fremdatome nur schwach an die Atome der Schicht gebunden $(\mathrm{Cu}-\mathrm{Ag}$ ist ein entmischendes System) und lassen sich darum auch leichter wieder abtragen. Damit kann der Eigensputteryield eines Elementes nicht mehr als konstant angenommen werden, sondern ist von der Konzentration abhängig, in der das Element in einer Oberflächenlegierung vorliegt.

Der in Abbildung 3-14 dargestellte Verlauf der Oberflächenkonzentration ist nicht identisch mit dem Konzentrationsverlauf über die Grenzfläche nach erfolgter Deposition. Dieser kann mit den hier beschriebenen in-situ-Verfahren nicht direkt 


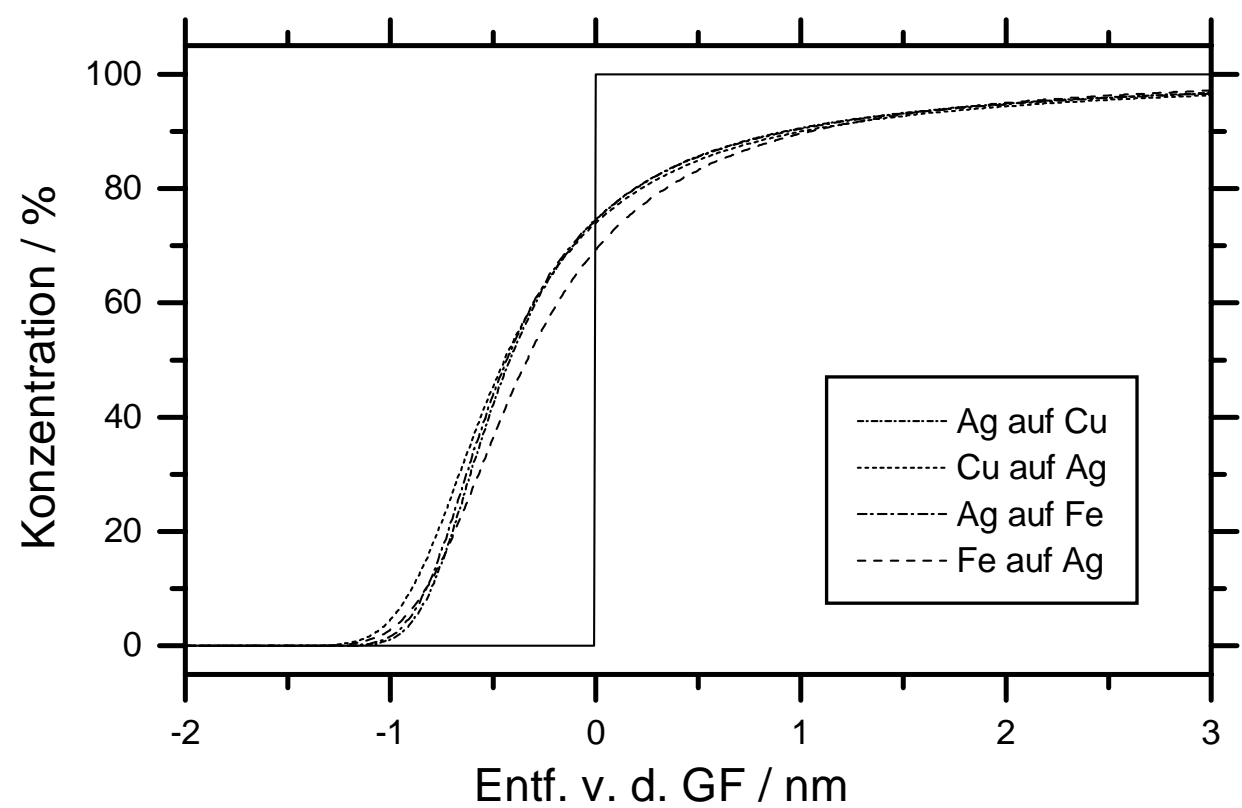

Abbildung 3-15: Konzentrationsverlauf über die Grenzfläche für die in Abbildung 3-14 dargestellten simulierten Doppelschichten; Die durchgezogene Linie kennzeichnet den Verlauf für eine scharfe Grenzfläche bzw. den Ort der ursprünglichen Oberfläche des Substratelements.

bestimmt werden. Allerdings läßt sich aus den simulierten Doppelschichten ein solches Konzentrationsprofil extrahieren.

Die Grenzflächen-Konzentrationsprofile der mit den in Abbildung 3-14 angegebenen Parametern simulierten Doppelschichten sind in Abbildung 3-15 aufgetragen. Dabei wurde die ursprüngliche Oberfläche der unteren Schicht als Nullpunkt der X-Achse angesetzt. Im Unterschied $\mathrm{zu}$ der in Abbildung 3-14 dargestellten Oberflächenkonzentration ist hier ein in etwa s-förmiger Verlauf zu erkennen, wenn auch mit stark asymmetrischem Profil. Der Anstieg der Konzentration ist an der dem Substrat zugewandten Seite steil und flacht auf der anderen Seite immer mehr ab, so daß mehrere Nanometer in die deponierte Schicht hinein die Konzentration der darunterliegenden Schicht noch einige Prozent beträgt. Die Breite der Grenzfläche ist insgesamt aber geringer, als es der Verlauf der Oberflächenkonzentration in Abbildung 3-14 vermuten läßt. 90\% der Konzentrationsänderung erstrecken sich über einen Bereich von $2 \mathrm{~nm}$. Einzige Ausnahme ist die durch die Deposition von Fe auf Ag gebildete Grenzfläche. Im Rahmen der Auflösung der AES von 0,7 nm ist diese als scharf anzusehen. Eine Simulation mit realistischen Plasmaparametern führt jedoch zu einem Verlauf über die Grenzfläche (gestrichelte Linie in Abbildung 3-15), die 
vergleichbar mit den anderen Elementkombinationen ist. Möglicherweise tritt in diesem Fall wegen der hohen positiven Mischungsenthalpie des Fe-Ag-Systems schon während der Deposition eine Segregation der implantierten Fe-Atome an die Oberfläche ein, so daß sich das durch die Implantation ausgebildete Konzentrationsprofil in eine scharfe Grenzfläche (d.h. $<0,7 \mathrm{~nm})$ umwandelt.

Auch im Fall der Deposition von Ag auf Fe ist mit einer teilweisen Entmischung in der Grenzfläche zu rechen - nach [52] lassen sich homogene Fe-Ag-Legierungen nur bis zu einer Konzentration von $15 \%$ Ag im $\mathrm{Fe}$ herstellen. Ein kontinuierlicher Konzentrationsverlauf ist daher nur auf der Fe-reichen und der Ag-reichen Seite bis zur kritischen Konzentrationen für homogene Legierungen $\mathrm{zu}$ erwarten, im mittleren Konzentrationsbereich ist mit einer heterogenen Legierung zu rechnen.

Für das System Cu-Ag mit seiner deutlich kleineren Mischungsenthalpie von 4,8 kJ/mol [14] sind keine Entmischungsvorgänge in den Grenzflächen zu erwarten, da sich die Legierung im gesamten Konzentrationsbereich homogen übersättigen läßt und bei Raumtemperatur stabil bleibt $[15,53]$. Erst bei Temperaturen oberhalb von $400 \mathrm{~K}$ setzt die Entmischung ein [15].

Insgesamt haben die in-situ-Untersuchungen gezeigt, daß sich sowohl mit Ratenmessungen als auch mittels AES Oberflächenkonzentrationen in Abhängigkeit von der deponierten Schichtdicke bestimmen lassen. Dabei sind die aus den Ratenmessungen berechneten Konzentrationsverläufe steiler, was auf die nur näherungsweise gültigen Annahmen (Sputteryield unabhängig von der Konzentration) des Modells zurückgeführt wurde. Bei der Deposition von Fe auf Ag ist der Verlauf der Oberflächenkonzentration im Rahmen der Auflösung der AES mit einem scharfen Übergang verträglich (d. h. Breite des Übergangs $<0,7 \mathrm{~nm}$ ). Bei der Deposition von $\mathrm{Cu}$ auf $\mathrm{Ag}, \mathrm{Ag}$ auf $\mathrm{Cu}$ und $\mathrm{Ag}$ auf $\mathrm{Fe}$ zeigen sich nur geringe quantitative Unterschiede im insgesamt flacheren Konzentrationsverlauf. Im Vergleich mit dem vorgestellten Modell zur Implantation der schnellen Ionen ergab sich ein stark asymmetrischer Konzentrationsverlauf über die Grenzfläche, mit einem schnellen Anstieg der Konzentration um $80 \%$ innerhalb von $1 \mathrm{~nm}$ und einem daran anschließenden flacheren Verlauf über mehrere Nanometer. Dies sollte sich in den im folgenden vorgestellten exsitu-Untersuchungen durch eine entsprechend große Breite der Grenzfläche bemerkbar machen. 


\section{Ergebnisse der ex-situ-Untersuchungen}

Im Unterschied $\mathrm{zu}$ den in-situ-Untersuchungen, die stets die Oberfläche der aufwachsenden Schicht beurteilen, dienen die ex-situ-Messungen der Charakterisierung der Proben als Ganzes, insbesondere der inneren Grenzflächen. Sie vervollständigen die Informationen über die Filmeigenschaften, denn aufgrund von unter der Oberfläche stattfindenden Prozessen während des Wachstums der Schicht und eventuellen Veränderungen nach ihrer Herstellung kann man die Schichteigenschaften nicht einfach als Summe der in-situ-Beobachtungen der Oberfläche auffassen.

Die ex-situ-Untersuchungen konzentrierten sich auf die folgenden Methoden: Die Röntgendiffraktometrie im Kleinwinkelbereich dient der Bestimmung von Schichtdicken und Grenzflächenrauhigkeiten bzw. -durchmischung, während die Röntgendiffraktometrie im Weitwinkelbereich Information zu Kristallstruktur und Epitaxiebeziehungen zwischen Film und Substrat liefert. Außerdem wurde Mößbauerspektroskopie zur Untersuchung von Fe-Ag-Schichtpaketen angewendet, um die Verteilung der Ag-Atome im Fe zu bestimmen.

\subsection{Röntgendiffraktometrie}

Für die Kleinwinkel-Röntgendiffraktometrie wurden zunächst Einzelschichten aus $\mathrm{Cu}$, $\mathrm{Ag}$ und $\mathrm{Fe}$ auf $\mathrm{Si}(111)$ und $\mathrm{Si}(100)$-Substraten präpariert. Einzelschichten besitzen nur zwei Grenzflächen, eine zwischen Substrat und Schicht sowie die Oberfläche, und bilden so das einfachste zu untersuchende Schichtsystem. Durch die Untersuchung von Schichten unterschiedlicher Dicke läßt sich die Entwicklung der Oberflächenrauhigkeit mit der Schichtdicke bestimmen. Nach (2-14) ist es außerdem möglich, aus dem kritischen Winkel für Totalreflexion die Elektronendichte zu bestimmen, die für ein gegebenes Element proportional zur Dichte der Schicht ist.

Aufbauend auf den Einzelschichten wurden Doppelschichten aus $\mathrm{Cu}-\mathrm{Ag}$ deponiert, wodurch die $\mathrm{Cu}$ - bzw. Ag-Oberfläche der Einzelschicht jetzt zur intermetallischen Grenzfläche (Cu-Ag bzw. Ag-Cu) wird. Ein Vergleich der Rauhigkeit der Einzelschicht mit der Grenzflächenbreite in der Doppelschicht kann so Informationen über die Veränderung der Rauhigkeit und die Stärke einer zusätzlichen Durchmischung durch die bei der Laserdeposition auftretenden schnellen Ionen liefern. 
Für den komplizierteren Fall der $\mathrm{Cu}$-Ag-Schichtpakete mit sehr vielen Grenzflächen läßt sich nur ein Mittelwert der Rauhigkeiten über gleichwertige Grenzflächen $(\mathrm{Cu}-\mathrm{Ag}$ und $\mathrm{Ag}-\mathrm{Cu}$ sind unterscheidbar) angeben.

\subsubsection{Einzelschichten}

In Abbildung 4-1 bis 4-3 sind die Ergebnisse der spekularen Streuung an $\mathrm{Cu}, \mathrm{Ag}$ und $\mathrm{Fe}$ auf Si dargestellt. Man erkennt zunächst das steile Abfallen der Intensität des Primärstrahls und das darauf folgende Ansteigen bis in das Plateau der Totalreflexion. $\mathrm{Da}$ die Intensität der Totalreflexion nicht von $0^{\circ}$ bis zum kritischen Winkel konstant bleibt, liegt an der endlichen Ausdehnung der Proben. Da der Röntgenstrahl eine gewisse Divergenz hat $\left(1 / 32^{\circ}\right.$ für die hier beschriebenen Messungen), ist bei kleinen Winkeln die beleuchtete Fläche größer als die Probe selbst, was zu einem entsprechenden Intensitätsabfall führt. Bei der Berechnung der Spektren wird eine unendliche Ausdehnung der Probe angenommen ${ }^{6}$, weshalb die simulierten Kurven diesen Verlauf nicht zeigen.

Nachdem der kritische Winkel für Totalreflexion überschritten ist, dringt die Röntgenstrahlung merklich in die Probe ein und die Intensität wird nun von den Materialeigenschaften und der Grenzflächen- bzw. Oberflächenbeschaffenheit bestimmt. Abbildung 4-1a zeigt zunächst das Spektrum eines Si(100)-Substrates, wie es für diese Untersuchungen verwendet wurde. Der Intensitätsverlauf ist sowohl mit einer nicht oxidierten Oberfläche des Si mit 1,2 nm Rauhigkeit als auch mit einer 2,9 nm dicken $\mathrm{SiO}_{2}$-Schicht mit 1,4 nm Rauhigkeit verträglich, wobei die Breite der $\mathrm{Si}_{-} \mathrm{SiO}_{2}$ Grenzfläche 0,5 nm beträgt. Da eine Oxidation von Si an Luft unvermeidlich ist [54], kommt nur die zweite Möglichkeit in Betracht.

Abbildung 4-1b bis j zeigen Spektren für $\mathrm{Cu}$ verschiedener Dicke auf Si(100). Man sieht zunächst, daß sich der Winkel für Totalreflexion deutlich vergrößert, da $\mathrm{Cu}$ mit $10,5 \mathrm{~g} / \mathrm{cm}^{3}$ eine höhere Dichte als Si mit $2,3 \mathrm{~g} / \mathrm{cm}^{3}$ hat.

\footnotetext{
${ }^{6}$ Im typischen industriellen Anwendungsfall von großen Wafern ist diese Bedingung praktisch immer erfüllt.
} 


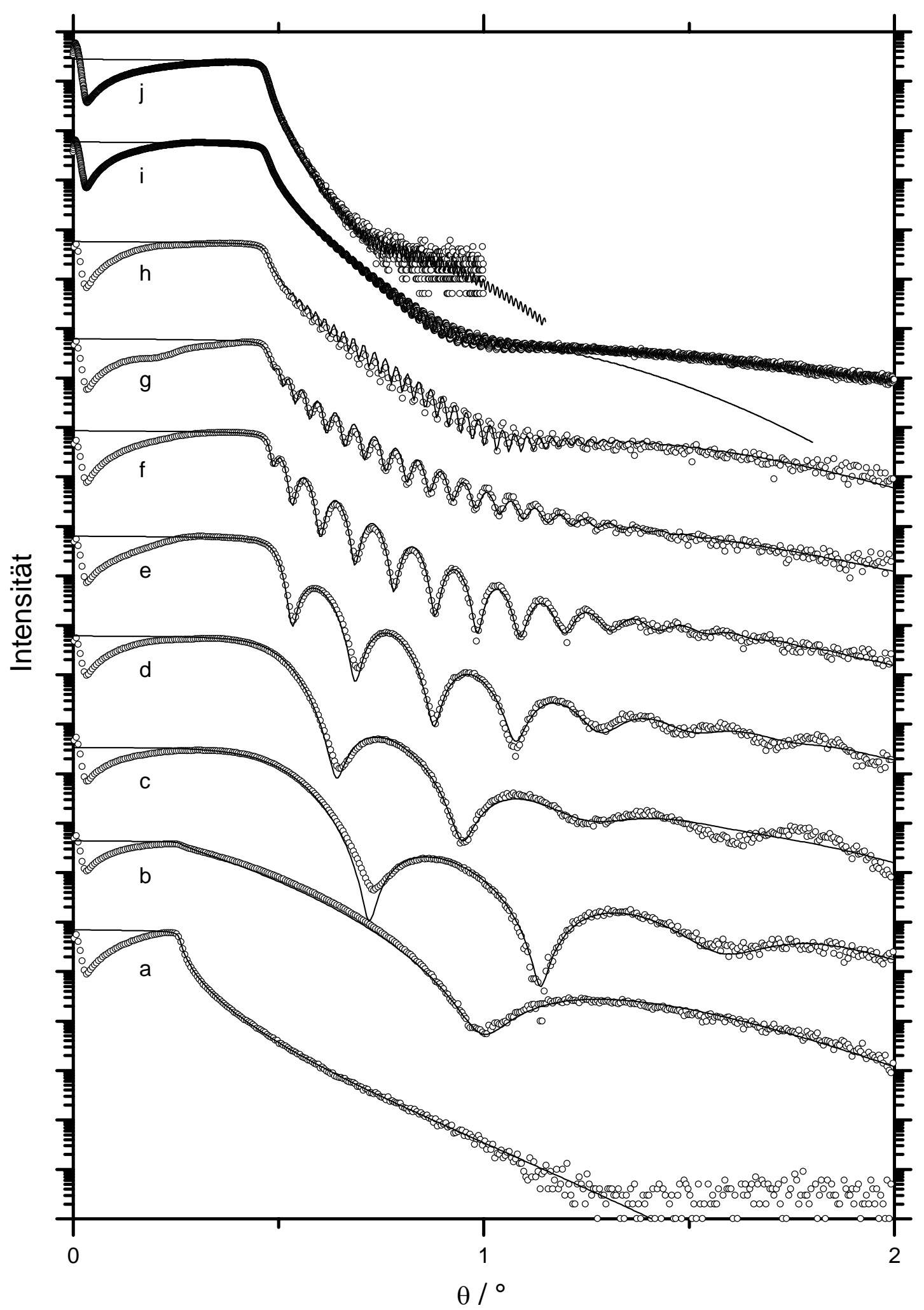

Abbildung 4-1: Kleinwinkel-Röntgendiffraktogramme von $C u$ verschiedener Dicke auf Si(100) in logarithmischer Auftragung; offene Kreise: Meßdaten, durchgezogene Linie: Fit; die einzelnen Kurven sind jeweils um einen Faktor 100 verschoben; a) Si(100)Substrat, Cu-Schichtdicke in b) $1,8 \mathrm{~nm}$, c) 7,5 nm, d) 9,5 nm, e) $18,4 \mathrm{~nm}, f) 39,0 \mathrm{~nm}$, g) $75,1 \mathrm{~nm}, h) 148 \mathrm{~nm}$, i) $231 \mathrm{~nm}$, j) $380 \mathrm{~nm}$ 


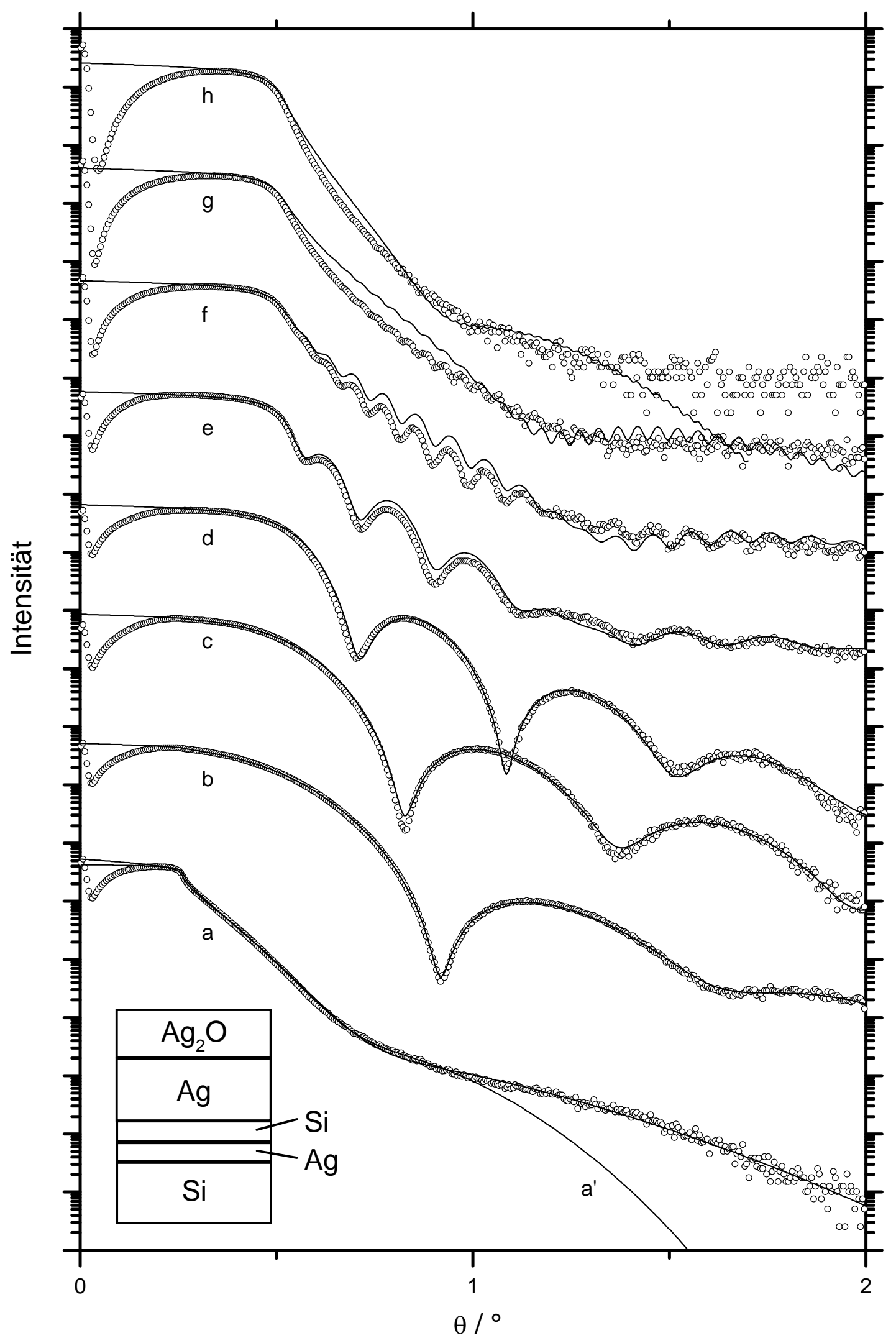

Abbildung 4-2: Kleinwinkel-Röntgendiffraktogramme von Ag-Einzelschichten auf Si(111) (vgl. Abb. 4.1); a) 0,6 nm, b) 3,3 nm, c) 4,5 nm, d) 9,0 nm, e) $18,7 \mathrm{~nm}$, f) 46,4 nm, g) 93,1 nm, h) $184 \mathrm{~nm}$; durchgezogene Linie: Simulation, a') Simulation mit Schichtfolge $\mathrm{Si}-\mathrm{Ag}-\mathrm{Ag}_{2} \mathrm{O}$ 


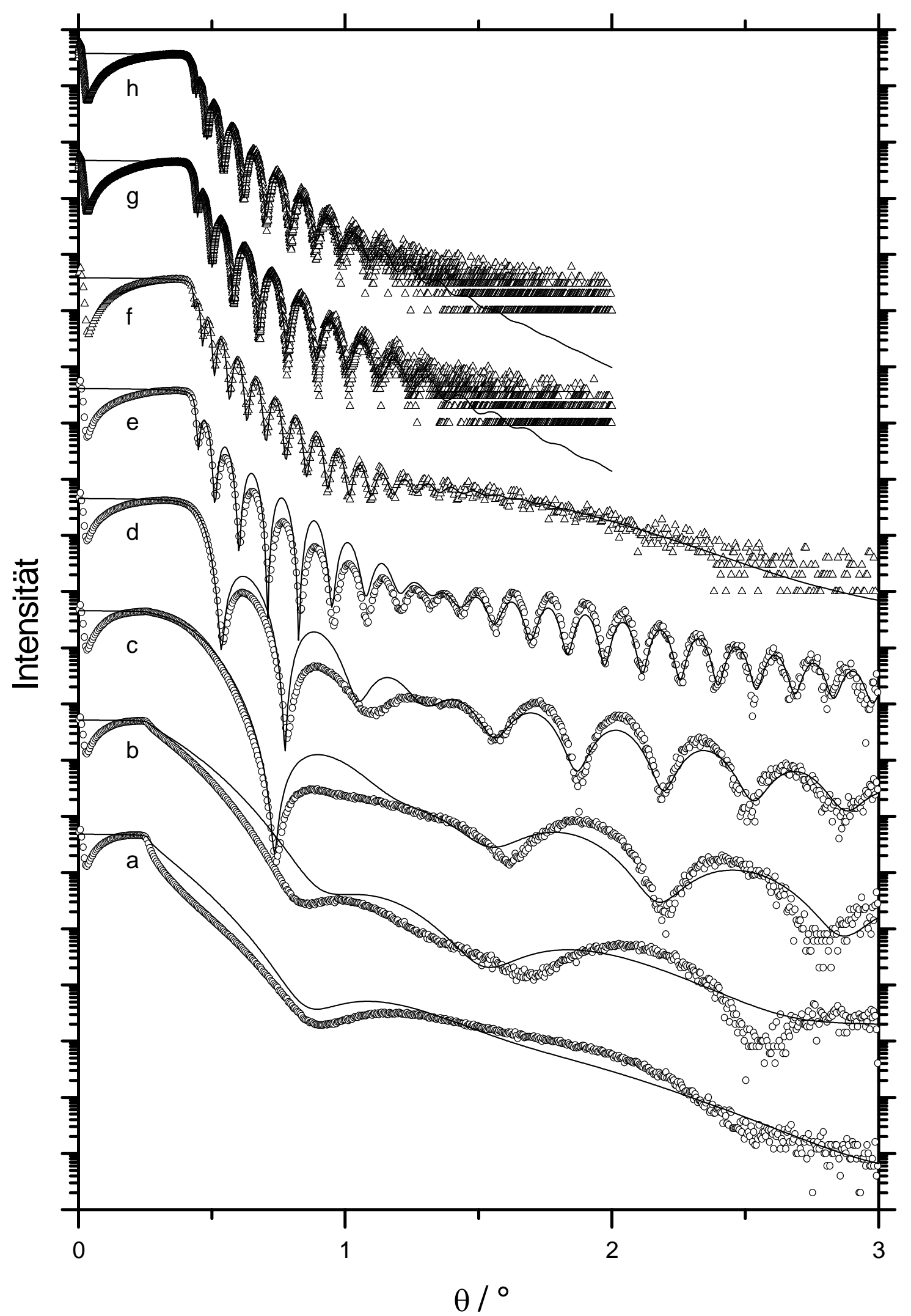

Abbildung 4-3: Kleinwinkel-Röntgendiffraktogramme von Fe-Einzelschichten auf Si(111) - a) bis e) und auf Si(100) - f) bis h); a) 1,7 nm, b) 3,8 nm, c) 7,2 $\mathrm{nm}$, d) $14,4 \mathrm{~nm}$, e) $34,8 \mathrm{~nm}, f) 53,0 \mathrm{~nm}, \mathrm{~g}) 37,0 \mathrm{~nm}, \mathrm{~h}) 44,2 \mathrm{~nm}$ 
Als auffälligstes Merkmal sind allerdings die Schichtdickenoszillationen zu erkennen, die mit größer werdender Schichtdicke enger zusammen liegen, bis sie ab etwa $300 \mathrm{~nm}$ Schichtdicke nicht mehr aufzulösen sind. Die Position der Maxima dieser Oszillationen ist über eine modifizierte Bragg-Beziehung an die Schichtdicke geknüpft:

$$
n \lambda=2 d \sqrt{\sin ^{2} \theta-\sin ^{2} \theta}
$$

Dabei ist $\lambda=0,179 \mathrm{~nm}$ die Wellenlänge der verwendeten Co- $\mathrm{K}_{\alpha}$-Strahlung, $d$ die Schichtdicke, $\theta$ der Winkel des Maximums der Ordnung $n$ und $\theta_{c}$ der kritische Winkel für Totalreflexion, dem das Maximum nullter Ordnung entspricht.

Nach Umstellung von (4-1) zu

$$
\sin ^{2} \theta=\frac{\lambda^{2}}{4 d^{2}} n^{2}+\sin ^{2} \theta_{c}
$$

wird ersichtlich, daß eine Auftragung von $\sin ^{2} \theta$ gegen $n^{2}$ eine Gerade ergibt, aus deren Steigung $m$ die Schichtdicke bestimmt werden kann:

$$
d=\frac{\lambda}{2 \sqrt{m}}
$$

Der Achsenabschnitt $\sin ^{2} \theta_{c}$ ist an den kritischen Winkel $\theta_{c}$ für Totalreflexion geknüpft, der für $\mathrm{Cu} 0,54^{\circ}$, für $\mathrm{Ag} 0,47^{\circ}$ und für $\mathrm{Fe} 0,48^{\circ}$ beträgt.

Der genaue Verlauf der gemessenen Diffraktogramme, die Amplitude der Schichtdickenoszillationen und ihre Dämpfung mit steigendem Winkel, erlauben durch den Vergleich mit der numerischen Simulation der Streuung die Bestimmung der Grenzflächenrauhigkeit zwischen Substrat und Schicht, der Grenzflächenrauhigkeit zwischen Schicht und Oxidschicht, der Dicke der Oxidschicht und der Oberflächenrauhigkeit der Oxidschicht. Der Begriff „Rauhigkeit“" beinhaltet in diesem Fall sowohl morphologische Rauhigkeit als auch chemische Rauhigkeit (d.h. Durchmischung), da die spekulare Streuung von beiden Formen gleich beeinflußt wird. Die numerischen Werte für Rauhigkeiten sind somit als Summe aus morphologischer Rauhigkeit und Durchmischung zu verstehen. Eine getrennte Messung der beiden Größen wird erst durch die diffuse Streuung möglich, deren Interpretation deutlich komplexer ist.

Die simulierten Diffraktogramme für die verschiedenen Cu-Schichtdicken auf $\mathrm{Si}(100)$ sind zusammen mit den gemessenen Kurven in Abbildung 4-1b bis $\mathrm{j}$ aufgetragen. Im 


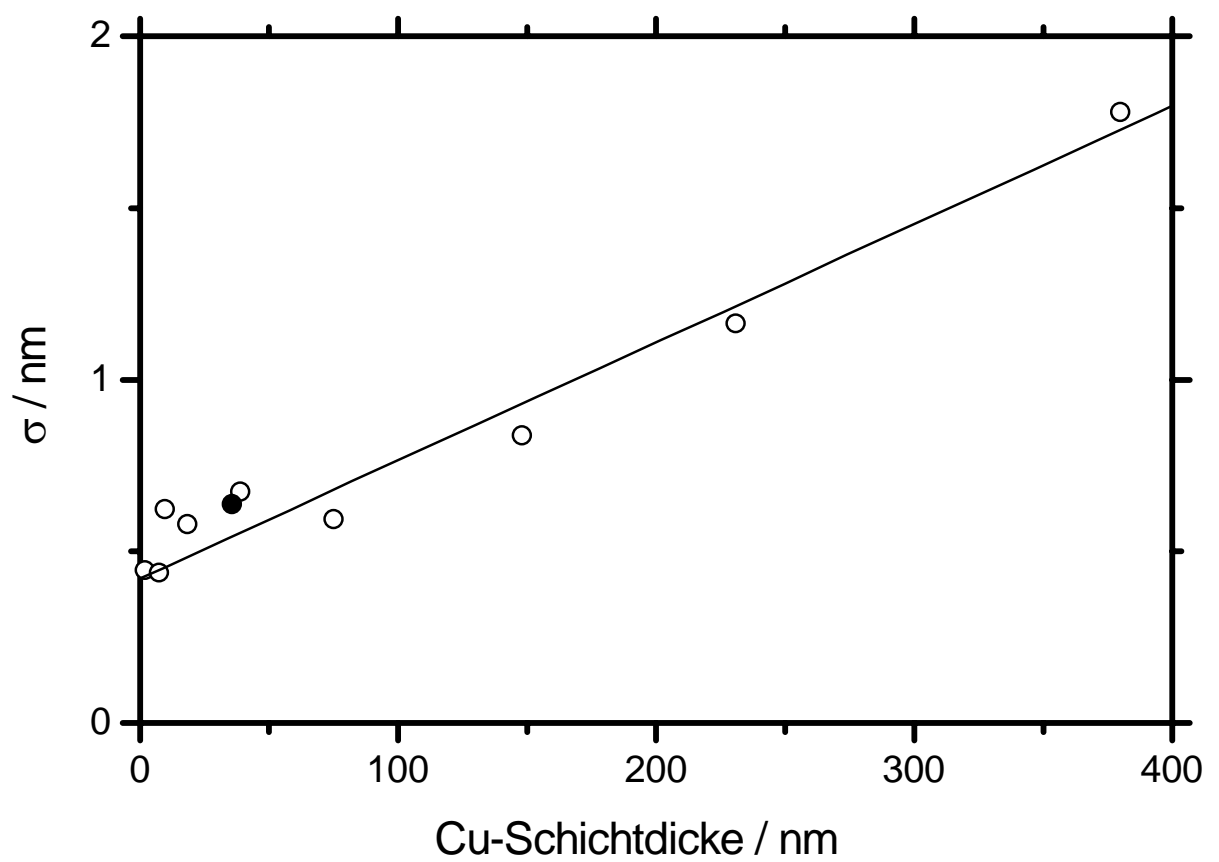

Abbildung 4-4: Aus der Simulation gewonnene Oberflächenrauhigkeit der CuSchichten auf Si(100) (offene Symbole) und Si(111) (geschlossenes Symbol). Die durchgezogene Linie ist ein linearer Fit der Meßpunkte.

Bereich zwischen kritischem Winkel und etwa $1,2^{\circ}$ ist eine gute Übereinstimmung mit den Messungen zu beobachten, während für größere Winkel bei der $231 \mathrm{~nm}$ dicken $\mathrm{Cu}$ Schicht die gemessene Intensität deutlich höher ist als die berechnete und bei den 9,5 und 18,4 nm dicken Schichten die Oszillationsamplitude und -periode nicht mehr übereinstimmt. Als Ursache hierfür kommt eine inhomogene Schichtdickenverteilung in Frage, da während der Messung der Röntgenstrahl mit größer werdendem Winkel eine kleiner werdende Fläche in der Mitte der Probe bestrahlt und so nicht mehr über die gesamte Schicht gemittelt wird. Bei einer Deposition auf ein rotierendes Substrat wäre z. B. eine radial nach außen abnehmenden Schichtdicke denkbar, was zu einer kleineren Oszillationsperiode bei größeren Winkeln führen würde.

Aus den Simulationsparametern (Schichtdicken bzw. Rauhigkeiten) der in Abbildung 4-1 dargestellten Kurven lassen sich folgende Aussagen gewinnen: Alle Proben zeigen eine annähernd konstante Si-Cu-Grenzflächenrauhigkeit von 1,2 bis 1,7 nm, eine Dicke von 2,4 bis 3,4 nm für die Oxidschicht und mit der Schichtdicke ansteigende und praktisch gleich große Rauhigkeiten für die $\mathrm{Cu}-\mathrm{CuO}-$ Grenzfläche und die $\mathrm{CuO}$ Oberfläche. Dieser Anstieg der Oberflächenrauhigkeit mit der Schichtdicke (Abbildung 


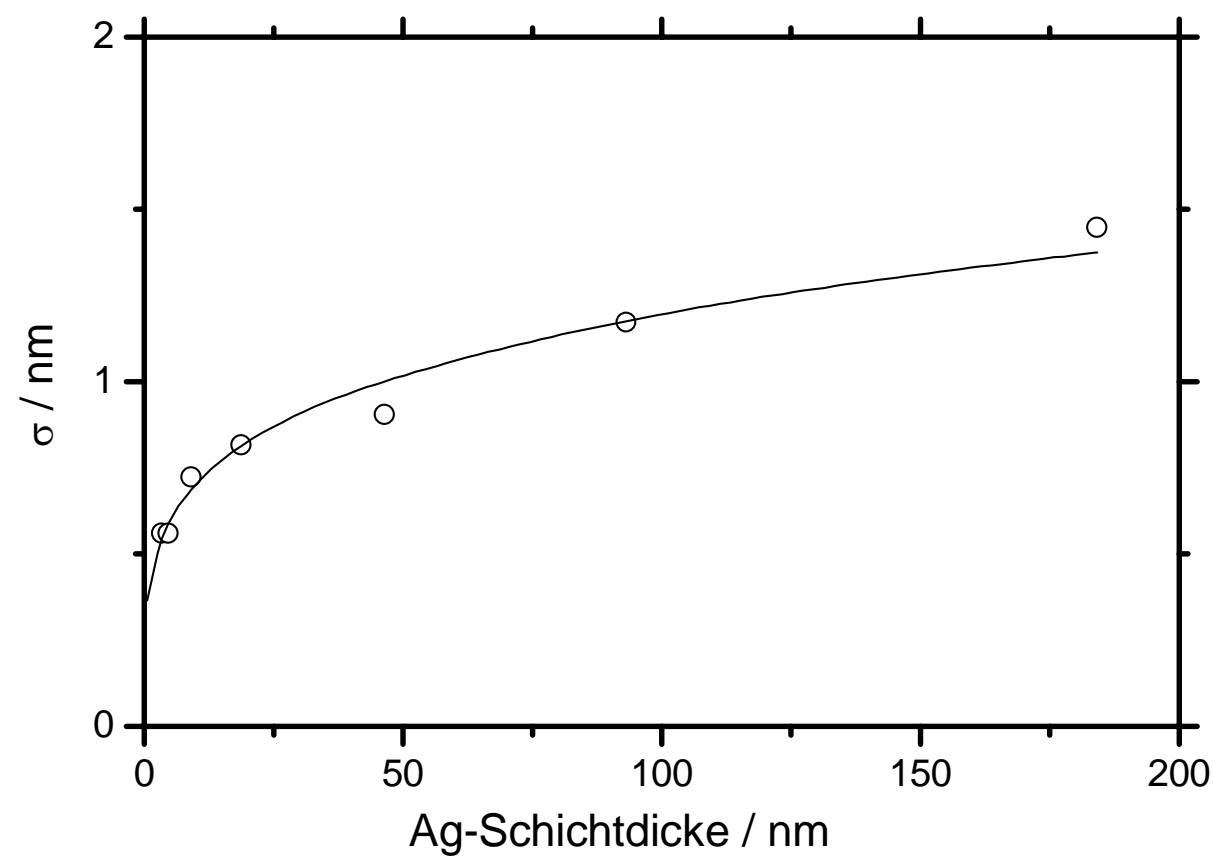

Abbildung 4-5: Aus der Simulation gewonnene Oberflächenrauhigkeit der AgSchichten auf Si(100), durchgezogene Linie: Fit (siehe Text)

4-4) zeigt einen linearen Verlauf im untersuchten Bereich. Eine Sättigung ist nicht zu erkennen. Ein linearer Fit der Oberflächenrauhigkeit liefert folgenden Zusammenhang:

$$
\sigma_{C u}=0,39 \mathrm{~nm}+0,0054 \cdot d_{C u}
$$

Zusätzlich zu den $\mathrm{Cu}$-Schichten auf $\mathrm{Si}(100)$ sind die Ergebnisse für eine auf $\mathrm{Si}(111)$ deponierte $\mathrm{Cu}$-Schicht in Abbildung 4-4 dargestellt. Es ist keine signifikante Abweichung zu den Schichten auf Si(100) zu erkennen.

Für Ag wird ein qualitativ ähnliches Verhalten beobachtet: gleichbleibende Rauhigkeiten für die Si-Ag-Grenzfläche $(1,6 \mathrm{~nm})$ und die Dicke der Oxidschicht $(2,5 \mathrm{~nm}$ bis $3,3 \mathrm{~nm})$. Wie in Abbildung 4-5 $\mathrm{zu}$ erkennen ist, steigt die Oberflächenrauhigkeit $\sigma_{A g}$ jedoch nicht linear mit der Schichtdicke, sondern folgt in etwa einem $\sqrt[4]{d_{A g}}$ - Gesetz:

$$
\sigma_{A g}=0,41 \mathrm{~nm} \cdot\left(d_{A g} / \mathrm{nm}\right)^{0,23}
$$

Im Fall der Fe-Schichten ergeben sich aufgrund der weniger guten Übereinstimmung der berechneten Diffraktogramme mit den gemessenen (Abbildung 4-3) stärker schwankende Grenzflächenbreiten. Die Si-Fe-Grenzfläche ist mit bis zu 1,5 nm Dicke 
vergleichbar mit den $\mathrm{Cu}$ - und Ag-Schichten. Dagegen schwankt die Oberflächenrauhigkeit unabhängig von der Schichtdicke zwischen 0,8 nm und 2,0 nm. Die Oxidschicht ist interessanterweise bei den dünnen Fe-Schichten (unter $5 \mathrm{~nm}$ ) mit bis zu $4 \mathrm{~nm}$ am dicksten und stabilisiert sich bei dickeren Schichten zwischen 2,0 nm und 2,5 nm. Möglicherweise trägt bei den dünnen Schichten der Sauerstoff der $\mathrm{SiO}_{2}$ Schicht zur Oxidation des Fe bei.

\subsubsection{Dichte der Schichten}

Wie in Kap. 2.5 erläutert, wächst der kritische Winkel für Totalreflexion mit der Dichte des Materials. Eine durch Variation der Dichte genaue Anpassung der simulierten Diffraktogramme an die Messung kurz hinter dem Bereich der Totalreflexion sollte demnach eventuelle Abweichungen von der Bulk-Dichte offenbaren. Es ist bekannt, daß dünne Schichten je nach Herstellungsbedingungen unterschiedlich viel freies Volumen enthalten können [55], so daß ihre Dichte kleiner sein kann als beim massiven Material. Abbildung 4-6 und Abbildung 4-7 zeigen für Ag und $\mathrm{Cu}$, daß hier der umgekehrte Fall zutrifft: Die Dichte ist für kleine Schichtdicken erhöht und relaxiert zum Bulk-Wert für größere Schichtdicken. Bei Ag erfolgt diese Relaxation über einen Bereich von wenigen Nanometern, was auf einen direkten Einfluß der Si-Ag-Grenzfläche schließen läßt. Eine Abschätzung aus der Ag-Dichte an der Grenzfläche liefert einen um 3,3\% reduzierten Atomabstand in der Ebene, wenn man den Gitterabstand senkrecht zur Ebene als konstant annimmt. Im Fall der $\mathrm{Cu}$-Schichten erreicht die Dichte erst bei hohen Schichtdicken von über $200 \mathrm{~nm}$ ihren Normalwert. Damit müssen die Cu-Schichten im gesamten Volumen komprimiert sein, ohne daß die Si-Cu-Grenzfläche diese Gitterkompression stabilisiert. Allerdings ist die Erhöhung der Dichte viel geringer als im Fall der Ag-Schichten. Eine ähnliche Abschätzung wie oben für die in-planeGitterkonstante liefert nur eine Reduzierung von $0,16 \%$ bei $\mathrm{Cu}$. Diese geringe Kompression paßt ins Bild des sog. shot peenings, das eine kontinuierliche Verdichtung durch implantierte energetische Ionen beim Wachstum laserdeponierter Schichten beschreibt [16].

Ebenfalls simuliert wurde die Dichte der Fe-Schichten, die aber von Probe zu Probe stark schwankt und keine Tendenz erkennen läßt. Bei allen derartigen Berechnungen der Dichte muß darauf hingewiesen werden, daß die erhaltenen Werte mit hohen Fehlern behaftet und nur als Richtwerte zu verstehen sind. 


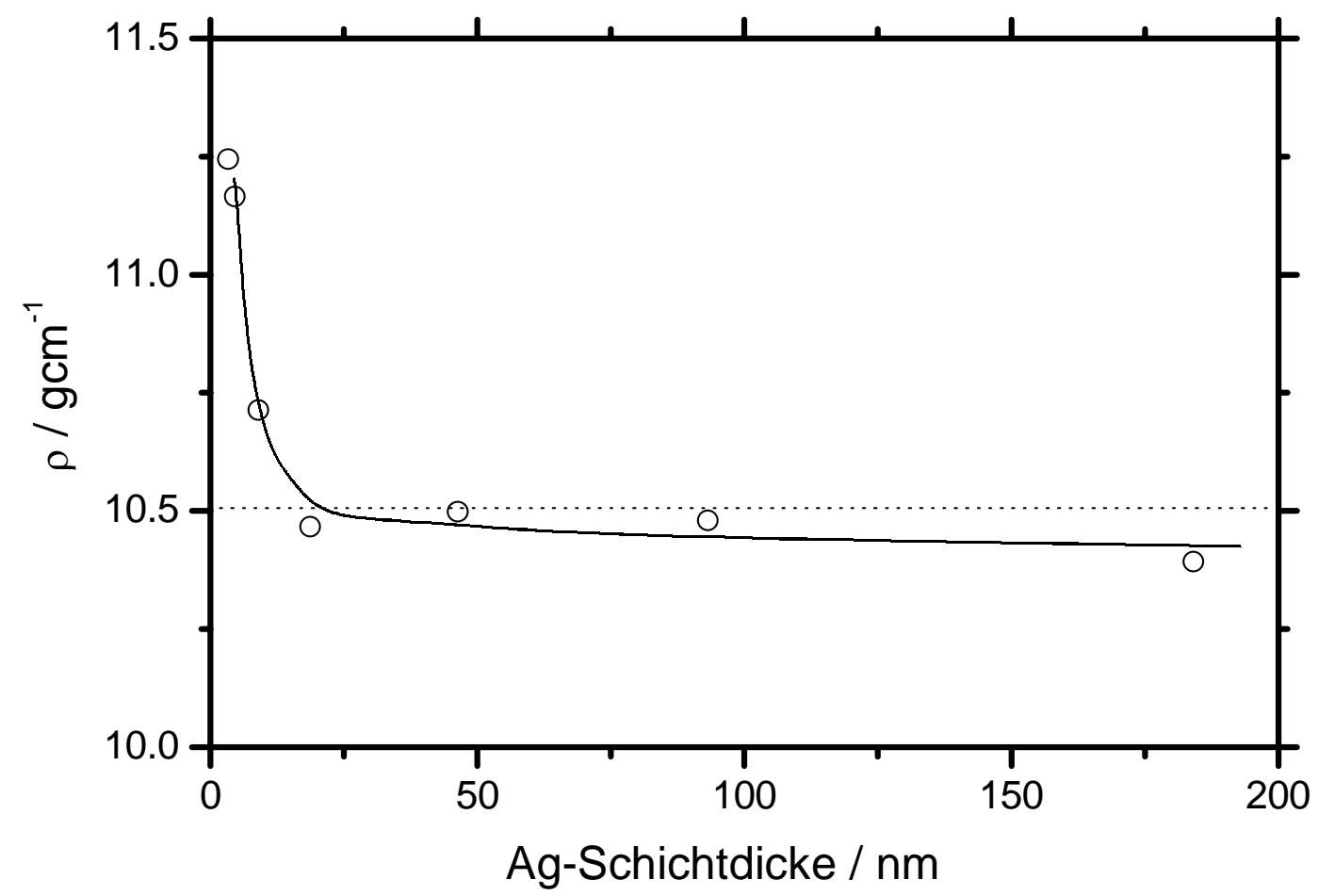

Abbildung 4-6: Dichte der Ag-Schichten; Die eingezeichnete Kurve dient nur der qualitativen Darstellung des Verlaufs.

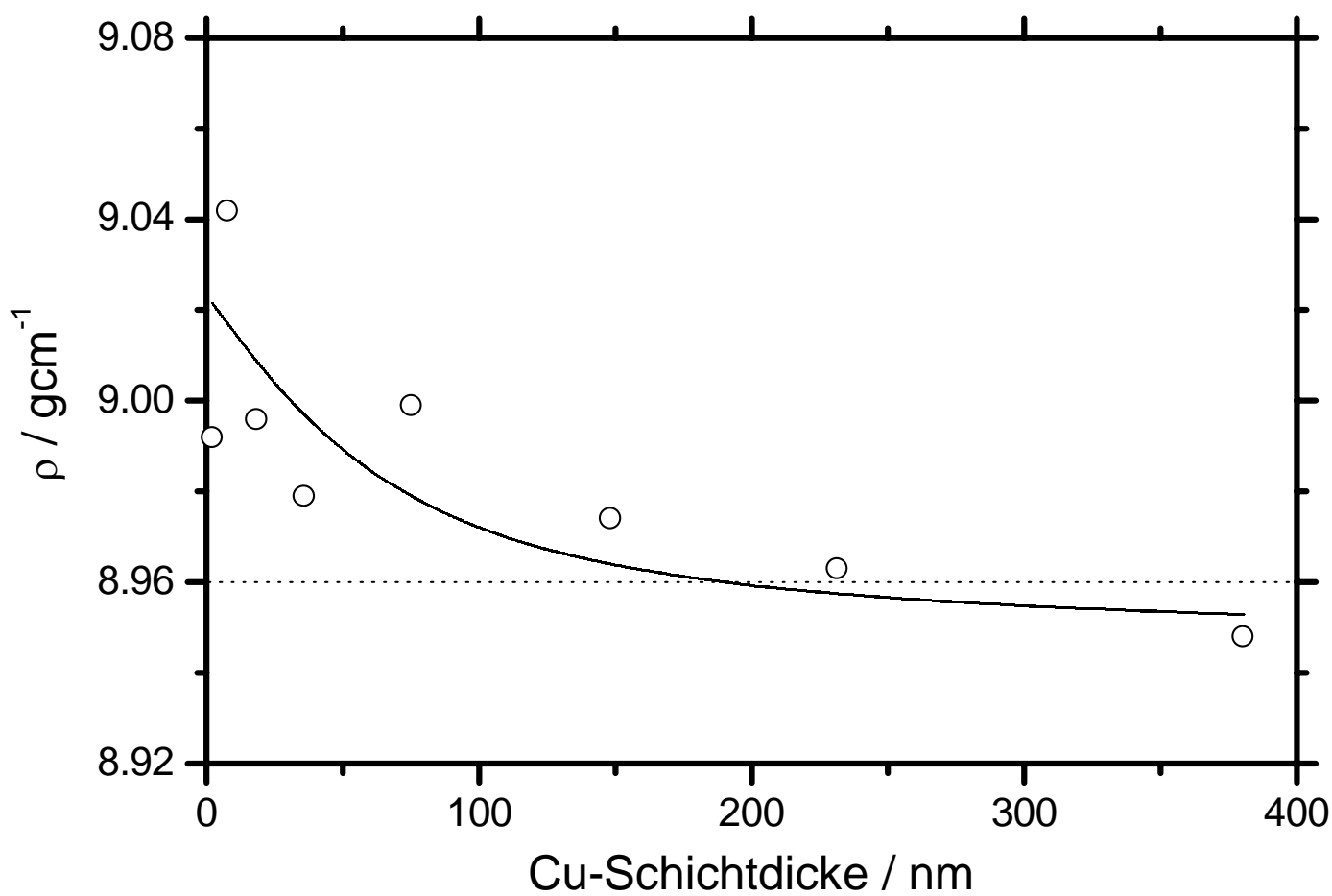

Abbildung 4-7: Dichte der Cu-Schichten; Die eingezeichnete Kurve dient nur der qualitativen Darstellung des Verlaufs. 


\subsubsection{Mehrfachschichten}

Um die Grenzflächen zwischen verschiedenen Metallen untersuchen zu können, wurden Doppelschichten aus $\mathrm{Cu}$ und $\mathrm{Ag}$ präpariert. Dabei wurden zunächst auf $\mathrm{Si}(100)$ Substrate $50 \mathrm{~nm}$ dicke Schichten aus $\mathrm{Cu}$ (bzw. Ag) aufgebracht, die jeweils mit einer Schicht aus Ag (bzw. Cu) verschiedener Dicke überdeckt wurden. Die Ergebnisse der Kleinwinkel-Röntgenstreuung sowie die berechneten Diffraktogramme sind in Abbildung 4-8 dargestellt. Je nach Schichtfolge sind die Ergebnisse sehr unterschiedlich. Wird auf das $\mathrm{Si}(100)$-Substrat zunächst $\mathrm{Cu}$ und dann Ag deponiert, ergeben sich die Kurven d) bis f). Die Intensität fällt in diesen Fällen monoton ab, wobei die überlagerten Schichtdickenoszillationen bis zu hohen Winkeln zu beobachten sind, was auf eine geringe Substratrauhigkeit bzw. Durchmischung der Si-CuGrenzfläche schließen läßt. Im Gegensatz dazu ist die Amplitude der Schichtdickenoszillationen bei der Schichtfolge Si-Ag-Cu, Kurven a) bis c), stärker gedämpft und folglich die Si-Ag-Grenzfläche stärker durchmischt bzw. rauher.

Als weiteres markantes Merkmal der Si-Ag-Cu-Schichten fällt der Anstieg der reflektierten Röntgenintensität bei Winkeln $\theta>1,3^{\circ}$ auf. Für diese zusätzliche langwellige Oszillation muß eine dünne Schicht in der Nähe der Si-Ag-Grenzfläche verantwortlich sein. Die Simulation zeigt konsequenterweise nur dann die in Abbildung 4-8 dargestellte sehr gute Übereinstimmung mit den Messungen, wenn die Schichtfolge $\mathrm{Si}-\mathrm{Ag}-\mathrm{Si}-\mathrm{Ag}-\mathrm{Cu}-\mathrm{CuO}$ zugrunde gelegt wird. Wird für die Simulation die Schichtfolge $\mathrm{Si}-\mathrm{Ag}-\mathrm{Cu}-\mathrm{CuO}$ angenommen, ist die Übereinstimmung mit den Messungen bei großen Winkeln nicht mehr gut (4-9a'). Für die Si-Cu-Grenzfläche tritt dieses Problem nicht auf, deswegen wird hier die zu erwartende Schichtfolge $\mathrm{Si}-\mathrm{Cu}-\mathrm{Ag}-\mathrm{Ag}_{2} \mathrm{O}$ verwendet (Kurven d bis f).

Die Ergebnisse der Simulation sind eine Grenzflächenrauhigkeit Si-Cu von $0,3 \mathrm{~nm}$ (deutlich geringer als die bei den $\mathrm{Cu}$-Einzelschichten, aber besser mit den Herstellerangaben von 0,4 nm für Oberflächenrauhigkeit der Si-Wafer vereinbar), für Si-Ag eine mit 0,6 nm doppelt so hohe. Da die Substrate dem selben Wafer entstammen und die Proben identisch präpariert wurden, muß es zwischen $\mathrm{Si}$ und $\mathrm{Ag} \mathrm{zu}$ einer stärkeren Reaktion kommen als zwischen $\mathrm{Si}$ und $\mathrm{Cu}$ oder aber das $\mathrm{Ag}$ während der Deposition stärker in das Si implantiert werden, was jedoch von Berechnungen der Eindringtiefe nicht gestützt wird [11]. 


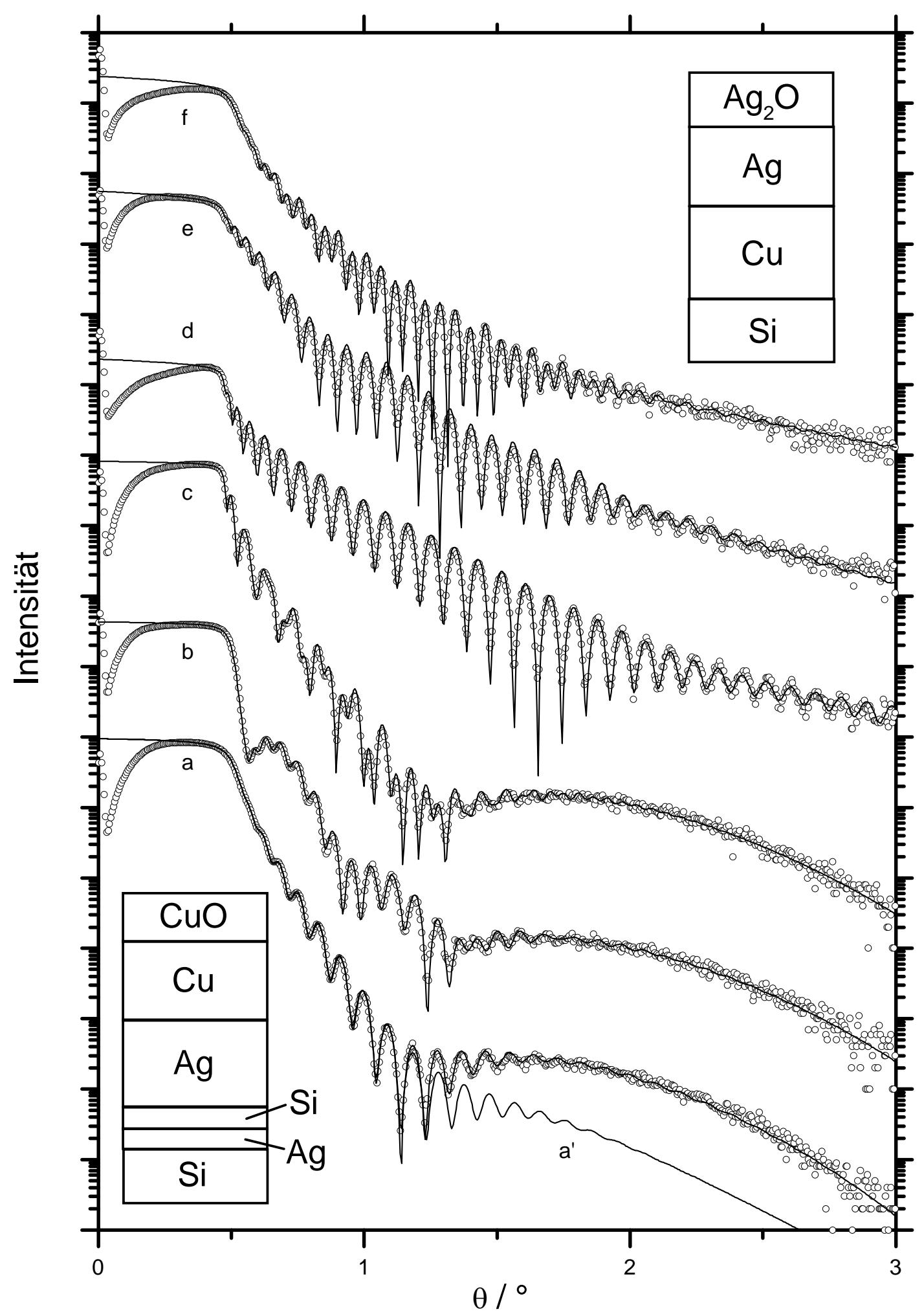

Abbildung 4-8: Kleinwinkel-Röntgenstreuung an Cu-Ag-Doppelschichten auf Si(100) mit der Schichtfolge Si-Ag-Cu (a-c) und Si-Cu-Ag (d-e); a) 50nm Ag-2nm Cu, b)

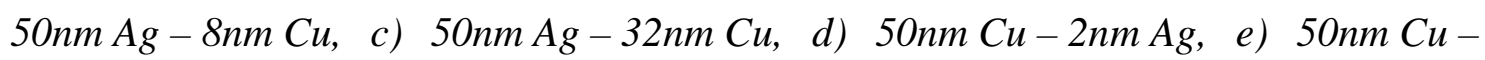
$8 \mathrm{~nm} \mathrm{Ag}$, f) 50nm Cu-32nm Ag; a') bester Fit für die Schichtfolge Si-Ag-Cu-CuO 
Die Breite der Cu-Ag- bzw. der Ag-Cu-Grenzfläche ergibt sich zu 0,8 nm bzw. 1,3 nm. Im Vergleich mit den Oberflächenrauhigkeiten der Einzelschichten gleicher Dicke zeigt sich nur eine minimale Veränderung von $0,1 \mathrm{~nm}$ im Fall von $\mathrm{Cu}$, aber eine Erhöhung der Breite der Ag-Cu-Grenzfläche um 0,4 nm. Dieses Ergebnis bestätigt frühere in-situMessungen der Leitfähigkeit an $\mathrm{Cu}-\mathrm{Ag}$-Schichtpaketen, nach denen bei der Laserdeposition von $\mathrm{Cu}$ und $\mathrm{Ag}$ bei gleicher Energiedichte auf dem Target das $\mathrm{Cu}$ stärker in das Ag implantiert wird als umgekehrt [11]. ${ }^{7}$

\subsubsection{Schichtpakete}

Diese Asymmetrie in der Breite der Grenzflächen läßt sich auch in $\mathrm{Cu}-\mathrm{Ag}$ Schichtpaketen beobachten. Abbildung 4-9 zeigt das Ergebnis der Kleinwinkelstreuung an $20 \mathrm{Cu}$-Ag-Doppelschichten auf $\mathrm{Si}(100)$, wobei Kurve a) der Schichtfolge Si-Ag$\mathrm{Cu} . .$. und Kurve b) der Schichtfolge Si-Cu-Ag... entspricht, mit ansonsten gleichen Parametern.

Die Diffraktogramme verlaufen in beiden Fällen sehr ähnlich, nur die Schichtdickenoszillationen für Winkel oberhalb des Bragg-Peaks bei $1,3^{\circ}$ sind für die Schichtfolge Si-Ag-Cu... stärker gedämpft, was wiederum auf höhere Durchmischung bzw. stärkere Rauhigkeit der Si-Ag-Grenzfläche im Vergleich zu Si-Cu hindeutet. Die Simulation liefert im letzteren Fall eine Grenzflächenbreite von 0,9 nm, für Si-Ag hingegen 1,5 nm. Im Gegensatz zu den oben beschriebenen Doppelschichten wurde bei den Schichtpaketen die Simulation mit der normalen Schichtfolge durchgeführt, da aufgrund der vielen Einzelschichten der Einfluß einer zusätzlichen Zwischenschicht vergleichbarer Dicke nicht mehr von den übrigen Schichten $\mathrm{zu}$ trennen ist. Die gemessenen Kurven zeigen, daß die Schichtdickenoszillationen nicht so gleichförmig verlaufen wie im berechneten Schichtpaket, was schon durch kleine Unregelmäßigkeiten in der Depositionsrate während der Herstellung der Schichten verursacht sein kann. Denn die daraus resultierende Variation in den Dicken der einzelnen Schichten führt mit zunehmender Schichtanzahl zu einer Verringerung der Kohärenz im Schichtpaket.

Auch die Rauhigkeit der einzelnen Schichten ist sicher nicht konstant, wie in dem der Berechnung zugrunde liegenden Modellschichtpaket, sondern nimmt mit dem Abstand

\footnotetext{
${ }^{7}$ Beim Vergleich muß bedacht werden, daß die hier angegebenen Werte RMS-Rauhigkeiten bzw. -Durchmischungen sind, d. h. nur etwa die halbe Grenzflächenbreite darstellen - vgl. Abbildung 2-6.
} 
vom Substrat zu. Deshalb sind die simulierten Grenzflächenbreiten von $0,8 \mathrm{~nm}$ für Cu$\mathrm{Ag}$ und 1,5 nm für $\mathrm{Ag}-\mathrm{Cu}$ als Mittelwert zu verstehen. Es zeigt sich der schon bei den $\mathrm{Cu}$-Ag-Doppelschichten beschriebene Unterschied in der Breite der Grenzflächen.

Ebenfalls unterschiedlich ist die Dicke der einzelnen $\mathrm{Cu}$ - und Ag-Schichten. Deponiert wurden jeweils gleiche Schichtdicken an $\mathrm{Cu}$ und $\mathrm{Ag}$, die Simulation ergibt jedoch 1,6 nm für Ag und 2,6 nm für Cu. Diese Diskrepanz läßt sich ebenfalls mit den für die Laserdeposition typischen energetischen Ionen erklären. Diese werden einerseits implantiert, führen aber andererseits zu einem teilweisen Wiederabsputtern schon deponierten Materials. Da die Sputterrate bei Deposition von $\mathrm{Cu}$ auf Ag höher ist als im umgekehrten Fall, verringert sich die Ag-Schichtdicke um einen bestimmten Betrag [22].

Neben den Messungen der spekular reflektierten Intensität sind in Abbildung 4-9 auch Offset-Messungen mit $0,1^{\circ}$ Verkippung der Probe in der Streuebene dargestellt. Damit liegt die Oberfläche der Probe außerhalb des Winkelbereiches, in dem spekulare Reflexion auftritt ${ }^{8}$, und die Intensität wird durch diffuse Streuung bestimmt. Das Auftreten des Bragg-Peaks auch bei diffuser Streuung ist ein Hinweis auf korrelierte Rauhigkeiten im Schichtpaket [28], d.h. die Rauhigkeiten der unteren Schichten setzen sich vertikal in den darüberliegenden Schichten fort.

\footnotetext{
${ }^{8}$ Mit Rocking-Scans wurde die Breite des spekularen Peaks zu etwa $0,02^{\circ}$ bestimmt.
} 


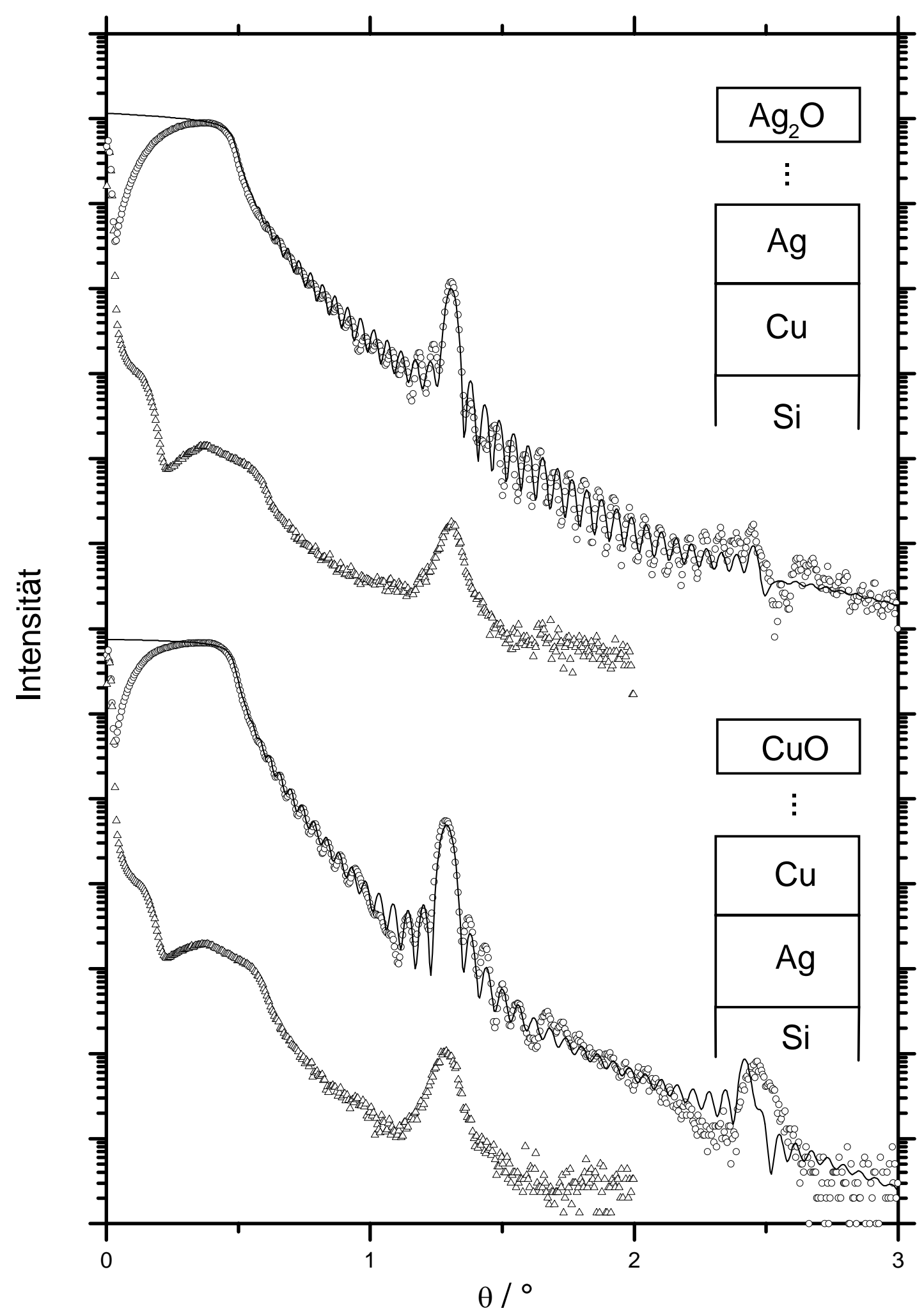

Abbildung 4-9: spekulare Scans (Kreise) und 0,1 ${ }^{\circ}$-Offset-Scans (Dreiecke) der Cu-Ag-

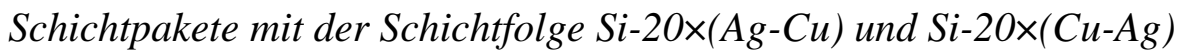




\subsubsection{Kristallographische Orientierung}

An den Cu-Ag-Doppelschichten und -Schichtpaketen wurden Röntgendiffraktogramme im Großwinkelbereich aufgenommen, um Aussagen über die kristallographische Orientierung der Schichten und die Orientierungsbeziehung zum Substrat zu gewinnen. fcc-Metalle wachsen i.a. (111)-orientiert auf amorphen Unterlagen wie der oxidieren SiOberfläche auf, da so die dichtest gepackten Ebenen die Grenzfläche zum Substrat bilden. Abbildung 4-10 zeigt aber, daß in diesem Fall sowohl Ag als auch $\mathrm{Cu}$ auf $\operatorname{Si}(100)$ in der (100)-Orientierung aufwachsen. Zusammen mit der guten in-planeTextur der Schichten (Abbildung 4-11 und 4-12) zeigt dies, daß keine amorphe $\mathrm{SiO}_{2}$ Schicht zwischen der Si(100)-Oberfläche und den deponierten Schichten liegen kann. Auch in einigen anderen Fällen ist eine Epitaxie auf oxidierten Si-Substraten beobachtet

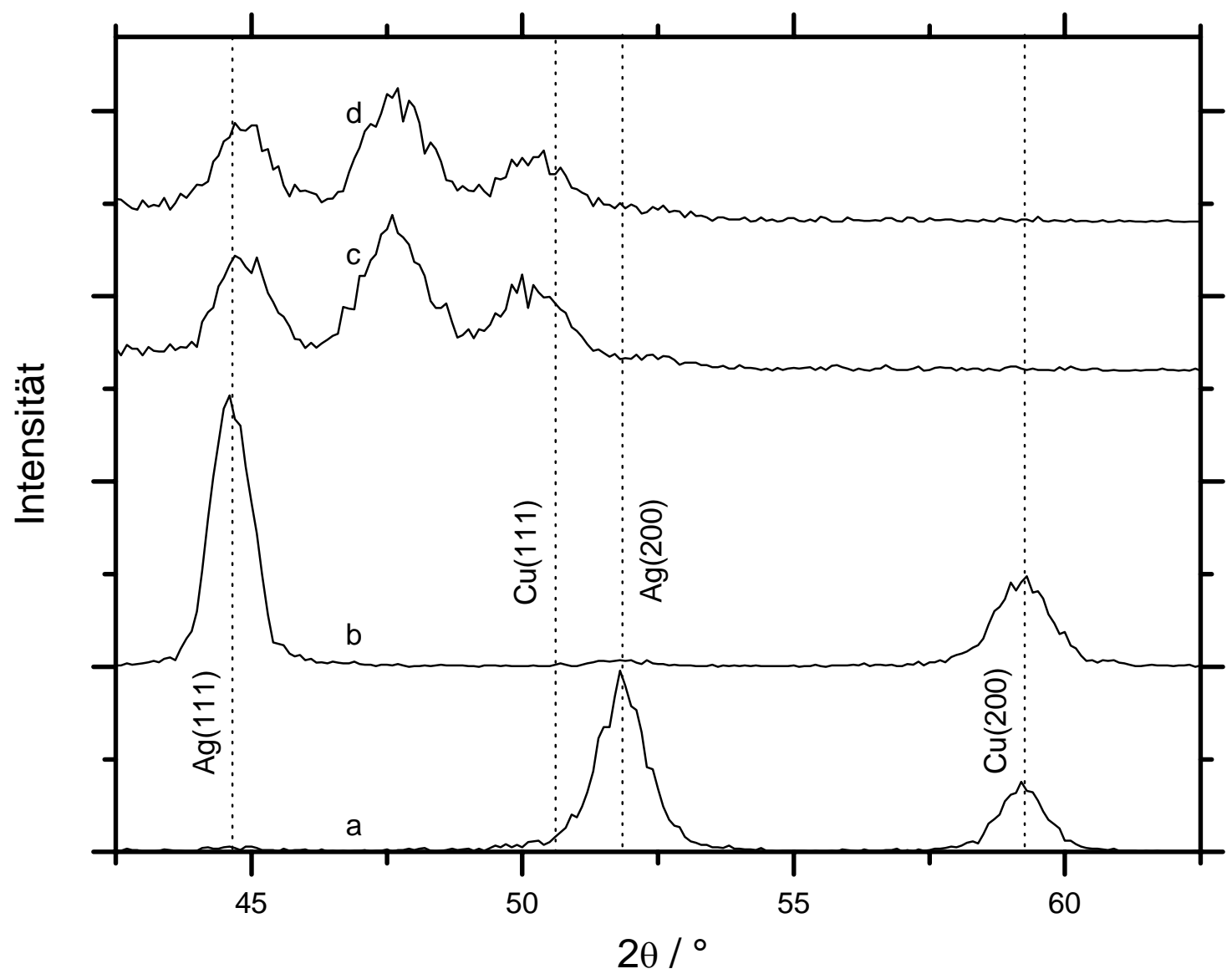

Abbildung 4-10: Großwinkel-Röntgendiffraktogramme der Cu-Ag-Schichten auf Si(100) für die Doppelschichten Si-Ag-Cu (a), Si-Cu-Ag (b) und die Schichtpakete Si-20×(Ag-Cu) (c) und Si-20×(Cu-Ag) (d); gestrichelte Linien markieren die Positionen der Bulk-Reflexe 


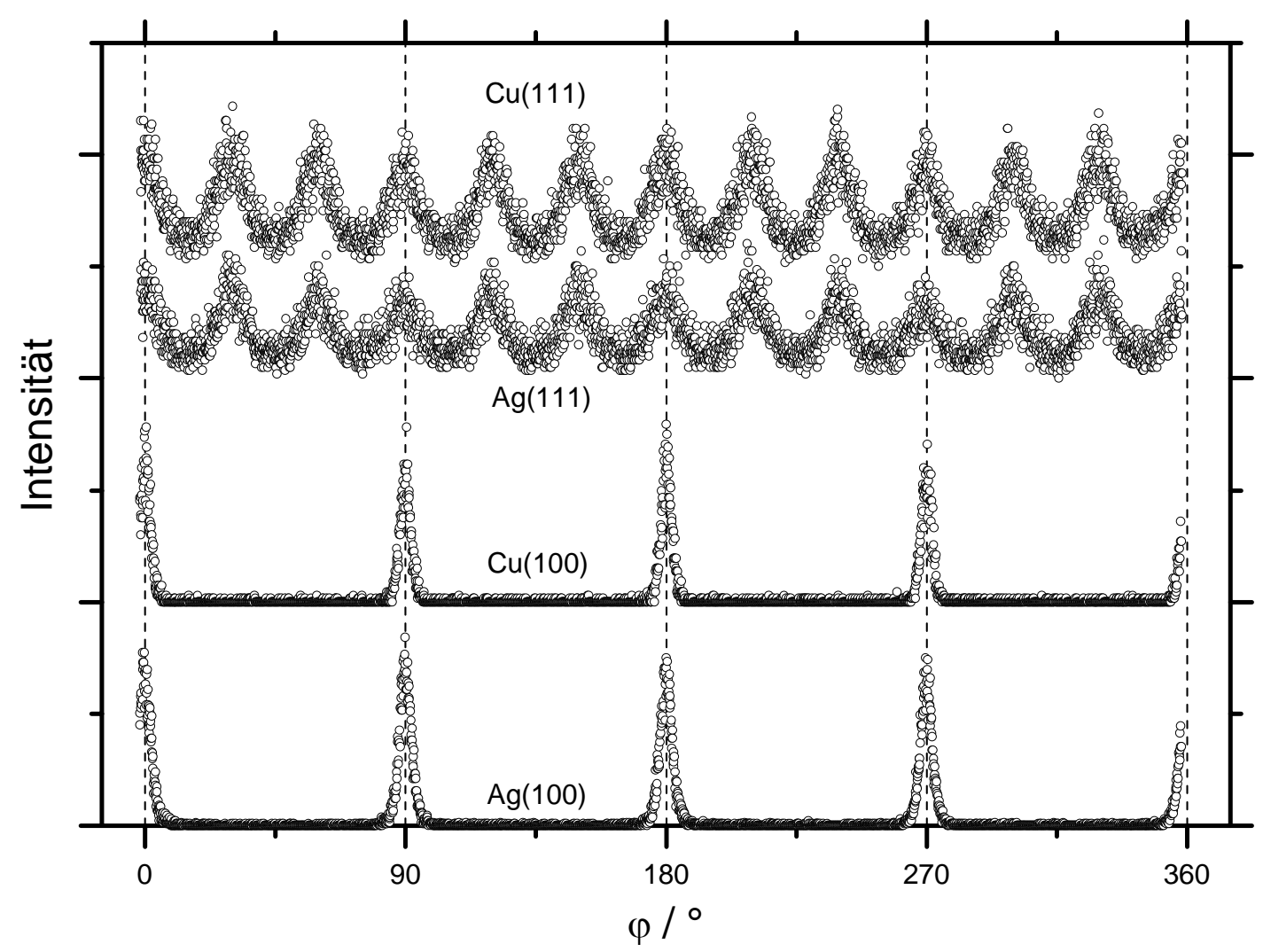

Abbildung 4-11: $\varphi$-Scans des 111-Reflexes für die Doppelschicht Si(100)-Ag-Cu (untere beiden Kurven) und das Schichtpaket Si(100)-20×(Ag-Cu) (obere beiden Kurven); gestrichelte Linien markieren die Position des Substratreflexes

worden [56,57]. Das Verschwinden der $\mathrm{SiO}_{2}$-Schicht läßt sich mit ihrem Absputtern durch die bei der PLD auftretenden Ionen erklären, die mit Energien um $100 \mathrm{eV}$ auf dem Substrat auftreffen [21]. Bei Depositionsmethoden, die ohne energiereiche Teilchen auskommen müssen, ist die vorherige Entfernung der Oxidschicht und eine HTerminierung der Si-Oberfläche durch Ätzen in Flußsäure zur Verhinderung einer schnellen Reoxidation notwendig [58]. In diesen Fällen wird ebenfalls (100)-Wachstum von $\mathrm{Cu}$ [58] und von $\mathrm{Ag}$ [59] auf $\mathrm{Si}(100)$ beobachtet.

Die (100)-Epitaxie überträgt sich im Fall der Schichtfolge Si-Ag-Cu vom Ag auf das $\mathrm{Cu}$, allerdings nicht im umgekehrten Fall. Hier wächst das Ag in seiner ,natürlichen“ (111)-Orientierung auf dem $\mathrm{Cu}$ auf. Damit wird die (100)-Textur beim Übergang von $\mathrm{Cu}$ auf $\mathrm{Ag}$ zerstört, wie sich auch in den $\mathrm{Cu}-\mathrm{Ag}$-Schichtpaketen zeigt (Abbildung 4-11). 


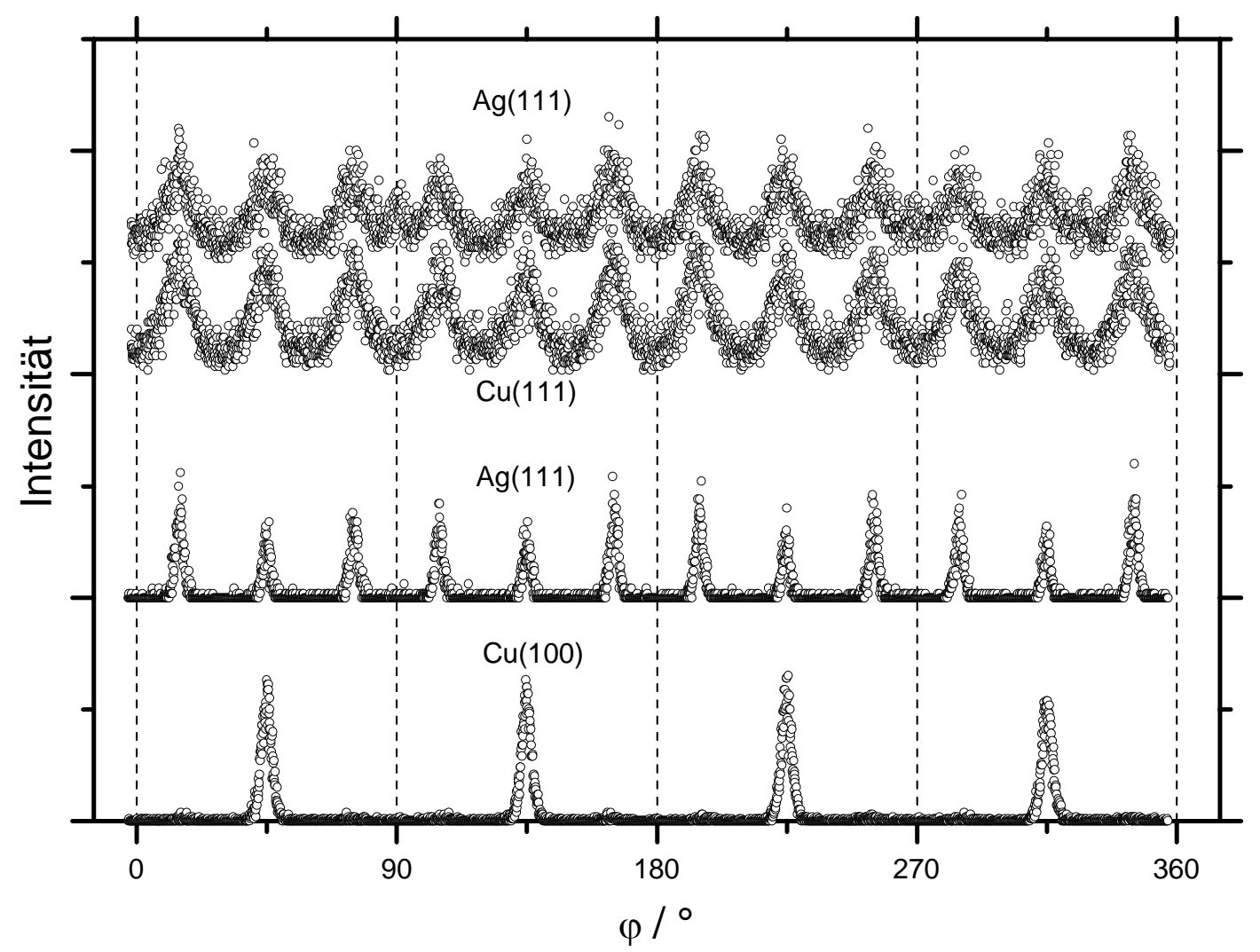

Abbildung 4-12: $\varphi$-Scans des 111-Reflexes für die Doppelschicht Si(100)-Cu-Ag (untere beiden Kurven) und das Schichtpaket Si(100)-20×(Cu-Ag) (obere beiden Kurven); gestrichelte Linien markieren die Position des Substratreflexes

Diese zeigen nur noch die (111)-Orientierung für beide Elemente. ${ }^{9}$ Bei Schichtpaketen wird die gestreute Amplitude durch die Gitterfunktion der periodischen Schichtanordnung moduliert [8], was bei einer Doppelschichtdicke von 4,2 nm zu der beobachteten Satellitenpeakstruktur in Abbildung 4-10 führt.

Zur Bestimmung der Orientierungsbeziehung zwischen Film und Substrat wurden $\varphi-$ Scans des (111)-Reflexes unter verschiedenen Verkippungswinkeln der Probe durchgeführt. Abbildung 4-11 zeigt die Ergebnisse für die Doppelschicht Si-Ag-Cu und das Schichtpaket Si-20×(Ag-Cu). Zunächst wurden die Positionen der (111)-Reflexe des $\operatorname{Si}(100)$-Einkristalls ermittelt, deren Lage durch gestrichelte Linien angedeutet sind. Einer der Reflexe wurde für jede Probe als Referenzposition für die $0^{\circ}$-Richtung festgelegt. Es zeigt sich, daß das Ag mit einer in-plane-Textur von $4,1^{\circ}$ in der selben

\footnotetext{
${ }^{9}$ Die untersten (100)-orientierten Schichten sind mit jeweils $2 \mathrm{~nm}$ zu dünn, um eine merkliche Intensität im (200)-Peak zu hinterlassen.
} 


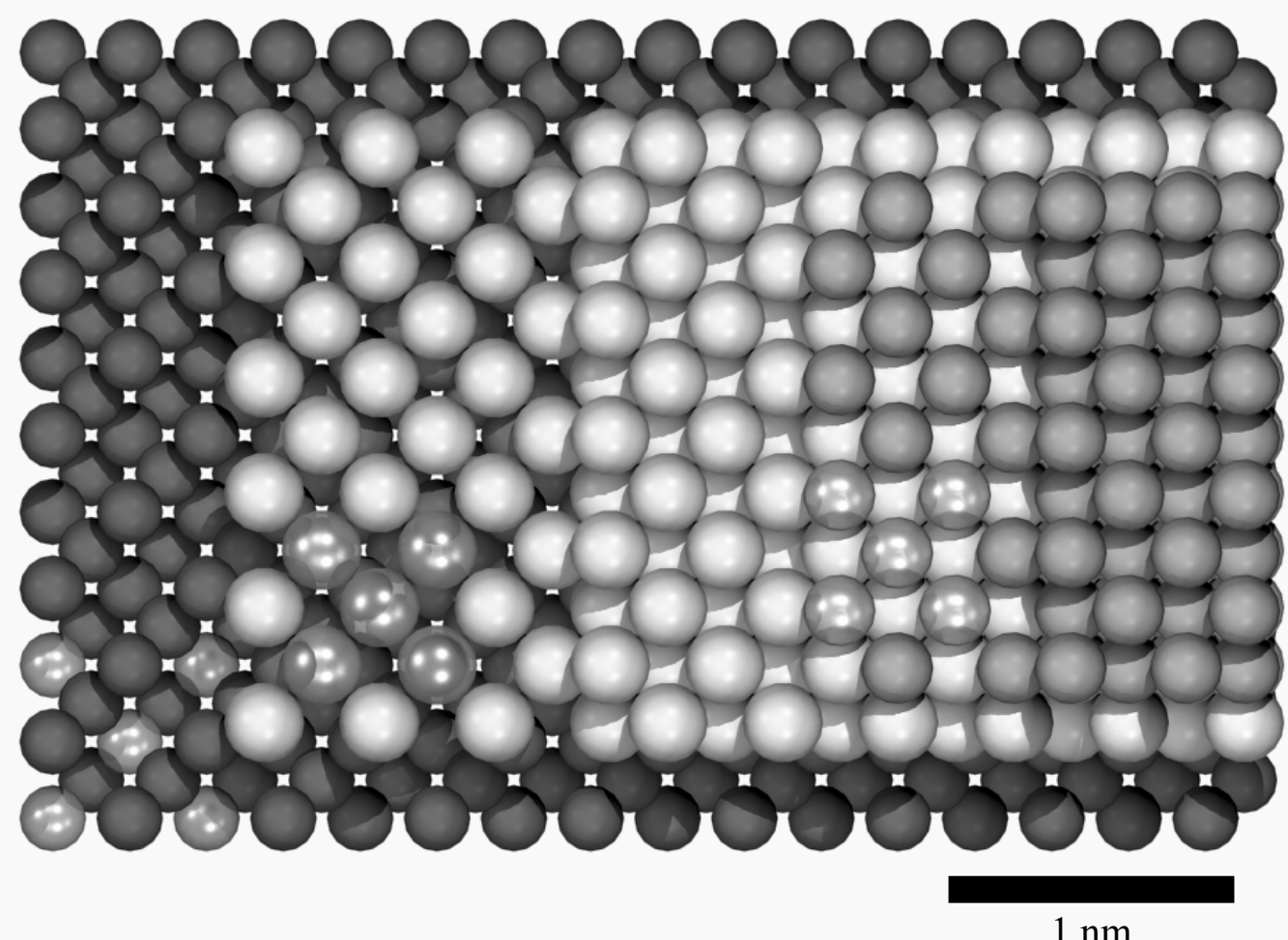

Abbildung 4-13: Si-Ag-Cu-Schichtfolge im Modell harter Kugeln; dunkelgrau Si, hellgrau $\mathrm{Cu}$, weiß Ag; die Grundflächen der Einheitszellen sind jeweils mittels durchsichtiger Kugeln veranschaulicht

Richtung wie das Si weiterwächst, also $[100]_{\mathrm{Ag}} \|[100]_{\mathrm{Si}}$ in der Ebene. Der Gitterparameter von Ag ist mit 0,409 nm um 33\% kleiner als der von Si (0,543 nm), so daß ein direktes Atom-auf-Atom-Wachstum nicht möglich ist. Statt dessen bildet sich ein 4:3-Koinzidenzgitter [60] aus, bei dem nach jeweils 3 Si-Atomen bzw. 4 AgAtomen die beiden Gitter wieder überlappen [59]. Die Gitterfehlpassung beträgt in diesem Fall nur noch 0,3\%. Abbildung 4-13 zeigt ein maßstabsgetreues Modell der $\mathrm{Si}(100)$-Oberfläche mit darauf wachsender Ag- und $\mathrm{Cu}-\mathrm{Schicht}$ (ohne Durchmischung).

Das auf dem Ag deponierte $\mathrm{Cu}$ wächst wiederum $[100]_{\mathrm{Cu}} \|[100]_{\mathrm{Ag}}$ (Abbildung 4-11 unten), mit einer Halbwertsbreite der in-plane-Textur von $3,8^{\circ}$, obwohl die Gitterkonstante von $\mathrm{Cu}$ mit 0,361 nm um 13\% kleiner ist als die des Ag.

Das zugehörige Schichtpaket zeigt, daß die Epitaxie der Schichten nicht vollständig verloren geht (Abbildung 4-11 oben). Die (111)-orientierten Schichten wachsen vielmehr in vier diskreten Orientierungen auf der (100)-Oberfläche auf, wobei $[110]_{\mathrm{Cu}}\left\|[110]_{\mathrm{Ag}}\right\|[110]_{\mathrm{Si}}$. Dies führt zu der beobachteten zwölfzähligen Symmetrie, 


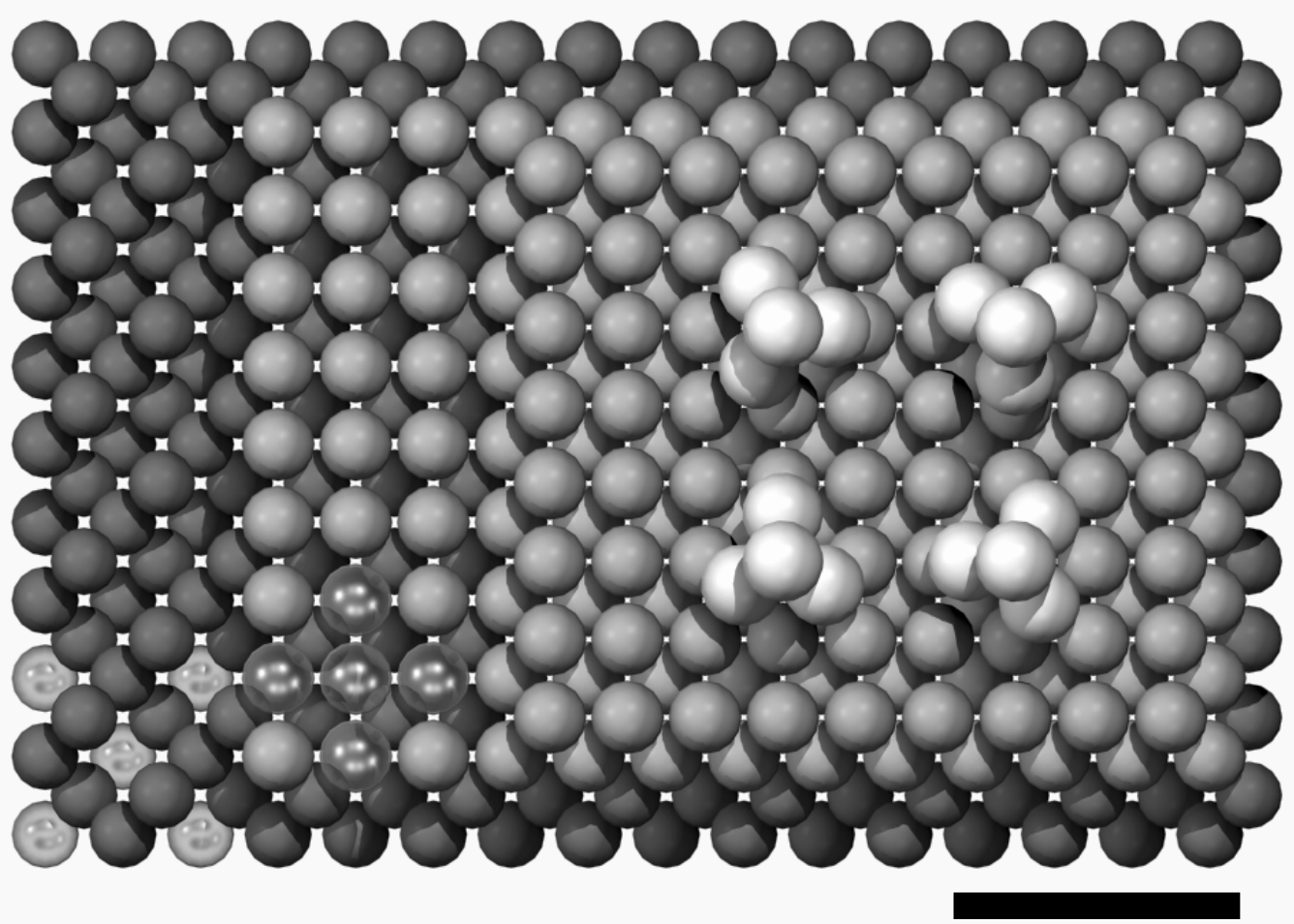

$1 \mathrm{~nm}$

Abbildung 4-14: Si-Cu-Ag-Schichtfolge im Modell harter Kugeln; dunkelgrau Si, hellgrau $\mathrm{Cu}$, weiß $\mathrm{Ag}$; für $\mathrm{Si}$ und $\mathrm{Cu}$ ist die Grundfläche der Einheitszelle mittels durchsichtiger Kugeln veranschaulicht

mit einer mittleren Halbwertsbreite der in-plane-Textur der $\mathrm{Cu}$ - und Ag-Einzellagen von jeweils $9,5^{\circ}$.

Abbildung 4-12 zeigt die gemessene Orientierungsbeziehung für die umgekehrte Schichtfolge Si-Cu-Ag und das entsprechende Schichtpaket. Auch hier wächst das $\mathrm{Cu}$ in (100)-Orientierung auf, allerdings um $45^{\circ}$ gedreht, d.h. $[100]_{\mathrm{Cu}} \|[110]_{\mathrm{Si}}$. Abbildung 4-14 zeigt dieses Wachstum im Modell harter Kugeln. Das Cu-Gitter ist erkennbar kleiner als das Si-Gitter.

Das auf dem $\mathrm{Cu}$ aufwachsende (111)-orientierte $\mathrm{Ag}$ zeigt die oben beschriebene zwölfzählige Symmetrie. Die Halbwertsbreite der in-plane-Textur ist in diesem Fall

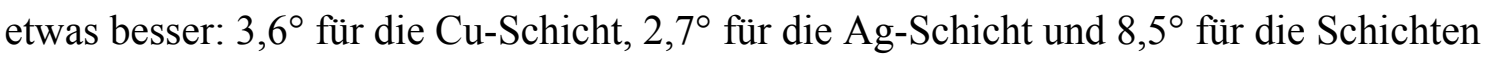
im Schichtpaket. Aus der um $45^{\circ}$ gedreht auf der Si(100)-Oberfläche aufgewachsenen $\mathrm{Cu}$-Schicht folgt, daß auch das Schichtpaket eine $45^{\circ}$-Drehung zur Orientierung der SiOberfläche aufweist, so daß hier im Unterschied zum obigen Schichtpaket die Orientierungsbeziehung $[110]_{\mathrm{Ag}}\left\|[110]_{\mathrm{Cu}}\right\|[100]_{\mathrm{Si}}$ lautet. 


\subsection{Mößbauerspektroskopie}

Die Mößbauerspektroskopie bietet die Möglichkeit, die nächsten atomaren Nachbarn in der Umgebung von ${ }^{57} \mathrm{Fe}$-Atomen in einer Probe zu untersuchen und ist damit nur auf den Anteil von Fremdatomen in einer ${ }^{57} \mathrm{Fe}$-Schicht empfindlich. Eine morphologische Rauhigkeit bei scharfer Grenzfläche ändert dagegen die Anteile der nächsten Nachbarn der ${ }^{57} \mathrm{Fe}$-Atome nicht. ${ }^{10}$ Die Mößbauerspektroskopie ermöglicht es somit im Zusammenhang mit den schon beschriebenen experimentellen Methoden, im System Fe-Ag die reine Durchmischung der Grenzfläche zu bestimmen.

\subsection{1 ${ }^{57} \mathrm{Fe}-\mathrm{Ag}-$ Schichtpakete}

Untersucht wurden zunächst zwei ${ }^{57} \mathrm{Fe}$-Ag-Schichtpakete mit Doppelschichtdicken $\Lambda$ von 2,3 nm (40 Doppelschichten) und 3,8 nm (20 Doppelschichten), wobei die ${ }^{57} \mathrm{Fe}-$ und die Ag-Schicht jeweils gleich dick sind. Abbildung 4-15a zeigt das Mößbauerspektrum der Schicht mit der Periode von 3,8 nm und Abbildung 4-15b das der Schicht mit 2,3 nm Periode. Es ist die für $\alpha$-Fe typische Aufspaltung des Übergangs in sechs Linien zu erkennen. Die asymmetrische Verbreiterung der Linien in Richtung betragsmäßig niedrigerer Geschwindigkeit läßt darauf schließen, daß neben ${ }^{57} \mathrm{Fe}$ mit einer reinen Fe-Umgebung auch von Ag-Atomen umgebenes ${ }^{57} \mathrm{Fe}$ zum CEMS-Signal beiträgt, da die Stärke der Aufspaltung abhängig ist von der Zahl der Ag-Atome, die als nächste Nachbarn der ${ }^{57} \mathrm{Fe}$-Atome auftreten. Mit Zunahme der Ag-Konzentration in der nächsten Nachbarschaft um ein ${ }^{57}$ Fe-Atom sinkt das durch die anderen Fe-Atome erzeugte magnetische Hyperfeinfeld $B_{h f}$ am Ort des ${ }^{57}$ Fe-Atoms linear ab [61,62], bis ab einem kritischen Anteil Ag im Fe die Kristallstruktur in fcc umschlägt und keine ferromagnetische Ordnung mehr existiert. In diesem Fall wird im Spektrum nur das durch die Quadrupolaufspaltung hervorgerufene Dublett beobachtet [63].

\footnotetext{
${ }^{10}$ Nur die Zahl der ${ }^{57} \mathrm{Fe}$-Atome direkt an der Grenzfläche nimmt mit der Rauhigkeit zu, da sich die Fläche der Grenzfläche minimal vergrößert. Dieser Effekt ist aber nicht meßbar, da einerseits die vertikale Größe der Rauhigkeit i. a. klein zu ihrer lateralen Ausdehnung ist und andererseits bei ${ }^{57} \mathrm{Fe}$-Schichten, die mehr als nur wenige Monolagen dick sind, der Anteil der ${ }^{57} \mathrm{Fe}$-Atome an der Grenzfläche klein zur Gesamtzahl der ${ }^{57} \mathrm{Fe}$-Atome ist.
} 
a)
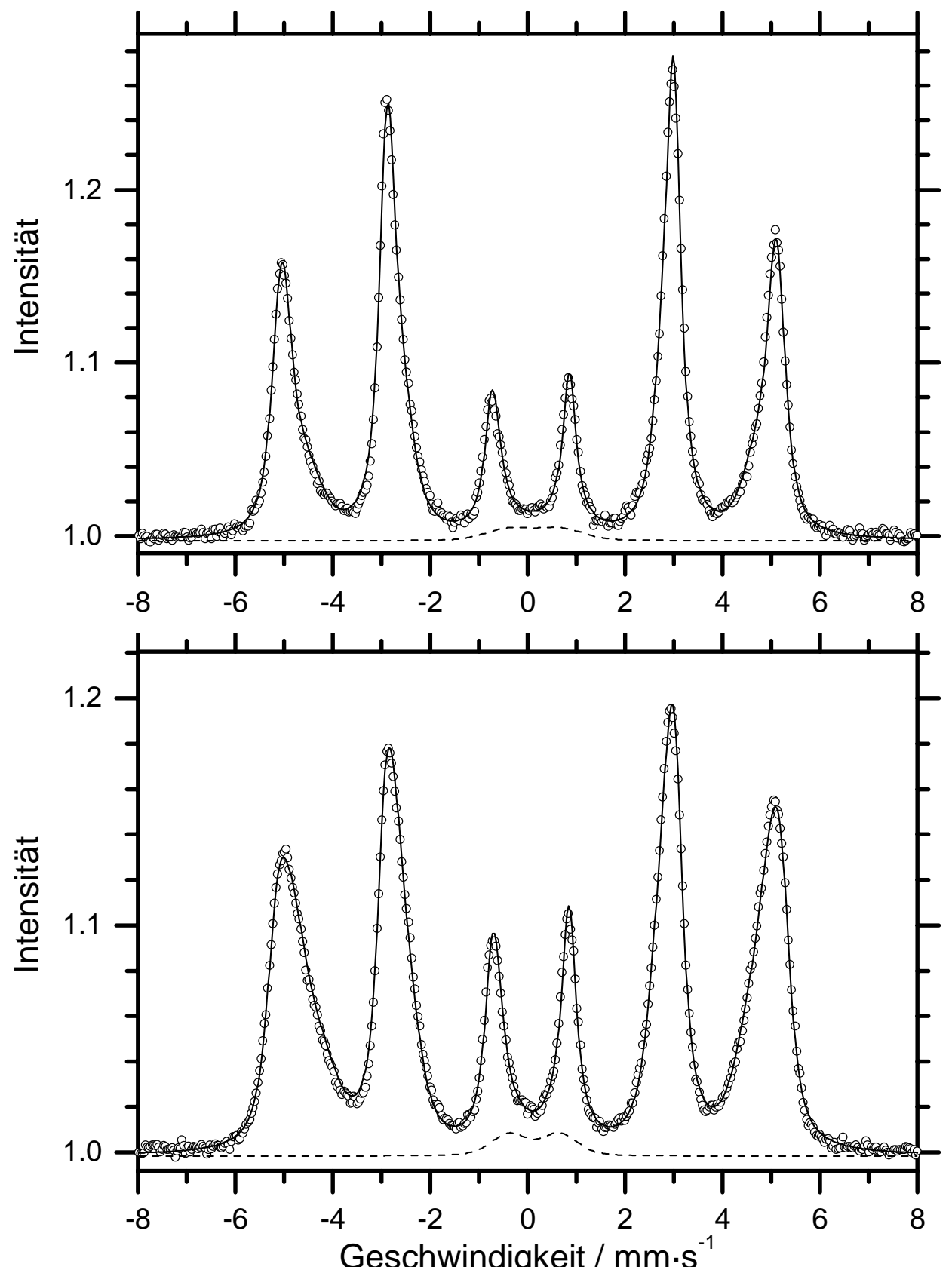

b)

Geschwindigkeit / $\mathrm{mm} \cdot \mathrm{s}^{-1}$

Abbildung 4-15: CEMS-Spektren von ${ }^{57} \mathrm{Fe}$-Ag-Schichtpaketen mit einer Doppelschichtdicke $\Lambda=3,8 \mathrm{~nm}$ (a) und $\Lambda=2,3 \mathrm{~nm}(b)$; durchgezogene Linie: bester Fit, gestrichelt: Dublett-Teilspektrum (siehe Text)

Ein Mößbauerspektrum läßt sich somit im allgemeinen Fall als eine Kombination diskreter Teilspektren mit verschiedenen Anteilen auffassen: Ein von der elektrischen Quadrupolaufspaltung herrührender Dublettanteil D und mehrere durch die magnetische Hyperfeinfeldaufspaltung hervorgerufene sechszählige Teilspektren. Bei den magnetischen Teilspektren wird im folgenden der Anteil, der durch eine reine Fe- 
Umgebung eines ${ }^{57} \mathrm{Fe}$-Atoms hervorgerufen wird, mit $\mathrm{M}_{0}$ bezeichnet. In diesem Fall wird das höchste Hyperfeinfeld von etwa $32 \mathrm{~T}$ und damit die stärkste Aufspaltung der Linien im Spektrum beobachtet. Wird eines der acht Fe-Atome in der nächsten Nachbarschaft des ${ }^{57} \mathrm{Fe}$ durch ein Ag-Atom ersetzt, verringert sich $B_{h f}$ auf etwa 30,5 T und es wird ein Spektrum $M_{1}$ mit entsprechend geringerer Aufspaltung beobachtet. Unter $\mathrm{M}_{2}$ werden schließlich alle Spektren zusammengefaßt, die durch die magnetische Hyperfeinfeldaufspaltung (etwa $28 \mathrm{~T}$ ) bei Vorhandensein von zwei oder mehr AgAtomen in der nächsten Umgebung hervorgerufen werden.

Durch Variation der Anteile D, $\mathrm{M}_{0}, \mathrm{M}_{1}$ und $\mathrm{M}_{2}$ wird ein Summenspektrum berechnet, welches die bestmögliche Übereinstimmung mit dem gemessenen Spektrum bietet [64]. In Abbildung 4-15 sind die gemessenen Spektren zusammen mit dem zugehörigen besten Fit dargestellt. Neben dem berechneten Summenspektrum ist das DublettTeilspektrum D aufgetragen. Dieses unterscheidet sich deutlich in beiden Fällen. Für das Schichtpaket mit der größeren Periode ist der D-Anteil kleiner, was auf den geringeren Einfluß des Durchmischungsbereiches der Grenzflächen (wo Fe viele AgAtome als nächste Nachbarn hat) zurückzuführen ist.

Da das magnetische Hyperfeinfeld am Ort des ${ }^{57} \mathrm{Fe}$-Atoms - und damit die Stärke der Aufspaltung der Linien im Spektrum - nicht nur von der Zahl der Ag-Atome in dessen nächster Umgebung, sondern auch in geringem Maße von den Atomen der übernächsten Schale und möglicherweise vorhandenen Gitterverzerrungen abhängt, wurde der zu den Konfigurationen $\mathrm{M}_{0}, \mathrm{M}_{1}$ und $\mathrm{M}_{2}$ gehörige Wert des Hyperfeinfeldes variiert, bis die bestmögliche Übereinstimmung mit dem gemessenen Spektrum erzielt wurde. Im Fall des Schichtpaketes mit $2,3 \mathrm{~nm}$ Periode konnten die Felder der $\mathrm{M}_{1}$ - und $\mathrm{M}_{2}$ Konfiguration nicht getrennt werden, weshalb sie in einem gemeinsamen Anteil mit gleichem Hyperfeinfeld zusammengefaßt wurden.

Die Anteile der so ermittelten Felder sind Abbildung 4-16 dargestellt. In beiden Fällen werden neben dem Beitrag des reinen $\mathrm{Fe}\left(\mathrm{M}_{0}\right.$-Anteil) auch Anteile von $\mathrm{M}_{1}$ und $\mathrm{M}_{2}$ und damit eine Durchmischung beobachtet. Für das Schichtpaket mit der kleineren Periode von 2,3 nm ist erwartungsgemäß ein höherer Anteil bei kleineren Feldstärken (d. h. mehr Ag-Atome als nächste Nachbarn des Fe) zu verzeichnen, da hier der Beitrag des durchmischten Bereiches an den Grenzflächen stärker ist. 


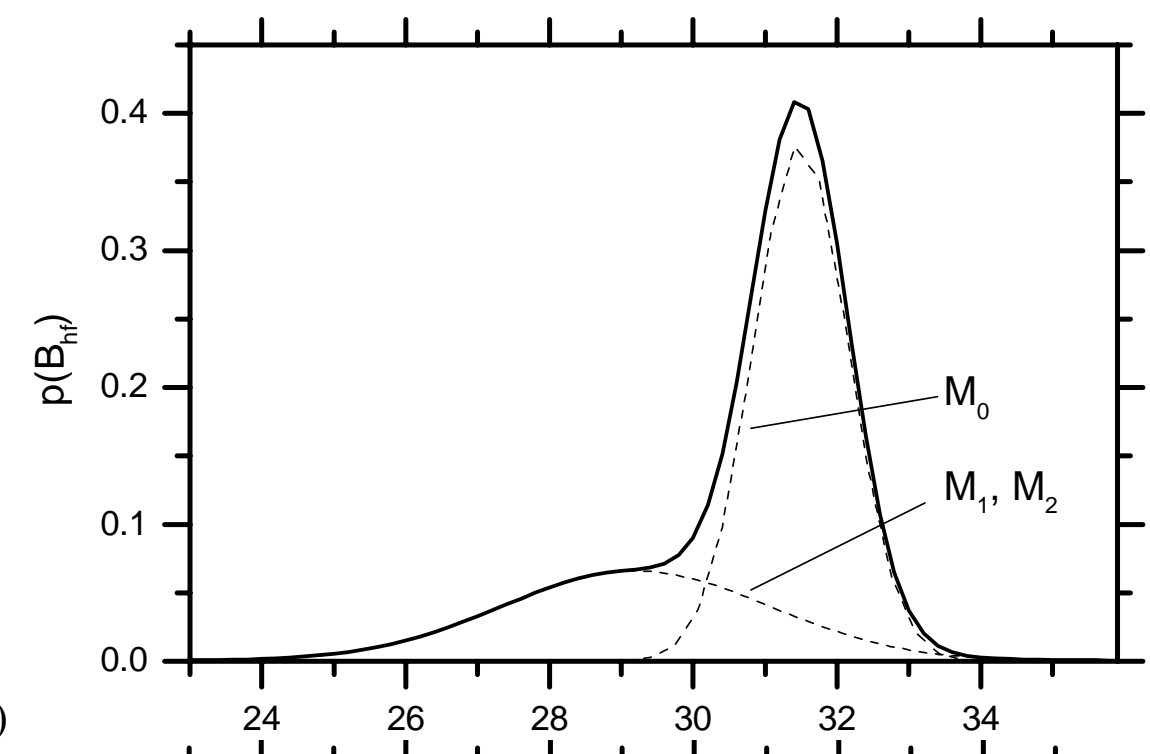

)

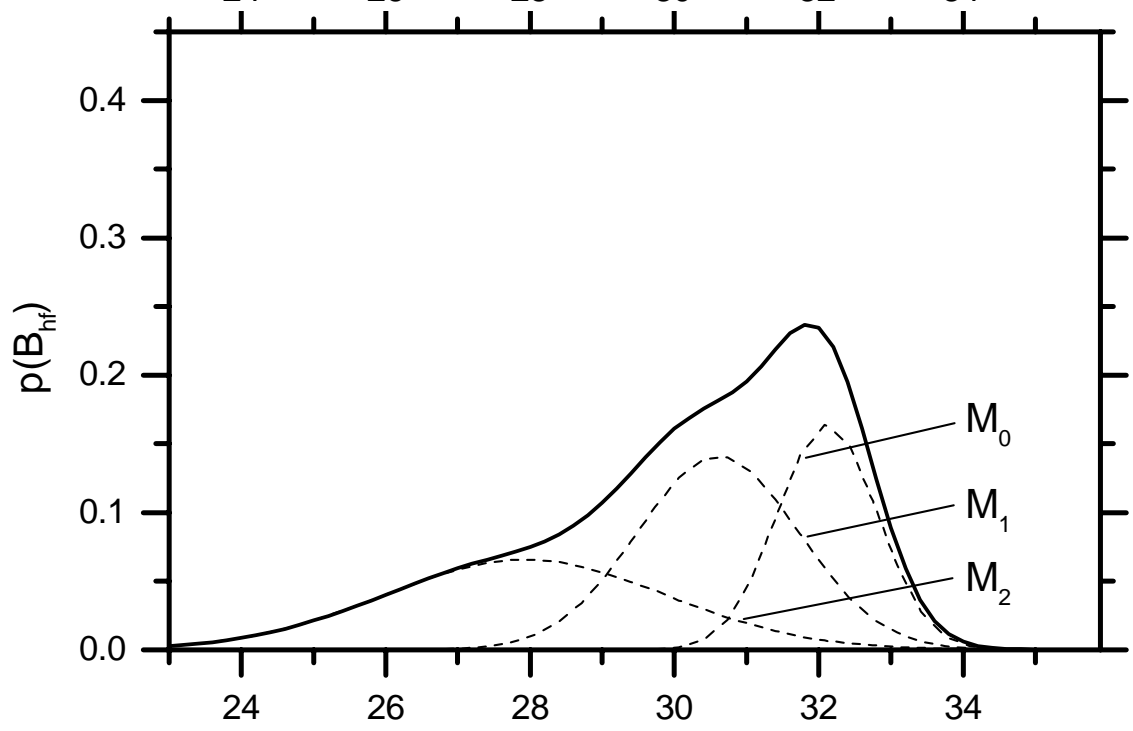

b)

Hyperfeinfeld $\mathrm{B}_{\mathrm{hf}} / \mathrm{T}$

Abbildung 4-16: Verteilung der Feldstärken des magnetischen Hyperfeinfeldes für a) 3,8 nm Doppelschichtdicke und b) 2,3 nm Doppelschichtdicke; Gestrichelt dargestellt sind die Anteile der Komponenten $M_{0}, M_{1}$ und $M_{2}$ 


\subsubsection{Fe-Ag-Schichtpakete mit ${ }^{57} \mathrm{Fe}-$ Markerschichten}

Die bisher beschriebenen Untersuchungen an ${ }^{57} \mathrm{Fe}-\mathrm{Ag}$-Schichtpaketen erlauben nur die Bestimmung von Größen, die über die gesamte ${ }^{57} \mathrm{Fe}-\mathrm{Schich}$ gemittelt sind. Um eine ortsaufgelöste $^{11}$ Messung $\mathrm{zu}$ ermöglichen, wurden Fe-Ag-Schichtpakete mit natürlichem Fe präpariert, bei denen eine $0,5 \mathrm{~nm}$ dicke ${ }^{57} \mathrm{Fe}-$ Markerschicht an unterschiedlichen Stellen in den Fe-Schichten plaziert wurde (Abbildung 4-17). Die Doppelschichtdicke betrug in allen
Abbildung 4-17: Aufbau der FeAg-Schichtpakete mit ${ }^{57} \mathrm{Fe}$ Markerschichten

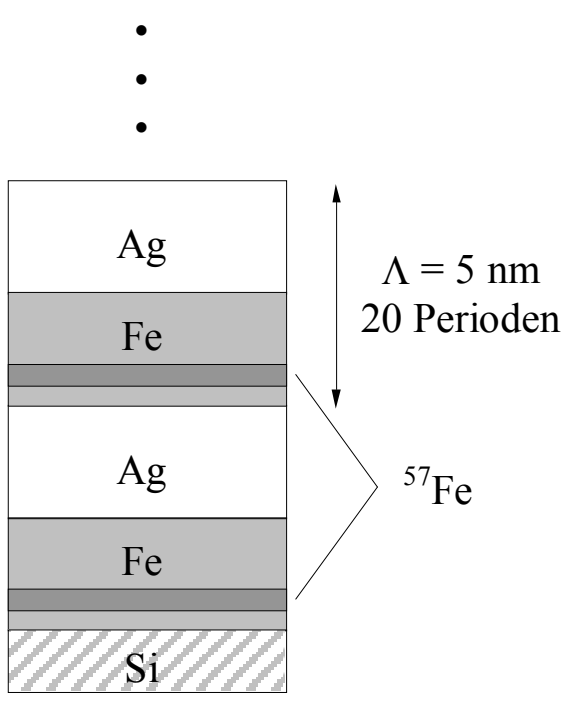

Schichtpaketen jeweils $0,5 \mathrm{~nm}$ weiter von der Fällen $5 \mathrm{~nm}$, mit gleichen Anteilen Fe und Ag, wobei die Markerschicht im ersten Fall direkt an der Ag-Fe-Grenzfläche und in den folgenden Grenzfläche entfernt deponiert wurde.

In natürlichem $\mathrm{Fe}$ beträgt der Isotopenanteil von ${ }^{57} \mathrm{Fe}$ nur 2,19\% [65], so daß die Intensität im Mößbauerspektrum hauptsächlich von den ${ }^{57} \mathrm{Fe}-\mathrm{Markerschichten} \mathrm{herrührt.}$ Auf diese Weise können Mößbauerspektren als Funktion des Abstandes von der Grenzfläche aufgenommen werden (Abbildung 4-18). Wie zuvor wurden die gemessenen Spektren mit einem Dublett-Spektrum D und drei sechszähligen Spektren $\mathrm{M}_{0}, \mathrm{M}_{1}$ und $\mathrm{M}_{2}$ angepaßt. Die Dublett-Anteile erwiesen sich dabei im Rahmen der Fehlergrenzen als gleich groß $(1,0-2,5 \%)$. Um eine quantitative Vergleichbarkeit der magnetischen Anteile bei allen Proben zu gewährleisten, wurden stets die selben Hyperfeinfelder verwendet: $31,7 \mathrm{~T}$ im Fall von $\mathrm{M}_{0}, 30,4 \mathrm{~T}$ für $\mathrm{M}_{1}$ und 28,4 $\mathrm{T}$ für $\mathrm{M}_{2}$. In Abbildung 4-19 sind die so bestimmten Anteile zusammen dargestellt. Es ist ein asymmetrischer Verlauf in allen drei Fällen zu beobachten. Der Anteil des reinen Fe $\left(\mathrm{M}_{0}\right)$ steigt schnell auf $75 \%$ bei einer Entfernung von $0,75 \mathrm{~nm}$ von der unteren, dem Substrat näheren Grenzfläche (Ag-Fe) an, um dann bis zur oberen Grenzfläche (Fe-Ag) wieder abzufallen. Dementsprechend verlaufen die auf $\mathrm{Ag}$ in der nächsten Nachbarschaft der ${ }^{57} \mathrm{Fe}$-Atome hinweisenden Anteile $\mathrm{M}_{1}$ und $\mathrm{M}_{2}$ entgegengesetzt.

\footnotetext{
${ }^{11}$ senkrecht zur Schicht, nicht lateral
} 


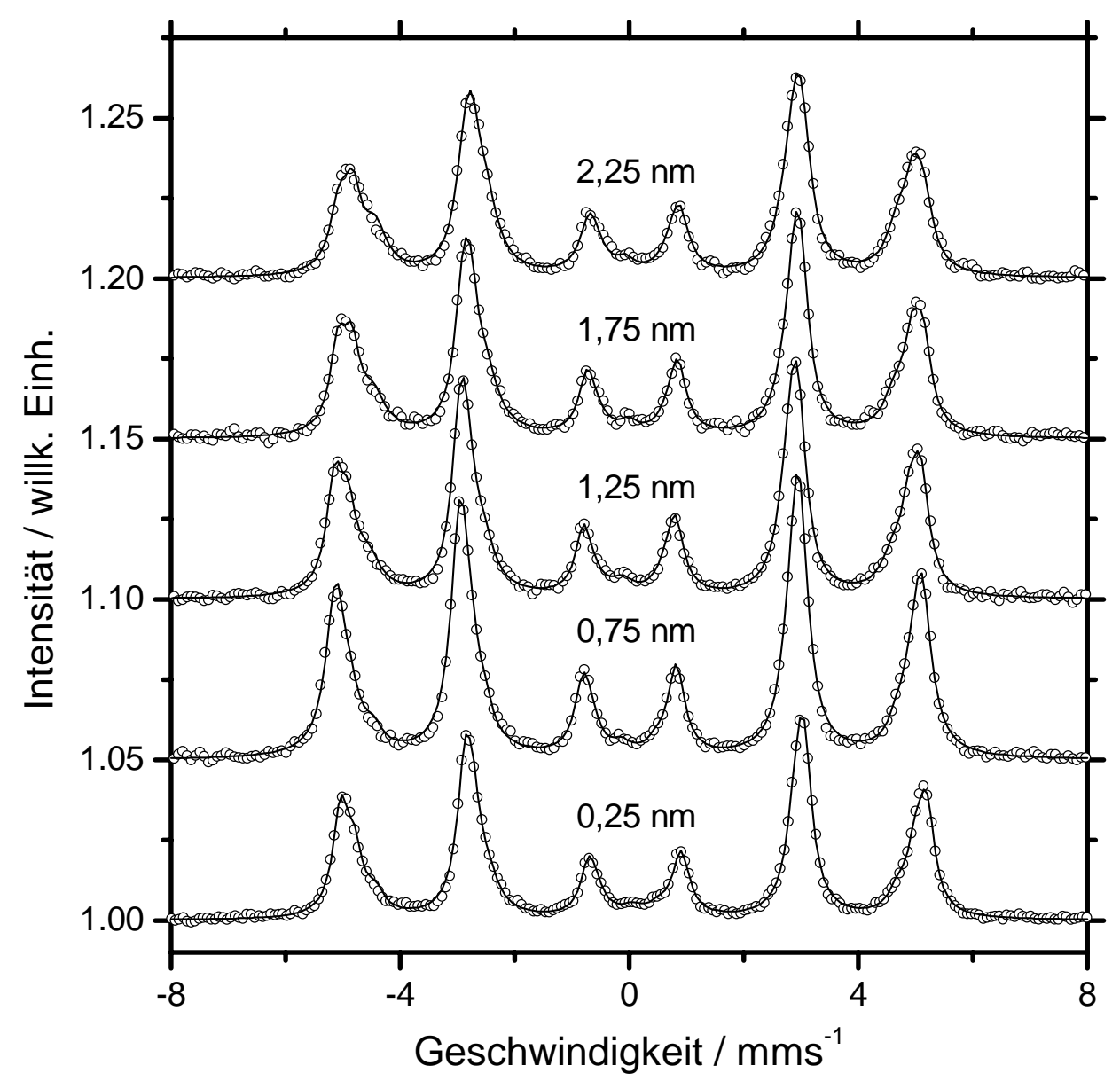

Abbildung 4-18: CEMS-Spektren der Fe-Ag-Schichtpakete (jeweils um 0,05 Einheiten versetzt aufgetragen) für die angegebenen mittleren Abstände der Markerschicht von der unteren Ag-Fe-Grenzfläche; offene Kreise: Messung, durchgezogene Linie: bester Fit

Setzt man eine statistische Verteilung der Ag-Atome im Fe voraus, kann man mit Hilfe der Binomialverteilung aus der Ag-Konzentration $c$ die Wahrscheinlichkeit $P(n, c)$ berechnen, genau $n$ Ag-Atome von den acht möglichen nächsten Nachbarn um ein ${ }^{57} \mathrm{Fe}$ Atom in der bcc-Kristallstruktur zu finden [66]:

$$
P(n, c)=\left(\begin{array}{l}
8 \\
n
\end{array}\right) c^{n}(1-c)^{8-n}
$$

Für den Fall $P(0, c)$, der der Konfiguration $\mathrm{M}_{0}$ entspricht, läßt sich (4-6) analytisch nach der Konzentration c auflösen:

$$
c=1-\sqrt[8]{P\left(M_{0}\right)}
$$




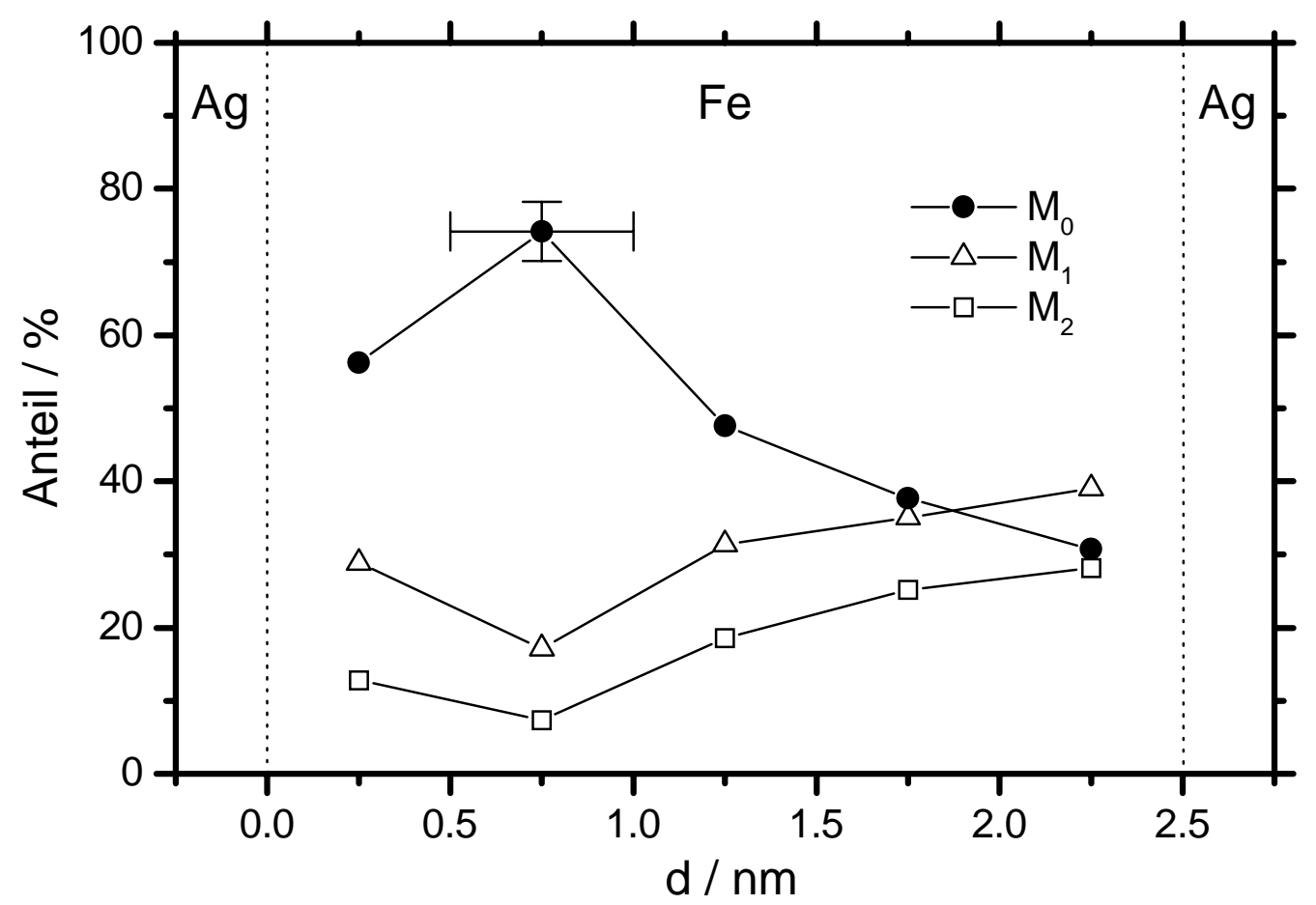

Abbildung 4-19: Anteile der Hyperfeinfelder $M_{0}$ bis $M_{2}$ in Abhängigkeit vom Abstand d der ${ }^{57} \mathrm{Fe}$-Markerschicht von der unteren Ag-Fe-Grenzfläche. Exemplarisch ist für einen Meßwert der für alle Messungen gültige Fehler eingezeichnet.

$P\left(\mathrm{M}_{0}\right)$ ist die Wahrscheinlichkeit, nur Fe-Atome als nächste Nachbarn eines ${ }^{57} \mathrm{Fe}$-Atoms $\mathrm{zu}$ finden und damit gleichbedeutend mit dem in Abbildung 4-19 dargestellten $\mathrm{M}_{0^{-}}$ Anteil. Die so berechneten Ag-Konzentrationen im bcc-Fe liegen zwischen 3 und 15\% (Abbildung 4-20).

Die idealisierte Voraussetzung einer statistischen Verteilung der Ag-Atome im Fe ist nur für kleine Ag-Konzentrationen erfüllt. Nach [16] sind bei Raumtemperatur laserdeponierte Fe-Ag-Legierungen ab etwa 16\% Ag entmischt. Diese Konzentration wird hier nur an der Fe-Ag-Grenzfläche erreicht, d. h. im Innern der Fe-Schicht kann eine Entmischung ausgeschlossen werden.

Aus der Lage des Minimums in Abbildung 4-20 läßt sich schließen, daß das Ag maximal bis zu 1,5 nm tief in das Fe implantiert wird. Dieses Ergebnis paßt gut zu der in [11] berechneten mittleren Eindringtiefe von $0,8 \mathrm{~nm}$. Auch das Fe sollte nach diesen Berechnungen ähnlich tief in das Ag implantiert werden. Statt dessen wird nur ein maximaler Durchmischungsbereich von $0,5 \mathrm{~nm}$ beobachtet. Offensichtlich diffundiert schon während der Deposition ein Teil des Fe wieder aus der Ag-Schicht heraus, so daß 


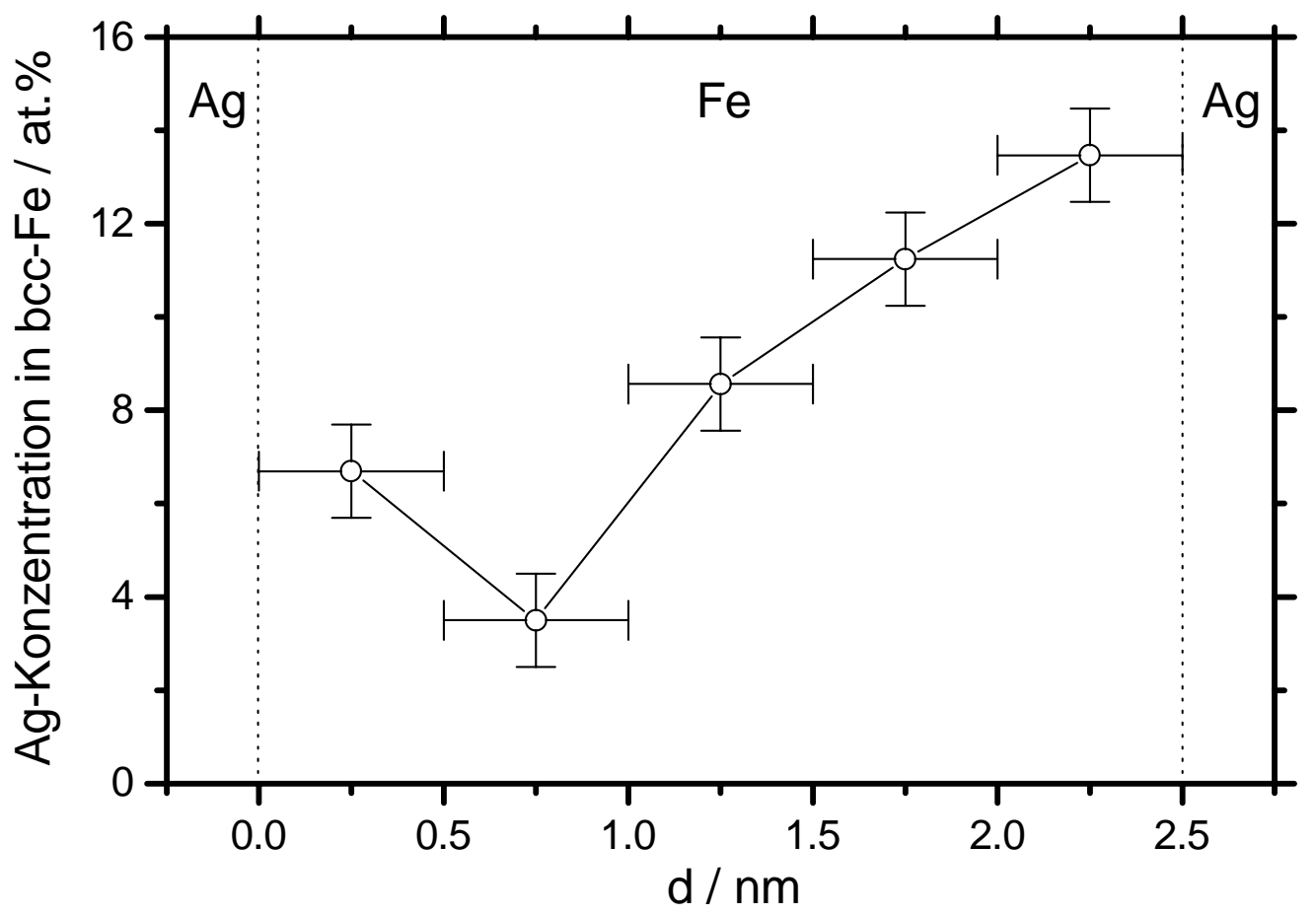

Abbildung 4-20: Aus dem Anteil des Hyperfeinfeldes $M_{0}$ (Abbildung 4-19) bestimmte Ag-Konzentration im Fe

sich eine scharfe Grenzfläche ausbilden kann. Diese Interpretation steht im Einklang mit Messungen von M. Störmer, die eine verschwindend kleine Mischbarkeit von bei Raumtemperatur deponiertem Fe in Ag zeigen [52,16].

Zusätzlich zur Entmischung der Ag-Fe-Grenzfläche führt das in Kapitel 3.2.2 als Begründung für den steilen Anstieg der Fe-Oberflächenkonzentration angeführte starke Sputtern der Ag-Atome zu einer Verkleinerung der Grenzflächenbreite und damit zu einem steileren Konzentrationsverlauf in der Grenzfläche. Allerdings spielt dieser Effekt in diesem Fall eine geringere Rolle, da sich der Konzentrationsgradient der Grenzfläche in einer Tiefe von bis zu etwa 1,5 nm (maximale Implantationstiefe der FeIonen) aufbaut, die durch den Abtrag von 2 bis 3 Monolagen der Ag-Oberfläche nur indirekt beeinflußt wird.

Aufgrund der Entmischung der Fe-Ag-Schichtpakete schon während der Deposition ist keine Übereinstimmung mit dem in Kapitel 3.3.2 beschriebenen rein ballistischen Depositionsmodell zu erwarten. Dieses führt zu einer stärkeren Durchmischung der Grenzfläche, wie sich anhand des simulierten Konzentrationsprofils in Abbildung 4-21 


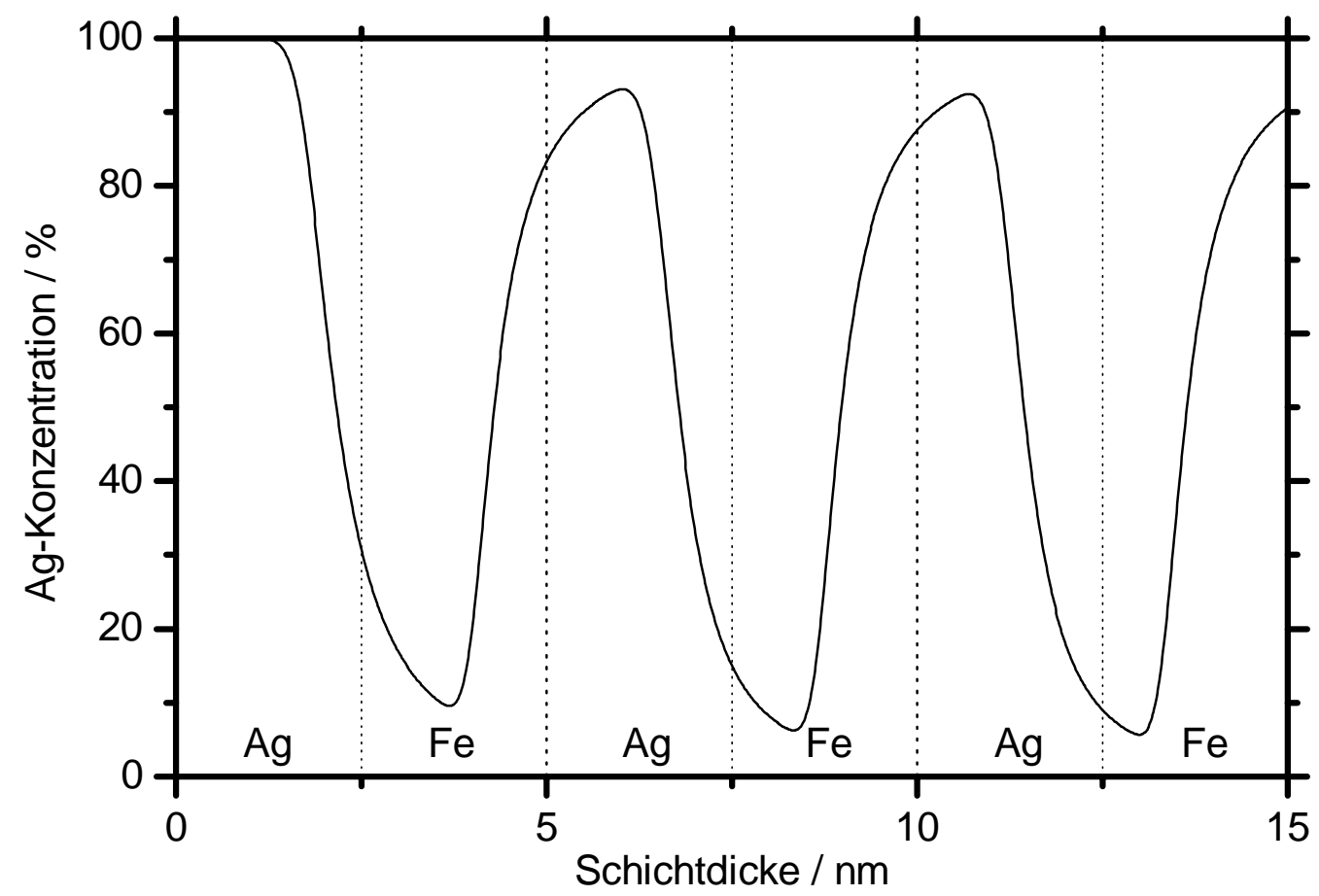

Abbildung 4-21: Simuliertes Konzentrationsprofil der Fe-Ag-Schichtpakete mit $5 \mathrm{~nm}$ Periode; Dargestellt sind nur die ersten drei Doppellagen. Gestrichelt sind die Bereiche markiert, in denen Ag bzw. Fe deponiert wurde.

erkennen läßt. Nur die minimale Ag-Konzentration im Fe von 5,5\% liegt im Bereich des gemessenen Wertes (3,5\%, Abbildung 4-20).

Werden Fe-Ag-Schichtpakete mit größerer Doppelschichtdicke laserdeponiert, sinkt die Ag-Konzentration im Innern der Fe-Schicht erwartungsgemäß ab. Dies wurde durch die Deposition einer ${ }^{57} \mathrm{Fe}-$ Markerschicht in die Mitte der Fe-Einzelschichten für Schichtpakete mit einer Periode von 10, 20 und $50 \mathrm{~nm}$ überprüft (Gesamtschichtdicke jeweils $100 \mathrm{~nm}$ ). Bei einer Doppelschichtdicke von $10 \mathrm{~nm}$ beträgt die Ag-Konzentration in der Mitte der $5 \mathrm{~nm}$ dicken Fe-Schicht nur noch 1,7\%, während für $20 \mathrm{~nm}$ und $50 \mathrm{~nm}$ im Rahmen der Auflösung kein Ag mehr nachweisbar ist.

\subsubsection{Ratenabhängigkeit der Grenzflächendurchmischung}

Um zu untersuchen, ob die Depositionsrate einen Einfluß auf die Grenzflächendurchmischung hat, wurden Fe-Ag-Schichtpakete aus 20 Doppelschichten Fe-Ag mit einer Doppelschichtdicke von $5 \mathrm{~nm}$ bei verschiedenen Target-SubstratAbständen deponiert. Die Einzelschichtdicke der Fe- und Ag-Schichten war dabei mit 


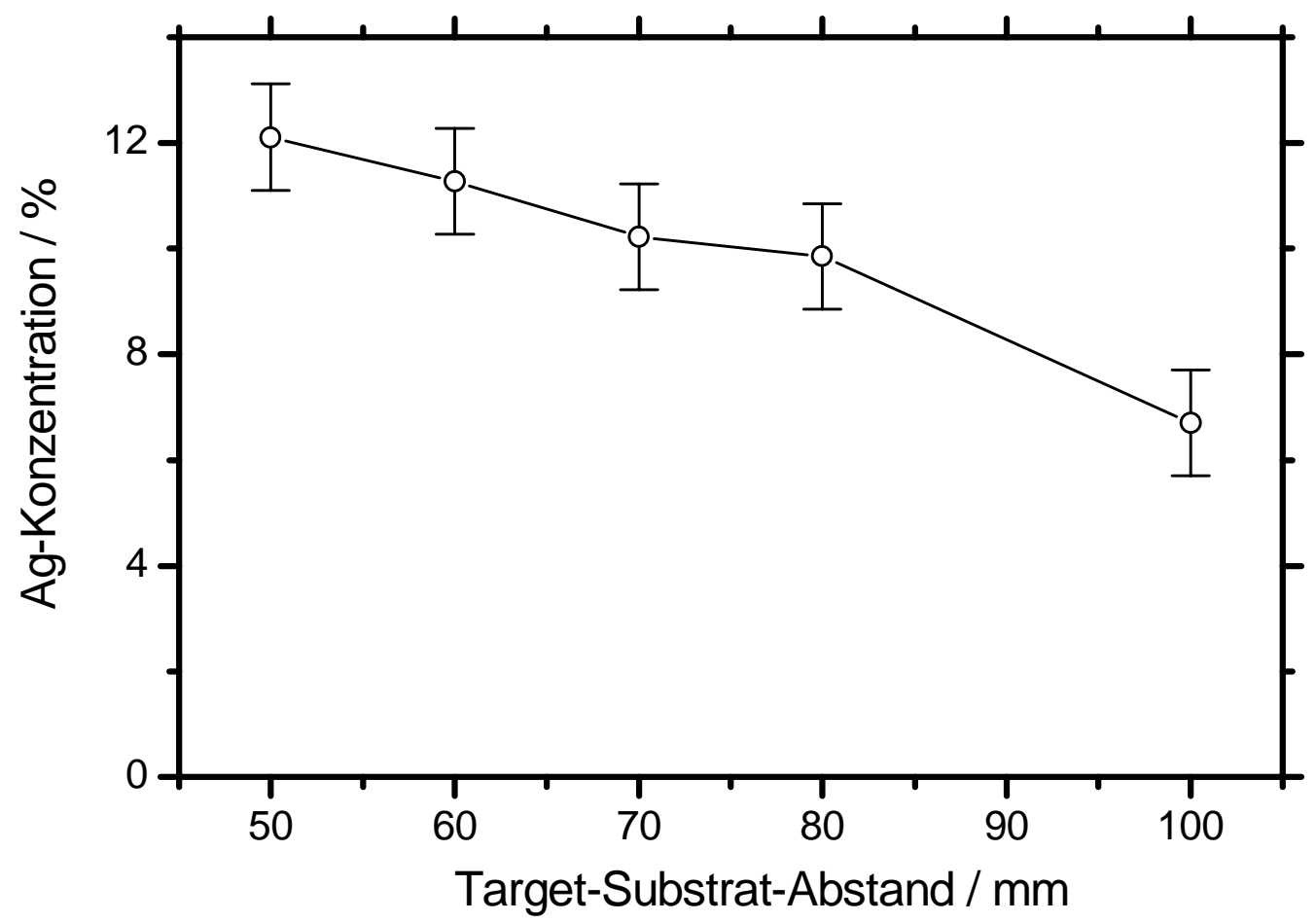

Abbildung 4-22: Ag-Konzentration in der Mitte der Fe-Schichten in Abhängigkeit vom Abstand zwischen Target und Substrat

$2,5 \mathrm{~nm}$ stets gleich, wobei jeweils in die Mitte der Fe-Schichten eine ${ }^{57} \mathrm{Fe}$ Markerschicht eingefügt wurde.

Durch die Vergrößerung der Entfernung des Substrates kann eine definierte Verringerung der Depositionsrate entsprechend dem annähernd quadratischen Abstandsgesetz (2-1) erzielt werden. Für Target-Substrat-Abstände im Zentimeterbereich und darüber ist die Geschwindigkeitsverteilung der Teilchen im Laserplasma konstant, da Stöße der Teilchen untereinander nur bis zu einer Entfernung von einigen Millimetern von der Targetoberfläche stattfinden (innerhalb der sog. Knudsenlage). Für größere Abstände wird ein freier Flug der Teilchen ohne weitere Stöße beobachtet [8]. Damit sind außer der Depositionsrate alle Parameter insbesondere die kinetische Energie der Ionen - konstant.

Auch durch die Variation der Laserenergiedichte oder der Laserpulsfrequenz läßt sich die Depositionsrate verändern. Im ersten Fall ändert sich allerdings zusätzlich die Energie der Ionen und ihr Anteil im Laserplasma, während mit der Änderung der 
Pulsfrequenz zwar die mittlere Depositionsrate eingestellt werden kann, die momentane Rate pro Puls jedoch unverändert ist.

Die Auswertung der CEMS-Spektren erfolgte wie in Kapitel 4.2.2 beschrieben. Die aus den $\mathrm{M}_{0}$-Anteilen bestimmten Ag-Konzentrationen sind in Abbildung 4-22 als Funktion des Target-Substrat-Abstandes bei der Herstellung dargestellt. Es ist ein Abfall der AgKonzentration mit steigendem Abstand, d.h. mit sinkender Depositionsrate zu erkennen. Da Wiederabtrag und Implantation aufgrund der konstanten Energie der Ionen für alle Abstände gleich sind, muß die Kinetik der Entmischung durch die veränderte Depositionsrate beeinflußt sein. Für die genaue Art des Einflusses der Rate auf die Entmischungskinetik bieten sich zwei Möglichkeiten an:

Einerseits ist die Entmischung ein mit dem Schichtwachstum konkurrierender Prozeß. Je schneller die Schicht wächst, desto weiter ist der Weg, den die implantierten Teilchen bis zur Oberfläche zurücklegen müssen. Bei hohen Wachstumsgeschwindigkeiten werden die implantierten Teilchen im Volumen „eingefroren“, da die Bulk-Diffusion deutlich kleiner sein kann als eine Diffusion in oberflächennahen Bereichen (wenige Monolagen tief), wie Molekulardynamiksimulationen von metallischen Gläsern zeigen $[67,68]$.

Eine zweite Möglichkeit besteht darin, daß ein unter der Oberfläche diffundierendes Atom durch ein implantiertes Ion wieder tiefer in die Schicht gestoßen wird. Da pro Puls typischerweise nur einige Prozent einer Monolage deponiert werden, ist ein solches Stoßereignis erst nach einigen zehn Pulsen (d. h. nach einigen Sekunden bei einer Repetitionsrate von $10 \mathrm{~Hz}$ ) zu erwarten, was eine entsprechend langsame Diffusion voraussetzt.

Das oft diskutierte thermal-spike-Modell [69,70], welches eine sehr kurze (wenige Picosekunden) und lokal begrenzte (wenige Nanometer) Aufschmelzung um den Implantationsort eines Teilchens mit entsprechend starker Diffusion in diesem Bereich beschreibt - selbst bei Energien von nur $100 \mathrm{eV}$ [71,72] - kann die hier beobachtete Abhängigkeit zwischen Rate und Durchmischung nicht erklären. Für eine mittlere Energie von $100 \mathrm{eV}$, die bei der Laserdeposition pro implantiertem Ion in das Material eingebracht wird, läßt sich bei gleichförmiger Energieverteilung ein oberer Grenzwert für das aufgeschmolzene Volumen (thermische Energie pro Atom um 0,1 eV) von etwa 1000 Atomen abschätzen. Damit wäre zwar eine räumliche Überlappung einzelner thermal spikes während eines Pulses denkbar, allerdings ist die Zeit für die 
Rekristallisation des thermal-spike-Bereiches (einige ps, [73] ${ }^{12}$ ) mehrere Größenordnungen kleiner als die Zeit, während der das Material eines Pulses deponiert wird (einige $\mu \mathrm{s},[74]$ ), so daß eine zeitliche Überlappung ausgeschlossen werden kann und die einzelnen Bereiche unabhängig von der Depositionsrate konstante Bedingungen für die Diffusion bieten.

Zusammenfassend läßt sich über die Mößbauerspektroskopie sagen, daß sie in Kombination mit ${ }^{57} \mathrm{Fe}-$ Markerschichten die Bestimmung des Konzentrationsverlaufes in Fe-Ag-Grenzflächen ermöglicht und dabei eine starke Asymmetrie sowie eine Ratenabhängigkeit der Durchmischung der Grenzflächen aufgezeigt hat. Mit den hauptsächlich für die ex-situ-Untersuchung der $\mathrm{Cu}$-Ag-Schichtsysteme eingesetzten Methoden Röntgendiffraktometrie und Kleinwinkel-Röntgenstreuung konnten die Epitaxiebeziehungen zwischen $\mathrm{Si}, \mathrm{Cu}$ und $\mathrm{Ag}$ sowie die Rauhigkeit bzw. Durchmischung der inneren Grenzflächen bestimmt werden. Die Messung an Einzelschichten verschiedener Dicke ergab eine erhöhte Dichte der $\mathrm{Cu}$ - und AgSchichten und erlaubte die Bestimmung der Oberflächenrauhigkeit in Abhängigkeit von der Schichtdicke. Dies ermöglicht im Vergleich mit der Ausdehnung der inneren Grenzflächen eine Trennung in Grenzflächenrauhigkeit und -durchmischung.

\footnotetext{
12 Diese Zeit wurde über die Molekulardynamiksimulation eines thermal spikes, der durch ein Atom mit $10 \mathrm{keV}$ Primärenergie erzeugt wurde, bestimmt. Da bei der Laserdeposition die auftretenden Energien deutlich geringer sind, ist dies nur eine grobe obere Abschätzung.
} 


\section{Vergleichende Diskussion der Ergebnisse}

Die in den vorangegangenen Kapiteln beschriebenen Ergebnisse sollen im folgenden zusammenhängend diskutiert werden. Aus den sich ergänzenden Aussagen der in-situund ex-situ-Experimente wird ein Bild für das Wachstum von $\mathrm{Cu}-\mathrm{Ag}$ - und Fe-AgSchichtsystemen auf Si-Substraten und die Entstehung von Grenzflächenrauhigkeit und -durchmischung gewonnen. Dabei wird sich zeigen, daß die charakteristischen Eigenschaften der gepulsten Laserdeposition, insbesondere die hohe Energie der deponierten Teilchen, einen entscheidenden Einfluß auf das Wachstum der Schichten und den Aufbau der Grenzflächen haben.

\subsection{Cu-Ag-Schichtsysteme}

\subsubsection{Cu auf $\mathrm{Si}(100)$}

Bei der Deposition von $\mathrm{Cu}$ oder Ag auf Si(100) unter UHV-Bedingungen tragen die energetischen Ionen zunächst die amorphe $\mathrm{SiO}_{2}$-Schicht auf dem Si-Substrat ab. Dies kann direkt aus dem epitaktischen Wachstum der $\mathrm{Cu}$ - und $\mathrm{Ag}$-Schichten gefolgert werden, da bei Vorhandensein einer amorphen Zwischenschicht eine Epitaxie zwischen Substrat und deponierter Schicht nicht möglich wäre. Das Sputtern von Oberflächenverunreinigungen, wie Oxidschichten und Kohlenwasserstoffe, mit Hilfe von elektrostatisch beschleunigten Eigenionen beim Aufdampfen von $\mathrm{Cu}$ ist von $\mathrm{P}$. Bai et al. mittels Sekundärionenmassenspektroskopie (SIMS) nachgewiesen und theoretisch beschrieben worden [75].

Das bei Raumtemperatur stattfindende epitaktische Wachstum auf Si-Substraten mit natürlicher Oxidschicht ist eine bemerkenswerte Eigenschaft der PLD, welche bei der Schichtpräparation durch Sputtern oder Aufdampfen nicht beobachtet wird. Um Epitaxie auf Si mit diesen Depositionsmethoden zu erreichen, muß die $\mathrm{SiO}_{2}$-Schicht auf dem Si-Substrat zuvor mit verdünnter Flußsäure entfernt werden, wobei die $\mathrm{H}^{+}$-Ionen der Säure die freien Bindungen an der Si-Oberfläche absättigen und auf diese Weise die Oberfläche für einige Stunden vor Oxidation schützen [76,77,59]. Alternativ können eine Oxidschicht und andere Verunreinigungen auch in-situ durch Aufheizen des Substrates über $800{ }^{\circ} \mathrm{C}$ [78] oder mit Hilfe eines Ionenstrahls entfernt werden [79]. 
Röntgendiffraktometrie an einer auf $\mathrm{Si}(100)$ laserdeponierten $50 \mathrm{~nm}$ dicken $\mathrm{Cu}-\mathrm{Schicht}$ zeigt eine in-plane-Textur von 3,6 $6^{\circ}$, wobei die Einheitszelle des $\mathrm{Cu}$ um $45^{\circ}$ gedreht zur Si-Zelle (d. h. Si[100] || Cu[110]) aufwächst (Abbildung 4-14). In dieser Orientierung wird die Gitterfehlpassung von $-33 \%$, die beim cube-on-cube-Wachstum zu erwarten wäre, auf $-5,9 \%$ reduziert. Trotz dieser noch recht hohen Fehlpassung wird diese Epitaxiebeziehung auch von anderen Gruppen und unter Verwendung anderer Depositionsmethoden bei niedrigen Substrattemperaturen $\left(\mathrm{T}<100{ }^{\circ} \mathrm{C}\right)$ beobachtet, insbesondere Aufdampfen [80-82] und Sputtern [78,83]. Die Ausbildung der guten Epitaxie zwischen $\mathrm{Cu}$-Schicht und Si-Substrat wird verschiedentlich mit der Bildung einer $\mathrm{Cu}_{3} \mathrm{Si}$-Silizidschicht an der Grenzfläche zwischen $\mathrm{Cu}$ und $\mathrm{Si}$ in Verbindung gebracht [82,84], welche die Grenzflächenenergie reduzieren soll. In [82] wird beispielsweise eine $10 \mathrm{~nm}$ dicke durchmischte Schicht beobachtet.

Diese Erklärung wird in anderen Veröffentlichungen bestritten [81,83,85], und auch in dieser Arbeit können keine Hinweise auf eine Silizidschicht mit solcher Dicke gefunden werden. Die Ergebnisse der Kleinwinkel-Röntgenstreuung zeigen, daß die Grenzfläche zwischen $\mathrm{Cu}$ und $\mathrm{Si}$ mit im Mittel 1,5 nm etwa $1 \mathrm{~nm}$ breiter als die ursprüngliche Rauhigkeit des Si-Substrates ist, was sich zwanglos durch die rein ballistische Implantation der schnellen $\mathrm{Cu}$-Ionen in die obersten Monolagen des Substrates erklären läßt. Mit einer thermodynamisch getriebenen Silizidbildung ist nach [86] erst ab Temperaturen von etwa $470 \mathrm{~K}$ zu rechnen.

Die Oberflächenrauhigkeit der laserdeponierten $\mathrm{Cu}$-Schichten ist sehr gering. Für dünne Schichten entspricht sie der Rauhigkeit des Substrates von 0,4 nm, während für eine $380 \mathrm{~nm}$ dicke Schicht 1,8 nm erreicht werden (Abbildung 4-4). Dagegen sind bei Raumtemperatur aufgedampfte Schichten deutlich rauher: Lukaszew et al. haben an $200 \mathrm{~nm}$ dicken auf $\mathrm{Si}(100)$ aufgedampften $\mathrm{Cu}$-Schichten Oberflächenrauhigkeiten von $7 \mathrm{~nm}$ gemessen [87]. Erst nach einer vierstündigen Auslagerung bei $420 \mathrm{~K}$ reduziert sich diese Rauhigkeit auf $1 \mathrm{~nm}$, was identisch ist mit dem für eine laserdeponierte $200 \mathrm{~nm}$ dicke $\mathrm{Cu}-\mathrm{Schicht}$ zu erwartenden Wert (Abbildung 4-4).

Die geringe Oberflächenrauhigkeit und die gute Textur sind als Folge der kinetischen Energie der deponierten Ionen zu verstehen. Karr et al. berichten in [71], daß sowohl Morphologie als auch Textur aufgedampfter Cu-Schichten auf Si(100) durch die Kodeposition von $\mathrm{Cu}$-Ionen deutlich verbessert werden können, wenn deren kinetische Energie oberhalb der Verlagerungsenergie von 25 eV [43] liegt. Dabei wurde von ihnen 
keine starke Abhängigkeit im untersuchten Energiebereich bis $100 \mathrm{eV}$ gefunden. Ein verbessertes Wachstum wird auch bei gleichzeitiger Bestrahlung mit Ar-Ionen beobachtet. Ionen von $500 \mathrm{eV}$ und einem Anteil von $80 \%$ an der Gesamtzahl der auf das Substrat treffenden Teilchen führen zu einem Umschlag des normalerweise auftretenden Inselwachstums ${ }^{13}$ von $\mathrm{Cu}$ auf $\mathrm{Si}(100)$ zu einem lagenweisen Wachstum [89].

Diese Beobachtungen können dadurch erklärt werden, daß die Ionen nach ihrem Eindringen in die obersten Monolagen in ihrer Mobilität eingeschränkt sind und nicht mehr durch Oberflächendiffusion ihre thermodynamisch günstigsten Positionen einnehmen können. Außerdem erzeugen die implantierten Ionen Defekte an der Oberfläche [90], die bei einem hohen Ionenanteil in entsprechend hoher Zahl vorliegen und als stationäre Keimzentren für die auf der Oberfläche deponierten niederenergetischen Atome dienen [89,91,92]. Auf diese Weise wird die Ausbildung von thermodynamisch bevorzugten großen Inseln verhindert und statt dessen eine Vielzahl von kleinen Inseln gebildet. Kleine Inseln fördern das Lagenwachstum, da die auf ihnen diffundierenden Atome einen kleineren mittleren Abstand zum Rand der Insel haben und somit mit höherer Wahrscheinlichkeit die sog. Ehrlich-Schwöbel-Barriere [93] überwinden und über den Rand der Insel springen können.

Neben diesem rein geometrischen Argument gibt es auch Hinweise darauf, daß die Ehrlich-Schwöbel-Barriere für kleine Inseln erniedrigt oder gar nicht vorhanden ist [94] und daß wachsende Inseln durch energiereiche Ionen fragmentiert werden können [91].

Bei der gepulsten Laserdeposition wirkt sich neben der kinetischen Energie der Ionen die sehr hohe momentane Rate während eines Pulses (fünf Größenordnungen höher als bei kontinuierlichen Depositionsverfahren) positiv auf eine hohe Inseldichte aus: Durch die innerhalb kürzester Zeit auf das Substrat treffenden Teilchen bilden sich schnell unbewegliche Cluster aus mehreren Atomen aus, die ebenfalls als stationäre Keimzentren anzusehen sind [95].

Durch die Implantation der schnellen Ionen werden außerdem Zwischengitteratome erzeugt, die die aufwachsende Schicht verdichten (das sog. shot peening [16]) und hohe Druckspannungen zur Folge haben. Dies führt dazu, daß die aufgrund der Gitterfehlpassung von $-6 \%$ beim epitaktischen Wachstum von $\mathrm{Cu}$ auf $\mathrm{Si}(100) \mathrm{zu}$ erwartenden Zugspannungen nicht auftreten, sondern überkompensiert werden - bei der

\footnotetext{
${ }^{13}$ Die Oberflächenenergie des $\mathrm{Cu}$ ist höher als die des Si [88], weshalb Inselwachstum zu erwarten ist.
} 
gepulsten Laserdeposition im Vakuum werden stets Druckspannungen beobachtet [57]. Die Verdichtungszone erstreckt sich nach T. Scharf etwa $3 \mathrm{~nm}$ in die aufwachsende Schicht hinein [57], was deutlich mehr ist als die mittlere Implantationstiefe von etwa $1 \mathrm{~nm}$. Da die implantierten Atome Stoßkaskaden auslösen können, welche Atome in tiefere Schichten verlagern, kann durchaus eine größere Verdichtungstiefe erreicht werden als die Implantationstiefe vermuten läßt.

Die Verdichtung des Films durch das shot peening verhindert auch die Ausbildung von sog. voids [96], die bei der Deposition mit Teilchen thermischer Energie auftreten können und zu einer Schicht mit geringerer Dichte führen [55,97]. Laserdeponierte $\mathrm{Cu}$ Schichten sind statt dessen bis zu einer Dicke von etwa $150 \mathrm{~nm}$ im Mittel 0,3\% dichter als $\mathrm{Cu}$ im Gleichgewicht (Abbildung 4-7). Setzt man eine homogene Verdichtung des Materials voraus, läßt sich über den Elastizitätsmodul des Cu von $130 \mathrm{GPa}$ daraus eine moderate Druckspannung von $130 \mathrm{MPa}$ ableiten. ${ }^{14}$ Dies ist vergleichbar mit den Druckspannungen, die von Y. H. Cheng et al. bei der Sputterdeposition von $\mathrm{Cu}$ mit einer Substratvorspannung zwischen 400 und 600 V beobachtet werden [98].

\subsubsection{Ag auf $\mathrm{Cu}(100)$}

Die Gitterkonstante des Ag ist mit 0,40853 nm um 13\% größer als die des $\mathrm{Cu}$. Bei Drehung des Ag-Gitters um 45', so daß $\mathrm{Cu}[110] \| \mathrm{Ag}[100]$ analog zum Wachstum von $\mathrm{Cu}$ auf $\mathrm{Si}$, sind die Bedingungen für epitaktisches (100)-Wachstum der Ag noch schlechter. Die Gitterfehlpassung beträgt in diesem Fall -20\%. Denkbar wäre die Ausbildung einer $9 \times 9$-Überstruktur, bei der $9 \times 9$ Ag-Gitterzellen auf $10 \times 10 \mathrm{Cu}$-Zellen kohärent aufwachsen und die Gitterfehlpassung auf 1,7\% reduziert ist. Molekulardynamiksimulationen zeigen jedoch, daß die Grenzflächenenergie einer solchen Struktur höher ist als die einer (111)-orientierten Ag-Schicht in einer 10×2Überstruktur [99], weshalb mit (111)-Wachstum zu rechnen ist. Dies wird auch in dieser Arbeit bei der Deposition von Ag auf die oben beschriebenen Cu-Schichten beobachtet (Abbildung 4-12). Da die (100)-Oberfläche des $\mathrm{Cu}$ eine vierzählige Symmetrie aufweist, die (111)-Grenzfläche des Ag jedoch dreizählige Symmetrie besitzt, gibt es vier gleichberechtigte Orientierungsmöglichkeiten für die aufwachsende

\footnotetext{
14 Die Annahme einer homogenen Verdichtung ist nur eine grobe Näherung, da die Kräfte, die vom Substrat zur Aufrechterhaltung der Verdichtung aufzubringen sind, nur in der Substratebene wirken. In vertikaler Richtung kann der Film relaxieren. Der angegebene Wert für die Druckspannung ist daher als Mittelwert einer nicht isotropen Druckverteilung zu verstehen.
} 


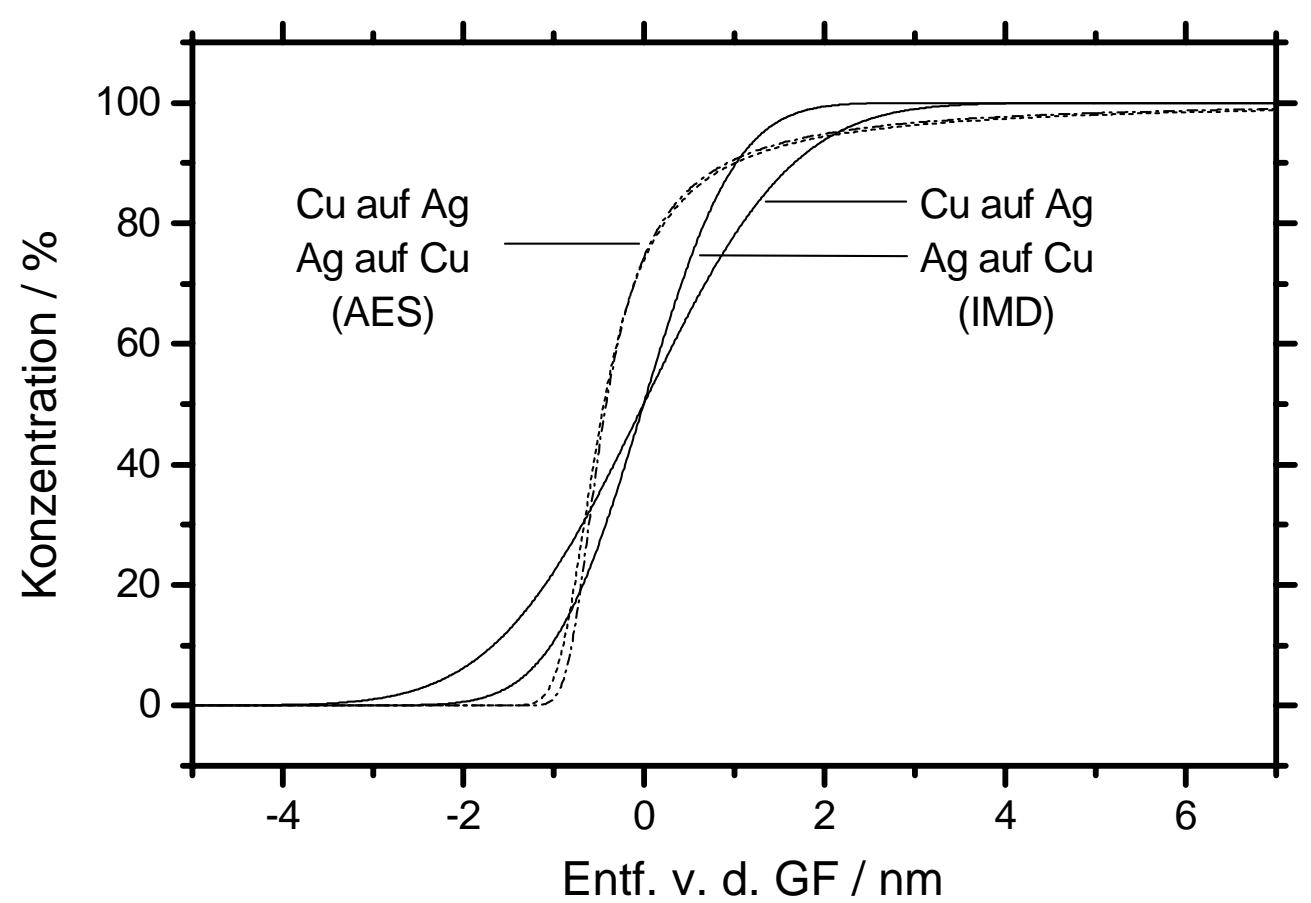

Abbildung 5-1: Vergleich der mittels AES bestimmten mit den in IMD eingesetzten Konzentrationsprofilen

Ag-Schicht (Abbildung 4-14). Innerhalb der Bereiche mit einheitlicher Orientierung wächst die Schicht lokal epitaktisch auf, wie anhand der schmalen in-plane-Textur in Abbildung 4-12 zu erkennen ist.

Die Kleinwinkel-Röntgenstreuung zeigt, daß die ursprüngliche Oberflächenrauhigkeit der $\mathrm{Cu}-\mathrm{Schicht}$ nach der Deposition des $\mathrm{Ag}$ in Form der $\mathrm{Cu}-\mathrm{Ag}$-Grenzfläche praktisch erhalten bleibt. Die Breite der Grenzfläche ist nur unwesentlich um 0,1 bis 0,2 nm erhöht. Das ist weniger als nach dem Implantationsmodell (Kap. 3.3.2) zu erwarten wäre. Möglicherweise sind die mit der Implantation einhergehenden Druckspannungen so hoch (das Ag-Atom ist deutlich größer als das $\mathrm{Cu}$-Atom), daß eine Segregation der implantierten Ag-Atome an die Cu-Oberfläche einsetzt. Auch thermodynamisch wird eine Oberflächensegregation des Ag begünstigt, da die freie Oberflächenenergie von Ag kleiner als die von $\mathrm{Cu}$ ist [100]. Die Mischungsenthalpie des Systems $\mathrm{Cu}-\mathrm{Ag}$ ist mit $4,8 \mathrm{~kJ} / \mathrm{mol}$ dagegen $\mathrm{zu}$ klein, um bei Raumtemperatur als treibende Kraft für eine Entmischung zu wirken [15,53].

Trotz der relativ scharfen Grenzfläche zeigt die Augerelektronenspektroskopie, daß während der Deposition des Ag ein geringer Anteil des Cu über mehrere Nanometer an 
der Oberfläche nachweisbar bleibt (Abbildung 3-14). Die aus der Oberflächenkonzentration bestimmte Konzentration in der Grenzfläche (Abbildung 3-15) zeigt dementsprechend einen stark asymmetrischen Verlauf, mit einem steilen Anstieg der Ag-Konzentration bis etwa $1 \mathrm{~nm}$ und einem daran anschließenden flachen Auslaufen einige Nanometer in die Cu-Schicht hinein. Die Auswertung der Kleinwinkel-Röntgenstreuung dagegen ist beschränkt auf die Simulation mit symmetrischen Konzentrationsprofilen in den Grenzflächen [27]. Abbildung 5-1 zeigt die in der Simulation eingesetzte Gaußsche Fehlerfunktion im Vergleich mit dem aus den AES-Messungen berechneten Konzentrationsprofil. Obwohl die symmetrische Fehlerfunktion den mittels AES bestimmten Konzentrationsverlauf nicht nachbilden kann, ist nach der Deposition von $\mathrm{Ag}$ auf $\mathrm{Cu}$ bzw. $\mathrm{Cu}$ auf $\mathrm{Ag}$ die Grenzflächenbreite in beiden Fällen etwa gleich groß. Wie schon erwähnt, sind etwa $0,6 \mathrm{~nm}$ der Grenzflächenbreite auf die Rauhigkeit der $\mathrm{Cu}$-Schicht zurückzuführen. Der restliche Teil, insbesondere die nur langsam auf 100\% ansteigende Ag-Konzentration, wird durch in der Ag-Schicht gelöstes $\mathrm{Cu}$ hervorgerufen, welches beim Wachstum der AgSchicht „mitgeschleppt“ und zunehmend verdünnt wird.

\subsubsection{Schichtpakete Si-(Cu-Ag)}

Ein Cu-Ag-Schichtpaket auf Si kann nicht einfach als Abfolge der oben beschriebenen $\mathrm{Cu}$-Ag-Doppelschichtstruktur beschrieben werden. Wie Abbildung 4-12 zeigt, wächst das auf eine $\mathrm{Ag}(111)$-Schicht deponierte $\mathrm{Cu}$ ebenfalls in der (111)-Orientierung auf und unterscheidet sich demnach von der untersten $\mathrm{Cu}(100)$-Schicht. Dieser Wachstumsmodus wird auch von Horng et al. beobachtet [101]. Obwohl die Gitterfehlpassung zwischen $\mathrm{Cu}$ und $\mathrm{Ag}$ sehr groß ist, wird epitaktisches Wachstum über viele Doppelschichten beobachtet (Abbildung 4-12), wobei jedoch die Güte der inplane-Textur stark abnimmt.

Aufgrund der höheren Oberflächenenergie des $\mathrm{Cu}$ im Vergleich zu Ag [100] ist mit einem Inselwachstum von $\mathrm{Cu}$ auf $\mathrm{Ag} \mathrm{zu}$ rechnen, was mit zunehmender Zahl von Doppelschichten im Schichtpaket zu einer steigenden Rauhigkeit der Grenzflächen führen sollte. Die Simulation der Kleinwinkelstreuung ergibt jedoch, daß kein starkes Inselwachstum vorliegen kann, da die Rauhigkeiten der Ag-Cu- und der $\mathrm{Cu}-\mathrm{Ag}$ Grenzflächen in den Schichtpaketen gegenüber denen der einfachen Doppelschichten kaum verändert sind. Die in den untersten Lagen gebildeten Grenzflächenrauhigkeiten setzen sich dabei vertikal in den darauf deponierten Schichten fort (sog. korrelierte 


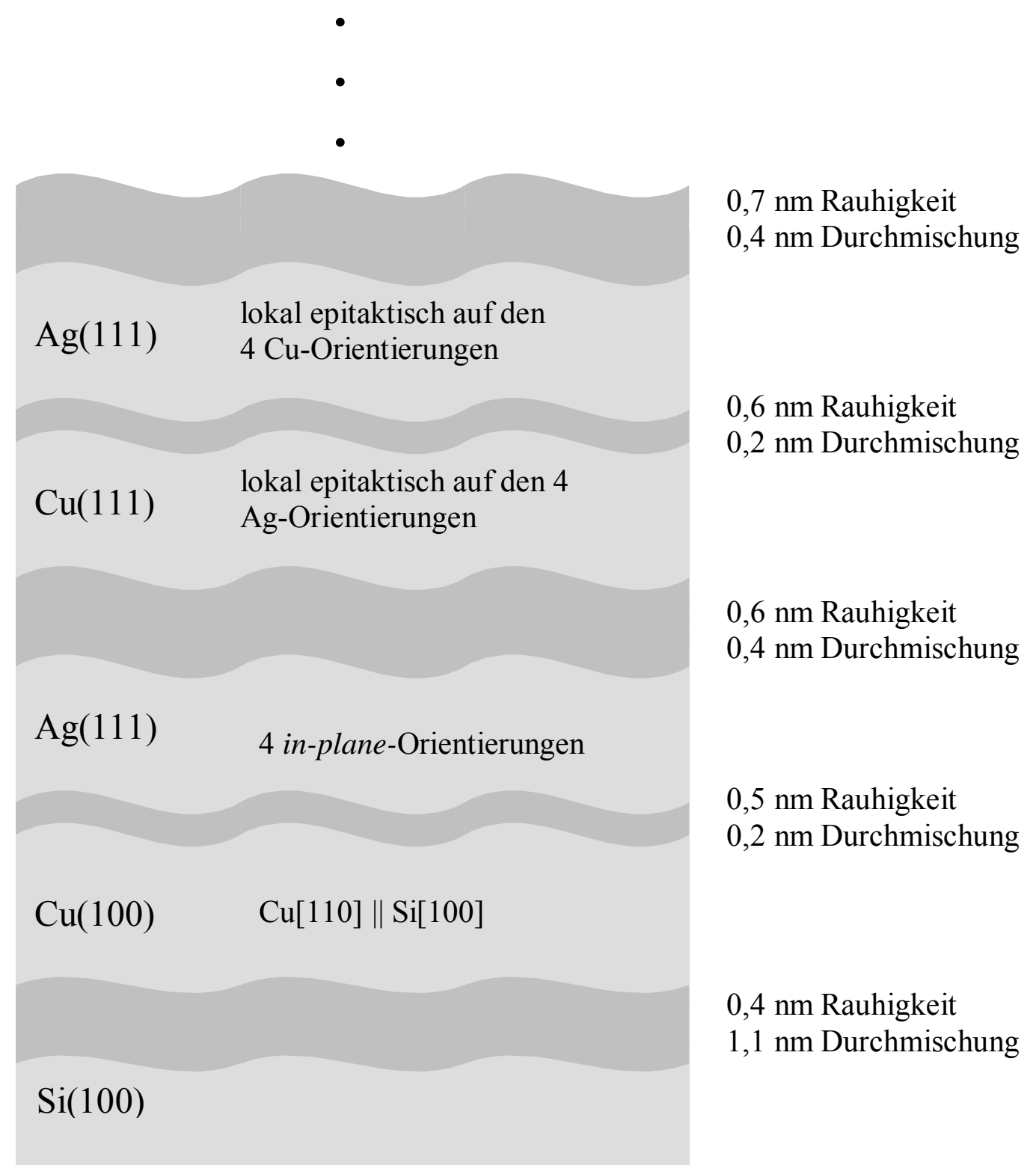

Abbildung 5-2: Schematische Darstellung der Epitaxiebeziehungen und Grenzflächenmorphologien für die Schichtfolge Si-Cu-Ag-... Die Grenzflächenbereiche sind als dunkelgraue Zonen dargestellt, deren Breite die Stärke der Durchmischung verdeutlichen soll.

Rauhigkeiten), wie die diffuse Röntgenstreuung anhand des starken Bragg-Reflexes zeigt (Kap. 4.1.3).

In [101] wird bei Raumtemperatur ebenfalls Lagenwachstum beobachtet, welches nach zwei Monolagen in Inselwachstum umschlägt (Stranski-Krastanov-Wachstum). Erst bei 
höheren Temperaturen wird reines Inselwachstum beobachtet, was darauf hindeutet, daß die Ausbildung von Inseln bei Raumtemperatur kinetisch unterdrückt ist.

Zusammenfassend sind die hier diskutierten Vorstellungen zu Epitaxie und Grenzflächenmorphologie beim Wachstum von $\mathrm{Cu}$-Ag-Schichtpaketen schematisch in Abbildung 5-2 dargestellt. Die angegebenen Rauhigkeiten gelten für eine angenommene jeweilige Einzelschichtdicke von $2 \mathrm{~nm}$ und sind aus den Mittelwerten abgeschätzt, wie sie für ein Schichtpaket aus 20 Doppelschichten mit $84 \mathrm{~nm}$ Gesamtschichtdicke bestimmt wurden.

\subsection{4 $\mathrm{Ag}$ auf $\mathrm{Si}(100)$}

Wie schon für die Deposition von $\mathrm{Cu}$ auf $\mathrm{Si}(100)$ beschrieben, wird auch im Fall von Ag zunächst die amorphe $\mathrm{SiO}_{2}$-Schicht durch die auf das Substrat treffende Ag-Ionen abgesputtert. Auf diese Weise wird die einkristalline Si-Oberfläche freigelegt, was das in diesem System ebenfalls beobachtete epitaktische (100)-Wachstum des Ag erklärt. Im Gegensatz zu Cu wächst das Ag ohne Drehung des Gitters auf der (100)-Oberfläche des Si auf, d. h. Ag[100] || Si[100] (Abbildung 4-11). Die Gitterfehlpassung beträgt in diesem Fall zwar -25\%, kann aber durch die Ausbildung eines 4:3-Koinzidenzgitters auf $0,3 \%$ reduziert werden. Nach [102] hat allerdings die Ag(111)-Grenzfläche mehr Koinzidenzplätze als $\operatorname{Ag}(100)$ mit der Si(100)-Grenzfläche gemeinsam, was ein (111)Wachstum des Ag wahrscheinlich macht. In anderen Arbeiten wird tatsächlich meist ein gemischtes Wachstum aus epitaktischen (100)-Inseln und einem nichtepitaktischen (111)-Anteil beobachtet, sowohl beim Aufdampfen [59,103] als auch bei Sputterdeposition [104] und Aufdampfen mit teilweiser Ionisation und Nachbeschleunigung [105]. Neben (100)- wurde mittels Rastertunnelmikroskopie auch (110)-Wachstum parallel zum (111)-Wachstum nachgewiesen [106].

Diese Beobachtungen zeigen, daß die Wechselwirkung zwischen Si-Substrat und AgFilm zu schwach ist, um die (100)-Orientierung zu favorisieren. Nach [58] ist reines epitaktisches (100)-Wachstum des Ag nur durch eine epitaktische $\mathrm{Cu}$ - und $\mathrm{Pd}$ Zwischenschicht auf dem Si möglich. Um so mehr ist es bemerkenswert, daß die in dieser Arbeit mittel Laserdeposition präparierten Ag-Schichten direkt auf der $\mathrm{Si}(100)$ Oberfläche eine starke (100)-Textur ohne meßbare andere Anteile aufweisen.

Das von Lifshitz et al. vorgeschlagene Subplantationsmodell [13] kann dieses gute heteroepitaktische Wachstum als Folge der hohen kinetischen Energien der 
auftreffenden Teilchen erklären. Nach diesem Modell werden Teilchen mit hinreichend hoher Energie, wie sie z. B. bei der gepulsten Laserdeposition auftreten, einige Monolagen tief unter die Oberfläche des Substrates implantiert („sub-plantiert“) und nehmen Gitterplätze ein, die zuvor von Substratatomen besetzt waren. Durch diesen über mehrere Monolagen ausgedehnten Übergang zwischen Substrat und Film kann die Gitterstruktur des Substrates an den aufwachsenden Film übergeben werden, ohne daß eine starke Wechselwirkung zwischen beiden Elementen vorhanden sein muß. Der große Unterschied in den Gitterkonstanten zwischen Si und Ag muß dabei durch den Einbau von Versetzungen in die Grenzfläche kompensiert werden.

Diese Interpretation wird von den Ergebnissen der Kleinwinkel-Röntgenstreuung gestützt, die eine Breite der Si-Ag-Grenzfläche von 1,2 bis 1,6 nm zeigen. Das ist etwa $1 \mathrm{~nm}$ größer als die ursprüngliche Rauhigkeit des Si-Substrates und somit kann dieser Wert als durchmischter Anteil der Grenzfläche angesehen werden. Si und Ag sind bei Raumtemperatur nicht mischbar, und es wird bei der Deposition mit geringen kinetischen Energien stets eine atomar scharfe Grenzfläche beobachtet [104,107]. Die Durchmischung der Si-Ag-Grenzfläche kann demnach den implantierten Ag-Ionen zugeschrieben werden.

Als Folge der Implantation der Ag-Ionen sind Druckspannungen zu erwarten, die sich als erhöhte Dichte bei der Simulation der Kleinwinkel-Röntgenstreuung bemerkbar machen (Abbildung 4-6). Mit dem Elastizitätsmodul des Ag von 83 GPa läßt sich aus der um maximal 7\% erhöhten Dichte unter der Annahme einer homogenen Verdichtung des Materials (siehe Fußnote 14 auf S. 84) eine maximale Druckspannung von $2 \mathrm{GPa}$ errechnen. Dieser hohe Wert tritt nur bei sehr dünnen Schichten auf und reduziert sich bei Schichtdicken über $20 \mathrm{~nm}$ auf Null, im Gegensatz zu den $\mathrm{Cu}-\mathrm{Schichten}$, bei denen eine (geringe) Druckspannung bis $\mathrm{zu}$ großen Schichtdicken beobachtet wurde. Die direkte Bestimmung der Druckspannung in laserdeponierten bis zu $100 \mathrm{~nm}$ dicken AgFilmen führt zu einem mittleren Wert von $450 \mathrm{MPa}$ [57].

Für die dünne Ag-Schicht unter der Si-Oberfläche, die für die korrekte Beschreibung der Kleinwinkel-Röntgendiffraktogramme erforderlich ist, konnte bisher keine Erklärung gefunden werden.

Die Oberflächenrauhigkeit der wachsenden Ag-Schicht zeigt eine Abhängigkeit von der Schichtdicke, wie sie von Kardar, Parisi und Zhang in einem universellen Modell 
beschrieben wurde [108]. Danach steigt die Rauhigkeit $\sigma$ nach einer Potenz $\beta$ der Schichtdicke $d$ an:

$$
\sigma \sim d^{\beta}
$$

Numerische Simulationen zeigen, daß bei vernachlässigbarer Oberflächendiffusion der Exponent $\beta$ zwischen 0,24 und 0,25 liegt [109]. Bei starker Oberflächendiffusion reduziert sich $\beta$ auf 0,20 [110]. Der hier gemessene Wert von 0,23 liegt zwischen diesen beiden Grenzfällen und ist vergleichbar mit dem Exponenten, den Thompson et al. für auf Si aufgedampfte Ag-Schichten angeben $(\beta=0,26$ [111]). Allerdings ist die absolute Rauhigkeit der hier mittels Laserdeposition präparierten Ag-Schichten deutlich geringer, wie es schon bei den $\mathrm{Cu}-\mathrm{Schichten}$ der Fall war. In [111] wird beispielsweise $\sigma=3 \mathrm{~nm}$ für eine $73 \mathrm{~nm}$ dicke Schicht angegeben, während in dieser Arbeit 1,2 nm bei einer Dicke von 93 nm gemessen wurde.

\subsubsection{Cu auf $\mathrm{Ag}(100)$}

Wird $\mathrm{Cu}$ auf der so präparierten Ag-Oberfläche deponiert, wächst es epitaktisch auf der

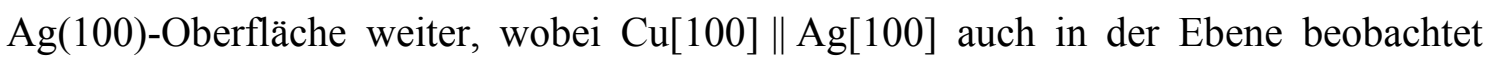
wird (Abbildung 4-11). Dies steht im Gegensatz zum (111)-Wachstum des Ag auf einer $\mathrm{Cu}(100)$-Oberfläche (Kap. 5.1.2) und zum Wachstumsmodus von aufgedampften $\mathrm{Cu}$ Schichten auf $\operatorname{Ag}(100)$ : Aufgrund der starken Gitterfehlpassung von 13\% beim direkten epitaktischen (100)-Wachstum des Cu-fcc-Gitters wird für die ersten Monolagen oft eine metastabile bcc-Struktur des $\mathrm{Cu}$ beobachtet [112-114], die bei einer Fehlpassung von nur 0,3\% eine praktisch perfekte Anpassung der Gitterkonstanten ermöglicht [112].

Dieses bcc-Wachstum kann für maximal einige Nanometer aufrechterhalten werden, danach wird eine Mischung aus (100)- und (110)-fcc-Wachstum beobachtet [115].

Der Grund für das gute epitaktische (100)-Wachstum der auf $\operatorname{Ag}(100)$ laserdeponierten Cu-Schichten kann wie schon im Fall der Deposition von Ag auf Si(100) auf die Implantation der $\mathrm{Cu}$-Ionen unter die Ag-Oberfläche erklärt werden, wo sie AgGitterplätze besetzen und so die fcc-Gitterstruktur des Ag übernehmen. Aufgrund der deutlich höheren Oberflächenenergie des Cu im Vergleich zum Ag [100] und der relativ geringen Grenzflächenenergie zwischen $\mathrm{Cu}$ und $\mathrm{Ag}$ von $0,27 \mathrm{~J} / \mathrm{m}^{2}$ [116] wäre auch eine thermodynamisch getriebene Durchmischung an der Oberfläche denkbar. Dies wird aber nicht beobachtet [117], weshalb dieser Wachstumsmodus bei der Deposition von Teilchen mit thermischen Energien nicht auftritt. 
Die Implantation des $\mathrm{Cu}$ wird nach der Simulation der Kleinwinkel-Röntgenstreuung in Form einer breiteren Ag-Cu-Grenzfläche sichtbar. Im Vergleich mit der Rauhigkeit der ursprünglichen Ag-Oberfläche von 0,9 nm beträgt die Grenzflächenbreite 1,3 nm. Die Differenz von 0,4 nm kann als Ausdehnung des durchmischten Bereiches aufgefaßt werden. Wie schon im Fall der Deposition von Ag auf $\mathrm{Cu}$ kann das als Grundlage für die Simulation der Kleinwinkel-Röntgenstreuung eingesetzte symmetrische Konzentrationsprofil den aus den AES-Messungen bestimmten, asymmetrischen Konzentrationsverlauf nicht korrekt beschreiben (Abbildung 5-1). Trotz dieser Einschränkung ist der durch die beiden Methoden unabhängig bestimmte Bereich, in dem sich der größte Teil des Übergangs zwischen $\mathrm{Ag}$ und $\mathrm{Cu}$ vollzieht, in seiner Ausdehnung vergleichbar. Offensichtlich führt die begrenzte Auflösung der AES, die mit etwa 0,7 nm nur knapp unterhalb der gemessen Grenzflächenbreiten liegt, jedoch dazu, daß die aus der Kleinwinkel-Röntgenstreuung bestimmte und schon früher beobachtete [11] unterschiedliche Breite von $\mathrm{Cu}-\mathrm{Ag}$ - und $\mathrm{Ag}-\mathrm{Cu}-\mathrm{Grenzfläche} \mathrm{mit} \mathrm{dieser}$ Methode nicht auflösbar ist.

\subsubsection{Schichtpakete Si- $(\mathrm{Ag}-\mathrm{Cu})_{\mathrm{n}}$}

Mit dem beschriebenen (100)-Wachstum des $\mathrm{Cu}$ auf der (100)-Ag-Schicht ist dieselbe Situation erreicht, wie sie nach der Deposition von $\mathrm{Cu}$ auf $\mathrm{Si}(100)$ beobachtet wurde (Kap. 5.1.1). Das Wachstum folgender Ag- und $\mathrm{Cu}$-Schichten vollzieht sich demzufolge wie in Kap. 5.1.2 und 5.1.3 beschrieben. Beide Schichtfolgen führen letztendlich zu einem lokal-epitaktischen Wachstum von (111)-Domänen in den $\mathrm{Cu}$ - und $\mathrm{Ag}$ Schichten, wobei sich Rauhigkeiten vertikal korreliert fortsetzen. Der Unterschied liegt nur in der in-plane-Orientierung der Schichten, die im Fall der Schichtfolge Si-Cu... aufgrund der Orientierungsbeziehung zwischen $\mathrm{Cu}$ und $\mathrm{Si}$ eine $45^{\circ}$-Drehung relativ zum Substrat erfährt. Die Güte der in-plane-Textur ist in beiden Fällen etwa gleich groß (vgl. Abbildung 4-11 und 4-12).

Analog zum Wachstum der Schichtfolge Si-Cu-Ag... sind die Ergebnisse für die Schichtfolge Si-Ag-Cu... schematisch in Abbildung 5-3 dargestellt. Wiederum gelten die angegebenen Grenzflächenrauhigkeiten für eine angenommene jeweilige Einzelschichtdicke von $2 \mathrm{~nm}$. 


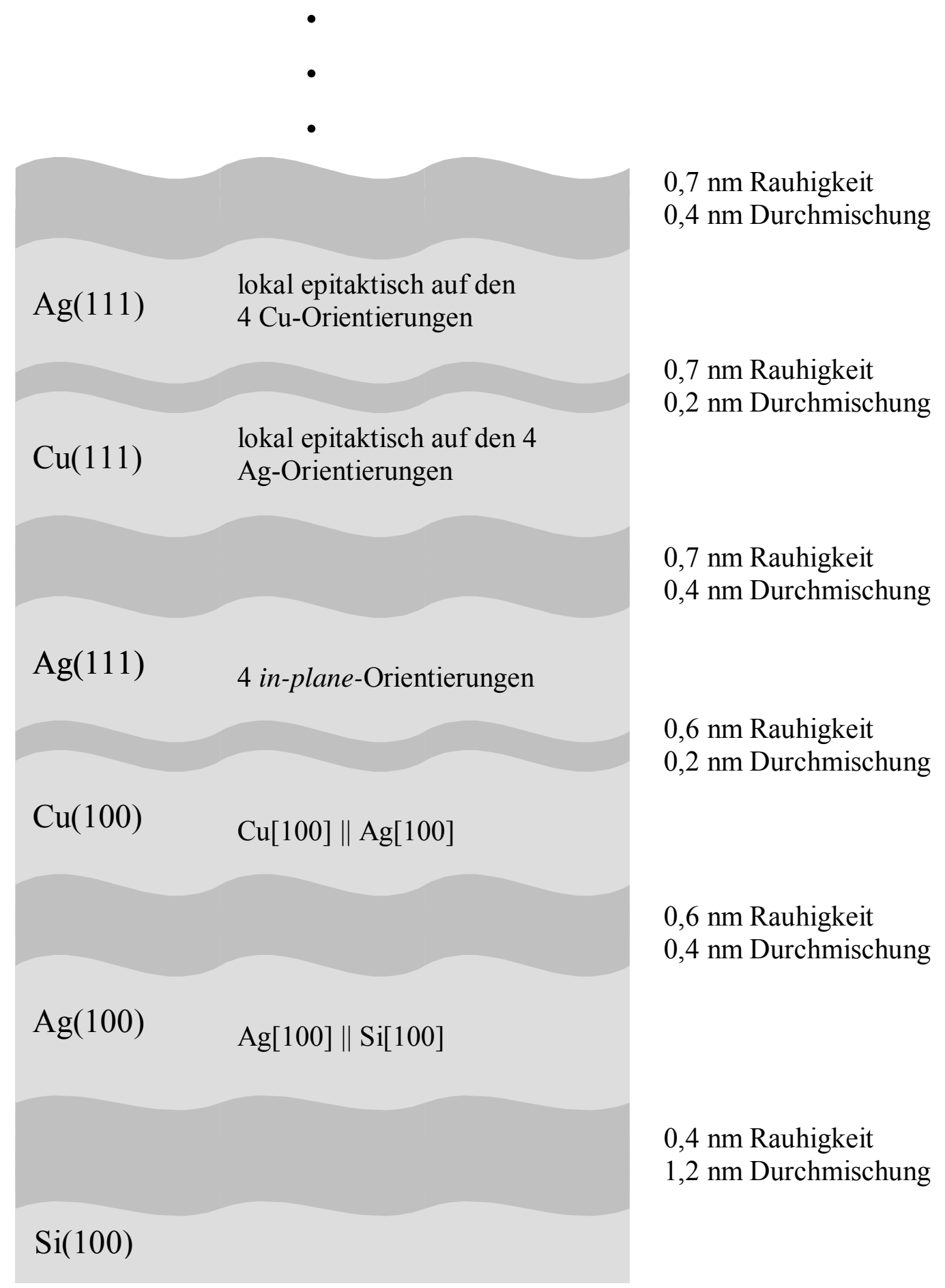

Abbildung 5-3: Schematische Darstellung der Epitaxiebeziehungen und Grenzflächenmorphologien für die Schichtfolge Si-Ag-Cu... Die Grenzflächenbereiche sind als dunkelgraue Zonen dargestellt, deren Breite die Stärke der Durchmischung verdeutlichen soll. 


\subsection{Fe-Ag-Schichtsysteme}

Die Fe-Ag-Schichtsysteme wurden neben der AES hauptsächlich mittels Mößbauerspektroskopie untersucht, da sich das ${ }^{57} \mathrm{Fe}$-Isotop für diese Messungen anbietet und die Kleinwinkel-Röntgenstreuexperimente keine zuverlässigen Informationen über die Grenzfläche lieferten. Schon die Simulation der FeEinzelschichten auf Si war in keine gute Übereinstimmung mit den gemessenen Diffraktogrammen $\mathrm{zu}$ bringen (Abbildung 4-3). Offensichtlich kann die Si-FeGrenzfläche nicht einfach durch einen stetigen Konzentrationsübergang beschrieben werden. Über den Aufbau dieser Grenzfläche nach der Deposition einer Fe-Schicht bei Raumtemperatur existieren widersprüchliche Auffassungen. Diese reichen von einer scharfen Grenzfläche [118] über die Bildung verschiedener Silizide ( $\mathrm{FeSi}_{2}[119,120]$, FeSi [121,122], $\mathrm{Fe}_{3} \mathrm{Si}$ [123]) bis hin zu einer amorphen Si-Fe-Zwischenschicht [124]. Bei der Schichtpräparation mittels gepulster Laserdeposition ist aufgrund der hohen kinetischen Energie der Fe-Ionen, deren Anteil im Laserplasma bei der hier verwendeten Energiedichte am Target mindestens 90\% beträgt [10], mit ihrer Implantation in die Si-Oberfläche und damit mit einer durchmischten Si-Fe-Grenzfläche zu rechnen. Die Kleinwinkel-Röntgenstreuung läßt auf eine Grenzflächenbreite von 1,5 nm schließen, was den Werten für die $\mathrm{Si}-\mathrm{Cu}$ - und die Si-Ag-Grenzfläche gleichkommt und durch eine rein ballistische Durchmischung erklärbar ist. Die thermodynamisch getriebene Bildung einer breiten Silizidphase wurde erst nach dem Aufheizen der Schicht beobachtet [125].

Nach [124] wächst das Fe auf der durchmischten Si-Fe-Grenzfläche nanokristallin auf, wobei in [126] der Wachstumsmodus als Volmer-Weber (Inselwachstum) identifiziert wurde. Wie schon im Fall der Deposition von Ag auf Si (Kap. 5.1.4) sollte auch bei der Laserdeposition von Fe die Erzeugung von Oberflächendefekten durch die energiereichen Fe-Ionen zu einer höheren Inseldichte und damit einer glatteren Schicht führen. Dies kann durch die Ergebnisse der Kleinwinkel-Röntgenstreuung bestätigt werden, die als obere Abschätzung eine Rauhigkeit von $2 \mathrm{~nm}$ für Schichten bis $44 \mathrm{~nm}$ Dicke liefern. 


\subsection{1 $\mathrm{Ag}$ auf $\mathrm{Fe}$}

Bei der Deposition von Ag auf Fe wurde mittels CEMS eine Durchmischung der FeAg-Grenzfläche von 1,5 nm gemessen. Dies entspricht etwa der Grenzflächenbreite, in der nach den Ergebnissen der AES der größte Teil der Konzentrationsänderung vollzogen ist (Abbildung 3-15). Wie schon im Fall der Cu-Ag-Grenzfläche gibt es auch hier einen geringen Anteil Fe, der bis zu einige Nanometer weit in die deponierte AgSchicht hineinreicht. Da das System Fe-Ag stark entmischend und die Oberflächenenergie von $\mathrm{Ag}$ deutlich kleiner als die des Fe ist [127], gibt es keine treibende Kraft für die Diffusion des Ag in die Fe-Schicht. Die Durchmischung muß demnach durch die Implantation der energiereichen Ag-Ionen verursacht sein. Die Implantation von $\mathrm{Ag}$ in die bcc-Kristallstruktur des $\mathrm{Fe}$ führt dazu, daß das im Gleichgewicht in der fcc-Struktur vorliegende Ag in eine metastabile bcc-Struktur gezwungen wird, was aufgrund des geringen Energieunterschiedes zwischen den beiden Kristallstrukturen [128,129] und der nicht $\mathrm{zu}$ großen Gitterfehlpassung von 10\% zwischen bcc-Fe und bcc-Ag [21] möglich wird. Das Wachstum von bcc-Ag und der Übergang zur fcc-Gleichgewichtsstruktur wurde von S. Fähler detailliert untersucht [21] und mit Hilfe der Bain-Transformation [130] theoretisch beschrieben.

Im Gegensatz zum System $\mathrm{Cu}-\mathrm{Ag}$ sind homogene Fe-Ag-Legierungen aufgrund ihrer stark positiven Mischungsenthalpie von $28 \mathrm{~kJ} / \mathrm{mol}$ im mittleren Konzentrationsbereich nicht stabil und entmischen bei Raumtemperatur zu einer heterogenen Legierung. Die Grenzkonzentrationen, die sich bei der Laserdeposition von Fe-Ag-Legierungen einstellen, wurden von M. Störmer zu 16\% Ag im Fe und unter 1\% Fe im Ag bestimmt [16]. Demnach kann sich bei der Deposition von Ag auf Fe keine Grenzfläche mit einem kontinuierlichen Konzentrationsverlauf ausbilden. Nur bei kleinen AgKonzentrationen $\mathrm{zu}$ Beginn der Deposition ist mit einer homogenen Oberflächenlegierung zu rechnen. Erreicht der Ag-Anteil die Grenzkonzentration von 16\%, scheidet sich eine Ag-reiche Phase aus. Aufgrund der kleineren Oberflächenenergie des Ag im Vergleich zu Fe [131] sollte sich diese Phase bevorzugt auf der Oberfläche bilden. Die Ergebnisse der Mößbauerspektroskopie, die einen Anstieg der Konzentration auf maximal 15\% zeigen, passen zu diesem Szenario. 


\subsubsection{Fe auf $\mathrm{Ag}$}

Im umgekehrten Fall der Deposition von Fe auf Ag zeigt eine Betrachtung der Grenzflächen- und Oberflächenenergien, daß eine Diffusion der Ag-Substratatome auf die deponierte Fe-Schicht (bzw. Fe-Inseln) möglich ist. Nach [127] ist $\gamma_{\mathrm{Fe}} \approx 2 \gamma_{\mathrm{Ag}}$ und $\gamma_{\mathrm{Ag}} \approx 2 \gamma_{\mathrm{Ag} / \mathrm{Fe}}$ [132], wobei $\gamma_{\mathrm{Fe}}$ die Oberflächenenergie von $\mathrm{Fe}, \gamma_{\mathrm{Ag}}$ die Oberflächenenergie von $\mathrm{Ag}$ und $\gamma_{\mathrm{Ag} / \mathrm{Fe}}$ die Grenzflächenenergie zwischen $\mathrm{Fe}$ und $\mathrm{Ag}$ bezeichnet. Damit wird die Umwandlung der deponierten Ag-Fe-Struktur in eine AgFe-Ag-Struktur energetisch günstiger:

$$
2 \gamma_{A g / F e}+\gamma_{A g}<\gamma_{A g / F e}+\gamma_{F e}
$$

Tatsächlich wird das „Aufschwimmen“ einer dünnen Ag-Lage beim Aufdampfen von Fe auf Ag bei Raumtemperatur beobachtet [18]. Die AES-Messungen in dieser Arbeit zeigen dagegen, daß bei der Laserdeposition von Fe auf Ag die Ag-Oberfläche schon nach wenig deponiertem Fe vollständig mit Fe bedeckt ist. Dieses unterschiedliche Verhalten kann damit erklärt werden, daß die auf die Fe-Schicht diffundierenden AgAtome durch die auf die Oberfläche treffenden energiereichen Fe-Ionen wieder abgetragen werden. Die Fe-Schicht selbst bleibt von Abtrag weitgehend verschont, da der Eigensputteryield von Fe bei den auftretenden Energien um $100 \mathrm{eV}$ deutlich kleiner ist als der Sputteryield von Ag durch Fe [22]. Dieser als präferentielles Sputtern bei der Sputterdeposition von Legierungen [133] bekannte Effekt ist so groß, daß bei der Präparation von Fe-Ag-Schichtpaketen mittels PLD die Ag-Schichten jeweils zwei bis drei Monolagen dünner sind als nach der Depositionsrate von Ag auf Ag zu erwarten wäre [9]. Damit ist das präferentielle Sputtern stark genug, um den in [11] beschriebenen Abfall des elektrischen Leitwertes bei der Laserdeposition von Fe auf Ag $\mathrm{zu}$ verursachen. Dieser wurde dort hauptsächlich durch die Bildung einer schlecht leitfähigen Legierung durch die Implantation von Fe in die Ag-Schicht erklärt.

Der Wiederabtrag schon deponierten Materials tritt auch bei $\mathrm{Cu}-\mathrm{Ag}$-Schichten auf (siehe Kap. 3.1), führt aber in diesem System zu keinen so deutlichen Effekten, da sich die Sputteryields von $\mathrm{Cu}$ und $\mathrm{Ag}$ nicht so stark unterscheiden.

Neben dem Sputtern der Ag-Oberfläche ist auch mit einer Implantation der Fe-Ionen in die obersten Monolagen der Ag-Schicht zu rechnen. Nach [11] sollten diese bei einer Auftreffenergie von $100 \mathrm{eV}$ im Mittel 0,8 nm tief in die Ag-Oberfläche eindringen, was vergleichbar ist mit der Implantationstiefe von Ag in Fe. Allerdings zeigen sowohl 
Mößbauerspektroskopie als auch Augerelektronenspektroskopie (im Rahmen der Auflösung von $0,7 \mathrm{~nm}$, siehe Kap. 3.3.1) keine bzw. nur eine sehr geringe Durchmischung an der Ag-Fe-Grenzfläche, vergleichbar mit aufgedampften Ag-FeSchichten [134]. Die implantierten Fe-Atome müssen daher schon während der Deposition wieder an die Ag-Oberfläche diffundieren. Die deutlich höhere Oberflächenenergie des Fe läßt dort Inselwachstum vermuten, wie es beim Aufdampfen von Fe auf Ag auch beobachtet wird [134].

Das Herausdiffundieren der Fe-Atome führt dazu, daß der in Kap. 5.2.1 beschriebene subsurface-Wachstumsmodus (unter der Oberfläche) von Ag in Fe in diesem Fall nicht beobachtet wird. Aus diesem Grund, und wegen der deutlich höheren Energie der Fefcc-Kristallstruktur im Vergleich zur bcc-Gleichgewichtsstruktur $(50-60 \mathrm{meV}$ pro Atom [135]), ist nicht mit einer metastabilen Fe-fcc-Phase zu Beginn der Fe-Deposition $\mathrm{zu}$ rechnen. S. Fähler konnte mittels in-situ-RHEED-Messungen während der Laserdeposition von $\mathrm{Fe}$ auf $\mathrm{Ag}$ zeigen, daß das $\mathrm{Fe}$ ohne Übergang in der (zunächst durch Scherung verzerrten) bcc-Struktur aufwächst [21].

Die Kinetik des Entmischungsvorganges bei der Deposition von Fe-AgSchichtsystemen kann durch eine Erhöhung der Depositionsrate reduziert werden. Dies wurde durch die Experimente zur Variation des Target-Substrat-Abstandes gezeigt, die Kap. 4.2.3 beschrieben und diskutiert sind. 


\section{Zusammenfassung}

Ziel dieser Arbeit war es, die Durchmischung und Rauhigkeit der Grenzflächen in laserdeponierten $\mathrm{Cu}$-Ag- und Fe-Ag-Schichtsystemen mittels verschiedenen, sich ergänzenden Methoden quantitativ zu untersuchen. Dazu kamen in-situ Augerelektronenspektroskopie und Ratenmessungen sowie ex-situ durchgeführte Röntgendiffraktometrie- sowie Mößbauerspektroskopiemessungen zum Einsatz.

Die im Vergleich der Messungen mit theoretischen Modellen und Simulationsrechnungen gewonnenen Ergebnisse zeigten insbesondere den starken Einfluß der schnellen Ionen, die bei der gepulsten Laserdeposition auftreten. Diese haben unter den eingesetzten Depositionsbedingungen mit Laserenergiedichten von 3 bis $5 \mathrm{~J} / \mathrm{cm}^{2}$ einen sehr hohen Anteil von mindestens $90 \%$ an der Gesamtzahl der deponierten Teilchen. Sie treffen mit einer breiten Verteilung von Energien (im Mittel etwa $100 \mathrm{eV}$ ) auf dem Substrat bzw. der wachsenden Schicht auf und führen dort zu Absputterprozessen, was sich in den Ratenmessungen am direktesten zeigt. Außerdem werden sie einige Monolagen tief implantiert. Die Simulation des Wachstums laserdeponierter Schichten unter Berücksichtigung der Implantation dieser schnellen Ionen zeigt, daß der Konzentrationsverlauf über die Grenzflächen stark asymmetrisch ist: Auf der dem Substrat zugewandten Seite der Grenzfläche ist ein steiler Anstieg der Konzentration zu verzeichnen (80\% innerhalb von $1 \mathrm{~nm}$ ), während auf der dem Substrat abgewandten Seite die restliche Konzentrationsänderung von $20 \%$ über einen Bereich von mehreren Nanometern vollzogen wird. Dies wurde durch den Vergleich der simulierten Abhängigkeit der Oberflächenkonzentration von der deponierten Schichtdicke mit dem gemessenen Verlauf gezeigt. Nur im Fall der Deposition von Fe auf Ag ergibt die Messung einen steileren Verlauf als nach der Simulation zu erwarten wäre. Im Rahmen der Auflösung der Augerelektronenspektroskopie (0,7 nm bei den hier gemessenen Augerlinien) kann diese Grenzfläche als scharf angesehen werden. Das selbe Ergebnis zeigte auch die Mößbauerspektroskopie an Fe-Ag-Schichtpaketen mit ${ }^{57} \mathrm{Fe}-$ Markerschichten an verschiedenen Stellen der Fe-Einzelschichten (maximale Durchmischung 0,5 nm - vorgegeben durch die Dicke der Markerschicht). Die schärfere Ag-Fe-Grenzfläche wurde durch das starke Sputtern der Ag-Atome durch die Fe-Ionen und eine thermodynamisch getriebene Entmischung der Grenzfläche aufgrund der hohen Mischungsenthalpie des Fe-Ag-Systems von 28 kJ/mol begründet. Es konnte 
gezeigt werden, daß sich durch eine Erhöhung der Depositionsrate über die Verringerung des Target-Substrat-Abstandes diese Entmischung teilweise unterdrücken läßt.

Aus der Simulation der Kleinwinkel-Röntgenreflektometrie, die im Fall der $\mathrm{Cu}-\mathrm{Ag}$ Schichtsysteme eine sehr gute Übereinstimmung mit den Messungen zeigte, konnte die Breite der Grenzflächen in diesem System bestimmt werden. Je nach deponiertem Element ergaben sich dabei unterschiedliche Werte für die Rauhigkeit bzw. Durchmischung $\sigma$. Beispielsweise wurde bei der Deposition von $\mathrm{Cu}$ auf eine $50 \mathrm{~nm}$ dicke Ag-Schicht (Ag-Cu-Grenzfläche) $\sigma=1,3 \mathrm{~nm}$ gemessen, während nach der Deposition von $\mathrm{Ag}$ auf eine $50 \mathrm{~nm}$ dicke $\mathrm{Cu}-\mathrm{Schicht} \sigma \mathrm{zu} 0,8 \mathrm{~nm}$ bestimmt wurde. Durch einen Vergleich mit der Oberflächenrauhigkeit von $\mathrm{Cu}$ - und Ag-Schichten gleicher Dicke konnte der Beitrag von Rauhigkeit und Durchmischung auf $\sigma$ getrennt werden. Demnach ist die Durchmischung bei der Deposition von $\mathrm{Cu}$ auf $\mathrm{Ag}$ mit 0,4 nm deutlich größer als im umgekehrten Fall mit nur 0,1 nm, was frühere Messungen zur Leitfähigkeit laserdeponierter $\mathrm{Cu}-\mathrm{Ag}$-Schichten bestätigt.

Die Oberflächenrauhigkeit selbst ist geringer als bei Raumtemperatur aufgedampften $\mathrm{Cu}$ - und Ag-Filmen. Beispielsweise wurde in dieser Arbeit für eine $93 \mathrm{~nm}$ dicke AgSchicht $\sigma=1,2 \mathrm{~nm}$ gemessen, wohingegen eine aufgedampfte $73 \mathrm{~nm}$ dicke Schicht eine Rauhigkeit von $\sigma=3 \mathrm{~nm}$ aufweist. Dies wurde durch die Erzeugung einer hohen Zahl von Oberflächendefekten durch die bei der Laserdeposition auf die wachsende Schicht auftreffenden schnellen Ionen erklärt, welche als Keimzentren für die auf der Oberfläche diffundierenden Atome dienen und so eine hohe Dichte kleiner Inseln und eine entsprechend geringe Rauhigkeit zur Folge haben.

Auch die Epitaxie laserdeponierter Schichten wird durch die kinetische Energie der Teilchen positiv beeinflußt. So konnte mittels Röntgendiffraktometrie gezeigt werden, daß Ag bei Raumtemperatur auf der Si(100)-Oberfläche epitaktisch in (100)Orientierung mit einer guten in-plane-Textur von $4,1^{\circ}$ und ohne meßbare Anteile anderer Orientierungen aufwächst, was mit konventionellen Depositionsmethoden nur schwer zu erreichen ist. Auch die Epitaxiebeziehungen zwischen $\mathrm{Cu}$ und $\mathrm{Si}(100)$ sowie zwischen den Einzelschichten in Cu-Ag-Schichtsystemen wurden mit dieser Methode bestimmt. Als Erklärung für den guten Epitaxieübertrag wurde in einigen Fällen (Ag auf $\mathrm{Si}, \mathrm{Cu}$ auf $\mathrm{Ag}$ ) das Subplantationsmodell von Lifshitz herangezogen, welches im Gegensatz zu den traditionellen Wachstumsmoden auf der Substratoberfläche (Frank- 
van-der-Merve, Volmer-Weber und Stranski-Krastanov) ein Wachstum der Schicht wenige Monolagen unterhalb der Oberfläche durch die implantierten Ionen beschreibt. Auf diese Weise kann die Kristallstruktur des Substratmaterials über einen Bereich von mehreren Monolagen an die aufwachsende Schicht übertragen werden.

Mit Hilfe der Kleinwinkel-Röntgenreflektometrie konnte für $\mathrm{Cu}$ - und $\mathrm{Ag}$ Einzelschichten die durch die Implantation der Teilchen zu erwartende Verdichtung der Schicht nachgewiesen werden. Aus der im Mittel um 0,3\% erhöhten Dichte der $\mathrm{Cu}$ Schichten (bis zu 7\% für Ag) errechnen sich Druckspannungen von $130 \mathrm{MPa}$ bzw. maximal $2 \mathrm{GPa}$ für $\mathrm{Ag}$, die mit direkten Messungen der Filmspannung bei der Deposition mit energetischen Ionen vergleichbar sind.

Insgesamt konnte in dieser Arbeit quantitativ gezeigt werden, wie die Grenzflächen in laserdeponierten $\mathrm{Cu}-\mathrm{Ag}$ - und $\mathrm{Fe}-\mathrm{Ag}$-Schichten durch die Implantation der schnellen Ionen durchmischt werden, wobei in beiden Systemen eine starke Asymmetrie sowohl in der Stärke der Durchmischung als auch im Konzentrationsprofil der Grenzflächen beobachtet wurde. In $\mathrm{Cu}-\mathrm{Ag}$ konnte der Anteil der Grenzflächenrauhigkeit an der Ausdehnung der Grenzflächen bestimmt werden.

Zusätzlich wurde gezeigt, daß die laserdeponierten $\mathrm{Cu}-\mathrm{Ag}$-Schichten ein hohe Dichte besitzen und mit geringer Oberflächenrauhigkeit gut epitaktisch auf $\operatorname{Si}(100)$ und untereinander aufwachsen. Diese auf die Deposition mit energetischen Teilchen zurückgeführten Eigenschaften der Schichten sind wichtige Voraussetzungen für die Metallisierung von Silizium-Wafern in der Halbleiterindustrie, wo aus Kostengründen und wegen ihrer hohen Depositionsrate hauptsächlich die Sputterdeposition als Beschichtungsverfahren eingesetzt wird. Die Vorteile der hohen kinetischen Energie der Ionen bei der Laserdeposition, welche für den großtechnischen Einsatz zu aufwendig ist, können möglicherweise auch für die industrielle Anwendung durch eine Nachbeschleunigung der zu deponierenden Teilchen nutzbar gemacht werden. Eine solche Methode ist unter dem Namen FCVA (ㅌiltered Cathodic Vacuum $\underline{\text { Arc }}$ ) bekannt und wird seit einiger Zeit auf ihre Tauglichkeit für die Herstellung qualitativ hochwertiger ${ }^{15}$ Beschichtungen auf Silizium untersucht [136,137].

\footnotetext{
${ }^{15}$ Ein wichtiges Kriterium neben den schon genannten ist die maximale Tiefe relativ zur Breite von Leiterbahngräben, die bei der Beschichtung mit Material (Al, $\mathrm{Cu}, \mathrm{Ag}$ ) aufgefüllt werden kann (das sog. aspect ratio). Auch hier zeigt die Deposition mit Teilchen hoher kinetischer Energie bessere Ergebnisse $[138,139]$.
} 


\section{Literatur}

1. S. M. Gorbatkin, D. B. Poker, R. L. Rhoades, C. Doughty, L. A. Berry: $C u$ metallization using a permanent magnet electron cyclotron resonance microwave plasma/sputtering hybrid system; J. Vac. Sci. Technol. B 14 (1996) 1853

2. M. Hauder, J. Gstöttner, W. Hansch, D. Schmitt-Landsiedel: Scaling properties and electromigration resistance of sputtered Ag metallization lines; Appl. Phys. Lett. 78 (2001) 838

3. C.-K. Hu, D. C. Edelstein, C. Uzoh: Comparison of electromigration in submicron $\mathrm{Al}(\mathrm{Cu})$ and $\mathrm{Cu}$ thin film lines; AIP Conf. Proc. 373 (1996) 153

4. M. Setton, J. Van der Spiegel, B. Rothman: Copper silicide formation by rapid thermal processing and induced room-temperature Si oxide growth; Appl. Phys. Lett. 57 (1990) 357

5. H. Mu, Y. Yu, E. Z. Luo et al: Ti, TiN, and Ti-TiN thin films prepared by ion beam assisted deposition as diffusion barriers between $\mathrm{Cu}$ and Si; J. Vac. Sci. Technol. 18 (2000) 2312

6. M. B. Takeyama, A. Noya, and K. Sakanishi: Diffusion barrier properties of ZrN films in the Cu/Si contact systems; J. Vac. Sci. Technol. B 18 (2000) 1333

7. D. B. Chrisey, G. K. Hubler (editors): Pulsed Laser Deposition of Thin Films; Wiley Interscience Publication, 1994

8. H.-U. Krebs: Characteristic Properties of Laser-Deposited Metallic Systems; Int. J. Non-Equil. Proc. 10 (1997) 3

9. K. Sturm, H.-U. Krebs: Quantification of resputtering during pulsed laser deposition; J. Appl. Phys. 90 (2001) 1061

10. S. Fähler, K. Sturm, H.-U. Krebs: Resputtering during the growth of pulsed laser deposited metallic films in vacuum and an ambient gas; Appl. Phys. Lett. 75 (1999) 3766

11. M. Weisheit: Untersuchungen zum Wachstum laserdeponierter metallischer Schichten mittels in-situ Widerstandsmessungen; Diplomarbeit, Universität Göttingen, 1998 
12. S. Fähler, M. Weisheit, K. Sturm, H.-U. Krebs: The interface of a laser deposited $\mathrm{Cu} / \mathrm{Ag}$ multilayers: evidence of the 'subsurface growth mode' during pulsed laser deposition; Appl. Surf. Sci. 154 (2000) 419

13. Y. Lifshitz, S. R. Kasi, J. W. Rabalais: Subplantation model for film growth from hyperthermal species; Phys. Rev. B 41 (1990) 10468

14. K.-H. Hellwege, O. Mandelung (Ed.): Landolt-Börnstein, Neue Serie; IV 2 (1976) S. 250

15. I. M. Reda, P. Schattschneider, K. Riedl, A. Wagendristel, H. Bangert, F. Gautier: Electrical resistivity and electron energy loss spectra of amorphous and crystalline thin Cu-Ag films; Thin Solid Films 116 (1984) 269

16. M. Störmer: Struktur, Mikrostruktur und innere Spannungen laserdeponierter metallischer Legierungen; Dissertation, Universität Göttingen, 1998

17. S. Fähler, M. Weisheit, S. Kahl, K. Sturm, H.-U. Krebs: The interface of laserdeposited Fe/Ag multilayers: evidence for the "subsurface growth mode" during pulsed-laser deposition and examination of the bcc-fcc transformation; Appl. Phys. A 69 (1999) 459

18. D. E. Bürgler, C. M. Schmidt, D. M. Schaller, F. Meisinger, R. Hofer, H.-J. Güntherodt: Optimized epitaxial growth of Fe on Ag(001); Phys. Rev. B 56 (1997) 4149

19. R. J. Hicken, C. Daboo, M. Gester, A. J. R. Ives, S. J. Gray, J. A. C. Bland: Interlayer exchange coupling in epitaxial Fe/Cr/Fe/Ag/GaAs(100) structures; J. Appl. Phys. 78 (1995) 6670

20. Ch. Roth, F. U. Hillebrecht, H. Rose, and E. Kisker: Linear magnetic dichroism in angular resolved Fe 3p core level photoemission; Phys. Rev. Lett. 70 (1993) 3479

21. S. Fähler: Der Einflu $\beta$ der kinetischen Energie der Ionen auf das Wachstum laserdeponierter metallischer Schichten und Schichtpakete: Untersuchungen mit Flugzeitmessungen und RHEED/THEED; Dissertation, Universität Göttingen, 1998

22. K. Sturm: Einfluß einer Argon-Atmosphäre auf die gepulste Laserdeposition von Metallschichten; Dissertation, Universität Göttingen, 2000

23. D. Briggs, M. P. Seah: Practical Surface Analysis; Vol.1: Auger and x-ray photoelectron spectroscopy; $2^{\text {nd }}$ ed. Wiley 1990

24. H. Lüth: Surfaces and Interfaces of Solid Materials; $3^{\text {rd }}$ ed. Springer 1997, S. 156 
25. B. D. Cullity: Elements of X-Ray Diffraction; Addison-Wesley Publishing Company Inc., Massachusets, 1987

26. C. Kittel: Introduction to Solid State Physics; John Wiley \& Son, $6^{\text {th }}$ ed. (1986)

27. D. L. Windt: IMD - Software for modeling the optical properties of multilayer films; Computers in Physics, 12 (1998) 360

28. V. Holy, U. Pietsch, T. Baumbach: High-resolution X-ray scattering from thin films and multilayers; Springer, 1999

29. D. G. Stearns: The scattering of x-rays from nonideal multilayer structures; J. Appl. Phys. 65 (1989) 491)

30. M. Tolan: X-ray scattering from soft-matter thin films: materials and basic research; Springer, 1999

31. D. Barb: Grundlagen und Anwendungen der Mößbauerspektroskopie; AkademieVerlag Berlin, 1980

32. U. Gonser (editor): Mössbauer spectroscopy II; Topics in current physics, vol. 25, Springer-Verlag Berlin-Heidelberg-New York, 1978

33. G. U. Nienhaus: Mößbauerspektroskopie; Script zur Vorlesung, Uni Ulm, 2001

34. P. Schaaf: Laser nitriding of metals; Progress in Mat. Sci. 47 (2002) 1

35. P. Schaaf, A. Krämer, F. Aubertin, U. Gonser: Phase, depth and orientation analysis by simultaneous conversion electron, conversion X-ray and transmission Mössbauer spectroscopy; Z. Metallkunde 82 (1991) 815

36. P. Schaaf, A. Krämer, L. Blaes, G. Wagner, F. Aubertin, U. Gonser: Simultaneous conversion electron, conversion X-ray and transmission Mössbauer spectroscopy; Nucl. Instr. Methods B 53 (1991) 184

37. S. Delage, B. Legrand, F. Soisson: Dissolution modes of $\mathrm{Fe} / \mathrm{Cu}$ and $\mathrm{Cu} / \mathrm{Fe}$ deposits; Phys. Rev. B 58 (1998) 15810

38. Th. Detzel, N. Memmel: Substrate diffusion in metastable ultrathin films: Iron on Cu(001); Phys. Rev. B 49 (1994) 5599

39. M. Störmer, K. Sturm, S. Fähler, M. Weisheit, J. Winkler, S. Kahl, P. Kesten, A. Pundt, M. Seibt, S. Senz, H.-U. Krebs: Study of laser deposited metallic thin films by a combination of high-resolution ex-situ and time-resolved in-situ experiments; Appl. Phys. A 69 (1999) 455 
40. S. Fähler, M. Weisheit, H.-U. Krebs: In-situ characterization of laser deposited Fe/Ag multilayers by a combination of time-of-flight, RHEED and resistance measurements; Mat. Res. Soc. Symp. Proc. 502 (1998) 139

41. J. Noetzel, K. Brand, H. Geisler, A. Gorbunov, A. Tselev, E. Wiesner, W. Möller: Structural Investigations of laser-deposited Fe/Al multilayers; Appl. Phys. A 68 (1999) 497

42. K. Meyer, I. K. Schuller, C. M. Falco: Thermalization of sputtered atoms; J. Appl. Phys. 52 (1981) 5803

43. W. E. King, R. E. Benedek: Computer simulation study of the displacement threshold-energy surface in Cu; Phys. Rev. B 23 (1981) 6335

44. G. Betz, K. Wien: Energy and angular distributions of sputtered particles; Int. J. Mass Spect. 140 (1994) 1

45. J. F. Ziegler (editor): The Stopping and Ranges of Ions in Matter; Pergamon Press, 1980

46. F. Garrelie, J. Aubreton, A. Catherinot: Monte Carlo simulation of the laser-induced plasma plume expansion under vacuum: Comparison with experiment; J. Appl. Phys. 83 (1998) 5075

47. J. C. S. Kools, S. H. Brongersma, E. van de Riet, J. Dieleman: Concentrations and velocity distributions of positive ions in laser ablation of copper; Appl. Phys. B 53 (1991) 125

48. P. E. Dyer: Electrical characterization of plasma generation in $\mathrm{KrF}$ laser $\mathrm{Cu}$ ablation; Appl. Phys. Lett. 55 (1989) 1630

49. S. Metev, M. Ozegowski, G. Sepold, S. Burmester: Plasma parameters in pulsed laser-plasma deposition of thin films; Appl. Surf. Sci. 96 (1996) 122

50. R. J. von Gutfeld, R. W. Dreyfus: Electronic probe measurements of pulsed copper ablation at 248 nm; Appl. Phys. Lett. 54 (1989) 1212

51. R. A. Bingham, P. L. Salter: Materials analysis by laser-probe mass spectrometry; Int. J. Mass Spectr. Ion. Phys. 21 (1976) 133

52. M. Störmer: Strukturuntersuchungen an laserdeponierten metallischen Legierungen; Diplomarbeit, Universität Göttingen, 1995

53. K. L. Chopra, A. P. Thakoor, S. K. Barthwal, P. Nath: Electron Transport Properties of Amorphous Cu-Ag Films; Phys. Stat. Sol. (a) 40 (1977) 247 
54. S. K. Ghandi: VLSI fabrication principles: Silicon and Gallium Arsenide; Wiley, New York, 1983, S. 373

55. J. A. Thornton: Influence of apparatus geometry and deposition conditions on the structure and topography of thick sputtered coatings; J. Vac. Soc. Technol. 11 (1974) 666

56. E. Süske: Laserdeposition von Polymer-Metall-Schichten; Diplomarbeit, Universität Göttingen, 2001

57. T. Scharf: Mechanische Spannungen und Struktur laserdeponierter metallischer Filme in Abhängigkeit von der Teilchenenergie; Diplomarbeit, Universität Göttingen, 2001

58. C. Chang: Deposition of (100) Au, Ag, Pd, Pt, and Fe on (100) Si using different metal seed layers; J. Vac. Sci. Technol. A 9 (1991) 98

59. R. Naik, C. Kota, B. U. M. Rao, G. W. Auner: Study of epitaxial growth of Ag on hydrogen terminated Si(111) and Si(100) surfaces; J. Vac. Sci. Technol. A 12 (1994) 1832

60. A. Zur, T. C. McGill: Lattice match: An application to heteroepitaxy; J. Appl. Phys. 55 (1984) 378

61. A. Crespo-Sosa; P. Schaaf; W. Bolse; K.-P. Lieb; M. Gimbel; U. Geyer; C. Tosello: Irradiation effects in Ag-Fe bilayers: ion-beam mixing, recrystallization, and surface roughening; Phys. Rev. B 53 (1996) 14795

62. M. Neubauer; K.-P. Lieb; P. Schaaf; M. Uhrmacher: Ion-beam mixing of Ag/Fe and In/Fe layers studied by hyperfine techniques; Phys. Rev. B 53 (1996) 10237

63. H.-U. Krebs, Y. Luo, M. Störmer, A. Crespo-Sosa, P. Schaaf, W. Bolse: Structural changes during Ar-ion irradiation of laser-deposited Fe/Ag multilayers; Appl. Phys. A 61 (1995) 591

64. F. Landry, P. Schaaf: WinISO fit program for Mössbauer spectra; unpublished

65. H. D. Jakubke, H. Jeschkeit: Brockhaus Chemie; 5. Aufl., VEB F. A. Brockhaus Verlag Leipzig, 1987

66. P. Schaaf, M. Weisheit, H.-U. Krebs: Materials Surface Processing spied by Hyperfine Interactions; Acta Physica Polonica A 100 (2001) 699

67. P. Ballone, S. Rubini: An embedded atom study of an amorphous metal surface: $P d_{80} S i_{20}$; Surf. Sci. 342 (1995) L1116 
68. B. Böddeker, H. Teichler: Dynamics near free surfaces of molecular dynamics simulated $N i_{0.5} Z r_{0.5}$ metallic glass films; Phys. Rev. E 59 (1999) 1948

69. F. Seitz, J. S. Koehler: Displacement of atoms during irradiation; Solid State Phys. 2 (1956) 305

70. E. M. Bringa, R. E. Johnson: Coulomb Explosion and Thermal Spikes; Phys. Rev. Lett. 88 (2002) 165501

71. B. W. Karr, L. D. Madsen, J.-E. Sundgren: Morphology and microstructure of epitaxial $\mathrm{Cu}(001)$ films grown by primary ion deposition on Si and Ge substrates; J. Appl. Phys. 80 (1996) 6699

72. C. A. Davis: A simple model for the formation of compressive stress in thin films by ion bombardment; Thin Solid Films 226 (1993) 30

73. T. J. Colla, H. M. Urbassek, K. Nordlund, R. S. Averback: Ion induced mixing and demixing in the immiscible Ni-Ag system; Phys. Rev. B 63 (2001) 104206

74. H.-U. Krebs, M. Hamp, S. Fähler, M. Störmer, K. Sturm, M. Weisheit: Formation of metallic systems far from equilibrium by pulsed laser deposition; Mat. Res. Soc. Symp. Proc. 481 (1998) 575

75. P. Bai, G.-R. Yang, T.-M. Lu: Study of interface impurity sputtering in partially ionized beam deposition of Cu on Si; J. Appl. Phys. 68 (1990) 3619

76. V. A. Burrows, Y. J. Chabal, G. S. Higashi, K. Raghavachari, S. B. Christman: Infrared spectroscopy of Si(111) surfaces after HF treatment: Hydrogen termination and surface morphology; Appl. Phys. Lett 53 (1988) 998

77. N. Hirashita, M. Kinoshita, I. Aikawa, T. Ajioka: Effects of surface hydrogen on the air oxidation at room temperature of HF-treated Si(100) surfaces; Appl. Phys. Lett. 56 (1990) 451

78. H. Jiang, T. J. Klemmer, J. A. Barnard, E. A. Payzant: Epitaxial growth of Cu on Si by magnetron sputtering; J. Vac. Sci. Technol. A 16 (1998) 3376

79. N. Razek, K. Otte, T. Chassé, D. Hirsch, A. Schindler, F. Frost, B. Rauschenbach: GaAs surface cleaning by low energy hydrogen ion beam treatment; J. Vac. Sci. Technol. A 20 (2002) 1492

80. C.-A. Chang, J. C. Liu, J. Angilello: Epitaxy of (100) Cu on (100) Si by evaporation near room temperatures: In-plane epitaxial relation and channeling analysis; Appl. Phys. Lett. 57 (1990) 2239 
81. E. T. Krastev, L. D. Voice, R. G. Tobin: Surface morphology and electric conductivity of epitaxial Cu(100) films grown on H-terminated Si(100); J. Appl. Phys. 79 (1996) 6865

82. B. G. Demczyk, R. Naik, G. Auner, C. Kota, U. Rao: Growth of $C u$ films on hydrogen terminated Si(100) and Si(111) surfaces; J. Appl. Phys. 75 (1994) 1956

83. I. Hashim, B. Park, H. A. Atwater: Epitaxial growth of $\mathrm{Cu}(001)$ on $\mathrm{Si}(001)$ : Mechanisms of orientation development and defect morphology; Appl. Phys. Lett. 63 (1993) 2833

84. F. J. Walker, E. D. Specht, R. A. McKee: Film/substrate registry as measured by anomalous $x$-ray scattering at a reacted, epitaxial Cu/Si(111) interface; Phys. Rev. Lett. 67 (1991) 2818

85. M. Sosnowski, H. Usui, I. Yamada: Epitaxial growth of Cu films on Si by ionized cluster beam deposition; J. Vac. Sci. Technol. A 8 (1990) 1470

86. R. R. Chromik, W. K. Neils, E. J. Kotts: Thermodynamic and kinetic study of solid state reactions in the Cu-Si system; J. Appl. Phys. 86 (1999) 4273

87. R. A. Lukaszew, Y. Sheng, C. Uher, R. Clarke: Smoothening of Cu films grown on Si(001); Appl. Phys. Lett. 76 (2000) 724

88. L. Z. Mezey, J. Giber: The surface free energies of solid chemical elements: calculation from internal free enthalpies of atomization; Jpn. J. Appl. Phys. 21 (1982) 1569

89. B. D. Sartwell: Influence of ion beam activation on the mode of growth of $\mathrm{Cu}$ on Si(100); J. Vac. Sci. Technol. A 7 (1989) 2586

90. T. Michely, G. Comsa: Generation and nucleation of adatoms during ion bombardment of Pt(111); Phys. Rev. B 44 (1991) 8411

91. B. Degroote, A. Vantomme, H. Pattyn, K.Vanormelingen: Hyperthermal effects on nucleation and growth during low-energy ion deposition; Phys. Rev. B 65 (2002) 195401

92. G. Rosenfeld, R. Servatny, C. Teichert, B. Poelsema, G. Comsa: Layer-by-layer growth of Ag on Ag(111) induced by enhanced nucleation: A model study for surfactant-mediated growth; Phys. Rev. Lett. 71 (1993) 895

93. S. C. Wang, G. Ehrlich: Atom incorporation at surface clusters: An atomic view; Phys. Rev. Lett. 67 (1991) 2509 
94. R. Kunkel, B. Poelsema, L. K. Verheij, G. Comsa: Reentrant layer-by-layer growth during molecular-beam epitaxy of metal-on-metal substrates; Phys. Rev. Lett. 65 (1990) 733

95. M. Störmer, H.-U. Krebs: Structure of laser deposited metallic alloys; J. Appl. Phys. 78 (1995) 7080

96. K. H. Müller: Stress and microstructure of sputter-deposited thin films: Molecular dynamics investigations; J. Appl. Phys. 62 (1987) 1796

97. J. E. Yehoda, B. Yang, K. Vedam, R. Messier: Investigation of the void structure in amorphous germanium thin films as a function of low-energy ion bombardment; J. Vac. Sci. Technol. A 6 (1988) 1631

98. Y. H. Cheng, B. K. Tay, S. P. Lau, X. Shi, H. S. Tan: Influence of substrate bias on the microstructure and internal stress in $\mathrm{Cu}$ films deposited by filtered cathodic vacuum arc; J. Vac. Sci. Technol. A 19 (2001) 2102

99. C. Mottet, G. Tréglia, B. Legrand: Structures of a Ag monolayer deposited on $\mathrm{Cu}(111), \mathrm{Cu}(100)$ and $\mathrm{Cu}(110)$ substrates: An extended tight-binding quenchedmolecular-dynamics study; Phys. Rev. B 46 (1992) 16018

100. F. R. deBoer: Cohesion in metals: transition metal alloys; Amsterdam, NorthHolland, 1988

101. C. T. Horng, R. W. Vook: The initial epitaxy of Cu on (111) Ag films; J. Vac. Sci. Technol. 11 (1974) 140

102. F. K. LeGoues; M. Liehr; M. Renier: Epitaxy in the presence of very large misfit: high resolution TEM study of $\mathrm{Al} / \mathrm{Si}$, $\mathrm{Ag} / \mathrm{Si}, \mathrm{Al} / \mathrm{CaF}_{2} / \mathrm{Si}$ and $\mathrm{Ag} / \mathrm{CaF}_{2} / \mathrm{Si}$; Mat. Res. Soc. Proc. 315 (1987) 121

103. D. Winau, H. Itoh, A. K. Schmid, T. Ichinokawa: Ag on Si(001)(2×1) formation of a $2 \times 3$ superstructure; J. Vac. Sci. Technol. B 12 (1994) 2082

104. J. H. Je, T. S. Kang, D. Y. Noh: Epitaxial and island growth of Ag-Si(001) by rf magnetron sputtering; J. Appl. Phys. 81 (1997) 6716

105. T. C. Nason, L. You, T.-M. Lu: Room temperature epitaxial growth of Ag on low-index Si surfaces by a partially ionized beam; J. Appl. Phys 72 (1992) 466

106. A. Samsavar, E. S. Hirschorn, F. M. Leibsle, T.-C. Chiang: Scanning-tunnelingmicroscopy studies of Ag on Si(100)-(2 x 1); Phys. Rev. Lett. 63 (1989) 2830 
107. Park-K-H; Smith-G-A; Rajan-K; Wang-G-C: Interface characterization of epitaxial Ag films on Si(100) and Si(111) grown by molecular beam epitaxy; Metall. Trans. A 21A (1990) 2323

108. M. Kardar, G. Parisi, Y. C. Zhang: Dynamic Scaling of Growing Interfaces; Phys. Rev. Lett. 56 (1986) 889

109. P. Meakin, P. Ramanlal, L. M. Sander, R. C. Ball: Ballistic deposition on surfaces; Phys. Rev. A 34 (1986) 5091

110. Z.-W. Lai, S. Das Sarma: Kinetic growth with surface relaxation: Continuum versus atomistic models; Phys. Rev. Lett. 66 (1991) 2348

111. C. Thompson, G. Palasantzas, Y. P. Feng, S. K. Sinha, J. Krim: X-rayreflectivity study of the growth kinetics of vapor-deposited silver films; Phys. Rev. B 49 (1994) 4902

112. H. Li, D. Tian, J. Quinn, Y. S. Li, F. Jona: Low-energy electron diffraction and photoemission study of epitaxial films of $\mathrm{Cu}$ on Ag(001); Phys. Rev. B 43 (1991) 6342

113. D. T. Jiang, E. D. Crozier, B. Heinrich: Structure determination of metastable epitaxial $\mathrm{Cu}$ layers on $\mathrm{Ag}(001)$ by glancing-incidence $x$-ray-absorption fine structure; Phys. Rev. B 44 (1991) 6401

114. W. F. Egelhoff, I. Jacob: Reflection High-Energy Electron Diffraction (RHEED) Oscillations at 77 K; Phys. Rev. Lett. 62 (1989) 921

115. L. A. Bruce, H. Jaeger: Geometric factors in FCC and BCC metal-on-metal epitaxy. I. Deposits of Cu and Ni on (001) Ag; Philos. Mag. 36 (1977) 1331

116. A. L. Shull, F. Spaepen: Measurements of stress during vapor deposition of copper and silver thin films and multilayers; J. Appl. Phys. 80 (1996) 6243

117. W. F. Egelhoff, I. Jacob, J. M. Rudd, J. F. Cochran, B. Heinrich: X-ray photoelectron and Auger electron forward-scattering studies of lattice expansions and contractions in epitaxial films; J. Vac. Sci. Technol. A 8 (1990) 1582

118. T. Urano, T. Kanaji: Structures of iron films deposited on Si(111)7*7 surface studied by LEED; Appl. Surf. Sci. 33 (1988) 68

119. M. De Crescenzi, G. Gaggiotti, N. Motta, F. Patella, A. Balzarotti, J. Derrien: Bremsstrahlung-isochromat-spectroscopy and x-ray-photoelectron-spectroscopy 
investigation of the electronic structure of beta-FeSi2 and the Fe/Si(111) interface; Phys. Rev. B 42 (1990) 5871

120. H. Moritz, B. Rösen, S. Popovic, A. Rizzi, H. Lüth: Si(111)/FeSi2 heterostructures: Formation and properties of the low temperature metallic $(\gamma)$ and semiconducting ( $\beta$ ) disilicide phases; J. Vac. Sci. Technol. B 10 (1992) 1704

121. X. Wallart; H. S. Zeng; J. P. Nys; G. Dalmai: Electron spectroscopy study of the $\mathrm{Fe} / \mathrm{Si}(111)$ interface formation and reactivity upon annealing; Appl. Surf. Sci. 56 (1992) 427

122. Y. Ufuktepe; M. Onellion: Electronic structure of Fe overlayers on Si(111); Solid State Comm. 76 (1990) 191

123. J. M. Gallego, J. M. García, J. Alvarez, R. Miranda: Metallization-induced spontaneous silicide formation at room temperature: The Fe/Si case; Phys. Rev. B 46 (1992) 13339

124. B. Qi, R. M. Gilgenbach, Y. Y. Lau, M. D. Johnston, J. Lian, L. M. Wang, G. L. Doll, A. Lazarides: Ablation plasma ion implantation experiments: Measurement of Fe implantation into Si; Appl. Phys. Lett. 78 (2001) 3785

125. S. Wagner, E. Carpene, P. Schaaf, M. Weisheit: Formation of $\beta-\mathrm{FeSi}_{2}$ by excimer laser irradiation of ${ }^{57} \mathrm{Fe} /$ Si-bilayers; Appl. Surf. Sci. 186 (2002) 156

126. J. Alvarez, A. L. Vázquez de Parga, J. J. Hinarejos, J. de la Figuera, E. G. Michel, C. Ocal, R. Miranda: Initial stages of the growth of Fe on Si(111)7 × 7; Phys. Rev. B 47 (1993) 16048

127. John R. Smith and Amitava Banerjea: New Approach to Calculation of Total Energies of Solids with Defects: Surface-Energy Anisotropies; Phys. Rev. Lett. 59 (1987) 2451

128. A. R. Miedema, A. K. Niessen: The enthalpy of solution for solid binary alloys of two 4d-transition metals; CALPHAD: Computer Coupling of Phase Diagrams and Thermochemistry 7 (1983) 27

129. A. M. Mazzone: Molecular dynamics simulations of the intermixing of a metallic heterostructure irradiated at near-threshold energies; Nucl. Inst. Meth. Phys. Res. B 83 (1993) 495

130. F. Milstein, E. F. Huei, J. Marschall: Mechanics and energetics of the Bain transformation; Phil. Mag. A 70 (1994) 621 
131. H. Wawra: The surface energy of solid materials as measured by ultrasonic and conventional test methods; Z. Metallkunde 66 (1975) 492

132. W. F. Egelhoff: Structural Property Relationships for Metal/Metal Interfaces; MRS Symposia Proceedings 229 (1991) 27

133. S. Berg, I. V. Katardjiev: Preferential sputtering effects in thin film processing; J. Vac. Sci. Technol. A 17 (1999) 1916

134. G. P. Stern, J. C. Walker: Examination of epitaxial (111)Ag/(110)Fe heterostructures; J. Appl. Phys. 61 (1987) 4332

135. G. L. Krasko, G. B. Olson: Energetics of bcc-fcc lattice deformation in iron; Phys. Rev. B 40 (1989) 11536

136. S. P. Lau, Y. H. Cheng, J. R. Shia, P. Cao, B. K. Tay, X. Shi: Filtered cathodic vacuum arc deposition of thin film copper; Thin Solid Films 398 (2001) 539

137. X. Shi, B. K. Tay, D. I. Flynn, Q. Ye, Z. Sun: Characterization of filtered cathodic vacuum arc system; Surf. Coat. Technol. 94 (1997) 195

138. H. P. Bader, M. A. Lardon: Topographical limitations to the metallization of very large scale integrated structures by bias sputtering: Experiments and computer simulations; J. Vac. Sci. Technol. B 4 (1986) 1192

139. Y. G. Yang, X. W. Zhou, R. A. Johnson, and H. N. G. Wadley: Atomistic simulations of deep submicron interconnect metallization; J. Vac. Sci. Technol. B 20 (2002) 622 


\section{Danksagung}

Mein besonderer Dank gilt zunächst Prof. H.-U. Krebs, der diese Arbeit ermöglicht hat und stets mit viel Geduld und guten Ratschlägen $\mathrm{zu}$ ihrem Gelingen beigetragen hat. Neben Uli Krebs haben auch die anderen Mitglieder der Gruppe, Conni Mewes, Kai Sturm, Jörg Faupel, Erik Süske, Thorsten Scharf und Christian Fuhse durch ihre Hilfe im Labor, viele Diskussionen, wiederholtes Korrekturlesen halbfertiger Kapitel und ganz allgemein durch eine angenehme Arbeitsatmosphäre mit regelmäßigen Kafferunden sehr geholfen. Mit dem Weggang von Kai Sturm ist leider auch die Ära der Gummibärchen am Kaffeetisch vergangen...

Die ehemaligen Mitglieder Sebastian Fähler und Michael Störmer haben mir in der Anfangszeit der Arbeit sehr geholfen und versucht, ihr umfangreiches Wissen über die kleinen Tricks der Laborarbeit und die komplexen Vorgänge der Laserdeposition an mich weiterzugeben. Sebastians Lasersteuerungsprogramm leistet uns bis heute unersetzliche Dienste.

Ganz besonders möchte ich Prof. Satish Vitta danken, der mir und der gesamten Arbeitsgruppe die Kleinwinkel-Röntgenstreuung nahegebracht und damit eine sehr ergiebige Untersuchungsmethode erschlossen hat.

Für alles, was mit Mößbauerspektroskopie zu tun hat, habe ich Prof. Peter Schaaf und seiner Gruppe zu danken. Ohne sie wäre ein wichtiges Kapitel dieser Arbeit nicht entstanden. Die Diskussionen mit ihm und besonders auch mit Ratnesh Gupta haben mein Verständnis der Mößbauerspektroskopie sehr gefördert.

Den Mitgliedern der Werkstatt, insbesondere Bernd Droste, gilt mein Dank für die vielen exakt gefertigte Teile (wie z. B. das 28fach-Target-Substrat-Kombimagazin), die innerhalb und außerhalb der Vakuumkammer ihren Dienst tun. Auch mit passenden Schrauben, Blechen oder Eisengewichten wurde ich immer prompt versorgt.

Ich möchte nicht die vielen anderen Mitglieder des Institutes vergessen, die auf verschiedenste Weise ihren Beitrag geleistet und nicht zuletzt andere wichtige Qualifikationen gefördert haben, wie zum Beispiel die Hand-Auge-Koordination bei der konzentrierten Arbeit am Kickertisch. Euch allen vielen Dank! 


\section{Lebenslauf}

Geburtsdatum: 3. Juni 1973

Geburtsort: $\quad$ Erfurt

Eltern: $\quad$ Karlheinz und Adelheid Weisheit

Staatsangehörigkeit: Deutsch

September 1980 - Juli 1990

September 1990 - Juli 1992

26. Juni 1992

September 1992 - Juli 1994

8. Juli 1994

August 1994 - Juni 1995

Oktober 1995 - Oktober 1998

Oktober 1995 - September 1998

Juni 1997 - September 1998

29. Oktober 1998

Januar 1999 - heute
Polytechnische Oberschule 52 “Fritz Gäbler”, Erfurt

Institut zur Vorbereitung auf ein Auslandsstudium (IVA), Halle a. d. Saale

heutiger Name: Gymnasium am Reileck

Abitur

Grundstudium Physik, Universität Göttingen

Vordiplom Physik

Hauptstudium Physik, University of California at Berkeley

Haupstudium Physik, Universität Göttingen

Wissenschaftliche Hilfskraft als Systemadministrator, Geographisches Institut

Diplomarbeit am Institut für Metallphysik

Diplom Physik

Doktorarbeit am Institut für Materialphysik, Universität Göttingen 